This is the peer reviewed version of the following article: Chem. Eur. J. 2012, 18, 5655, which has been published in final form at http://onlinelibrary.wiley.com/doi/10.1002/chem.201200200/abstract. This article may be used for non-commercial purposes in accordance With Wiley-VCH Terms and Conditions for self-archiving

\title{
Ethynyl BenziodoXolones for the Direct Alkynylation of Heterocycles: Structural Requirement, Improved Procedure for Pyrroles and Insights into the Mechanism.
}

\author{
Jonathan P. Brand, Clara Chevalley, Rosario Scopelliti and Jérôme Waser*[a]
}

\author{
Dedication ((optional))
}

\begin{abstract}
This report describes a full study of the gold-catalyzed direct alkynylation of indoles, pyrroles and thiophenes using alkynyl hypervalent iodine reagents, especially the study of the structural requirements of alkynylbenziodoxolones for an efficient acetylene transfer to heterocycles. An improved procedure for the alkynylation of pyrroles using pyridine as additive is also reported. Nineteen alkynylbenziodoxol(on)es were synthesized and evaluated in the direct alkynylation of indoles and/or
\end{abstract}

thiophenes. Bulky silyl groups as acetylene substituents were optimal. Nevertheless, transfer of aromatic acetylenes to thiophene was achieved for the first time. An accelerating effect of a methyl substituent in both 3- and 6position of triisopropylsilylethynyl-1,2benziodoxol-3(1H)-one (TIPS-EBX) on the reaction rate was observed. Competitive experiments between substrates of different nucleophilicity, deuterium labelling experiments as well as the regioselectivity observed are all in agreement with electrophilic aromatic substitution. Gold(III) 2pyridinecarboxylate dichloride was also an efficient catalyst for the reaction. Investigations indicated that gold (III) could be eventually reduced to gold (I) during the process. As a result of these investigations, a $\pi$ activation or an oxidative mechanism are most probable for the alkynylation reaction.

Keywords: I $\bullet$ Alkynylation $\bullet$ Au $\bullet$
Heterocyclic compd. $\bullet$ Reactivity

\section{Introduction}

(Hetero)aryl acetylenes are widespread structures in organic synthesis, both as synthetic intermediates and targets. ${ }^{[1]}$ The unique reactivity of acetylenes allows a wide range of synthetic modifications via nucleophilic addition, cycloaddition, cycloisomerisation, reduction, oxidation and cross-coupling. In addition, heteroaryl acetylenes find application in material sciences. Both oligomeric ${ }^{[2]}$ and polymeric ${ }^{[3]}$ heteroaryl acetylenes can be used as molecular wires, liquid crystals, sensors and in many further important applications.

[a] J. P. Brand, C. Chevalley, Dr. R. Scopelliti, Dr. J. Waser Laboratory of Catalysis and Organic Synthesis, Institute of Chemica Sciences and Engineering Ecole Polytechnique Fédérale de Lausanne EPFL SB ISIC LCSO, BCH 4306, 1015 Lausanne, Switzerland. E-mail: jerome.waser@epfl.ch

Supporting information for this article is available on the WWW under http://www.chemeurj.org/ or from the author.
Two of the most often used methods for alkyne synthesis are triple bond formation from carbonyl compounds ${ }^{[4]}$ and acetylene transfer using cross coupling reactions such as the Sonogashira reaction. ${ }^{[5]}$ Despite the widespread use of these reactions, both need pre-functionalization of the heterocyclic ring, which could be challenging in complex structures when achieving high chemo- and regio-selectivity is difficult. Furthermore, the number of linear steps required to reach the target is also increased. As a result, more direct methods to access heteroaryl acetylenes via $\mathrm{C}-\mathrm{H}$ bond alkynylation would be highly desirable.

Numerous strategies for aryl-aryl bond formation directly from unactivated $\mathrm{C}-\mathrm{H}$ bonds have been developed ${ }^{\left[{ }^{[6]}\right.}$ In contrast, catalytic direct alkynylation methods were scarce up to 2009. [7] In 2002, Yamaguchi first reported the ortho alkynylation of phenols and anilines using $\mathrm{GaCl}_{3}$ and chloroacetylenes. ${ }^{[8]}$ In 2007, Gevorgyan developed the first palladium-catalyzed direct alkynylation of $\mathrm{N}$ heterocycles using bromoacetylenes. ${ }^{[9]}$ However, since 2009, the situation has radically changed ${ }^{[10]}$ and alkynylation of azoles, ${ }^{[11]}$ electron-rich aromatics ${ }^{[12,13]}$ and pentafluoroarenes ${ }^{[14,11 \mathrm{~d}]}$ have subsequently been reported. Nevertheless, there still exists a paucity of general methods which can be applied to a range of different classes of aryl substrates. Most of the strategies are based on the use of halogenoacetylenes (mostly bromoacetylenes). Additionally, there are a few examples of reactions using directly terminal acetylenes but these processes have usually a limited 
scope. ${ }^{[11 \mathrm{~d}, 12 c, 12 \mathrm{~d}, 14]}$ In order to develop more efficient and general methodologies, we decided to focus on more reactive alkynyl iodonium salts, which were studied intensively 20 to 30 years ago. ${ }^{[15]}$ Due to the excellent leaving group ability of PhI, alkynyl iodonium salts proved to be strongly electrophilic and reacted with keto esters, cuprates and heteroatom nucleophiles. However, alkynyliodonium salts were never used for the alkynylation of nucleophilic (hetero)aromatics.

In 2009, we reported the first example of the use of a hypervalent iodine reagent for the gold-catalyzed direct alkynylation of indoles and pyrroles (Scheme 1). ${ }^{[13 a]}$ Classical alkynyl iodonium salts derived from iodobenzene were not successful in this transformation, and we introduced triisopropylsilylethynyl-1,2benziodoxol-3(1H)-one (TIPS-EBX, 1) as an efficient acetylene transfer reagent. ${ }^{[16,17]}$ In the case of indoles, the process showed good to excellent yields, high $\mathrm{C} 3$ regioselectivity, a broad functional group tolerance and afforded easily deprotectable silyl acetylenes. For pyrroles, C2-alkynylated products were obtained in moderate to good yields. Importantly, this report was the first example of the combination of gold and hypervalent iodine for direct alkynylation. ${ }^{[18]}$

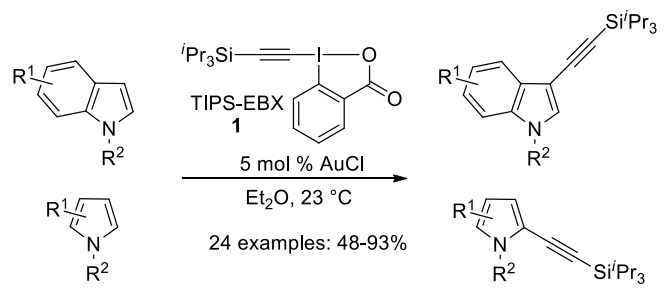

Scheme 1. Direct alkynylation of indoles and pyrroles.

Building upon this work, we later reported the first direct alkynylation of thiophenes using TIPS-EBX (1) (Scheme 2). ${ }^{[13 b]}$ Primary investigation using the conditions for indole alkynylation afforded only low yields. This is in accordance with the lower nucleophilicity of thiophene. Fine tuning of reaction conditions was not successful to improve the yields, but the discovery of a cooperative effect between the gold catalyst and a Brønsted acid (trifluoroacetic acid (TFA)) allowed the direct alkynylation of a broad range of thiophenes in $48-83 \%$ yield. The reaction was applied to a wide range of building blocks for material sciences.

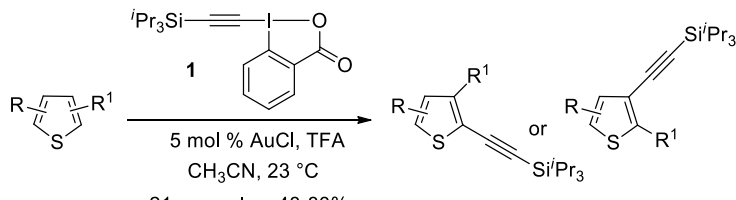

21 examples: $48-83 \%$

Scheme 2. Direct alkynylation of thiophenes.

Herein, we would like to report a further expansion of our work, including: (1) Improved conditions for the alkynylation of pyrroles, resulting in enhanced yields (from $48-79 \%$ to 56-97\%). (2) The first in depth studies of the influence of the reagent structure on reaction rate and efficiency. In the course of these studies, many unprecedented benziodoxolone reagents were synthesized and their structures studied by X-Rays. Several reagents with reactivity superior to TIPS-EBX were discovered. (3) Finally, further investigations toward the elucidation of the reaction mechanism are reported, including qualitative structure-reactivity relationships, kinetic isotope effects, intermediate trapping experiments, and studies on $\mathrm{Au}(\mathrm{III})$ vs $\mathrm{Au}$ (I) catalysis.

\section{Results and Discussion}

\section{Improved Procedure for Pyrrole Alkynylation}

In our previous work in the AuCl-catalyzed alkynylation of indoles and pyrroles, yields were usually lower for the latter (48-79\%). ${ }^{[13 a]}$ In particular, for applications in the functionalization of more complex valuable pyrroles, a more efficient protocol would be highly desirable. Pyrroles are very electron-rich heterocycles, and are sensitive to strong Lewis and Brønsted acids. We hypothesized that a competitive degradation of pyrroles by traces of $\mathrm{HCl}$ generated from $\mathrm{AuCl}$ during the reaction was at the origin of the lower yields observed. The mild base pyridine was consequently added to quench adventurous acid in the reaction mixture. In fact, the addition of 1.2 equivalent of pyridine led to a significant increase in yields (Table 1). The yield of the $\mathrm{C} 2$ alkynylation of $\mathrm{N}$ methylpyrrole (2a) was increased from $58 \%$ to $71 \%$ (entry 1). Interestingly, the $\mathrm{C} 2 / \mathrm{C} 3$ selectivity was increased from 1.9:1 to 4.2:1. The yield with 2-ethyl (2b) and 2-phenyl (2c) pyrrole were respectively enhanced from 58 and $60 \%$ to 81 and $84 \%$ (entries 2-3). Of note, the alkynylation of 2,4-dimethylpyrrole (2d) did not only afford $56 \%$ of monoalkynylated product but also $15 \%$ of bis alkynylation product (entry 4). In this case, $73 \%$ yield of bisalkynylated products was obtained using three equivalents of TIPS$\operatorname{EBX}(\mathbf{1})$.

Table 1. Improved alkynylation of pyrroles.

Entry

[a] Reaction conditions: $0.20 \mathrm{mmol}$ pyrrole $2,0.24 \mathrm{mmol}$ TIPS-EBX (1), $0.24 \mathrm{mmol}$ pyridine and $0.01 \mathrm{mmol} \mathrm{AuCl}$ in $4 \mathrm{~mL} \mathrm{Et}_{2} \mathrm{O}$ at $23{ }^{\circ} \mathrm{C}$ under air for $12-15 \mathrm{~h}$. Isolated yields after column chromatography. Yields without addition of pyridine are given in parenthesis. [b] Yields based on TIPS-EBX (1) with 3 equivalents of $N$-methylpyrrole (2a). [c] $15 \%$ of bis alkynylated product was isolated. $73 \%$ yield of bis-alkynylated product could be obtained when 3 equivalents of TIPS-EBX (1) were used. 
2,5-Disubstituted pyrroles were also efficiently alkynylated (entries 5-6). A nearly quantitative transformation was obtained with 1,2,5-trimethylpyrrole (2f) (Entry 6). In our previous work, trisubstituted pyrrole $\mathbf{2 g}$ was a particularly challenging substrate, and the desired product was obtained only in $48 \%$ yield, probably due to the three electron-donating substituents on the heterocycle. In the presence of pyridine, however, the alkynylation product was obtained in $83 \%$ yield (entry 7 ), which is very promising for the use of the method for especially challenging electron-rich pyrroles. Our original motivation for the use of pyridine was its basic properties. However, pyridine is also a potential ligand for gold. In order to distinguish between these two possible effects, we then examined ditertbutylpyridine as additive. To our surprise, no increase in yield was observed. This result might indicate that pyridine is acting as a ligand and not as a base, and the lower yields obtained in the absence of pyridine was due to a higher Lewis acidity of the gold catalyst.

\section{Synthesis and Study of Alkynyl Benziodoxolones.}

Essential for the success of the alkynylation reaction was the replacement of established alkynyliodonium salts by EBX-type reagents. In fact, in presence of Au catalysts, alkynyliodonium salts only yielded diyne products, and the desired alkynylation was not observed. When considering the key role of the reagent structure, we decided to realize a more precise structure-reactivity relationship study of the alkynyl benziodoxolone reagents. One important goal for these studies was to further understand the unique reactivity of alkynylbenziodoxolones in the quest of even more efficient reagents We were also motivated by the recent report of Fujii and Ohno on the favorable effect of a nitro group para to the iodine in $\mathrm{a} \mathrm{Cu}$ catalyzed annulation reaction, ${ }^{[19]}$ and wanted to see if such an effect would also be observed in Au-catalyzed reactions. A second important objective of this work was to examine if the method could also be extended to the transfer of alkynes without a silyl protecting group. In this context, the goal was to examine the potential of the reagents in a broad sense, and not yet to develop truly efficient processes.

Alkynylbenziodoxolones were first reported and structurally characterized by Ochiai in 1991. ${ }^{[20]}$ In 1996, Zhdankin published an improved synthesis of this class of reagents and also synthesized for the first time silyl alkynylbenziodoxolones, including TIPS-EBX (1). ${ }^{[17]}$ Nevertheless, these compounds did not find application in organic synthesis despite the utility of the parent alkynyliodonium salts. We optimized the synthesis of TIPS-EBX (1) to afford the reagent in $86 \%$ yield on a $30 \mathrm{~g}$ scale without column chromatography from iodosylbenzoic acid (4) (Equation 1). On a large scale, acidic work-up to remove pyridine followed by a basic work-up to remove iodobenzoic acid were crucial to obtain reproducible yield and purity.

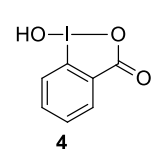

a) $\mathrm{Me}_{3} \mathrm{SiOTf}, \mathrm{CH}_{3} \mathrm{CN}$

b) $\mathrm{Me}_{3} \mathrm{Si}=\mathrm{Si}^{i} \mathrm{Pr}_{3}$

c) pyridine

d) Recrystallization $\left(\mathrm{CH}_{3} \mathrm{CN}\right)$

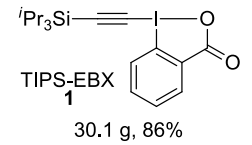

For both indoles and thiophenes, TIPS-EBX (1) had proven a superior alkynylation reagent over TMS-EBX (5a). In order to better quantify the importance of steric bulk on the silicon atom, further silylated EBX reagents of increasing size were synthesized (Scheme 3). A first difference was already apparent during the preparation of the reagents: the Zhdankin protocol worked well for sterically hindered silyl groups, but a problem with yield reproducibility was observed for smaller protecting groups, especially TMS. We then discovered that partial decomposition of the reagent often occurred upon treatment with pyridine. A milder neutralization using sodium bicarbonate led to a more reproducible synthesis of the desired reagents.

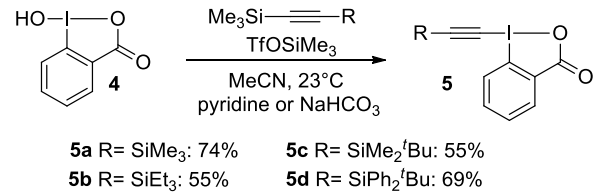

Scheme 3. Synthesis of silylethynylbenziodoxolones.

All new reagents were first tested in the alkynylation of indole (6) (Scheme 4). Reagents with bulky silyl groups such as $\mathrm{SiMe}_{2}{ }^{t} \mathrm{Bu}$ (5c) and $\mathrm{SiPh}_{2}{ }^{t} \mathrm{Bu}$ (5d) gave similar results as TIPS-EBX (1). However, both TMS- (5a) and $\mathrm{SiEt}_{3}(\mathbf{5 b})$ EBX gave poor results in $\mathrm{Et}_{2} \mathrm{O}$ (as well as low reproducibility for TMS-EBX (5a)). Due to their low solubility, the reaction was carried out in $\mathrm{CH}_{3} \mathrm{CN}$. TMSEBX (5a) still did not afford any product, whereas the $\mathrm{SiEt}_{3}$ reagent $\mathbf{5 b}$ afforded $57 \%$ of the alkynylated product.

In order to investigate the reason for the low yield obtained for small groups, we investigated the stability of the catalyst in the presence of TMS-EBX (5a). When TIPS-EBX (1) and indole (6) were added to a premixed solution of $\mathrm{AuCl}$ and TMS-EBX (5a), a low yield was obtained $(8 \%)$. On the contrary, premixing of TIPSEBX (1) and $\mathrm{AuCl}$ did not lead to a decrease of yield. This result may indicate the degradation of $\mathrm{AuCl}$ by TMS-EBX (5a).

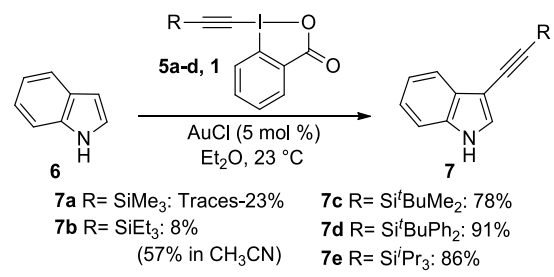

Scheme 4. Alkynylation of indole (6) using silylethynylbenziodoxolones.

When TMS-EBX (5a) was used for the alkynylation of 2hexylthiophene $(\mathbf{8})$, only traces of product were observed (Scheme 5). In contrast to what had been observed for indole alkynylation, no TMS-EBX (5a) was remaining. This result is probably due to the degradation of the reagent under acidic conditions. A steady improvement of the yield was observed by increasing the size of the silyl group ( $\mathrm{SiEt}_{3}, \mathrm{SiMe}_{2}{ }^{t} \mathrm{Bu}$ and $\mathrm{SiPh}_{2}{ }^{t} \mathrm{Bu}$ ). Importantly, whereas the triisopropylsilyl product was difficult to separate from the starting material, the tertbutyldiphenylsilyl group allowed an easier separation.

The high efficiency observed for the transfer of silyl acetylenes is important for practical applications, as easy deprotection of the products gave access to terminal acetylenes, which can then be further functionalized. Nevertheless, the transfer of aryl and alkyl acetylenes directly would allow a more convergent synthesis of complex compounds. For this reason, we decided to investigate acetylene transfer using aryl and alkyl EBX reagents. 


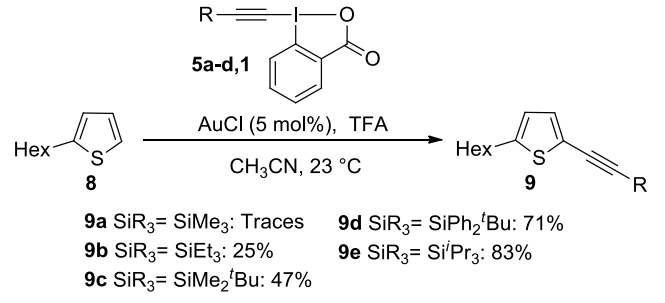

Scheme 5. Alkynylation of 2-hexylthiophene (14) using silylethynylbenziodoxolones. Hex $=$ Hexyl.

Again, the use of $\mathrm{NaHCO}_{3}$ instead of pyridine for neutralization and benziodoxole ring closure led to a reproducible synthesis of $\mathrm{Ph}$ EBX (5e) in $46 \%$ yield. In order to examine steric and electronic effects on the aryl acetylene, the synthesis of mesitylene, paranitrobenzene and para-methoxybenzene reagents was then attempted. None of these reagents have been previously reported. Mesitylene (5f) and para-nitrophenyl EBX (5g) were obtained in moderate yields (respectively 30 and 59\%). Unfortunately, no product was obtained for para-methoxybenzene due to the fast degradation of the product under the reaction conditions. In addition to aromatic groups, alkyl acetylenes were also investigated. Gratifyingly, the conditions developed for Ph-EBX (5e) proved to be efficient for the very sensitive ${ }^{n} \mathrm{Bu}-\mathrm{EBX}$ (5h) (28\% yield). In contrast to other reagents, this product was not purified by recristallization but by flash chromatography. ${ }^{t} \mathrm{Bu}-\mathrm{EBX}(\mathbf{5 i})$ was synthetized using the procedure reported by Zhdankin. ${ }^{[17]}$

When Ph- (5e) and ${ }^{n} \mathrm{Bu}-(\mathbf{5 h})$ EBX were submitted to the reaction conditions with indole (6), only traces of product were obtained (Scheme 6). In the case of ${ }^{t} \mathrm{Bu}-\mathrm{EBX}$, the alkynylated product was obtained in $25 \%$. Although this yield is still low, it constituted the first example of transfer of an alkyl acetylene using gold catalysis.
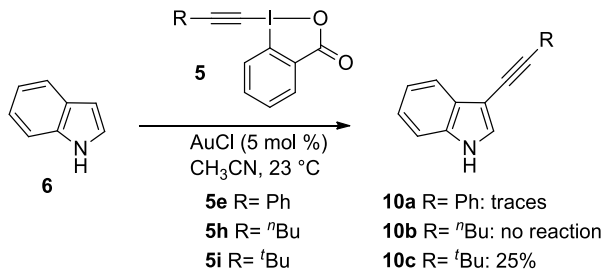

Scheme 6. Alkynylation of indoles using phenyl and alkyl ethynylbenziodoxolones.

In the case of 2-hexyl-thiophene (8), aromatic EBX reagents afforded products in moderate yields (Table 2, entries 1-3). These results are promising, and future work will be directed towards the optimization of this highly convergent synthesis of arylated alkynyl thiophenes. Alternatively, arylacetylenes can also be obtained in higher yields from the TIPS-acetylene products in a one-pot deprotection-Sonogashira sequence. ${ }^{[21]}{ }^{n} \mathrm{Bu}-\mathrm{EBX} \quad(\mathbf{5 h})$ was unsuccessful for the alkynylation of thiophene (entry 4), but ${ }^{t} \mathrm{Bu}$ EBX (5i) gave $36 \%$ yield (entry 5 ).

The difference in reactivity of aromatic alkynyl benziodoxolones between indoles and thiophenes was intriguing, as generally indoles were the most reactive substrates in our methodology. We wondered if the relative success obtained with 2hexylthiophene (8) was due to the presence of TFA. In fact, when indole $(\mathbf{6})$ was submitted to thiophene alkynylation conditions with
Ph-EBX (5e), a mixture of C2 and C3 alkynylated indoles 12 was observed by ${ }^{1} \mathrm{H}$ NMR in only 5 minutes (Scheme 7). Unfortunately, significant batch to batch variations in both yield (33 to 47\%) and regioselectivity (C2/C3 60 to $100 \%)$ were observed.

Table 2. Alkynylation of 2-hexylthiophene (8) using phenyl and alkyl ethynylbenziodoxolones.

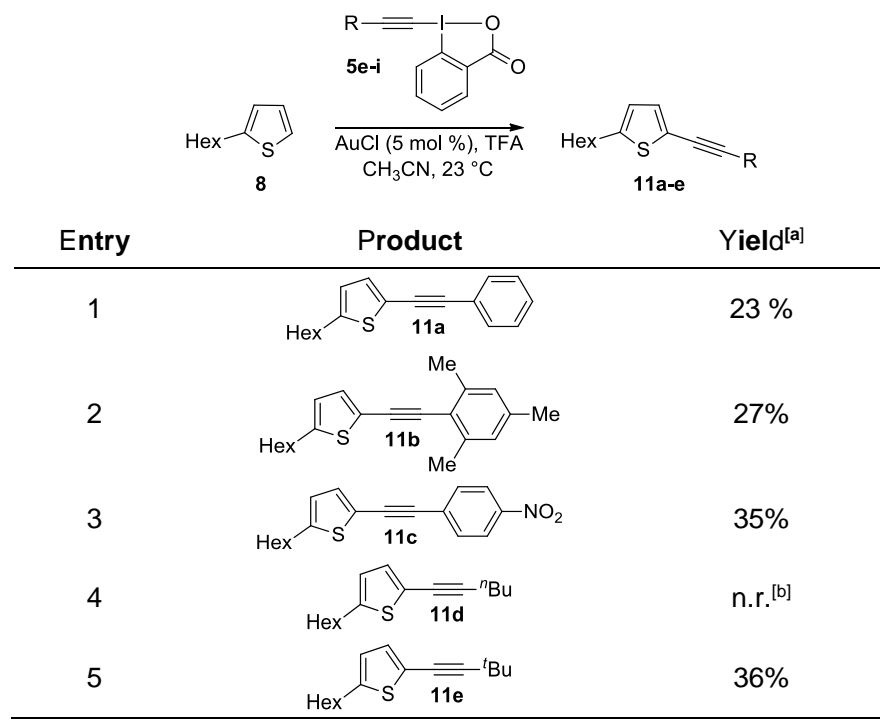

[a] Reaction conditions: $0.40 \mathrm{mmol} \mathbf{8}, 0.48 \mathrm{mmol}$ benziodoxolone, $5 \mathrm{~mol} \% \mathrm{AuCl}, 0.48$ mmol TFA, $0.2 \mathrm{M}, \mathrm{rt}, 14-36 \mathrm{~h}$. Isolated yield after column chromatography. [b] n.r.: no reaction. Hex $=$ Hexyl.

In order to investigate whether this lower regioselectivity was due to the reaction conditions or the structure of the reagent, we used TIPS-EBX (1) with indole (6) under the same conditions. In this case however, substitution at position 3 only was obtained in $29 \%$ yield. The lower yields obtained with indole (6) in presence of acids can be rationalized by the higher acid-sensitivity of this electron-rich heterocycle. The change of regioselectivity with $\mathrm{Ph}$ EBX (5e) is more intriguing, and perhaps indicates a change of mechanism depending on the reagent and the addition of acid.

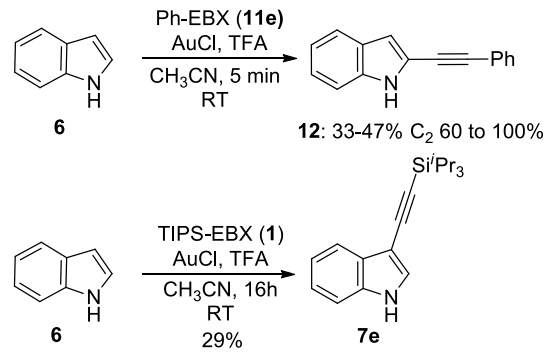

Scheme 7. Alkynylation of indole (6) using ethynylbenziodoxolones and TFA.

This investigation on the variation of the alkynyl substituent clearly showed the superiority of bulky silyl groups. It can be hypothesized that the improved solubility and stability of bulky silyl EBX reagents can explain their higher efficiency in the alkynylation of heterocycles. Furthermore, the more electron-rich C-Si bonds could also play a role to explain the exceptional reactivity of these reagents. Preliminary results were also obtained for the transfer of aryl acetylenes in the case of 2-hexylthiophene (8), but further improvement is required in this case. 
The influence of substituents on the benziodoxolone aromatic ring was then investigated in order to increase the reactivity of TIPS-EBX (1). A range of EBX reagents bearing electron-donating or electron-withdrawing groups as well as bistrifluoromethylsubstituted benziodoxole $\mathbf{1 5} \mathrm{g}$ were synthesized in moderate to good yields using Zhdankin procedure (Scheme 8). ${ }^{[17]}$ In addition, a protonated benziodoxolone $\mathbf{1 5 h}$ was also synthesized using a known method. ${ }^{[17]}$
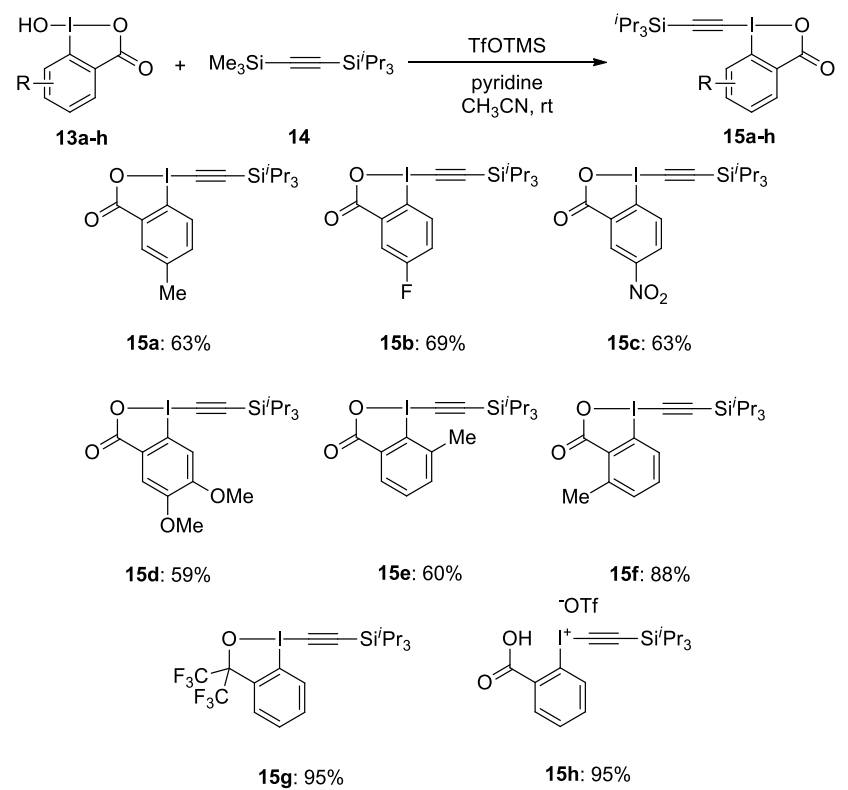

Scheme 8. Synthesis of analogues of TIPS-EBX (1).

Togni reported the efficiency of dimethylbenziodoxole structure in $\mathrm{CF}_{3}$ transfer. ${ }^{[22]}$ In contrast to bistrifluoromethyl-substituted benziodoxole, alkynyl dimethylbenziodoxole $\mathbf{1 7}$ has never been synthesized. Unfortunately, no product was obtained when Zhdankin's method was used. However, we discovered that the addition of lithiated triisopropylsilylacetylene to benziodoxole $\mathbf{1 6}$ activated by TMSOTf led to $\mathbf{1 7}$ in $86 \%$ of yield (Equation 2). $\mathbf{1 7}$ represents a highly interesting new electrophilic acetylene due to the higher trans effect present in dimethylbenziodoxole. ${ }^{[23]}$
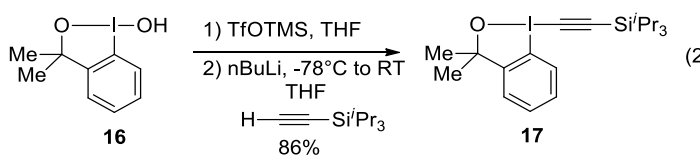

Under standard reaction conditions with indole (6), all substituted benziodoxolones gave the product in yields comparable to TIPS-EBX (1) (86\%, entries 1-7, Table 3). Interestingly, bis $\mathrm{CF}_{3}$ benziodoxole $\mathbf{1 5 g}$ afforded a mixture of the $\mathrm{C}_{3}$ alkynylated (43\%) and $\mathrm{C}_{2}$ alkynylated (15\%) products (entry 8). Protonated benziodoxolone $15 \mathrm{~h}$ did not form any product and degradation of indole (6) was observed (entry 9). Dimethylbenziodoxole 17 only afforded traces of product (entry 10). This result showed the highly different properties of $\mathbf{1}, \mathbf{1 5} \mathrm{g}$ and $\mathbf{1 7}$. The reaction was consequently only minimally influenced by substitution on the benzene ring, but the carbonyl group was an essential component of the reagent for an efficient alkynylation.
Table 3. Alkynylation of indole (6) using analogues of TIPS-EBX (1).

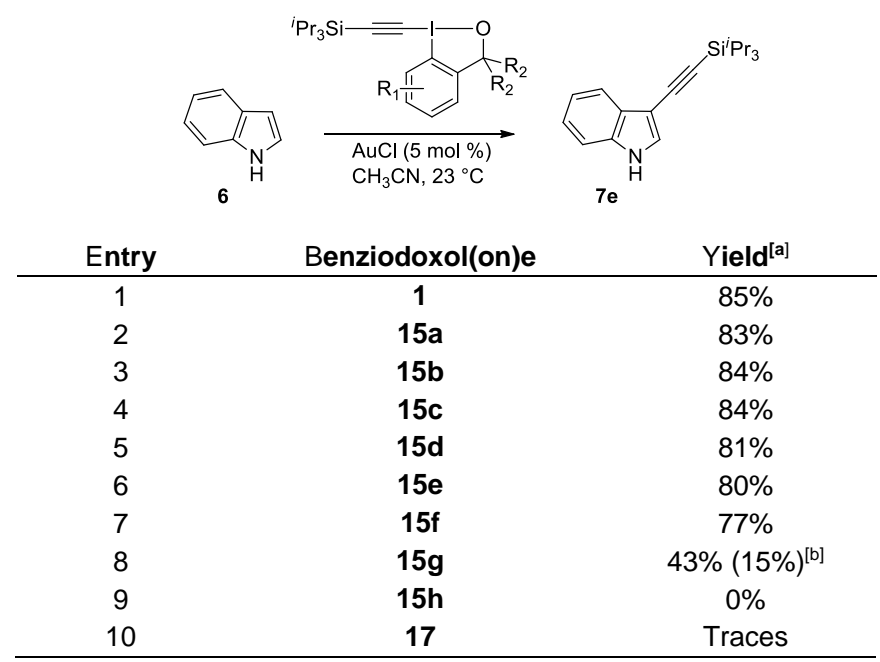

[a]Reaction conditions: $0.10 \mathrm{mmol}$ indole (6), $0.12 \mathrm{mmol}$ benziodoxol(on)e, and 0.01 $\mathrm{mmol} \mathrm{AuCl}$ in a $0.025 \mathrm{M}$ solution of undecylcyanide in $\mathrm{CH}_{3} \mathrm{CN}(2 \mathrm{~mL})$ at $23{ }^{\circ} \mathrm{C}$ under air for $14 \mathrm{~h}$. GC/MS yield using undecylcyanide as internal reference. [b] $\mathrm{C}_{2}$ alkynylation product.

\section{Mechanistic Investigations}

The reactivity and properties of both gold and hypervalent iodine have recently attracted broad interest. Gold complexes have been first established as excellent catalysts for the activation of $\pi$ systems. ${ }^{[24]}$ More recently, other types of reactions involving changes in the oxidation state of the gold catalyst have also incited a strong interest in the scientific community. ${ }^{[25]}$ Conversely, hypervalent iodine reagents are involved in oxidative and atom transfer processes, which have been proposed to proceed either via two electrons or SET mechanisms. ${ }^{[26,27]}$ Based only on these results in the literature, several pathways are possible for the Au-catalyzed alkynylation reaction. The three main mechanisms envisaged are: $\pi$ activation mechanism (Scheme 9), $\mathrm{Au}(\mathrm{I}) / \mathrm{Au}(\mathrm{III})$ mechanism (Scheme 10) and Lewis acid activation of the benziodoxolone, which can be followed either by a SET mechanism or a direct attack on the iodine (Scheme 11).

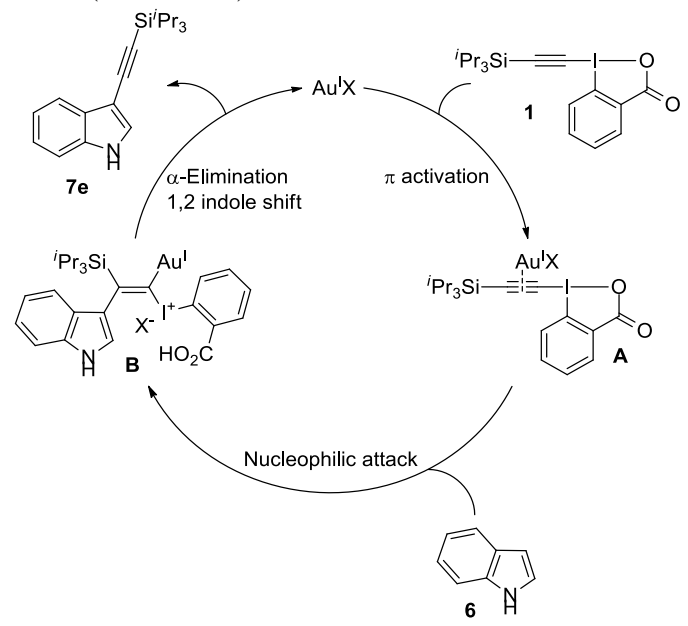

Scheme 9. $\pi$ Activation mechanism.

The first step of the $\pi$ activation mechanism involves a coordination of the triple bond by gold chloride that leads to an 
increased electrophilicity (Scheme 9). ${ }^{[24]}$ A Friedel-Craft type reaction of indole (6) at the most electrophilic $\beta$ position of the alkynylbenziodoxolone would then be in accordance with the inherent reactivity of alkynyl iodoniums. ${ }^{[28]}$ This step is expected to follow an electrophilic aromatic substitution mechanism. The vinyl gold intermediate $\mathbf{B}$ can then undergo an $\alpha$ elimination to generate a carbene, which then rearranges to form the triple bond (FritschButtenberg-Wiechell-type rearrangement).

An alternative mechanism involves an oxidative addition of gold in the $\mathrm{C}$-I bond to form the $\mathrm{Au}$ (III) intermediate A (Scheme 10). [25,29] The highly electrophilic gold(III) species can then undergo indole auration via electrophilic aromatic substitution. A reductive elimination then affords alkynylated indole $\mathbf{7 e}$.

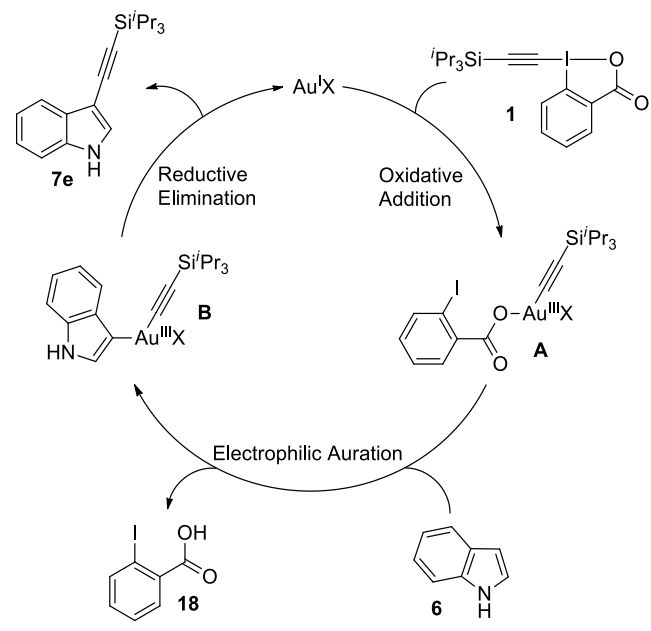

Scheme 10. Oxidative mechanism.

$\mathrm{AuCl}$ could also act as a Lewis acid and increase the electrophilicity of the iodine atom (Scheme 11). ${ }^{[22]}$ The activated hypervalent iodine $\mathbf{A}$ can then form a charge transfer complex $\mathbf{B}$ with the electron-rich heterocycle. According to Kita, ${ }^{[26 c]}$ a single electron transfer can occur to form C. C can then either directly rearrange to 7e via alkyne transfer (path a) or form the iodine(III) intermediate $\mathbf{D}$, which then gives $\mathbf{7 e}$ via a subsequent ligand coupling (path b). A direct nucleophilic attack of indole (6) on A can also generate $\mathbf{D}$ via a two electron transfer (path c).

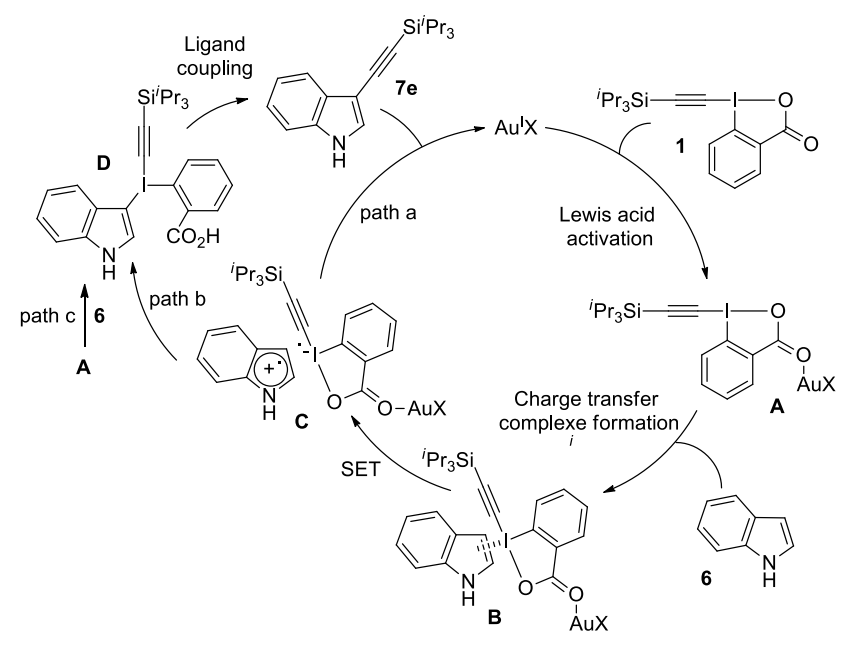

Scheme 11. Lewis acid activation mechanism
In the three mechanisms, an attack of unactivated indole (6) as nucleophile has been proposed. Another possible alternative would be nucleophilic activation of indole (6) by auration on the three position. ${ }^{[30]}$ The formed Au-complex would then act as nucleophile instead of indole (6) in the described catalytic cycles.

Unfortunately, the study of the mechanism of the gold-catalyzed alkynylation with TIPS-EBX (1) is made more difficult by the characteristics of the reaction. First, reproducible kinetics measurements are very difficult due to the heterogenous nature of gold chloride. Second, electron-rich ligands like phosphines or carbenes inhibit the reaction, even if the more reactive cationic gold complexes are used. In our previous communication, we used only simple triphenylphosphine cationic complexes, but when the screen was extended to other ligands recently introduced in gold catalysis, no better results were obtained. ${ }^{[31]}$ This is a major drawback for mechanistic investigations, as well-defined metal complexes would potentially allow the characterization of reactive intermediates, which is particularly challenging for gold species. In fact, vinyl gold species could be recently isolated, but this was only possible using $\mathrm{N}$-heterocyclic carbene ligands or phosphines. ${ }^{[32]}$ Furthermore, phosphine NMR is a valuable tool for studying the structure and oxidation state of metal complexes. Consequently, mostly indirect evidence had to be used to better understand the reaction.

Prior to describing new experiments, it is important here to briefly summarize the knowledge gathered through our previous work on the alkynylation of indoles and thiophenes: ${ }^{[13 a-b]}$

- The alkynylation method showed a regioselectivity consistent with an electrophilic aromatic substitution (C3 of indole, $\mathrm{C} 2$ of pyrrole and thiophene).

- $\mathrm{AuCl}$ was always the best catalyst, but with indole moderate yields could also be obtained with $\mathrm{AuCl}_{3} .{ }^{[33]}$

- An isolated gold thiophene complex did not react with TIPSEBX (1). Protonated benziodoxole $\mathbf{1 5 h}$ was also not able to transfer an acetylene to 2-hexylthiophene (8).

- Reaction with a ${ }^{13} \mathrm{C}$-labeled EBX reagent 19 showed that no silicium shift occurred during the reaction (Equation 3). Ochiai reported that the addition of $\alpha$-ketoester nucleophile on alkynyliodonium proceeds via carbene formation followed by $1,2-$ shift of the best migrating group, ${ }^{[28]}$ often silicium or hydrogen. As a result, addition of indole (6) in $\alpha$ position followed by TIPS 1,2 shift can be ruled out. On the other hand, 1,2-shift of indole cannot be excluded as aromatic group are known to be prone to this type of rearrangement.

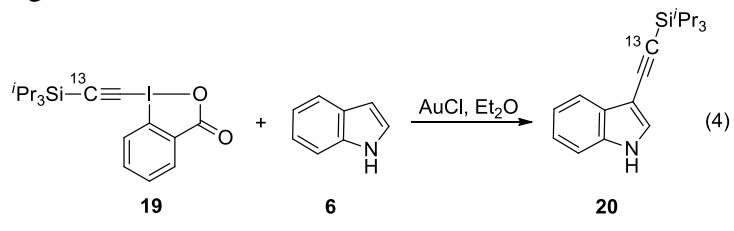

The regioselectivity results obtained are in agreement with what would be expected for an electrophilic aromatic substitution. Nevertheless in this study we discovered that the regioselectivity for the alkynylation of indole was dependent on the structure of the reagent (benziodoxole vs benziodoxolone), which seemed to indicate that several mechanisms could be possible. On the other hand, substitution of the benzene ring of the reagents showed surprisingly little effect on the yield. 
In this section, we will first present further experiments which indicated that a Lewis Acid or a SET mechanism was less probable. In order to further investigate the mechanism, we decided then to concentrate our efforts on semi-quantitative competitive experiments to gain a better insight into the kinetics of the reaction. Furthermore, the fact that $\mathrm{Au}(\mathrm{III})$ was also a catalyst for the reaction was highly interesting, as it could be better rationalized by a $\pi$ activation mechanism: we consequently decided to investigate $\mathrm{Au}(\mathrm{III})$ catalysts in more detail.

\section{SET and Lewis Acid Mechanisms}

Single electron transfer pathways are less likely for two reasons. First, the high electrophilic aromatic substitution regioselectivity observed with indoles would not be in agreement with SET processes, for which C2 substitution would be expected. ${ }^{[26 c]}$ The case is less clear for thiophenes, as in this case both electrophilic aromatic substitutions and SET processes give $\mathrm{C} 2$ functionalization. Second, no formation of heterocyclic dimers was observed, which is a frequent process for heterocyclic radical cations. ${ }^{[26 c]}$ Furthermore, the reaction was also done in the presence of one equivalent of $\mathrm{TMSN}_{3}$ which has been demonstrated to react rapidly with indole or thiophene radical cations. ${ }^{[26 \mathrm{~b}]}$ For both indole (6) and 2hexylthiophene (8), no addition of azide on the heterocycle was observed. However, it is difficult to exclude the presence of a tight radical pair, which would react too rapidly to be trapped. Direct attack on iodine, Lewis acid-catalyzed or not, also appeared less probable for us, although it is often proposed for trifluoromethylation using benziodoxolone reagents. ${ }^{[22]}$ In fact, no alkynylation was observed for indole (6) and/or 2-hexylthiophene (8) in an extended screening of Lewis and Brønsted acids including $\mathrm{HCl}$, TsOH, TFA, $\mathrm{FeCl}_{3}, \mathrm{AlCl}_{3}, \mathrm{Zn}(\mathrm{OTf})_{2}, \mathrm{Yb}(\mathrm{OTf})_{3}$ and $\mathrm{In}(\mathrm{OTf})_{3}$. The unique reactivity of $\mathrm{AuCl}$ would be very difficult to explain, as simple Lewis and Brønsted acids should be even more efficient to promote the reaction. In addition, control experiments at high temperature without the gold catalyst did not afford any product although this has been observed for arylation reactions using iodonium salts. ${ }^{[34]} \mathrm{A}$ first important conclusion of these mechanistic studies is consequently that an activation of the I-O bond followed by reaction on iodine is not probable. Consequently, the Aucatalyzed alkynylation reaction seems to be mechanistically distinct from other reactions using benziodoxolone reagents, in particular trifluoromethylation.

\section{Competitive Experiments}

As complete kinetic studies were difficult because of the heterogenous nature of $\mathrm{AuCl}$, we then turned to competitive experiments to gain a semi-quantitative insight into the reaction rate. The alkynylation was carried out with mixtures of indole (6) $(\mathrm{N}=5.55), 5$-cyanoindole $(\mathbf{2 1})(\mathrm{N}=2.83)$ and 5-methoxyindole $(\mathbf{2 3})$ ( $\mathrm{N}=6.22)$, as the nucleophilicity of these substrates has been described quantitatively (Scheme 12). ${ }^{[35]}$ The reactivity pattern observed is correlated with the nucleophilicity according to the Mayr's scale, as the ratio of products was 3.0:1 between indole (6) and 5-cyanoindole (21), and 3.7:1 between 5-methoxyindole (23) and 5-cyanoindole (21) respectively. Although the differences of reactivity are lower than in Mayr's model reaction for the reaction of nucleophile with an electrophile, this result certainly confirms that an electrophilic attack on the indole is part of the rate determining step of the reaction. This result is in agreement with the high regioselectivity observed for the most electron-rich position of the functionalized heterocycles.
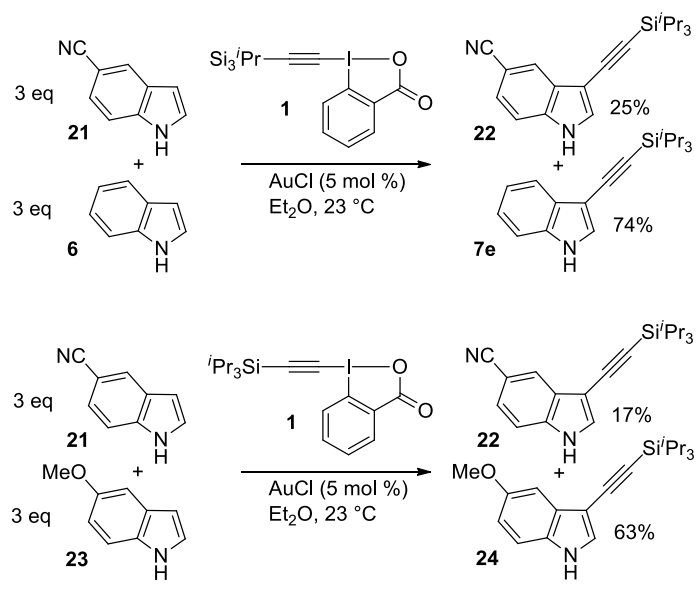

Scheme 12. Competitive experiments with indoles.

A qualitative determination of a potential kinetic isotope effect was then achieved by reaction of $37 \%$ deuterated indole 25 with 0.6 equivalents of TIPS-EBX (1) (Equation 4). When 25 was submitted to the reaction conditions, a slight enrichment in deuterium was observed. However, performing the reaction in the absence of TIPSEBX (1) showed a significant loss of deuterium even without reaction, making interpretation of this result impossible (not shown). We hypothesized that this outcome could be due to traces of acid or reversible auration of indole 25. Based on our previous results, we repeated these reactions in the presence of pyridine. In this case, the same deuterium enrichment was observed both with and without TIPS-EBX (1). Although the reason for this effect is not clear, the same result obtained in both cases demonstrated that there is no significant kinetic deuterium effect in the alkynylation reaction itself Consequently, it appears that cleavage of the C-H bond is not occurring during the rate-limiting step. This would be in agreement with a rate-limiting electrophilic attack on the indole, followed by a fast proton transfer and re-aromatization. In the case of thiophene, as it is not possible to slow down proton-deuterium exchange by the addition of pyridine, no conclusive results could be obtained.

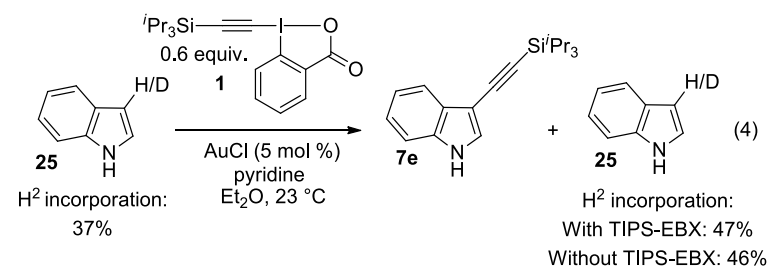

\section{$\mathrm{Au}(\mathrm{III})$ Catalysis}

At this point, we decided to re-investigate $\mathrm{Au}(\mathrm{III})$ catalysts for the reaction, as we hoped it could help us exclude a mechanism involving redox catalysis. In particular, we found that gold 2pyridinecarboxylate dichloride (26) was a good catalyst for the alkynylation of indole (6) (Equation 5). Furthermore, this catalyst is much better defined than $\mathrm{AuCl}$ and clear solutions were obtained during the reaction. Despite the fact that we cannot be sure that both reactions have the same mechanism, this cleaner reaction profile motivated us to compare the reactivity of the different synthesized alkynyl benziodoxolones (Figure 1). In particular, substitution on the benzyl ring of the EBX reagents had led to no changes in yield. By studying the full profile of the reaction instead of just the yield, we hoped to be able to detect subtle effects that we had missed in the preparative reactions. 


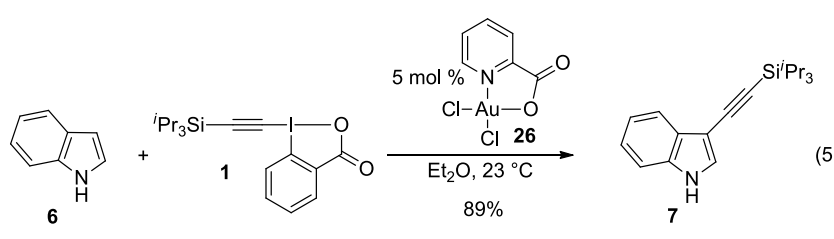

5-MethylTIPS-EBX (15a) and 4,5-dimethoxyTIPS-EBX (15d) gave similar kinetics as TIPS-EBX (1). ${ }^{[36]}$ In contrast, 5-fluoro (15b) and 5-nitroTIPS-EBX (15c) have higher initial rates, even if they gave slightly lower final yields (Figure 1). Interestingly, 3methylTIPS-EBX (15e) and 6-methylTIPS-EBX (15f) led to the highest reaction rates.
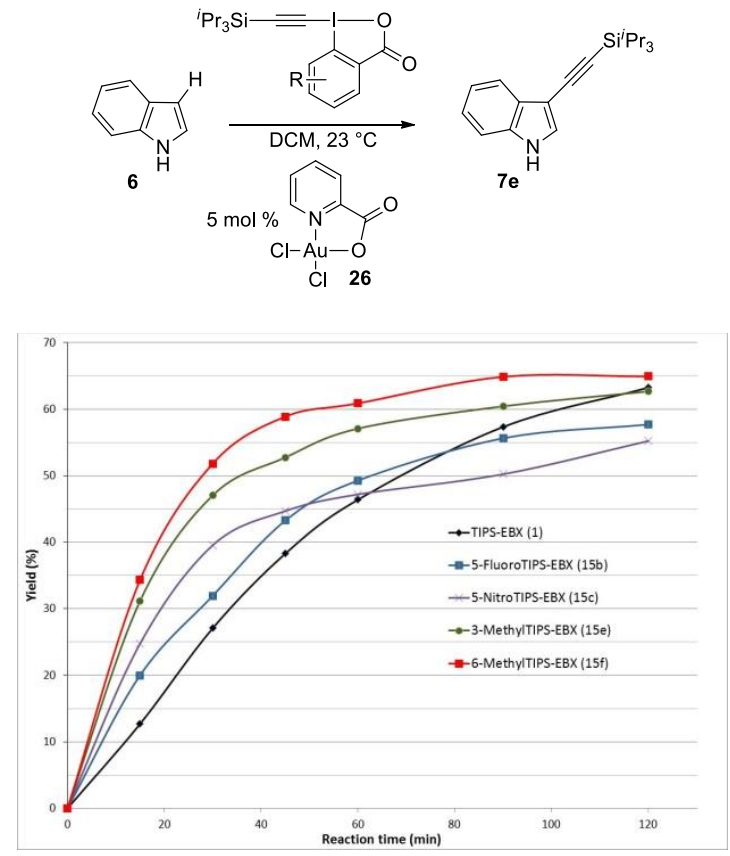

Figure 1. Kinetic profile for the alkynylation of indole (6) using triisopropy ethynylbenziodoxolones.

The observed kinetic is difficult to explain: an accelerating effect of the para electron-withdrawing group would be in accordance with a rate-limiting step involving electrophilic attack of the reagent ( $\pi$ activation mechanism, Scheme 9). However, the effect is weak, and could eventually also be explained by a ligand effect during the auration step in the oxidative mechanism (Scheme 10). The even stronger effect observed by the introduction of a methyl group in 3 or 6 positions is startling. In order to better understand the reactivity, we decided to analyze the structure of the reagents by X-ray. First, high-quality crystals of TIPS-EBX (1) were obtained by recrystalization from $\mathrm{CH}_{3} \mathrm{CN}$. In accordance with previously published X-ray structures of alkynylbenziodoxolones, ${ }^{[17,20]}$ the T-shape of the hypervalent iodine was confirmed. Furthermore, the alkyne is nearly in the same plane as the benziodoxolone (torsion angle C8-I1-C7-C6: 5. $8^{\circ}$ ) (Figure 2).

The structure of TIPS-EBX (1) was then compared with the two methyl-substituted reagents $\mathbf{1 5 e}$ and $\mathbf{1 5 f}$. In the case of 3methylTIPS-EBX (15e), the steric bulk of the methyl group forces the alkyne substituent outside the plane of the ring (torsion angle C8-I1-C7-C6: $34^{\circ}$ ) (Figure 3). In this case, a weaker 3-centers-4electrons hypervalent bond can be expected, and consequently a more reactive reagent. ${ }^{[37]}$ 6-methylTIPSEBX (15f), however, displayed a nearly perfectly planar structure (torsion angle C8-I1-
C7-C6: $0.7^{\circ}$ ) (Figure 4). In this case, the accelerating effect is more difficult to rationalize. Nevertheless, it is interesting to observe that the I1-O1 bond is shorter than in TIPS-EBX (1) (2.308 vs $2.336 \AA$ ) and the C8-I1 bond longer (2.072 vs $2.049 \AA$ ), which could tentatively be used to rationalize the different reactivity of this reagent.

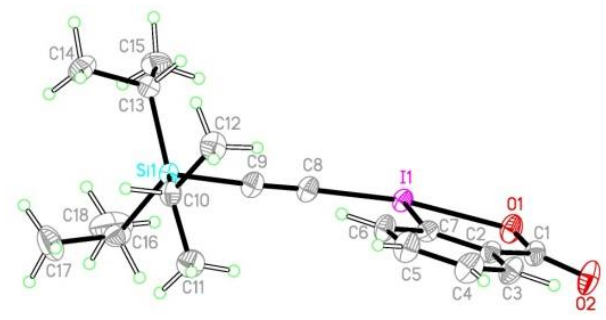

Figure 2. X-ray structure of TIPS-EBX (1). C8-I1 bond: $2.049 \AA$ A . I1-O1 bond: $2.336 \AA$ C8-I1-C7 angle: $91.27^{\circ}$. C8-I1-C7-C6 angle: $5.79^{\circ}$

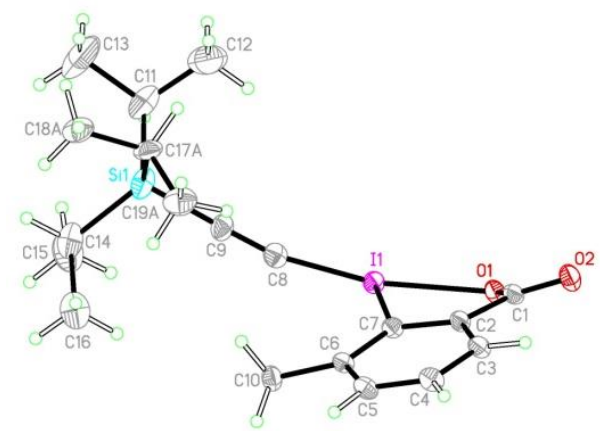

Figure 3. X-ray structure of 3-methylTIPS-EBX (15e). C8-I1 bond: 2.046 A. I1-O1 bond: $2.383 \AA$ A. C8-I1-C7 angle: $95.27^{\circ}$. C8-I1-C7-C6 angle: $34.14^{\circ}$.

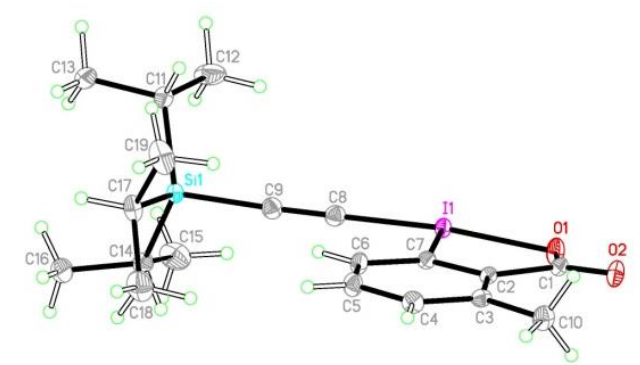

Figure 4. X-ray structure of 6-methylTIPS-EBX (15f). C8-I1 bond: $2.072 \AA$ A. I1-O1 bond: $2.308 \AA$ A. C8-I1-C7 angle: $91.63^{\circ}$. C8-I1-C7-C6 angle: $-0.68^{\circ}$.

With the more reactive reagent $\mathbf{1 5 f}$, a higher yield was obtained both for indole $\mathbf{2 7}$ and pyrrole $\mathbf{2 8}$ (Figure 5).

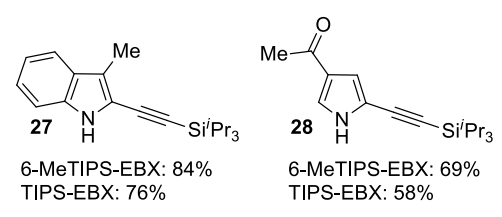

Figure 5. Improved yield using 6-methylTIPS-EBX (15f).

The fact that gold(III) is active for the reaction is mechanistically highly interesting. It seems to indicate that a $\pi$ - 
activation mechanism is more probable. Nevertheless, in situ reduction of $\mathrm{Au}(\mathrm{III})$ to $\mathrm{Au}(\mathrm{I})$ can be envisaged. ${ }^{[38]}$ When TIPS-EBX (1) was mixed with a stoichiometric amount of $\mathrm{AuCl}$, the reagent was transformed into iodobenzoic acid (18) and bisalkyne 29 (Equation 6).

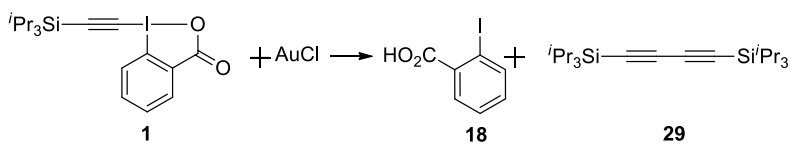

On the contrary, when complex $\mathbf{2 6}$ was used, no reaction was observed (Equation 7). When $\mathbf{2 6}$ was mixed up with indole (6), the fast precipitation of a solid was observed, which was identified as 2pyridine carboxylic acid (30) (Equation 8). Interestingly, the resulting reaction mixture gave product when TIPS-EBX (1) was added, indicating that the reaction between $\mathrm{Au}(\mathrm{III})$ complex 26 and indole (6) indeed formed an active catalyst. A possible explanation is that indole (6) is electron-rich enough to reduce $\mathrm{Au}(\mathrm{III})$ to $\mathrm{Au}(\mathrm{I})$ in situ. Up to now, no product resulting from the oxidation of indole (6), such as indole dimers could be observed by NMR, and further investigations will be required to understand what is happening in this reaction. Furthermore, gold 2-pyridinecarboxylate dichloride (26) gave no product in the case of 2-hexylthiophene $(\mathbf{8})$, which would be in accordance with the lower reduction potential of thiophenes.

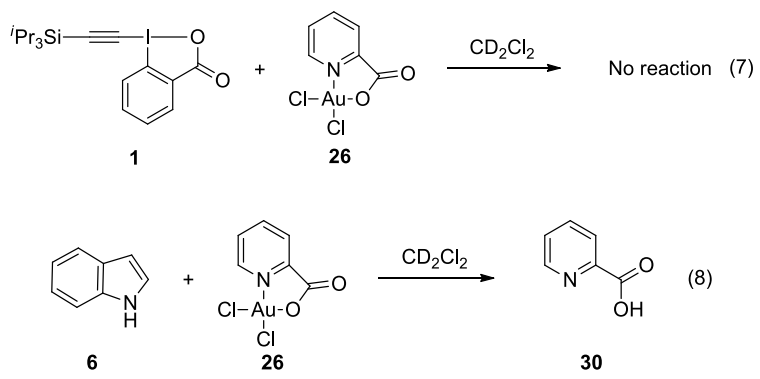

With the results of these control experiments, the fact that $\mathrm{Au}(\mathrm{III})$ is active for the alkynylation of indole unfortunately does not allow the conclusion that a redox mechanism is improbable, as reduction to $\mathrm{Au}(\mathrm{I})$ could occur in situ.

\section{Conclusion}

In this full account, we have reported a more efficient protocol for the alkynylation of pyrroles, which gave high yields even in the case of challenging tetrasubstituted pyrroles. The structure of the ethynylbenziodoxolone has been systematically modified, and in the case of thiophene, the transfer of arylacetylenes has been achieved for the first time. Important conclusions could already be drawn on the reaction mechanism: (1) A mechanism involving attack on iodine or SET processes is less probable. (2) Electrophilic attack on the indole is rate limiting, as demonstrated by competitive experiments (3) Re-aromatization via proton transfer is fast, as no significant kinetic isotope effect could be observed. We further demonstrated that the alkynylation of indole (6) could also be catalyzed by $\mathrm{Au}(\mathrm{III})$ complex $\mathbf{2 6}$, and in this case a more reproducible reaction kinetic was observed. Electron-withdrawing groups and a methyl group in 3 and 6 positions accelerated the reaction. Control experiments showed that the $\mathrm{Au}(\mathrm{III})$ catalyst reacted with indole (6) to form a potentially reduced, not yet identified gold species. This last result does not permit to exclude a mechanism involving changes of oxidation state on gold. In conclusion, the results obtained concerning the influence of the reagent structure, the reaction kinetics and the oxidation state of gold can be used to further support both a redox cycle or a simple $\pi$ activation mechanism. In depth investigation will be required to distinguish definitively these two alternatives and further develop this promising research area.

\section{Experimental Section}

General procedure for pyrrole alkynylation using pyridine:

$\mathrm{AuCl}$ ( $2.3 \mathrm{mg}, 0.010 \mathrm{mmol}, 0.05$ equiv) was added to a stirring solution of pyridine (19 $\mu \mathrm{L}, 0.024 \mathrm{mmol}, 1.2$ equiv), TIPS-EBX (1) $(103 \mathrm{mg}, 0.240 \mathrm{mmol}, 1.2$ equiv) and the corresponding pyrrole $\left(0.200 \mathrm{mmol}, 1.0\right.$ equiv) in $\mathrm{Et}_{2} \mathrm{O}^{[39]}(4 \mathrm{~mL})$ under air. The reaction was sealed and stirred at room temperature for $15 \mathrm{~h} . \mathrm{Et}_{2} \mathrm{O}(10 \mathrm{~mL})$ was added, the organic layer was washed twice with $0.1 \mathrm{M} \mathrm{NaOH}(15 \mathrm{~mL})$. The aqueous layers were combined and extracted with $\mathrm{Et}_{2} \mathrm{O}(20 \mathrm{~mL})$. The organic layers were combined, washed with saturated $\mathrm{NaHCO}_{3}(20 \mathrm{~mL})$, brine $(20 \mathrm{~mL})$, dried with $\mathrm{MgSO}_{4}$ and concentrated under reduced pressure.

1-Methyl-2-((triisopropylsilyl)ethynyl)-1H-pyrrole

(3a),

1-Methyl-3((triisopropylsilyl)ethynyl)-1H-pyrrole (3a')

Following the general procedure but using $2 \mathrm{a}(53 \mu \mathrm{L}, 0.60 \mathrm{mmol}, 3$ equiv) and $\mathbf{1}$ (85 $\mathrm{mg}, 0.20 \mathrm{mmol}, 1$ equiv). Purification by flash chromatography (PET) afforded 3a (37 $\mathrm{mg}, 0.14 \mathrm{mmol}, 71 \%)$ as a colorless oil and $\mathbf{3 a} \mathbf{a}^{\prime}(9.0 \mathrm{mg}, 0.034 \mathrm{mmol}, 17 \%)$ as yellow oil. 3a: $\mathrm{R}_{f}(\mathrm{PET}): 0.1 .{ }^{1} \mathrm{H} \mathrm{NMR}\left(\mathrm{CDCl}_{3}, 400 \mathrm{MHz}\right) \delta 6.62(\mathrm{dd}, J=2.6,1.9 \mathrm{~Hz}, 1 \mathrm{H}$, $\mathrm{ArH}), 6.44(\mathrm{~m}, 1 \mathrm{H}, \mathrm{ArH}), 6.06(\mathrm{~m}, 1 \mathrm{H}, \mathrm{ArH}), 3.69\left(\mathrm{~d}, J=0.3 \mathrm{~Hz}, 3 \mathrm{H}, \mathrm{CH}_{3}\right), 1.14(\mathrm{~m}$, $21 \mathrm{H}, \mathrm{TIPS}) .{ }^{13} \mathrm{C}$ NMR $\left(\mathrm{CDCl}_{3}, 100 \mathrm{MHz}\right) \delta 125.5,123.4,115.0,107.8,98.4,94.5,34.6$, 18.7, 11.4. 3a': $\mathrm{R} f$ (PET): $0.4 .{ }^{1} \mathrm{H}$ NMR $\left(\mathrm{CDCl}_{3}, 400 \mathrm{MHz}\right) \delta 6.83(\mathrm{~m}, 1 \mathrm{H}, \mathrm{ArH}), 6.47(\mathrm{t}$ $J=2.2 \mathrm{~Hz}, 1 \mathrm{H}, \mathrm{ArH}), 6.24(\mathrm{~m}, 1 \mathrm{H}, \mathrm{ArH}), 3.61\left(\mathrm{~s}, 3 \mathrm{H}, \mathrm{CH}_{3}\right), 1.10(\mathrm{~m}, 21 \mathrm{H}, \mathrm{TIPS})$. ${ }^{13} \mathrm{C} \mathrm{NMR}\left(\mathrm{CDCl}_{3}, 100 \mathrm{MHz}\right) \delta 126.3,121.5,112.5,104.8,102.6,87.5,36.3,18.7,11.5$. [13a]

2-Ethyl-5-((triiso-propylsilyl)ethynyl)-1H-pyrrole (3b)

Following the general procedure. Purification by flash chromatography (PET to PET/ $\mathrm{CH}_{2} \mathrm{Cl}_{2}$ 99/1) afforded $\mathbf{3 b}(45 \mathrm{mg}, 0.16 \mathrm{mmol}, 81 \%)$ as colorless oil. $\mathrm{R}_{f}\left(\mathrm{PET} / \mathrm{CH}_{2} \mathrm{Cl}_{2}\right.$ 99/1): 0.1. ${ }^{1} \mathrm{H}$ NMR $\left(\mathrm{CDCl}_{3}, 400 \mathrm{MHz}\right) \delta 8.04(\mathrm{br} \mathrm{s}, 1 \mathrm{H}, \mathrm{NH}), 6.40(\mathrm{dd}, J=3.4,2.9 \mathrm{~Hz}$, $1 \mathrm{H}, \mathrm{ArH}), 5.86(\mathrm{~m}, 1 \mathrm{H}, \mathrm{ArH}), 2.62\left(\mathrm{q}, J=7.7 \mathrm{~Hz}, 2 \mathrm{H}, \mathrm{CH}_{2}\right), 1.25(\mathrm{t}, J=7.6 \mathrm{~Hz}, 3 \mathrm{H}$, $\left.\mathrm{CH}_{3}\right), 1.12\left(\mathrm{~m}, 21 \mathrm{H}\right.$, TIPS). ${ }^{13} \mathrm{C} \mathrm{NMR}\left(\mathrm{CDCl}_{3}, 100 \mathrm{MHz}\right) \delta 135.9,115.8,111.7,105.5$, $99.5,91.3,21.0,18.7,13.5,11.4{ }^{[13 a]}$

2-Phenyl-5-((triiso-propylsilyl)ethynyl)-1H-pyrrole (3c)

Following the general procedure. On $0.49 \mathrm{mmol}$. Purification by flash chromatography (PET/ $\mathrm{CH}_{2} \mathrm{Cl}_{2}$ 95/5) afforded 3c (133 mg, $\left.0.411 \mathrm{mmol}, 84 \%\right)$ as amorphous solid. $\mathrm{R}$ (PET/Et $\left.{ }_{2} \mathrm{O} 5 / 95\right): 0.2 .{ }^{1} \mathrm{H}$ NMR $\left(\mathrm{CDCl}_{3}, 400 \mathrm{MHz}\right) \delta 8.54(\mathrm{br} \mathrm{s}, 1 \mathrm{H}, \mathrm{NH}), 7.52(\mathrm{~m}, 2 \mathrm{H}$, $\operatorname{ArH}), 7.41(\mathrm{~m}, 2 \mathrm{H}, \mathrm{ArH}), 7.28(\mathrm{~m}, 1 \mathrm{H}, \mathrm{ArH}), 6.60(\mathrm{dd}, J=2.2,3.4 \mathrm{~Hz}, 1 \mathrm{H}, \mathrm{ArH})$, $6.50(\mathrm{dd}, J=2.7,3.6 \mathrm{~Hz}, 1 \mathrm{H}, \mathrm{ArH}), 1.19(\mathrm{~m}, 21 \mathrm{H}, \mathrm{TIPS}) .{ }^{13} \mathrm{C} \mathrm{NMR}\left(\mathrm{CDCl}_{3}, 100 \mathrm{MHz}\right)$ $\delta 133.1,131.9,129.0,126.9,124.2,117.3,114.3,106.8,99.0,93.1,18.8,11.4 .{ }^{[13 a]}$

3,5-dimethyl-2-((triisopropylsilyl)ethynyl)- $1 \mathrm{H}$-pyrrole (3d) and 2,4-dimethyl-3,5bis((triisopropylsilyl)ethynyl)-1H-pyrrole (31)

Following the general procedure. Purification by flash chromatography (PET/ $\mathrm{CH}_{2} \mathrm{Cl}_{2}$ $95 / 5)$ afforded $3 \mathbf{d}(31 \mathrm{mg}, 0.11 \mathrm{mmol}, 56 \%)$ as colorless oil and $\mathbf{3 1}(14 \mathrm{mg}, 0.031$ mmol, $15 \%$ ) as colorless oil.

With 3 equivalent of TIPS-EBX (1) $(258 \mathrm{mg}, 0.600 \mathrm{mmol})$ purification by flash chromatography (PET/ $\mathrm{CH}_{2} \mathrm{Cl}_{2}$ 95/5) afforded $\mathbf{3 1}(66 \mathrm{mg}, 0.15 \mathrm{mmol}, 73 \%)$ as colorless oil. 3d: $\mathrm{R}_{f}$ (PET/ $\mathrm{CH}_{2} \mathrm{Cl}_{2}$ 95/5): 0.2. ${ }^{1} \mathrm{H}$ NMR (400 MHz, $\left.\mathrm{CDCl}_{3}\right) \delta 7.80$ (br s, $1 \mathrm{H}, \mathrm{NH}$ ), $5.70(\mathrm{~s}, 1 \mathrm{H}, \mathrm{ArH}), 2.20\left(\mathrm{~s}, 3 \mathrm{H}, \mathrm{CH}_{3}\right), 2.12\left(\mathrm{~m}, 3 \mathrm{H}, \mathrm{CH}_{3}\right), 1.11(\mathrm{~m}, 21 \mathrm{H}, \mathrm{TIPS}) .{ }^{13} \mathrm{C}$ $\operatorname{NMR}\left(101 \mathrm{MHz}, \mathrm{CDCl}_{3}\right) \delta 128.7,127.0,110.1,108.3,99.0,93.7,18.7,13.1,11.7,11.3$. IR 3477 (w), 3377 (w), 2941 (s), 2924 (m), 2864 (s), 2137 (s), 1718 (w), 1583 (w), 1493 (w), $1451(w), 1382(w), 1351(w), 1299(w), 1245(w), 1146(w), 1073(w), 1017(w)$, $996(\mathrm{~m}), 919(\mathrm{w}), 883(\mathrm{~m}), 838(\mathrm{w}), 812(\mathrm{~m}), 791(\mathrm{w}), 701(\mathrm{~m}), 677(\mathrm{~s}), 650(\mathrm{~m})$. HRMS (ESI) calcd for $\mathrm{C}_{17} \mathrm{H}_{30} \mathrm{NSi}^{+}[\mathrm{M}+\mathrm{H}]^{+}$276.2142; found 276.2135. 31: Rf 0.1 (PET/ $\mathrm{CH}_{2} \mathrm{Cl}_{2}$ 95/5). ${ }^{1} \mathrm{H}$ NMR (400 MHz, $\left.\mathrm{CDCl}_{3}\right) \delta 7.84$ (br s, $\left.1 \mathrm{H}, \mathrm{NH}\right), 2.29$ (s, $3 \mathrm{H}, \mathrm{Me}$ ), $2.15(\mathrm{~m}, 3 \mathrm{H}, \mathrm{Me}), 1.12\left(\mathrm{~m}, 42 \mathrm{H}\right.$, TIPS). ${ }^{13} \mathrm{C} \mathrm{NMR}\left(101 \mathrm{MHz}, \mathrm{CDCl}_{3}\right) \delta 133.3,128.9$, 109.5, 104.7, 101.0, 98.1, 94.1, 92.0, 18.7, 18.7, 12.3, 11.3, 11.3, 10.8. IR $3472(w)$, 3377 (w), 2942 (s), 2865 (s), 2140 (s), 1716 (w), 1579 (w), 1383 (w), 1298 (w), 1206 (w), 1132 (w), 1073 (w), 1017 (w), 996 (w), 889 (w), 865 (w), 812 (w), 780 (w), 713 (w), 674 (s). HRMS (ESI) calcd for $\mathrm{C}_{28} \mathrm{H}_{50} \mathrm{NSi}_{2}{ }^{+}[\mathrm{M}+\mathrm{H}]^{+} 456.3476$; found 456.3491 
Following the general procedure. Purification by flash chromatography ( $\mathrm{PET} / \mathrm{Et}_{2} \mathrm{O}$ 85/15) afforded 3e (40 mg, $0.15 \mathrm{mmol}, 73 \%)$ as yellow oil. $\mathrm{R}_{f}\left(\mathrm{PET}_{\mathrm{Et}} \mathrm{O}\right.$ 85:15): 0.3 . ${ }^{1} \mathrm{H} \mathrm{NMR}\left(\mathrm{CDCl}_{3}, 400 \mathrm{MHz}\right) \delta 7.58(\mathrm{br} \mathrm{s}, 1 \mathrm{H}, \mathrm{NH}), 5.90(\mathrm{~d}, J=1.7 \mathrm{~Hz}, 1 \mathrm{H}, \mathrm{ArH}), 2.32$ (s, $3 \mathrm{H}, \mathrm{Me}), 2.20$ (s, $3 \mathrm{H}, \mathrm{Me}), 1.14(\mathrm{~m}, 21 \mathrm{H}, \mathrm{TIPS}) .{ }^{13} \mathrm{C} \mathrm{NMR}\left(\mathrm{CDCl}_{3}, 100 \mathrm{MHz}\right) \delta$ $132.3,125.5,108.8,103.1,102.3,89.1,18.8,12.8,12.0,11.5{ }^{[13 a]}$

\section{1,2,5-Trimethyl-3-((triisopropylsilyl)ethynyl)-1H-pyrrole (3f)}

Following the general procedure. Purification by flash chromatography (PET/ $\mathrm{CH}_{2} \mathrm{Cl}_{2}$ 95/5) afforded $3 f(56 \mathrm{mg}, 0.19 \mathrm{mmol}, 97 \%)$ as colorless oil. $\mathrm{Rf} 0.3\left(\mathrm{PET} / \mathrm{CH}_{2} \mathrm{Cl}_{2} 95 / 5\right)$. ${ }^{1} \mathrm{H}$ NMR $\left(400 \mathrm{MHz}, \mathrm{CDCl}_{3}\right) \delta 5.92(\mathrm{~s}, 1 \mathrm{H}, \mathrm{ArH}), 3.36\left(\mathrm{~s}, 3 \mathrm{H}, \mathrm{CH}_{3}\right), 2.29\left(\mathrm{~s}, 3 \mathrm{H}, \mathrm{CH}_{3}\right)$ $2.16\left(\mathrm{~s}, 3 \mathrm{H}, \mathrm{CH}_{3}\right), 1.12\left(\mathrm{~m}, 21 \mathrm{H}\right.$, TIPS). ${ }^{13} \mathrm{C}$ NMR $\left(101 \mathrm{MHz}, \mathrm{CDCl}_{3}\right) \delta 133.7,127.3$, 108.0, 103.4, 101.0, 88.7, 30.5, 18.7, 12.2, 11.4, 11.3. IR 2957 (m), $2939(\mathrm{~m}), 2863(\mathrm{~m})$ 1533 (w), 1462 (m), 1439 (w), 1417 (w), 1389 (w), 1349 (m), 1243 (w), 1190 (w), 110 (w), 1074 (w), 1015 (w), 999 (m), 918 (w), 882 (s), 839 (w), 769 (m), 735 (w), 701 (s), 657 (s). HRMS (ESI) calcd for $\mathrm{C}_{18} \mathrm{H}_{32} \mathrm{NSi}^{+}[\mathrm{M}+\mathrm{H}]^{+} 290.2299$; found 290.2298 .

\section{3-Ethyl-2,4-dimethyl-5-((triiso-propylsilyl)ethynyl)-1H-pyrrole (3g)}

Following the general procedure. Purification by flash chromatography $\left(\mathrm{PET} / \mathrm{CH}_{2} \mathrm{Cl}_{2}\right.$ 99/1) afforded $\mathbf{3 g}$ (50 mg, $0.17 \mathrm{mmol}, 83 \%)$ as yellow oil. $\mathrm{R}_{f}\left(\mathrm{PET} / \mathrm{CH}_{2} \mathrm{Cl}_{2}\right.$ 99/1): 0.25. ${ }^{1} \mathrm{H} \mathrm{NMR}\left(\mathrm{CDCl}_{3}, 400 \mathrm{MHz}\right) \delta 7.74$ (br s, $\left.1 \mathrm{H}, \mathrm{NH}\right), 2.40\left(\mathrm{q}, J=7.6 \mathrm{~Hz}, 2 \mathrm{H}, \mathrm{CH}_{2}\right), 2.19$ (s, $3 \mathrm{H}, \mathrm{Me}$ ), 2.14 (s, $3 \mathrm{H}, \mathrm{Me}$ ), 1.15 (m, $21 \mathrm{H}$, TIPS), $1.08\left(\mathrm{t}, J=7.7 \mathrm{~Hz}, 3 \mathrm{H}, \mathrm{CH}_{2} \mathrm{CH}_{3}\right.$ ) ${ }^{13} \mathrm{C} \mathrm{NMR}\left(\mathrm{CDCl}_{3}, 100 \mathrm{MHz}\right) \delta 125.5,124.7,121.4,108.9,99.4,93.6,18.8,17.6,15.4$, $11.4,11.2,10.0 .^{[13 a]}$

\section{1-[Phenylethynyl]-1,2-benziodoxol-3(1H)-one (Ph-EBX, 5e)}

Trimethylsilyl triflate $(7.50 \mathrm{~mL}, 41.5 \mathrm{mmol}, 1.1$ equiv) was added to a suspension of 2 iodosylbenzoic acid (4) (10.0 g, $37.7 \mathrm{mmol}, 1$ equiv) in $\mathrm{CH}_{2} \mathrm{Cl}_{2}(100 \mathrm{~mL})$ at $\mathrm{RT}$. The resulting yellow mixture was stirred for $1 \mathrm{~h}$, followed by the dropwise addition of trimethyl(phenylethynyl)silane ( $8.10 \mathrm{~mL}, 41.5 \mathrm{mmol}, 1.1 \mathrm{equiv})$ (slightly exothermic) The resulting suspension was stirred for $6 \mathrm{~h}$ at RT, during this time a white solid was formed. A saturated solution of $\mathrm{NaHCO}_{3}(100 \mathrm{~mL})$ was then added and the mixture was stirred vigorously. The resulting suspension was filtered on a glass filter of porosity 4 The two layers of the mother liquors were separated and the organic layer was washed with sat. $\mathrm{NaHCO}_{3}(100 \mathrm{~mL})$, dried over $\mathrm{MgSO}_{4}$, filtered and evaporated under reduced pressure. The resulting mixture was combined with the solid obtained by filtration and boiled in $\mathrm{CH}_{3} \mathrm{CN}(300 \mathrm{~mL})$. The mixture was cooled down, filtered and dried unde high vacuum to afford $\mathbf{5 e}(6.08 \mathrm{~g}, 17.4 \mathrm{mmol}, 46 \%)$ as a colorless solid. Mp (Dec.) 155 $-160^{\circ} \mathrm{C}$ (lit $\left.153-155^{\circ} \mathrm{C}\right) .{ }^{1} \mathrm{H}$ NMR $\left(400 \mathrm{MHz}, \mathrm{CDCl}_{3}\right)(\mathrm{ca} 0.03 \mathrm{mmol} / \mathrm{ml}) \delta 8.46(\mathrm{~m}, 1$ $\mathrm{H}, \mathrm{ArH}), 8.28$ (m, $1 \mathrm{H}, \mathrm{ArH}), 7.80(\mathrm{~m}, 2 \mathrm{H}, \mathrm{ArH}), 7.63(\mathrm{~m}, 2 \mathrm{H}, \mathrm{ArH}), 7.48(\mathrm{~m}, 3 \mathrm{H}$, ArH). ${ }^{13} \mathrm{C}$ NMR (101 MHz, $\left.\mathrm{CDCl}_{3}\right) \delta 163.9,134.9,132.9,132.5,131.6,131.3 .130 .8$, $128.8,126.2,120.5,116.2,106.6,50.2{ }^{[17]}$

\section{3-Methyl-1-[(triisopropylsilyl)ethynyl]-1,2-benziodoxol-3(1H)-one (15e)}

Trimethylsilyltriflate $(2.10 \mathrm{~mL}, 11.6 \mathrm{mmol}, 1.1$ equiv) was added dropwise to a stirred solution of 2-iodosylbenzoic acid (4) $(2.93 \mathrm{~g}, 10.5 \mathrm{mmol}, 1.0$ equiv) in acetonitrile (45 $\mathrm{mL}$ ). After $20 \mathrm{~min}$, (trimethylsilyl)(triiso-propylsilyl)acetylene (14) $(2.94 \mathrm{~g}, 11.6 \mathrm{mmol}$, 1.1 equiv) was then added dropwise, followed, after $30 \mathrm{~min}$, by the addition of pyridine ( $934 \mu \mathrm{L}, 11.6 \mathrm{mmol}, 1.1$ equiv). The mixture was stirred $20 \mathrm{~min}$. The solvent was then removed under reduced pressure and the yellow crude oil was dissolved in dichloromethane $(30 \mathrm{~mL})$. The organic layer was washed with $1 \mathrm{M} \mathrm{HCl}(20 \mathrm{~mL})$ and the aqueous layer was extracted with $\mathrm{CH}_{2} \mathrm{Cl}_{2}(30 \mathrm{~mL})$. The organic layers were combined, washed with a saturated solution of $\mathrm{NaHCO}_{3}(40 \mathrm{~mL})$, dried over $\mathrm{MgSO}_{4}$ filtered and the solvent was evaporated under reduced pressure. Recrystallization from acetonitrile $(\mathrm{ca} 10 \mathrm{~mL})$ and wash with pentane afforded $15 \mathrm{e}(2.79 \mathrm{~g}, 6.31 \mathrm{mmol}, 60 \%)$ as colorless cristals. Mp (Dec.) $138-145^{\circ} \mathrm{C} .{ }^{1} \mathrm{H} \mathrm{NMR}\left(400 \mathrm{MHz}, \mathrm{CDCl}_{3}\right)(\mathrm{ca} 0.04$ $\mathrm{mmol} / \mathrm{mL}) \delta 8.21(\mathrm{dd}, 1 \mathrm{H}, J=6.8,2.5 \mathrm{~Hz}, \operatorname{ArH}), 7.50(\mathrm{~m}, 2 \mathrm{H}, \mathrm{ArH}), 2.87(\mathrm{~s}, 3 \mathrm{H}$, $\left.\mathrm{CH}_{3}\right), 1.10$ (m, $21 \mathrm{H}$, TIPS). ${ }^{13} \mathrm{C}$ NMR (101 MHz, $\left.\mathrm{CDCl}_{3}\right) \delta 166.8,140.3,138.0,133.3$, 131.7, 130.8, 119.1, 112.5, 66.9, 24.0, 18.5, 11.2. IR 2946 (w), 2867 (w), $2244(w)$, $1649(\mathrm{~m}), 1562(\mathrm{w}), 1464(\mathrm{w}), 1326(\mathrm{w}), 1281(\mathrm{w}), 998(\mathrm{w}), 907(\mathrm{~s}), 884(\mathrm{w}), 763(\mathrm{w})$ $728(\mathrm{~s}), 687(\mathrm{~s}), 647(\mathrm{~m})$. HRMS(ESI) calcd for $\mathrm{C}_{19} \mathrm{H}_{28} \mathrm{O}_{2} \mathrm{ISi}^{+}(\mathrm{M}+\mathrm{H}) 443.0903$, found 443.0893 .

\section{6-Methyl-1-[(triisopropylsilyl)ethynyl]-1,2-benziodoxol-3(1H)-one (15f)}

Trimethylsilyltriflate $(1.50 \mathrm{~mL}, 8.27 \mathrm{mmol}, 1.1$ equiv, freshly distilled) was added dropwise to a stirred solution of 2-iodosylbenzoic acid (4) $(2.09 \mathrm{~g}, 7.52 \mathrm{mmol}, 1.0$ equiv) in acetonitrile $(30 \mathrm{~mL}$ ). After $20 \mathrm{~min}$, (trimethylsilyl)(triiso-propylsilyl)acetylene (14) $(2.10 \mathrm{~g}, 8.27 \mathrm{mmol}, 1.1$ equiv) was then added dropwise, followed, after $20 \mathrm{~min}$, by the addition of pyridine ( $667 \mu \mathrm{L}, 8.27 \mathrm{mmol}, 1.1$ equiv). The mixture was stirred $20 \mathrm{~min}$. The solvent was then removed under reduced pressure and the yellow crude oil was dissolved in dichloromethane $(150 \mathrm{~mL})$. The organic layer was washed with $1 \mathrm{M} \mathrm{HCl}$ $(150 \mathrm{~mL})$ and the aqueous layer was extracted with $\mathrm{CH}_{2} \mathrm{Cl}_{2}(150 \mathrm{~mL})$. The organic layers were combined, washed with a saturated solution of $\mathrm{NaHCO}_{3}(150 \mathrm{~mL})$, dried over $\mathrm{MgSO}_{4}$, filtered and the solvent was evaporated under reduced pressure. Recrystallization from acetonitrile and wash with cold acetonitrile afforded $\mathbf{1 5 f}(2.84 \mathrm{~g}$, $6.60 \mathrm{mmol}, 88 \%)$ as colorless cristals. Mp: $123-125^{\circ} \mathrm{C} .{ }^{1} \mathrm{H}$ NMR $\left(400 \mathrm{MHz}, \mathrm{CDCl}_{3}\right) \delta$ $8.25(\mathrm{~m}, 1 \mathrm{H}, \mathrm{ArH}), 7.53(\mathrm{~d}, 2 \mathrm{H}, J=5.2 \mathrm{~Hz}, \mathrm{ArH}), 2.90\left(\mathrm{~s}, 3 \mathrm{H}, \mathrm{CH}_{3}\right), 1.15(\mathrm{~m}, 21 \mathrm{H}$, TIPS). ${ }^{13} \mathrm{C} \mathrm{NMR}\left(101 \mathrm{MHz}, \mathrm{CDCl}_{3}\right) \delta 166.8,146.7,135.0,133.3,128.7,124.2,118.3$, 113.3, 68.7, 22.4, 18.5, 11.2. IR $3055(\mathrm{w}), 2938(\mathrm{~m}), 2873(\mathrm{~m}), 2865(\mathrm{~m}), 2244(\mathrm{w})$, 2089 (w), 1626 (s), 1612 (s), $1586(\mathrm{~m}), 1550$ (m), $1450(\mathrm{~m}), 1382(\mathrm{w}), 1329(\mathrm{~m}), 1276$ (w), $1253(\mathrm{w}), 1157(\mathrm{w}), 1076(\mathrm{w}), 1018(\mathrm{w}), 998(\mathrm{w}), 911(\mathrm{w}), 884(\mathrm{~m}), 846(\mathrm{~m}), 817$ $(\mathrm{m}), 770(\mathrm{~m}), 706(\mathrm{~s}), 679(\mathrm{~s}), 649(\mathrm{~m})$. HRMS (ESI) calcd for $\mathrm{C}_{19} \mathrm{H}_{28} \mathrm{IO}_{2} \mathrm{Si}^{+}[\mathrm{M}+\mathrm{H}]^{+}$ 443.0898; found 443.0896..

\section{1-[(Triisopropylsilyl)ethynyl]-3,3-dimethyl-3(1H)-1,2-benziodoxole (23)}

Trimethylsilyltriflate ( $250 \mu \mathrm{L}, 1.38 \mathrm{mmol}, 1$ equiv) was added to a stirring solution of $\mathbf{4}$ (408 mg, $1.38 \mathrm{mmol}, 1$ equiv) in THF ( $40 \mathrm{~mL}$ ) at RT. The solution was stirred at RT for $20 \mathrm{~min}$ and then cooled to $-78^{\circ} \mathrm{C}$. In the meantime, ${ }^{n} \mathrm{BuLi}(2.5 \mathrm{M}$ in hexanes, $550 \mu \mathrm{L}$, $1.38 \mathrm{mmol}, 1$ equiv) was added to a stirring solution of triisopropylacetylene $(310 \mu \mathrm{L}$, $1.38 \mathrm{mmol}, 1$ equiv) in THF $(10 \mathrm{~mL})$ at $-78^{\circ} \mathrm{C}$. The solution was stirred for $30 \mathrm{~min}$ at $78^{\circ} \mathrm{C}$ and then added via cannula to the first solution. The reaction was stirred for $1 \mathrm{~h}$ at $-78^{\circ} \mathrm{C}$, warmed to $\mathrm{RT}$ and stirred $4 \mathrm{~h}$. The reaction was quenched with saturated $\mathrm{NH}_{4} \mathrm{Cl}$ $(20 \mathrm{~mL})$. The layers were separated and the aqueous layers were extracted with $\mathrm{CH}_{2} \mathrm{Cl}_{2}$ $(20 \mathrm{~mL})$. The organic layers were combined, washed with brine, dried over $\mathrm{MgSO}_{4}$, filtered and reduced under vacuum. The resulting oil was then purified by column chromatography (PET/Et $2 \mathrm{O} 6 / 4)$ to afford $23(524 \mathrm{mg}, 1.18 \mathrm{mmol}, 86 \%)$ as a yellow oil which cristalize at $-18^{\circ} \mathrm{C}$. $\mathrm{R}_{\mathrm{f}} \mathrm{PET} / \mathrm{Et}_{2} \mathrm{O}$ 6/4: 0.15 . Mp $59-61^{\circ} \mathrm{C} .{ }^{1} \mathrm{H} \mathrm{NMR}(400 \mathrm{MHz}$, $\left.\mathrm{CDCl}_{3}\right)(c a 0.16 \mathrm{mmol} / \mathrm{mL}) \delta 8.23(\mathrm{dd}, 1 \mathrm{H}, J=8.2,0.9 \mathrm{~Hz}, \mathrm{ArH}), 7.52(\mathrm{td}, 1 \mathrm{H}, J=7.3$ $1.0 \mathrm{~Hz}, \mathrm{ArH}), 7.41$ (ddd, $1 \mathrm{H}, J=8.6,7.2,1.5 \mathrm{~Hz}, \mathrm{ArH}), 7.35(\mathrm{dd}, 1 \mathrm{H}, J=7.5,1.5 \mathrm{~Hz}$, $\mathrm{ArH}), 1.44$ (s, $6 \mathrm{H}, \mathrm{Me}), 1.12$ (s, $21 \mathrm{H}, \mathrm{TIPS}) .{ }^{13} \mathrm{C}$ NMR (101 MHz, $\left.\mathrm{CDCl}_{3}\right) \delta 148.0$, 130.4, 129.2, 127.4, 126.5, 111.0, 105.8, 80.8, 75.7, 31.5, 18.6, 11.4. IR $2945(\mathrm{~m}), 2864$ (m), $2064(\mathrm{w}), 1690(\mathrm{w}), 1562(\mathrm{w}), 1462(\mathrm{~m}), 1436(\mathrm{~m}), 1355(\mathrm{w}), 1244(\mathrm{w}), 1162(\mathrm{w})$, $1116(\mathrm{w}), 1073(\mathrm{w}), 999(\mathrm{~m}), 968(\mathrm{~m}), 883(\mathrm{~m}), 756(\mathrm{~m}), 691$ (s). HRMS(ESI) calcd for $\mathrm{C}_{20} \mathrm{H}_{32} \mathrm{OISi}^{+}(\mathrm{M}+\mathrm{H})$ 443.1267, found 443.1276.

\section{3-Methyl-2-((triisopropylsilyl)ethynyl)-1H-indole (27)}

$\mathrm{AuCl}$ ( $2.3 \mathrm{mg}, 0.020 \mathrm{mmol}, 0.05$ equiv) was added to a stirring solution of 6 methylTIPS-EBX (15f) (106 mg, $0.240 \mathrm{mmol}, 1.2$ equiv) and 3-methylindole (26.2 mg, $0.200 \mathrm{mmol}, 1.0$ equiv) in $\mathrm{Et}_{2} \mathrm{O}^{[39]}(4 \mathrm{~mL})$ under air. The reaction was sealed and stirred at room temperature for $14 \mathrm{~h}$. $\mathrm{Et}_{2} \mathrm{O}(10 \mathrm{~mL})$ was added, the organic layer was washed twice with $0.1 \mathrm{M} \mathrm{NaOH}(15 \mathrm{~mL})$. The aqueous layers were combined and extracted with $\mathrm{Et}_{2} \mathrm{O}(20 \mathrm{~mL})$. The organic layers were combined, washed with saturated $\mathrm{NaHCO}_{3}$ $(20 \mathrm{~mL})$, brine $(20 \mathrm{~mL})$, dried with $\mathrm{MgSO}_{4}$ and concentrated under reduced pressure. Purification by column chromatography (pentane $/ \mathrm{CH}_{2} \mathrm{Cl}_{2}$ 95/5) afforded 27 (52 mg, $0.17 \mathrm{mmol}, 84 \%$ ) as an amorphous orange solid. $\mathrm{R}_{f} 0.1$ (pentane/ $\mathrm{CH}_{2} \mathrm{Cl}_{2}$ 95/5). Mp 75$77{ }^{\circ} \mathrm{C} .{ }^{1} \mathrm{H}$ NMR $\left(\mathrm{CDCl}_{3}, 400 \mathrm{MHz}\right) \delta 7.98($ br s, $1 \mathrm{H}, \mathrm{NH}), 7.59(\mathrm{~d}, J=7.9 \mathrm{~Hz}, 1 \mathrm{H}$, $\operatorname{ArH}), 7.28$ (m, $2 \mathrm{H}, \mathrm{ArH}), 7.17(\mathrm{~m}, 1 \mathrm{H}, \mathrm{ArH}), 2.47$ (d, $J=0.5 \mathrm{~Hz}, 3 \mathrm{H}, \mathrm{Me}), 1.23(\mathrm{~m}$, $21 \mathrm{H}, \mathrm{TIPS}) .{ }^{13} \mathrm{C}$ NMR $\left(\mathrm{CDCl}_{3}, 100 \mathrm{MHz}\right) \delta 135.6,127.8,123.7,119.8,119.3,118.9$, $117.0,110.7,98.5,97.4,18.8,11.4,9.6^{[13 a]}$

\section{1-(5-((Triisopropylsilyl)ethynyl)-1H-pyrrol-3-yl)ethanone (28)}

$\mathrm{AuCl}(2.3 \mathrm{mg}, 0.020 \mathrm{mmol}, 0.05$ equiv) was added to a stirring solution of 6 methylTIPS-EBX (15f) (106 mg, $0.240 \mathrm{mmol}, 1.2$ equiv), 3-acetylpyrrole $(22.0 \mathrm{mg}$, $0.200 \mathrm{mmol}, 1.0$ equiv) in $\mathrm{Et}_{2} \mathrm{O}^{[39]}(4 \mathrm{~mL})$ under air. The reaction was sealed and stirred at room temperature for $15 \mathrm{~h}$. $\mathrm{Et}_{2} \mathrm{O}(10 \mathrm{~mL})$ was added and the organic layer was washed twice with $0.1 \mathrm{M} \mathrm{NaOH}(15 \mathrm{~mL})$. The aqueous layers were combined and extracted with $\mathrm{Et}_{2} \mathrm{O}(20 \mathrm{~mL})$. The organic layers were combined, washed with saturated $\mathrm{NaHCO}_{3}(20 \mathrm{~mL})$, brine $(20 \mathrm{~mL})$, dried with $\mathrm{MgSO}_{4}$ and concentrated under reduced pressure. Purification by column chromatography (pentane/ $\mathrm{Et}_{2} \mathrm{O} 7 / 3$ ) afforded 28 (40 $\mathrm{mg}, 0.14 \mathrm{mmol}, 69 \%)$ as a yellow solid. $\mathrm{R}_{f} 0.3\left(\mathrm{PET}_{\mathrm{Et}} \mathrm{O}\right.$ 7/3). Mp 120-122 ${ }^{\circ} \mathrm{C} .{ }^{1} \mathrm{H}$ NMR $\left(\mathrm{CDCl}_{3}, 400 \mathrm{MHz}\right) \delta 9.52(\mathrm{br} \mathrm{s}, 1 \mathrm{H}, \mathrm{NH}), 7.37(\mathrm{dd}, 1 \mathrm{H}, J=2.6,1.2 \mathrm{~Hz}, \mathrm{ArH})$, $6.87(\mathrm{~m}, 1 \mathrm{H}, \mathrm{ArH}), 2.44(\mathrm{~s}, 3 \mathrm{H}, \mathrm{Ac}), 1.10(\mathrm{~m}, 21 \mathrm{H}, \mathrm{TIPS}) .{ }^{13} \mathrm{C} \mathrm{NMR}\left(\mathrm{CDCl}_{3}\right.$, $100 \mathrm{MHz}) \delta 193.7,126.2,124.1,115.3,115.1,97.4,93.4,27.3,18.6,11.3 .^{[13 a]}$

\section{Acknowledgements}

We thank EPFL for funding and F. Hoffmann-La Roche Ltd for an unrestricted research grant. We thank Dr. Fides Benfatti and Dr. Reto Frei for proofreading the manuscript. We thank Dr. Davinia Fernández González for preparation of TMS-EBX (5a).

[1] F. Diederich, P. J. Stang, R. R. Tykwinski, in Acetylene Chemistry: Chemistry, Biology and Material Science; WILEY-VCH, Weinheim, 2005. 
[2] J. M. Tour, $A c c$. Chem Res. 2000, 33, 791. b) J. M. Tour, Chem. Rev 1996, 96 , 537. c) J. S. Moore, Acc. Chem. Res. 1997, 30, 402. d) M. M. Haley, J. J. Pak, S C. Brand, Top. Curr. Chem. 1999, 201, 81. e) W. J. Youngs, C. A. Tessier, J. D. Bradshaw, Chem. Rev. 1999, 99, 3153. f) M. M. Haley Synlett 1998, 557. (g) M. R. Pinto, K. S. Schanze, Synthesis 2002, 1293. h) T. Yamamoto, Synlett 2003 425. i) Y. Tobe, M. Sonoda, in Modern Cyclophane Chemistry; Gleiter, R., Hopf, H., Eds.; WILEY-VCH, Weinheim, 2004.

[3] a) T. M. Swager, in Semiconducting Poly(arylene ethylene)s in Acetylene Chemistry: Chemistry, Biology and Material Science; F. Diederich, P: J: Stang, R. R. Tykwinski, WILEY-VCH, Weinheim, 2005. b) D. K. James, J .M. Tour, Top. Curr. Chem. 2005, 257, 33.

[4] a) D. Seyferth, R. S. Marmor, P. J. Hilbert, Org. Chem. 1971, 36, 1379. b) J. C. Gilbert, U. Weerasooriya, J. Org. Chem. 1982, 47, 1837. c) E. J. Corey, P. L. Fuchs, Tetrahedron Lett. 1972, 3769. For other elimination-type syntheses of aromatic acetylenes, see: d) A. Orita, J. Otera, Chem. Rev. 2006, 106, 5387.

[5] a) K. Sonogashira, Y. Tohda, N. Hagihara, Tetrahedron Lett. 1975, 16, 4467. b) K. Sonogashira, in Handbook of Organopalladium Chemistry for Organic Synthesis; E: Negishi, WILEY-VCH, New York, 2002, p 493. c) K. Sonogashira, J. Organomet. Chem. 2002, 653. d) R. Chinchilla, C. Najera, Chem. Soc. Rev. 2011, 40, 5084

[6] a) D. Alberico, M. E. Scott, M. Lautens, Chem. Rev. 2007, 107, 174. b) I. V. Seregin, V. Gevorgyan, Chem. Soc. Rev. 2007, 36, 1173. c) F. Bellina, R. Rossi Tetrahedron 2009, 65, 10269. d) L. Ackermann, R. Vicente, A. R. Kapdi, Angew. Chem., Int. Ed. 2009, 48, 9792

[7] For non catalytic methods, see: a) B. A. Trofimov, Z. V. Stepanova, L. N Sobenina, A. I. Mikhaleva, I. A. Ushakov, Tetrahedron Lett. 2004, 45, 6513. b) B. A. Trofimov, L. N. Sobenina, Z. V. Stepanova, A. P. Demenev, A. I. Mikhaleva, I. A. Ushakov, T. I. Vakul'skaya, O. V. Petrova, Russ. J. Org. Chem. 2006, 42, 1348. c) B. A. Trofimov, L. N. Sobenina, Z. V. Stepanova, I. A Ushakov, O. V. Petrova, O. A. Tarasova, K. A. Volkova, A. I. Mikhaleva Synthesis 2007, 447. d) B. A. Trofimov, L. N. Sobenina, Z. V. Stepanova, T. I. Vakul'skaya, O. N. Kazheva, G. G. Aleksandrov, O. A. Dyachenko, A. I. Mikhaleva, Tetrahedron 2008, 64, 5541. Using a stoichiometric amount of $\mathrm{Cu}^{\prime}$ complex: e) K. N. Kalinin, D. N. Pashchenko, F. M. She, Mendeleev Commun. 1992, 60 .

[8] a) K. Kobayashi, M. Arisawa, M. Yamaguchi, J. Am. Chem. Soc. 2002, 124 8528. b) R. Amemiya, A. Fujii, M. Arisawa, M. Yamaguchi, J. Organomet. Chem. 2003, 686, 94

[9] I. V. Seregin, V. Ryabova, V. Gevorgyan, J. Am. Chem. Soc. 2007, 129, 7742

[10] Reviews: a) A. S. Dudnik, V. Gevorgyan, V. Angew. Chem. Int., Ed. 2010, 49 2096. b) S. Messaoudi, J. D. Brion, M. Alami, Eur. J. Org. Chem. 2010, 6495.

[11] a) N. Matsuyama, K. Hirano, T. Satoh, M. Miura, Org. Lett. 2009, 11, 4156. b) T. Kawano, N. Matsuyama, K. Hirano, T. Satoh, M. Miura, J. Org. Chem. 2010 75, 1764. c) M. Kitahara, K. Hirano, H. Tsurugi, T. Satoh, M. Miura, M. Chem Eur. J. 2010, 16, 1772. d) N. Matsuyama, M. Kitahara, K. Hirano, T. Satoh, M Miura, Org. Lett. 2010, 12, 2358. e) F. Besselievre, S. Piguel, Angew. Chem., Int. $E d .2009,48,9553$. f) B. P. Berciano, S. Lebrequier, F. Besselievre, S. Piguel, S Org. Lett. 2010, 12, 4038. g) S. H. Kim, S. Chang, Org. Lett. 2010, 12, 1868.

[12] a) M. Tobisu, Y. Ano, N. Chatani, Org. Lett. 2009, 11, 3250. b) Y. H. Gu, X. M. Wang, Tetrahedron Lett. 2009, 50, 763. c) T. de Haro, C. Nevado, J. Am. Chem. Soc. 2010, 132, 1512. d) L. Yang, L. A. Zhao, C. J. Li, Chem. Commun. 2010 46,4184

[13] (a) J. P. Brand, J. Charpentier, J. Waser, Angew. Chem., Int. Ed. 2009, 48, 9346 (b) J. P. Brand, J. Waser, Angew. Chem., Int. Ed. 2010, 49, 7304. (c) J. P. Brand C. Chevalley, J. Waser, Beilstein J. Org. Chem. 2011, 7, 565. (d) J. P. Brand, , J. Waser, Org. Lett. 2012, 14, DOI:10.1021/ol203289v.

[14] Y. Wei, H. Q. Zhao, J. Kan, W. P. Su, M. C. Hong, J. Am. Chem. Soc. 2010, 132, 2522

[15] Review on alkynyl iodonium salts: a) V. V. Zhdankin, P. J. Stang, Tetrahedron 1998, 54, 10927. General hypervalent iodine chemistry: b) T. Wirth, Hypervalent iodine chemistry: modern developments in organic synthesis, Vol. 224, SPRINGER, New York, 2003. (c) V. V. Zhdankin, P. J. Stang, Chem. Rev. 2008, 108, 5299 .

[16] Other applications of alkynyl benziodoxolones: a) S. Nicolai, S. Erard, D. Fernandez Gonzalez, J. Waser, Org. Lett. 2010, 12, 384. b) S. Nicolai, C. Piemontesi, J. Waser, Angew. Chem., Int. Ed. 2011, 50, 4680. c) D. Fernandez Gonzalez, J. P. Brand, J. Waser, Chem. Eur. J. 2010, 16, 9457. d) J. P. Brand, D. Fernandez Gonzalez, S. Nicolai, J. Waser, Chem. Commun. 2011, 47, 102.

[17] First synthesis of TIPS-EBX (1): V. V. Zhdankin, C. J. Kuehl, A. P. Krasutsky, J. T. Bolz, A. J. Simonsen, J. Org. Chem. 1996, 61, 6547.
[18] Shortly after our report, Nevado reported another gold catalyzed alkynylation using hypervalent iodine reagents. See reference $12 \mathrm{c}$

[19] Y. Ohta, Y. Tokimizu, S. Oishi, N. Fujii, H. Ohno, Org. Lett. 2010, 12, 3963

[20] M. Ochiai, Y. Masaki, M. Shiro, J. Org. Chem. 1991, 56, 5511.

[21] Using a reported one-pot TIPS deprotection and Sonogashira coupling 3-((4methoxyphenyl)ethynyl)-2-phenyl- $1 H$-indole and 2-hexyl-5-((4methoxyphenyl)ethynyl)thiophene were obtained in respectively 81 and 63\% yield. See supporting information. J. W. Sun, M. P. Conley, L. M. Zhang, S. A. Kozmin, J. Am. Chem. Soc. 2006, 128, 9705.

[22] a) I. Kieltsch, P. Eisenberger, A. Togni, Angew. Chem., Int. Ed. 2007, 46, 754 b) P. Eisenberger, I. Kieltsch, N. Armanino, A. Togni, Chem. Commun. 2008 1575. c) K. Stanek, R. Koller, A. Togni, J. Org. Chem. 2008, 73, 7678. d) R. Koller, K. Stanek, D. Stolz, R. Aardoom, K. Niedermann, A. Togni, Angew. Chem. Int. Edit. 2009, 48, 4332. e) M. S. Wiehn, E. V. Vinogradova, A. Togni, J. Fluor. Chem. 2010, 131, 951. f) K. Niedermann, J. M. Welch, R. Koller, J. Cvengros, N. Santschi, P. Battaglia, A. Togni, Tetrahedron 2010, 66, 5753. g) K. Niedermann, N. Fruh, E. Vinogradova, M. S. Wiehn, A. Moreno, A. Togni, Angew. Chem., Int. Ed. 2011, 50, 1059. See also: h) A. E. Allen, D. W. C MacMillan, J. Am. Chem. Soc. 2010, 132, 4986.

[23] M. Ochiai, T. Sueda, K. Miyamoto, P. Kiprof, V. V. Zhdankin, Angew. Chem. Int. Ed. 2006, 45, 8203.

[24] a) A. S. K. Hashmi, Chem. Rev. 2007, 107, 3180. b) D. J. Gorin, B. D. Sherry, F D. Toste, Chem. Rev. 2008, 108, 3351. c) A. Fürstner, P. W. Davies, Angew. Chem., Int. Ed. 2007, 46, 3410. d) Z. G. Li, C. Brouwer, C. He, Chem. Rev. 2008, 108, 3239 .

[25] a) H. A. Wegner, S. Ahles, M. Neuburger, Chem. Eur. J. 2008, 14, 11310. b) A S. K. Hashmi, T. D. Ramamurthi, F. Rominger, J. Organomet. Chem. 2009, 694 592. c) G. Z. Zhang, Y. Peng, L. Cui, L. M. Zhang, Angew. Chem., Int. Ed. 2009, 48, 3112. d) M. N. Hopkinson, A. D. Gee, V. Gouverneur, Chem. Eur. J. 2011, 17, 8248. e) P. Garcia, M. Malacria, C. Aubert, V. Gandon, L. Fensterbank, ChemCatChem 2010, 2, 493. f) H. A. Wegner, M. Auzias, Angew. Chem., Int Ed. 2011, 50, 3236.

[26] a) Y. Kita, H. Tohma, M. Inagaki, K. Hatanaka, T. Yakura, Tetrahedron Lett. 1991, 32, 4321. b) Y. Kita, H. Tohma, K. Hatanaka, T. Takada, S. Fujita, S Mitoh, H. Sakurai, S. Oka, J. Am. Chem. Soc. 1994, 116, 3684. c) T. Dohi, M. Ito, N. Yamaoka, K. Morimoto, H. Fujioka, Y. Kita, Tetrahedron 2009, 65, 10797. d) K. Morimoto, N. Yamaoka, C. Ogawa, T. Nakae, H. Fujioka, T. Dohi, Y. Kita, Org. Lett. 2010, 12, 3804. e) T. Dohi, M. Ito, N. Yamaoka, K Morimoto, H. Fujioka, Y. Kita, Angew. Chem., Int. Ed. 2010, 49, 3334.

[27] a) S.Oae, Y. Uchida, Acc. Chem. Res. 1991, 24, 202. b) M. Ochiai, Top. Curr Chem. 2003, 224, 5-68.

[28] M. Ochiai, M. Kunishima, Y. Nagao, K. Fuji, M. Shiro, E. Fujita, J. Am. Chem Soc. 1986, 108, 8281 .

[29] For proposed oxidative addition of hypervalent iodine reagents on other metals, see: a) N. R. Deprez, M. S. Sanford, Inorg. Chem. 2007, 46, 1924. b) R. J. Phipps, N. P. Grimster, M. J. Gaunt, J. Am. Chem. Soc. 2008, 130, 8172. c) R. J. Phipps, M. J. Gaunt, Science 2009, 323, 1593. d) A. E. Allen, D. W. C. MacMillan, J. Am. Chem. Soc. 2011, 133, 4260.

[30] Review on C-H bond auration: a) T. C. Boorman, I. Larrosa, Chem. Soc. Rev. 2011, 40, 1910. Selected examples: b) M. S. Kharasch, H. S. Isbell, J. Am. Chem Soc. 1931, 53, 3053. c) Y. Fuchita, Y.Utsunomiya, M. Yasutake, J. Chem. Soc. Dalton Trans. 2001, 2330. d) M. T. Reetz, K. Sommer, Eur. J. Org. Chem. 2003, 3485. e) Z. G. Li, D. A. Capretto, R. O. Rahaman, C. He, J. Am. Chem. Soc. 2007, 129, 12058. f) P. F. Lu, T. C. Boorman, A. M. Z. Slawin, I. Larrosa, J. Am Chem. Soc. 2010, 132, 5580.

[31] Chloro[tris(2,4-di-tert-butylphenyl)phosphite]gold and (Acetonitrile)[(2biphenyl)di-tert-butylphosphine]gold(I) hexafluoroantimonate did not afford any product.

[32] a) L. P. Liu, B. Xu, M. S. Mashuta, G. B. Hammond, J. Am. Chem. Soc. 2008 , 130, 17642. b) L. P. Liu, G. B. Hammond, Chem. Asian J. 2009, 4, 1230. c) A. S. K. Hashmi, A. M. Schuster, F. Rominger, Angew. Chem., Int. Ed. 2009, 48, 8247. d) A. S. K. Hashmi, Gold Bull. 2009, 42, 275. e) D. Weber, M. A. Tarselli, M. R. Gagne, Angew. Chem., Int. Ed. 2009, 48, 5733. f) X. M. Zeng, R. Kinjo, B. Donnadieu, G. Bertrand, Angew. Chem., Int. Ed. 2010, 49, 942.

[33] In our primary screening $\mathrm{AuCl}_{3}$ afforded 56\% product. For details see ref 13a.

[34] For metal free arylation using hypervalent iodine reagents, see: a) L. Ackermann, M. Dell'Acqua, S. Fenner, R. Vicente, R. Sandmann, Org. Lett. 2011, 13, 2358 b) C. L. Ciana, R. J. Phipps, J. R. Brandt, F. M. Meyer, M. J. Gaunt, Ang. Chem. 
Int. Ed. 2011, 50, 458. Using the conditions reported by Ackermann, no product was detected.

[35] Using Mayr scale: S. Lakhdar, M. Westermaier, F. Terrier, R. Goumont, T Boubaker, A. R. Ofial, H. Mayr, J. Org. Chem. 2006, 71, 9088. N: nucleophilicity parameter.

[36] See supporting information.

[37] For other examples of rate acceleration based on ortho substitution, see: a) J. T. Su, W. A. Goddard, J. Am. Chem. Soc. 2005, 127, 14146. b) M. Uyanik, M. Akakura, K. Ishihara, J. Am. Chem. Soc. 2008, 131, 251. c) J. N. Moorthy, K. Senapati, K. N. Parida, S. Jhulki, K. Sooraj, N. N. Nair, J. Org. Chem. 2011, 76 , 9593. d) A. A. Guilbault, C. Y. Legault, ACS Catalysis 2012, 219

[38] A. S. K. Hashmi, M. C. Blanco, D. Fischer, J. W. Bats, Eur. J. Org. Chem. 2006 1387.

[39] Commercially available solvent was used without drying or purification. 
Received: ((will be filled in by the editorial staff))

Revised: ((will be filled in by the editorial staff))

Published online: ((will be filled in by the editorial staff)) 
Entry for the Table of Contents (Please choose one layout only)

Layout 1:

Catch Phrase

Author(s), Corresponding

Author $(s) *$............ Page - Page

Title Text

Layout 2:

\section{Direct}

functionalization-

Jonathan P. Brand, Clara

Chevalley, Rosario Scopelliti and

Jérôme Waser *............ Page -

Page

Ethynyl BenziodoXolones for the

Direct Alkynylation of

Heterocycles: Structural

Requirement, Improved

Procedure for Pyrroles and

Insights into the Mechanism.
Text for Table of Contents, max. 450 characters.

((The TOC Graphic should not exceed the size of this area))

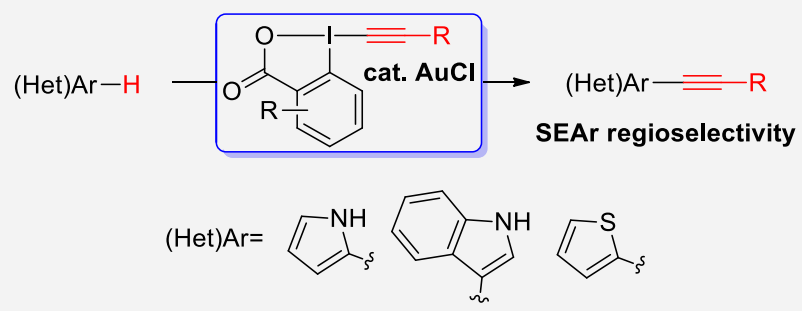

"Hyperviolent" iodine: Further insight into the direct alkynylation of electron-rich heterocycles using Ethynyl BenziodoXolone (EBX) reagents is given. An improved procedure for pyrroles, an in depth structure-reactivity study of the hypervalent iodine reagents and mechanism investigations are presented to shade light on this unique alkynylation method. 


\title{
Supporting Information
}

\author{
67 pages
}

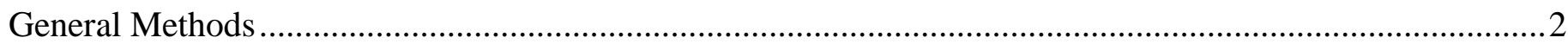

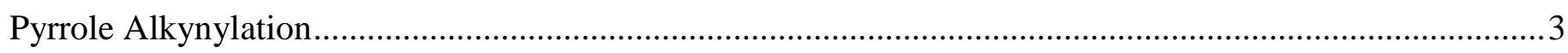

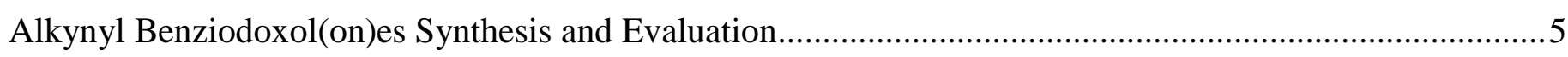

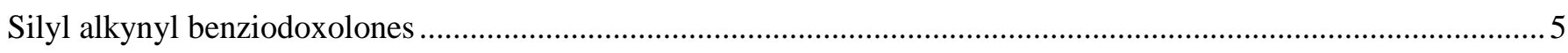

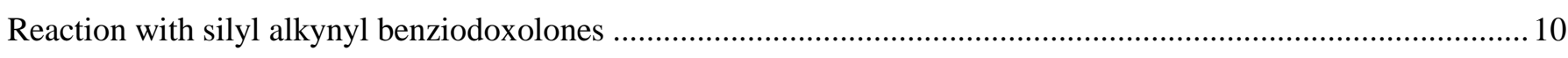

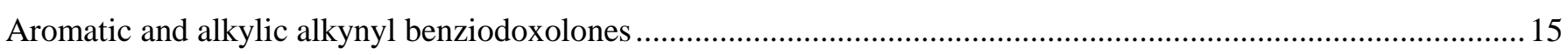

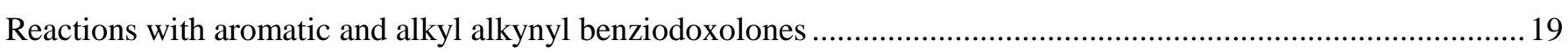

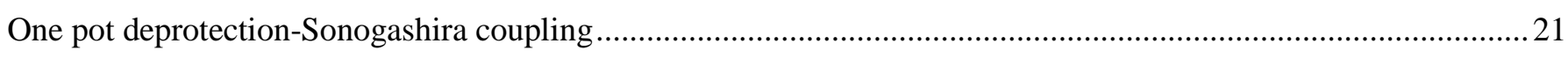

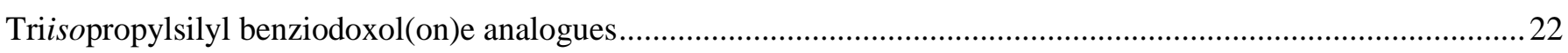

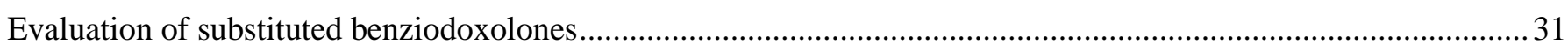

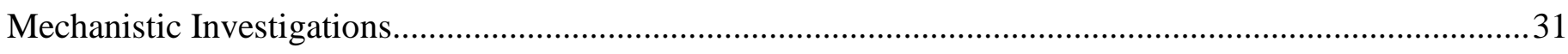

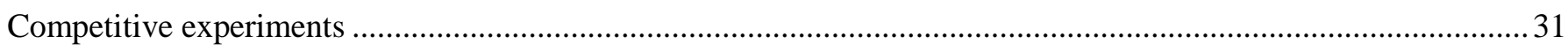

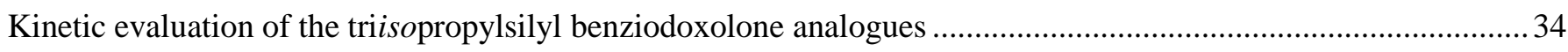

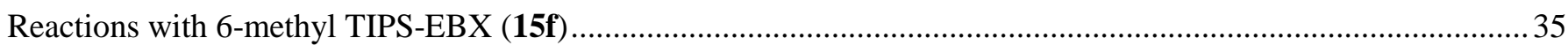

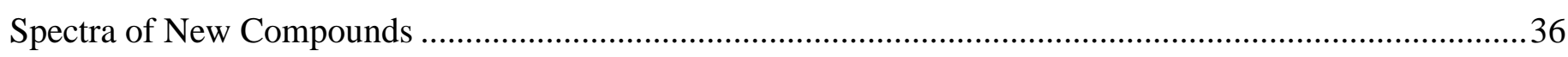




\section{General Methods}

All reactions were carried out in oven dried glassware under an atmosphere of nitrogen, unless stated otherwise. For quantitative flash chromatography technical grade solvents were used. For flash chromatography for analysis, HPLC grade solvents from Sigma-Aldrich were used. THF, $\mathrm{Et}_{2} \mathrm{O}, \mathrm{CH}_{3} \mathrm{CN}_{\text {, }}$ toluene, hexane and $\mathrm{CH}_{2} \mathrm{Cl}_{2}$ were dried by passage over activated alumina under nitrogen atmosphere $\left(\mathrm{H}_{2} \mathrm{O}\right.$ content $<10 \mathrm{ppm}$, Karl-Fischer titration). $\mathrm{NEt}_{3}$ and pyridine were distilled under nitrogen from KOH. Gold chloride was purchased from Aldrich or Alfa Aesar and kept in desiccator under anhydrous condition (decrease of reactivity has been observed for catalyst if prolonged exposition to air ( $\mathrm{ca} 1 \mathrm{month}$ ). All chemicals were purchased from Acros, Aldrich, Fluka, VWR, Aplichem or Merck and used as such unless stated otherwise. Chromatographic purification was performed as flash chromatography using MachereyNagel silica 40-63, $60 \AA$, using the solvents indicated as eluent with 0.1-0.5 bar pressure. TLC was performed on Merck silica gel $60 \mathrm{~F}_{254}$ TLC glass plates or aluminium plates and visualized with UV light, permanganate stain, CAN stain or anisaldehyde stain. Melting points were measured on a calibrated Büchi B-540 melting point apparatus using open glass capillaries. ${ }^{1} \mathrm{H}-\mathrm{NMR}$ spectra were recorded on a Brucker DPX-400 $400 \mathrm{MHz}$ spectrometer in chloroform-d, $\mathrm{C}_{6} \mathrm{D}_{6}$ DMSO- $\mathrm{d}_{6}$ or $\mathrm{CD}_{3} \mathrm{OD}$, all signals are reported in ppm with the internal chloroform signal at $7.26 \mathrm{ppm}$, the internal $\mathrm{C}_{6} \mathrm{D}_{6}$ signal at $7.16 \mathrm{ppm}$. the internal DMSO signal at $2.50 \mathrm{ppm}$ or the internal methanol signal at $3.30 \mathrm{ppm}$ as standard. The data is being reported as $(\mathrm{s}=$ singlet, $\mathrm{d}=$ doublet, $\mathrm{t}=$ triplet, $\mathrm{q}=$ quadruplet, $\mathrm{qi}=$ quintet, $\mathrm{m}=$ multiplet or unresolved, br = broad signal, app = apparent, coupling constant(s) in Hz, integration, interpretation). ${ }^{13} \mathrm{C}$ NMR spectra were recorded with ${ }^{1} \mathrm{H}$-decoupling on a Brucker DPX-400 $100 \mathrm{MHz}$ spectrometer in chloroform-d, DMSO- $\mathrm{d}_{6}$ or $\mathrm{CD}_{3} \mathrm{OD}$, all signals are reported in ppm with the internal chloroform signal at $77.0 \mathrm{ppm}$, the internal DMSO signal at $39.5 \mathrm{ppm}$ or the internal methanol signal at $49.0 \mathrm{ppm}$ as standard. Some benziodoxol(on)es NMRs in $\mathrm{CDCl}_{3}$ showed a small dependence to the concentration. As a result, the concentration of the corresponding NMR samples is indicated. Infrared spectra were recorded on a JASCO FT-IR B4100 spectrophotometer with an ATR PRO410-S and a ZnSe prisma and are reported as $\mathrm{cm}^{-1}(\mathrm{w}=$ weak, $\mathrm{m}=$ medium, $\mathrm{s}=$ strong, $\mathrm{br}=$ broad). Gas chromatographic and low resolution mass spectrometric measurements were performed on a Perkin-Elmer Clarus 600 gas chromatographer and mass spectrometer using a Perkin-Elemer Elite fused silica column (length: $30 \mathrm{~m}$, diameter: $0.32 \mathrm{~mm}$ ) and Helium as carrier gas. High resolution mass spectrometric measurements were performed by the mass spectrometry service of ISIC at the EPFL on a MICROMASS (ESI) Q-TOF Ultima API. HPLC measurement were done on a JASCO HPLC system with an AS2055 Autosampler, a PU 2089 Pump, a UV 2075 detector and a SEDEX 85 (SEDERE) detector using a CHIRALPAK IC column from DAICEL Chemical Industries Ltd. HPLC grade solvents from Sigma-Aldrich were used. 


\section{Pyrrole Alkynylation}

\section{General procedure of pyrrole alkynylation using pyridine:}

$\mathrm{AuCl}$ (2.3 mg, $0.010 \mathrm{mmol}, 0.05$ equiv) was added to a stirring solution of pyridine ( $19 \mu \mathrm{L}, 0.024 \mathrm{mmol}, 1.2$ equiv), TIPS-EBX (1) (103 mg, $0.240 \mathrm{mmol}, 1.2$ equiv) and the corresponding pyrrole (0.200 mmol, 1.0 equiv) in $\mathrm{Et}_{2} \mathrm{O}^{1}(4 \mathrm{~mL})$ under air. The reaction was sealed and stirred at room temperature for $15 \mathrm{~h}$. $\mathrm{Et}_{2} \mathrm{O}(10$ $\mathrm{mL}$ ) was added, the organic layer was washed twice with $0.1 \mathrm{M} \mathrm{NaOH}(15 \mathrm{~mL})$. The aqueous layers were combined and extracted with $\mathrm{Et}_{2} \mathrm{O}(20 \mathrm{~mL})$. The organic layers were combined, washed with saturated $\mathrm{NaHCO}_{3}(20 \mathrm{~mL})$, brine $(20 \mathrm{~mL})$, dried with $\mathrm{MgSO}_{4}$ and concentrated under reduced pressure.

1-Methyl-2-((triisopropylsilyl)ethynyl)-1H-pyrrole (3a), 1-Methyl-3-((triisopropylsilyl)ethynyl)-1H-pyrrole (3a')

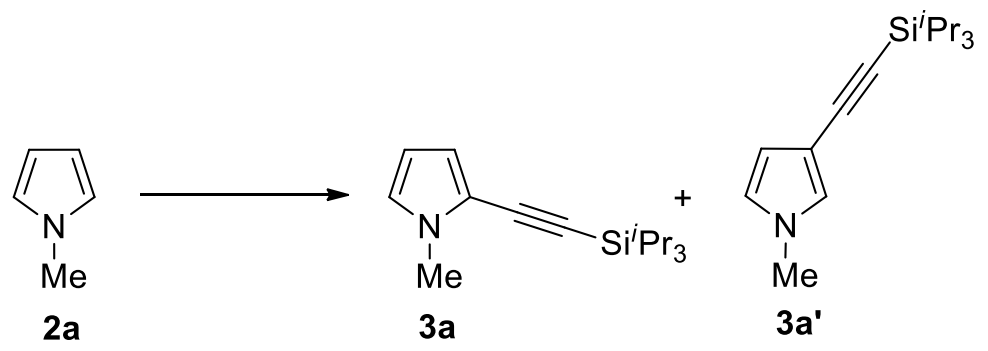

Following the general procedure but using $\mathbf{2 a}(53 \mu \mathrm{L}, 0.60 \mathrm{mmol}, 3$ equiv) and 1 ( $85 \mathrm{mg}, 0.20 \mathrm{mmol}, 1$ equiv). Purification by flash chromatography (PET) afforded $\mathbf{3 a}(37 \mathrm{mg}, 0.14 \mathrm{mmol}, 71 \%)$ as a colorless oil and 3a' $(9.0 \mathrm{mg}, 0.034 \mathrm{mmol}, 17 \%)$ as yellow oil. 3a: $\mathrm{R}_{f}(\mathrm{PET}): 0.1 .{ }^{1} \mathrm{H} \mathrm{NMR}\left(\mathrm{CDCl}_{3}, 400 \mathrm{MHz}\right) \delta 6.62(\mathrm{dd}$, $J=2.6,1.9 \mathrm{~Hz}, 1 \mathrm{H}, \mathrm{ArH}), 6.44(\mathrm{~m}, 1 \mathrm{H}, \mathrm{ArH}), 6.06(\mathrm{~m}, 1 \mathrm{H}, \mathrm{ArH}), 3.69\left(\mathrm{~d}, J=0.3 \mathrm{~Hz}, 3 \mathrm{H}, \mathrm{CH}_{3}\right), 1.14(\mathrm{~m}$, $21 \mathrm{H}, \mathrm{TIPS}) .{ }^{13} \mathrm{C} \mathrm{NMR}\left(\mathrm{CDCl}_{3}, 100 \mathrm{MHz}\right) \delta 125.5,123.4,115.0,107.8,98.4,94.5,34.6,18.7,11.4 .3 \mathrm{a}$ ': $\mathrm{R} f$ (PET): 0.4. ${ }^{1} \mathrm{H} \mathrm{NMR}\left(\mathrm{CDCl}_{3}, 400 \mathrm{MHz}\right) \delta 6.83(\mathrm{~m}, 1 \mathrm{H}, \mathrm{ArH}), 6.47(\mathrm{t}, J=2.2 \mathrm{~Hz}, 1 \mathrm{H}, \mathrm{ArH}), 6.24(\mathrm{~m}, 1 \mathrm{H}$, $\mathrm{ArH}), 3.61$ (s, $\left.3 \mathrm{H}, \mathrm{CH}_{3}\right), 1.10$ (m, $\left.21 \mathrm{H}, \mathrm{TIPS}\right) .{ }^{13} \mathrm{C} \mathrm{NMR}\left(\mathrm{CDCl}_{3}, 100 \mathrm{MHz}\right) \delta 126.3,121.5,112.5,104.8$, 102.6, 87.5, 36.3, 18.7, 11.5. In accordance with reported data. ${ }^{2}$

2-Ethyl-5-((triiso-propylsilyl)ethynyl)-1H-pyrrole (3b)

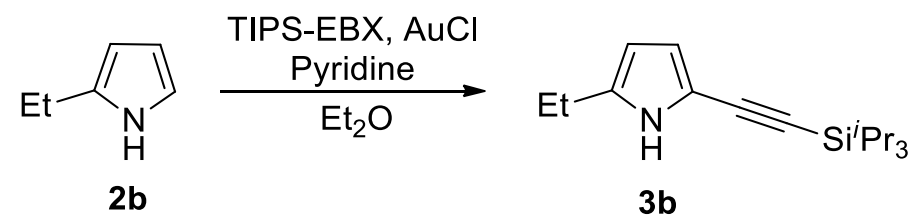

Purification by flash chromatography (PET to PET/ $\mathrm{CH}_{2} \mathrm{Cl}_{2}$ 99/1) afforded $3 \mathbf{b}$ (45 mg, 0.16 mmol, $81 \%$ ) as colorless oil. $\mathrm{R}_{f}\left(\mathrm{PET} / \mathrm{CH}_{2} \mathrm{Cl}_{2}\right.$ 99/1): 0.1. ${ }^{1} \mathrm{H} \mathrm{NMR}\left(\mathrm{CDCl}_{3}, 400 \mathrm{MHz}\right) \delta 8.04$ (br s, $\left.1 \mathrm{H}, \mathrm{NH}\right), 6.40$ (dd, $J=$ 3.4, $2.9 \mathrm{~Hz}, 1 \mathrm{H}, \mathrm{ArH}), 5.86(\mathrm{~m}, 1 \mathrm{H}, \mathrm{ArH}), 2.62$ (q, $\left.J=7.7 \mathrm{~Hz}, 2 \mathrm{H}, \mathrm{CH}_{2}\right), 1.25$ (t, J= 7.6 Hz, $3 \mathrm{H}, \mathrm{CH}_{3}$ ), $1.12(\mathrm{~m}, 21 \mathrm{H}, \mathrm{TIPS}) .{ }^{13} \mathrm{C} \mathrm{NMR}\left(\mathrm{CDCl}_{3}, 100 \mathrm{MHz}\right) \delta 135.9,115.8,111.7,105.5,99.5,91.3,21.0,18.7,13.5$, 11.4. In accordance with reported data. ${ }^{2}$

\section{2-Phenyl-5-((triiso-propylsilyl)ethynyl)-1H-pyrrole (3c)}

\footnotetext{
${ }^{1}$ Commercially available solvent was used without drying or purification.

${ }^{2}$ Brand, J. P.; Charpentier, J.; Waser, J. Angew. Chem., Int. Ed. 2009, 48, 9346.
} 


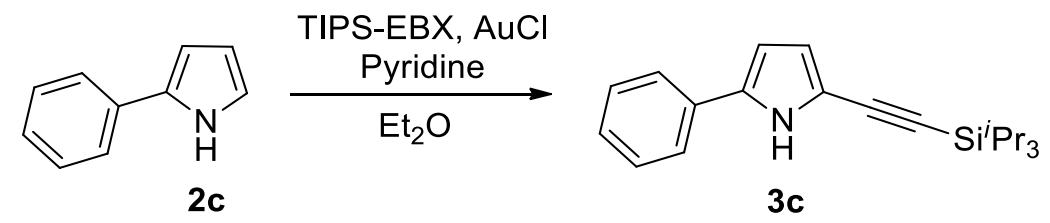

On 0.49 mmol. Purification by flash chromatography (PET/ $\mathrm{CH}_{2} \mathrm{Cl}_{2}$ 95/5) afforded 3c $(133 \mathrm{mg}, 0.411$ mmol, $84 \%)$ as amorphous solid. $\mathrm{R}_{f}\left(\mathrm{PET}_{\mathrm{Et}} \mathrm{O} 5 / 95\right)$ : 0.2. ${ }^{1} \mathrm{H} \mathrm{NMR}\left(\mathrm{CDCl}_{3}, 400 \mathrm{MHz}\right) \delta 8.54$ ( br s, $1 \mathrm{H}$, $\mathrm{NH}), 7.52$ (m, $2 \mathrm{H}, \mathrm{ArH}), 7.41$ (m, $2 \mathrm{H}, \mathrm{ArH}), 7.28$ (m, $1 \mathrm{H}, \mathrm{ArH}), 6.60$ (dd, J= 2.2, $3.4 \mathrm{~Hz}, 1 \mathrm{H}, \mathrm{ArH}), 6.50$ $(\mathrm{dd}, J=2.7,3.6 \mathrm{~Hz}, 1 \mathrm{H}, \mathrm{ArH}), 1.19$ (m, $21 \mathrm{H}, \mathrm{TIPS}) .{ }^{13} \mathrm{C} \mathrm{NMR}\left(\mathrm{CDCl}_{3}, 100 \mathrm{MHz}\right) \delta 133.1,131.9,129.0$, $126.9,124.2,117.3,114.3,106.8,99.0,93.1,18.8,11.4$. In accordance with reported data. ${ }^{2}$

\section{3,5-dimethyl-2-((triisopropylsilyl)ethynyl)-1H-pyrrole (3d) and 2,4-dimethyl-3,5- bis((triisopropylsilyl)ethynyl)-1H-pyrrole (31)}

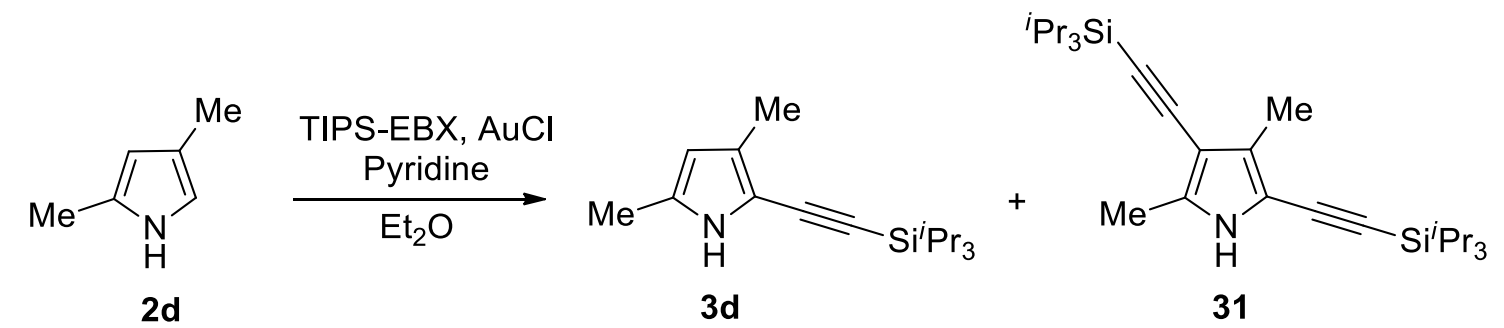

Purification by flash chromatography (PET/ $\mathrm{CH}_{2} \mathrm{Cl}_{2}$ 95/5) afforded $\mathbf{3 d}(31 \mathrm{mg}, 0.11 \mathrm{mmol}, 56 \%)$ as colorless oil and $\mathbf{3 1}(14 \mathrm{mg}, 0.031 \mathrm{mmol}, 15 \%)$ as colorless oil.

With 3 equivalent of TIPS-EBX (1) $(258 \mathrm{mg}, 0.600 \mathrm{mmol})$ purification by flash chromatography (PET/ $\mathrm{CH}_{2} \mathrm{Cl}_{2}$ 95/5) afforded 31 (66 $\mathrm{mg}, 0.15 \mathrm{mmol}, 73 \%$ ) as colorless oil.

3d: $\mathrm{R}_{f}\left(\mathrm{PET} / \mathrm{CH}_{2} \mathrm{Cl}_{2}\right.$ 95/5): 0.2. ${ }^{1} \mathrm{H} \mathrm{NMR}\left(400 \mathrm{MHz}, \mathrm{CDCl}_{3}\right) \delta 7.80$ (br s, $\left.1 \mathrm{H}, \mathrm{NH}\right), 5.70$ (s, $\left.1 \mathrm{H}, \mathrm{ArH}\right)$, 2.20 (s, $\left.3 \mathrm{H}, \mathrm{CH}_{3}\right), 2.12\left(\mathrm{~m}, 3 \mathrm{H}, \mathrm{CH}_{3}\right), 1.11$ (m, $\left.21 \mathrm{H}, \mathrm{TIPS}\right) .{ }^{13} \mathrm{C} \mathrm{NMR}\left(101 \mathrm{MHz}, \mathrm{CDCl}_{3}\right) \delta 128.7,127.0$, 110.1, 108.3, 99.0, 93.7, 18.7, 13.1, 11.7, 11.3. IR 3477 (w), 3377 (w), 2941 (s), 2924 (m), 2864 (s), 2137 (s), $1718(w), 1583(\mathrm{w}), 1493(\mathrm{w}), 1451(\mathrm{w}), 1382(\mathrm{w}), 1351(\mathrm{w}), 1299(\mathrm{w}), 1245(\mathrm{w}), 1146(\mathrm{w}), 1073(\mathrm{w})$, 1017 (w), $996(\mathrm{~m}), 919$ (w), $883(\mathrm{~m}), 838$ (w), $812(\mathrm{~m}), 791(\mathrm{w}), 701$ (m), 677 (s), 650 (m). HRMS (ESI) calcd for $\mathrm{C}_{17} \mathrm{H}_{30} \mathrm{NSi}^{+}[\mathrm{M}+\mathrm{H}]^{+}$276.2142; found 276.2135. 31: $\mathrm{R} f 0.1\left(\mathrm{PET} / \mathrm{CH}_{2} \mathrm{Cl}_{2}\right.$ 95/5). ${ }^{1} \mathrm{H}$ NMR (400 $\mathrm{MHz}_{\mathrm{CDCl}}$ ) $\delta 7.84$ (br s, $\left.1 \mathrm{H}, \mathrm{NH}\right), 2.29$ (s, $\left.3 \mathrm{H}, \mathrm{Me}\right), 2.15$ (m, $\left.3 \mathrm{H}, \mathrm{Me}\right), 1.12$ (m, 42 H, TIPS). ${ }^{13} \mathrm{C}$ NMR $\left(101 \mathrm{MHz}, \mathrm{CDCl}_{3}\right) \delta 133.3,128.9,109.5,104.7,101.0,98.1,94.1,92.0,18.7,18.7,12.3,11.3,11.3,10.8$. IR 3472 (w), 3377 (w), 2942 (s), 2865 (s), 2140 (s), 1716 (w), 1579 (w), 1383 (w), 1298 (w), 1206 (w), 1132 (w), 1073 (w), 1017 (w), 996 (w), 889 (w), 865 (w), 812 (w), 780 (w), 713 (w), 674 (s). HRMS (ESI) calcd for $\mathrm{C}_{28} \mathrm{H}_{50} \mathrm{NSi}_{2}{ }^{+}[\mathrm{M}+\mathrm{H}]^{+} 456.3476$; found 456.3491 .

2,5-Dimethyl-3-((triiso-propylsilyl)ethynyl)-1H-pyrrole (3e)

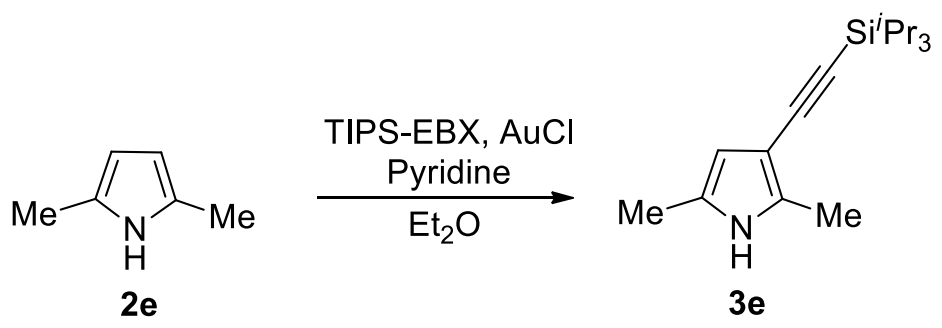


Purification by flash chromatography (PET/Et $2 \mathrm{O} 85 / 15)$ afforded $3 \mathbf{e}(40 \mathrm{mg}, 0.15 \mathrm{mmol}, 73 \%)$ as yellow oil. $\mathrm{R}_{f}\left(\mathrm{PET} / \mathrm{Et}_{2} \mathrm{O} 85: 15\right): 0.3 .{ }^{1} \mathrm{H} \mathrm{NMR}\left(\mathrm{CDCl}_{3}, 400 \mathrm{MHz}\right) \delta 7.58(\mathrm{br} \mathrm{s}, 1 \mathrm{H}, \mathrm{NH}), 5.90(\mathrm{~d}, J=1.7 \mathrm{~Hz}, 1 \mathrm{H}$, $\mathrm{ArH}$ ), 2.32 (s, $3 \mathrm{H}, \mathrm{Me}$ ), 2.20 (s, $3 \mathrm{H}, \mathrm{Me}), 1.14$ (m, $21 \mathrm{H}, \mathrm{TIPS}) .{ }^{13} \mathrm{C} \mathrm{NMR}\left(\mathrm{CDCl}_{3}, 100 \mathrm{MHz}\right) \delta 132.3$, $125.5,108.8,103.1,102.3,89.1,18.8,12.8,12.0,11.5$. In accordance with reported data. ${ }^{2}$

\section{1,2,5-Trimethyl-3-((triisopropylsilyl)ethynyl)- $1 H$-pyrrole (3f)}

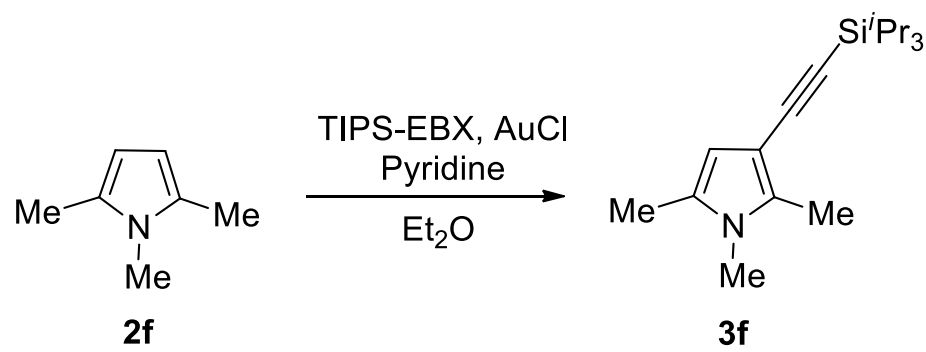

Purification by flash chromatography (PET/ $\mathrm{CH}_{2} \mathrm{Cl}_{2}$ 95/5) afforded $\mathbf{3 f}(56 \mathrm{mg}, 0.19 \mathrm{mmol}, 97 \%$ ) as colorless oil. Rf 0.3 (PET/ $\mathrm{CH}_{2} \mathrm{Cl}_{2}$ 95/5). ${ }^{1} \mathrm{H}$ NMR (400 MHz, $\left.\mathrm{CDCl}_{3}\right) \delta 5.92(\mathrm{~s}, 1 \mathrm{H}, \mathrm{ArH}), 3.36(\mathrm{~s}, 3 \mathrm{H}$, $\mathrm{CH}_{3}$ ), 2.29 (s, $\left.3 \mathrm{H}, \mathrm{CH}_{3}\right), 2.16$ (s, $\left.3 \mathrm{H}, \mathrm{CH}_{3}\right), 1.12$ (m, $\left.21 \mathrm{H}, \mathrm{TIPS}\right) .{ }^{13} \mathrm{C} \mathrm{NMR}\left(101 \mathrm{MHz}, \mathrm{CDCl}_{3}\right) \delta 133.7$, 127.3, 108.0, 103.4, 101.0, 88.7, 30.5, 18.7, 12.2, 11.4, 11.3. IR 2957 (m), 2939 (m), $2863(\mathrm{~m}), 1533(\mathrm{w})$, 1462 (m), 1439 (w), 1417 (w), 1389 (w), 1349 (m), 1243 (w), 1190 (w), 1101 (w), 1074 (w), 1015 (w), 999 (m), 918 (w), 882 (s), 839 (w), 769 (m), 735 (w), 701 (s), 657 (s). HRMS (ESI) calcd for $\mathrm{C}_{18} \mathrm{H}_{32} \mathrm{NSi}^{+}$ $[\mathrm{M}+\mathrm{H}]^{+}$290.2299; found 290.2298.

3-Ethyl-2,4-dimethyl-5-((triiso-propylsilyl)ethynyl)-1H-pyrrole (3g)<smiles>CCc1c(C)c[nH]c1C</smiles>

$2 \mathrm{~g}$

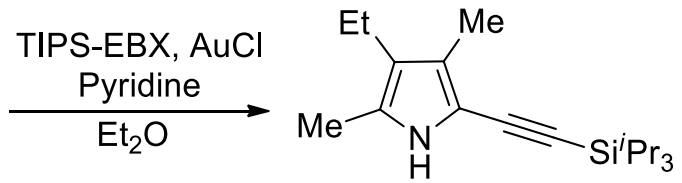

$3 \mathbf{g}$

Purification by flash chromatography ( $\mathrm{PET} / \mathrm{CH}_{2} \mathrm{Cl}_{2}$ 99/1) afforded $3 \mathrm{~g}(50 \mathrm{mg}, 0.17 \mathrm{mmol}, 83 \%)$ as yellow oil. $\mathrm{R}_{f}\left(\mathrm{PET} / \mathrm{CH}_{2} \mathrm{Cl}_{2}\right.$ 99/1): 0.25. ${ }^{1} \mathrm{H} \mathrm{NMR}\left(\mathrm{CDCl}_{3}, 400 \mathrm{MHz}\right) \delta 7.74$ (br s, $\left.1 \mathrm{H}, \mathrm{NH}\right), 2.40$ (q, $J=7.6$ $\mathrm{Hz}, 2 \mathrm{H}, \mathrm{CH}_{2}$ ), 2.19 (s, $3 \mathrm{H}, \mathrm{Me}$ ), 2.14 (s, $3 \mathrm{H}, \mathrm{Me}$ ), 1.15 (m, $\left.21 \mathrm{H}, \mathrm{TIPS}\right), 1.08$ (t, J=7.7 Hz, $3 \mathrm{H}, \mathrm{CH}_{2} \mathrm{CH}_{3}$ ). ${ }^{13} \mathrm{C}$ NMR $\left(\mathrm{CDCl}_{3}, 100 \mathrm{MHz}\right) \delta 125.5,124.7,121.4,108.9,99.4,93.6,18.8,17.6,15.4,11.4,11.2,10.0$. In accordance with reported data. ${ }^{2}$

\section{Alkynyl Benziodoxol(on)es Synthesis and Evaluation}

\section{Silyl alkynyl benziodoxolones}

Triisopropylsilyl trimethylsilylacetylene (14)

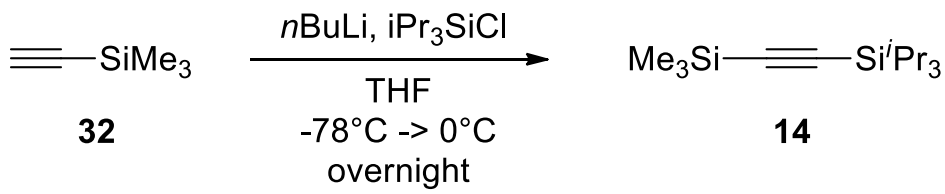


Following a reported procedure, ${ }^{3} n$-butyllithium $(2.5 \mathrm{M}$ in hexanes, $12.0 \mathrm{~mL}, 29.9 \mathrm{mmol}, 0.98$ equiv) was added dropwise to a stirred solution of ethynyltrimethylsilane (32) (3.0 g, $30 \mathrm{mmol}, 1.0$ equiv) in THF (48 $\mathrm{mL}$ ) at $-78{ }^{\circ} \mathrm{C}$. The mixture was then warmed to $0{ }^{\circ} \mathrm{C}$ and stirred for $5 \mathrm{~min}$. The mixture was then cooled back to $-78{ }^{\circ} \mathrm{C}$ and chlorotriisopropylsilane $(6.4 \mathrm{~mL}, 30 \mathrm{mmol}, 1.0$ equiv) was added dropwise. The mixture was then allowed to warm to room temperature and stirred overnight. A saturated solution of ammonium chloride $(40 \mathrm{~mL})$ was added, and the reaction mixture was extracted with diethyl ether $(2 \times 60 \mathrm{~mL})$. The organic layer was washed with water and brine, then dried over $\mathrm{MgSO}_{4}$, filtered and concentrated under reduced pressure to obtain a colorless liquid which was further purified by Kugelrohr distillation (56$\left.57^{\circ} \mathrm{C} / 0.25 \mathrm{mmHg}\right)$ to yield $14(7.16 \mathrm{~g}, 28.0 \mathrm{mmol}, 92 \%$ yield $)$ as a colorless liquid. ${ }^{1} \mathrm{H}$ NMR (400 MHz, $\left.\mathrm{CDCl}_{3}\right) \delta 1.08$ (m, $21 \mathrm{H}$, TIPS), 0.18 (s, 9 H, TMS). IR v 2959 (m), 2944 (m), 2896 (w), 2867 (m), $1464(\mathrm{w})$, $1385(\mathrm{w}), 1250(\mathrm{~m}), 996(\mathrm{w}), 842(\mathrm{~s}), 764(\mathrm{~s}), 675(\mathrm{~m}), 660(\mathrm{~m})$. Characterization data 14 corresponded to the literature values. ${ }^{3}$

\section{1-Hydroxy-1,2-benziodoxol-3(1H)-one (4)}<smiles>O=C(O)c1ccccc1I</smiles>

18

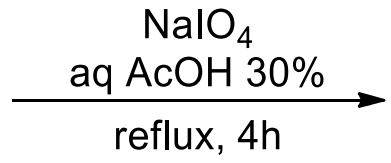

reflux, $4 \mathrm{~h}$<smiles>O=C1OI(O)c2ccccc21</smiles>

Following a reported procedure, ${ }^{4} \mathrm{NaIO}_{4}(6.7 \mathrm{~g}, 31 \mathrm{mmol} ; 1.0$ equiv) and 2-iodobenzoic acid (18) (7.4 g, $30 \mathrm{mmol}, 1.0$ equiv) were suspended in $30 \%$ (v:v) aq. $\mathrm{AcOH}(45 \mathrm{~mL})$. The mixture was vigorously stirred and refluxed for $4 \mathrm{~h}$. The reaction mixture was then diluted with cold water $(120 \mathrm{~mL})$ and allowed to cool to room temperature, protecting it from light. After $1 \mathrm{~h}$, the crude product was collected by filtration, washed on the filter with ice water $(3 \times 30 \mathrm{~mL})$ and acetone $(3 \times 30 \mathrm{~mL})$, and air-dried in the dark to give the pure product 4 (7.3 g, $19 \mathrm{mmol}, 92 \%$ yield) as a colorless solid. ${ }^{1} \mathrm{H} \mathrm{NMR}\left(400 \mathrm{MHz},\left(\mathrm{CD}_{3}\right)_{2} \mathrm{SO}\right) \delta 8.02(\mathrm{dd}, J=$ 7.7, $1.4 \mathrm{~Hz}, 1 \mathrm{H}, \operatorname{Ar} H), 7.97(\mathrm{~m}, 1 \mathrm{H}, \operatorname{ArH}), 7.85(\mathrm{dd}, J=8.2,0.7 \mathrm{~Hz}, 1 \mathrm{H}, \operatorname{Ar} H), 7.71(\mathrm{td}, J=7.6,1.2 \mathrm{~Hz}, 1$ $\mathrm{H}, \mathrm{Ar} H) .{ }^{13} \mathrm{C} \mathrm{NMR}\left(100 \mathrm{MHz},\left(\mathrm{CD}_{3}\right)_{2} \mathrm{SO}\right) \delta 167.7,134.5,131.5,131.1,130.4,126.3,120.4 \mathrm{IR} v 3083(\mathrm{w})$, 3060 (w), 2867 (w), 2402 (w), 1601 (m), 1585 (m), 1564 (m), 1440 (m), 1338 (s), 1302 (m), 1148 (m), 1018 (w), $834(\mathrm{~m}), 798(\mathrm{w}), 740(\mathrm{~s}), 694(\mathrm{~s}), 674(\mathrm{~m}), 649(\mathrm{~m})$. The characterization data for compounds 4 corresponded to the reported values. ${ }^{4}$

\section{1-[(Triisopropyllsilyl)ethynyl]-1,2-benziodoxol-3(1H)-one (TIPS-EBX, 1)}

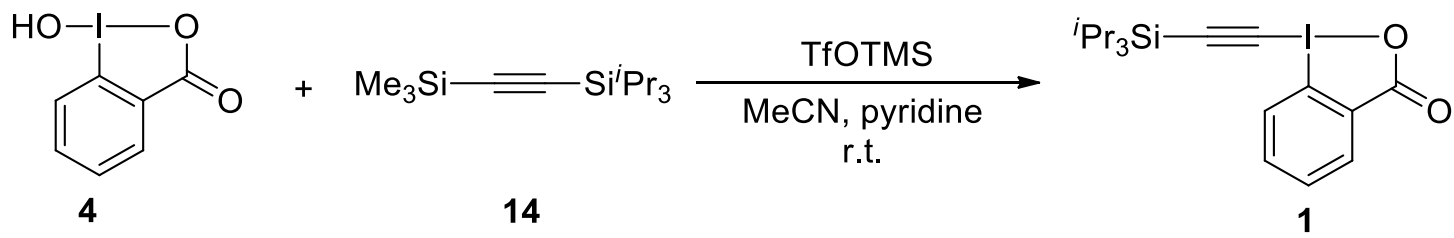

2-iodosylbenzoic acid (4) (21.7 g, $82.0 \mathrm{mmol}, 1.0$ equiv) was charged in oven-dried three-neck 1L flask equipped with a magnetic stirred. After 3 vacuum/nitrogen cycles, anhydrous acetonitrile $(500 \mathrm{~mL})$ was

\footnotetext{
${ }^{3}$ Helal, C J.; Magriotis, P. A; Corey, E. J. J. Am. Chem. Soc. 1996, 118, 10938.

${ }^{4}$ Kraszkiewicz, L.; Skulski, L. Arkivoc 2003, 6, 120.
} 
added via canula and cooled to $4{ }^{\circ} \mathrm{C}$. Trimethylsilyltriflate $(16.4 \mathrm{~mL}, 90.0 \mathrm{mmol}, 1.1$ equiv) was added dropwise via a dropping funnel over $30 \mathrm{~min}$ (no temperature increase was observed). After $15 \mathrm{~min}$, (trimethylsilyl)(triisopropylsilyl)acetylene (14) (23.0 g, $90.0 \mathrm{mmol}, 1.1$ equiv) was added via canula over 15 min (no temperature increase was observed). After $30 \mathrm{~min}$, the suspension became an orange solution. After $10 \mathrm{~min}$, pyridine $(7.0 \mathrm{~mL}, 90 \mathrm{mmol}, 1.1$ equiv) was added via syringe. After $15 \mathrm{~min}$, the reaction mixture was transferred in a one-neck $1 \mathrm{~L}$ flask and reduced under vacuum until a solid was obtained. The solid was dissolved in $\mathrm{CH}_{2} \mathrm{Cl}_{2}(200 \mathrm{~mL})$ and transferred in a $1 \mathrm{~L}$ separatory funnel. The organic layer was added and washed with $1 \mathrm{M} \mathrm{HCl}(200 \mathrm{~mL})$ and the aqueous layer was extracted with $\mathrm{CH}_{2} \mathrm{Cl}_{2}(200 \mathrm{~mL})$. The organic layers were combined, washed with a saturated solution of $\mathrm{NaHCO}_{3}(2$ x $200 \mathrm{~mL})$, dried over $\mathrm{MgSO}_{4}$, filtered and the solvent was evaporated under reduced pressure. Recrystallization from acetonitrile ( $\mathrm{ca} 120$ $\mathrm{mL})$ afforded $1(30.1 \mathrm{~g}, 70.2 \mathrm{mmol}, 86 \%)$ as colorless cristals. Mp (Dec.) 170-176 ${ }^{\circ} \mathrm{C}{ }^{1} \mathrm{H} \mathrm{NMR}(400 \mathrm{MHz}$, $\left.\mathrm{CDCl}_{3}\right) \delta 8.44(\mathrm{~m}, 1 \mathrm{H}, \mathrm{ArH}), 8.29(\mathrm{~m}, 1 \mathrm{H}, \mathrm{ArH}), 7.77$ (m, $\left.2 \mathrm{H}, \mathrm{ArH}\right), 1.16$ (m, $\left.21 \mathrm{H}, \mathrm{TIPS}\right) .{ }^{13} \mathrm{C}$ NMR $(100$ $\left.\mathrm{MHz}, \mathrm{CDCl}_{3}\right) \delta 166.4,134.6,132.3,131.4,131.4,126.1,115.6,114.1,64.6,18.4,11.1$. IR v $2943(\mathrm{~m}), 2865$ (m), 1716 (m), 1618 (m), 1604 (s), 1584 (m), 1557 (m), 1465 (m), 1439 (w), 1349 (m), 1291 (m), 1270 (w), $1244(\mathrm{~m}), 1140(\mathrm{~m}), 1016(\mathrm{~m}), 999(\mathrm{~m}), 883(\mathrm{~m}), 833(\mathrm{~m}), 742(\mathrm{~m}), 702(\mathrm{~s}), 636(\mathrm{~m})$. Characterization data of $\mathbf{1}$ corresponded to the literature values. ${ }^{5}$ The crystal structure of $\mathbf{5 d}$ has been deposited at the Cambridge Crystallographic Data Centre and allocated the deposition number: CCDC 863342.

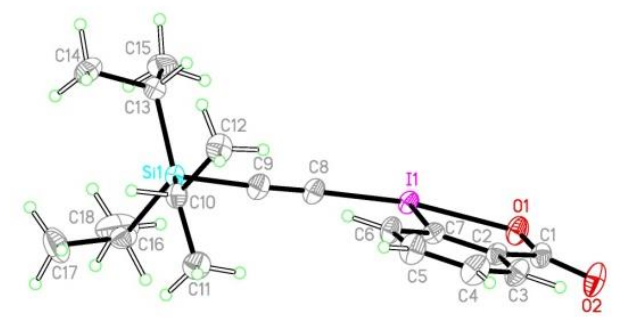

1-[(Trimethylsilyl)ethynyl]-1,2-benziodoxol-3(1H)-one (TMS-EBX, 5a)

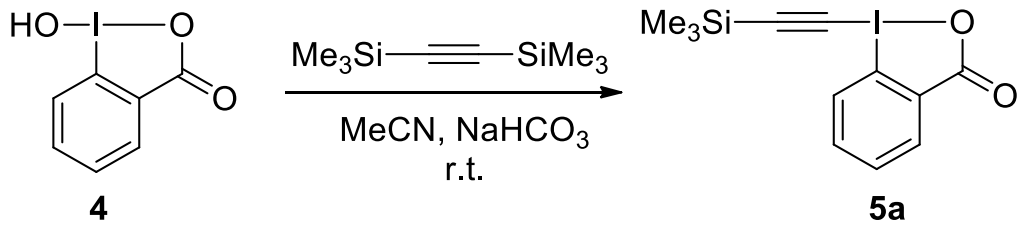

Following a slight modification of the reported procedure, ${ }^{5}$ trimethylsilyl triflate $(5.54 \mathrm{~mL}, 30.7 \mathrm{mmol}, 1.1$ equiv) was added to a suspension of 2-iodosylbenzoic acid (4) (7.36 g, $28.0 \mathrm{mmol}, 1$ equiv) in $\mathrm{CH}_{2} \mathrm{Cl}_{2}(85$ $\mathrm{mL})$ at RT. The resulting yellow mixture was stirred for $1 \mathrm{~h}$, followed by the dropwise addition of bis(trimethylsilyl)acetylene $(6.98 \mathrm{~mL}, 30.7 \mathrm{mmol}, 1.1$ equiv). The resulting suspension was stirred for $6 \mathrm{~h}$ at RT, during this time a white solid was formed. A saturated solution of $\mathrm{NaHCO}_{3}$ was then added and the mixture was stirred vigorously until completely solubilization of the white solid. The two layers were separated and the combined organic extracts were washed with sat. $\mathrm{NaHCO}_{3}$, dried over $\mathrm{MgSO}_{4}$, filtered and evaporated under reduced pressure. Recrystallization from acetonitrile $(5 \mathrm{~mL})$ afforded $\mathbf{5 a}(7.17 \mathrm{~g}, 20.8$ mmol, $74 \%)$ as a colorless solid. ${ }^{1} \mathrm{H}$ NMR $\left(400 \mathrm{MHz}, \mathrm{CDCl}_{3}\right) \delta 8.42(\mathrm{dd}, J=6.4,1.9 \mathrm{~Hz}, 1 \mathrm{H}, \operatorname{Ar} H), 8.19$ $(\mathrm{m}, 1 \mathrm{H}, \operatorname{Ar} H), 7.78$ (m, $2 \mathrm{H}, \operatorname{Ar} H), 0.32$ (s, $9 \mathrm{H}, \mathrm{TMS}) .{ }^{13} \mathrm{C} \mathrm{NMR}\left(100 \mathrm{MHz}, \mathrm{CDCl}_{3}\right)$ 166.4, 134.9, 132.6, 131.7, 131.4, 126.1, 117.2, 115.4, 64.2, -0.5. IR v 3389 (w), 2967 (w), 1617 (s), 1609 (s), 1562 (m), 1440 (w), $1350(\mathrm{~m}), 1304$ (w), 1254 (w), 1246 (w), $1112(\mathrm{w}), 1008$ (w), 852 (s), $746(\mathrm{~m}), 698$ (m), 639 (m). The

${ }^{5}$ Zhdankin, V. V.; Kuehl, C. J.; Krasutsky, A. P.; Bolz, J. T.; Simonsen, A. J. J. Org. Chem. 1996, 61, 6547. 
characterization data for compounds 14 corresponded to the reported values. ${ }^{5} \mathrm{~A} \mathrm{X}$-ray structure is available as a separate cif file. CCDC 863343

\section{Triethyl trimethylsilylacetylene (33)}

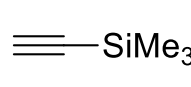

32

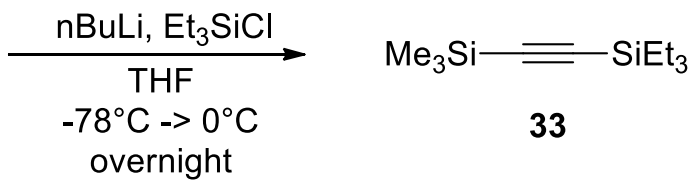

Following a reported procedure, ${ }^{3} n$-butyllithium $(2.5 \mathrm{M}$ in hexanes, $5.4 \mathrm{~mL}, 14 \mathrm{mmol}, 1.0$ equiv) was added dropwise to a stirred solution of ethynyltrimethylsilane (32) (1.36 g, $13.8 \mathrm{mmol}, 1.00$ equiv) in THF $(21 \mathrm{~mL})$ at $-78{ }^{\circ} \mathrm{C}$. The mixture was then warmed to $0{ }^{\circ} \mathrm{C}$ and stirred for $5 \mathrm{~min}$. The mixture was then cooled back to $-78{ }^{\circ} \mathrm{C}$ and chlorotriethylsilane $(2.3 \mathrm{~mL}, 14 \mathrm{mmol}, 0.98$ equiv) was added dropwise. The mixture was then allowed to warm to room temperature and stirred overnight. A saturated solution of ammonium chloride $(20 \mathrm{~mL})$ was added, and the reaction mixture was extracted with diethyl ether $(2 \mathrm{x} 20 \mathrm{~mL})$. The organic layer was washed with water and brine, then dried over $\mathrm{MgSO}_{4}$, filtered and concentrated under reduced pressure to obtain a colorless liquid which was further purified by Kugelrohr distillation to yield 33 (3.4 g, $11 \mathrm{mmol}, 83 \%$ yield) as a colorless liquid. ${ }^{1} \mathrm{H} \mathrm{NMR}\left(400 \mathrm{MHz}, \mathrm{CDCl}_{3}\right) 0.99$ (t, J=7.9 Hz, $9 \mathrm{H}$, $\mathrm{SiCH}_{2} \mathrm{CH}_{3}$ ), 0.59 (q, $J=7.9 \mathrm{~Hz}, 6 \mathrm{H}, \mathrm{SiCH}_{2} \mathrm{CH}_{3}$ ), 0.17 (s, $\left.9 \mathrm{H}, \mathrm{TMS}\right) .{ }^{13} \mathrm{C} \mathrm{NMR}\left(100 \mathrm{MHz}, \mathrm{CDCl}_{3}\right) \square \delta \square$ 115.4, 111.2, 7.4, 4.4, 0.0. IR v 2958 (m), 2913 (m), 2879 (m), 1462 (w), 1414 (w), $1381(\mathrm{w}), 1250$ (m), 1015 (m), 973 (w), 908 (w), 844 (s), 773 (s), 731 (s), 702 (sh), 679 (sh). Consistent with reported data. ${ }^{6}$

\section{1-[(Triethylsilyl)ethynyl]-1,2-benziodoxol-3(1H)-one (5b)}<smiles>O=C1OI(O)c2ccccc21</smiles>

4

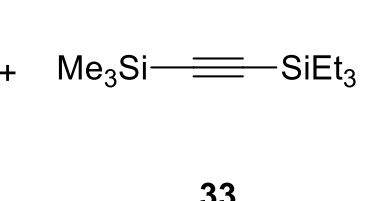

33

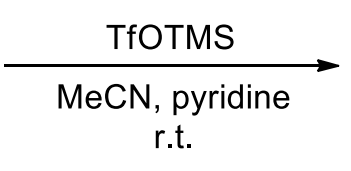

r.t.

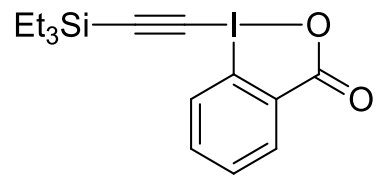

$5 b$

Trimethylsilyltriflate $\left(2.78 \mathrm{~mL}, 15.4 \mathrm{mmol}, 1.1\right.$ equiv, freshly distilled over $\left.\mathrm{CaH}_{2}\right)$ was added dropwise to a stirred solution of 2-iodosylbenzoic acid (4) (3.71 g, $14.0 \mathrm{mmol}, 1.0$ equiv) in acetonitrile $(50 \mathrm{~mL})$. After $15 \mathrm{~min}$, (trimethylsilyl)(triethylsilyl)acetylene (33) (3.26 g, $15.4 \mathrm{mmol}, 1.1$ equiv) was then added dropwise. After $30 \mathrm{~min}$ pyridine $(1.25 \mathrm{~mL}, 15.4 \mathrm{mmol}, 1.1$ equiv) was added and the mixture was stirred for an additional $15 \mathrm{~min}$. The solvent was then removed under reduced pressure and the yellow crude oil was dissolved in dichloromethane $(50 \mathrm{~mL})$. The organic layer was washed with $1 \mathrm{M} \mathrm{HCl}(50 \mathrm{~mL})$, and the aqueous layer was extracted with $\mathrm{CH}_{2} \mathrm{Cl}_{2}(50 \mathrm{~mL})$. The organic layers were washed twice with saturated $\mathrm{NaHCO}_{3}(75 \mathrm{~mL})$, dried over $\mathrm{MgSO}_{4}$, filtered and the solvent was evaporated under reduced pressure. The resulting solid was recristalized twice in $\mathrm{CH}_{3} \mathrm{CN}$. The solid was washed with cold acetonitirile, hexanes and dried under high vacuum to afford $\mathbf{5 b}$ (2.95 g, $7.64 \mathrm{mmol}, 55 \%$ yield) as a slightly brown solid. Mp (Dec.) $155-158^{\circ} \mathrm{C} .{ }^{1} \mathrm{H}$ NMR $\left(400 \mathrm{MHz}, \mathrm{CDCl}_{3}\right) \delta 8.40(\mathrm{~m}, 1 \mathrm{H}, \mathrm{Ar} H), 8.24(\mathrm{~m}, 1 \mathrm{H}, \mathrm{ArH}), 7.75(\mathrm{~m}, 2 \mathrm{H}, \mathrm{Ar} H)$, $1.06\left(\mathrm{t}, J=8.0 \mathrm{~Hz}, 9 \mathrm{H}, \mathrm{SiCH}_{2} \mathrm{CH}_{3}\right), 0.73\left(\mathrm{q}, J=8.0 \mathrm{~Hz} ; 6 \mathrm{H}, \mathrm{SiCH}_{2} \mathrm{CH}_{3}\right) .{ }^{13} \mathrm{C} \mathrm{NMR}\left(100 \mathrm{MHz}, \mathrm{CDCl}_{3}\right) \delta$ 166.5, 134.8, 132.5, 131.6, 131.3, 126.1, 115.5, 115.1, 64.6, 7.4, 4.1. IR v $3064(\mathrm{w}), 3062(\mathrm{~m}), 2957(\mathrm{~m})$, $2911(\mathrm{~m}), 2877(\mathrm{~m}), 1621(\mathrm{~s}), 1587(\mathrm{~m}), 1561(\mathrm{~m}), 1460(\mathrm{~m}), 1440(\mathrm{~m}), 1415(\mathrm{w}), 1378(\mathrm{w}), 1336(\mathrm{~m}), 1297$ (m), 1237 (w), 1149 (w), 1113 (w), 1010 (m), 976 (w), 912 (w), 912 (w), 834 (m), 804 (w), 739 (s), 693 (m), $675(\mathrm{~m}), 647(\mathrm{w})$. HRMS (ESI) calcd for $\mathrm{C}_{15} \mathrm{H}_{20} \mathrm{IO}_{2} \mathrm{Si}^{+}(\mathrm{M}+\mathrm{H})$ 387.0277; found 387.0290. Consistent with reported data. ${ }^{6}$ The crystal structure of $\mathbf{5 d}$ has been deposited at the Cambridge Crystallographic Data Centre and allocated the deposition number: CCDC 863344.

\footnotetext{
${ }^{6}$ Nicolai, S.; Piemontesi, C.; Waser, J. Angew. Chem., Int. Ed. 2011, 50, 4680
} 


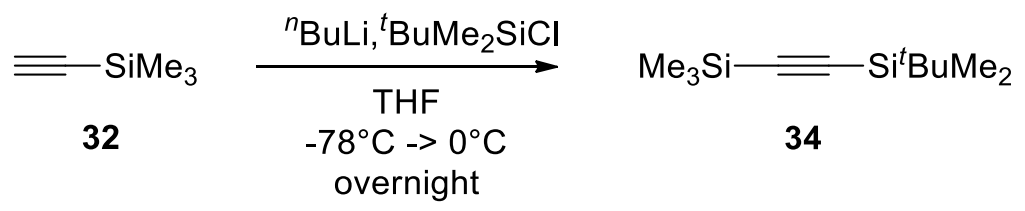

Following a reported procedure, $3 n$-butyllithium $(2.5 \mathrm{~m}$ in hexanes, $4.0 \mathrm{~mL}, 10 \mathrm{mmol}, 1.0$ equiv) was added dropwise to a stirred solution of ethynyltrimethylsilane (32) $(1.42 \mathrm{~mL}, 10.0 \mathrm{mmol}, 1.0$ equiv) in THF $(10 \mathrm{~mL})$ at $-78{ }^{\circ} \mathrm{C}$. After $15 \mathrm{~min}$, tert-butyldimethylsilyl chloride $(1.51 \mathrm{~g}, 10.0 \mathrm{mmol}, 1.0$ equiv $)$ dissolved in THF ( $2 \mathrm{~mL})$ was added dropwise. The mixture was then allowed to warm to room temperature and stirred for $3 \mathrm{~h}$. A saturated solution of ammonium chloride $(30 \mathrm{~mL})$ was added, and the reaction mixture was extracted with diethyl ether $(2 \times 30 \mathrm{~mL})$, then dried over $\mathrm{MgSO}_{4}$, filtered and concentrated under reduced pressure to obtain $34\left(2.02 \mathrm{~g}, 9.50 \mathrm{mmol}, 95 \%\right.$ yield) as a colorless liquid. ${ }^{1} \mathrm{H}$ NMR $\left(400 \mathrm{MHz}, \mathrm{CDCl}_{3}\right) \delta$ 0.93 (s, 9H, $t \mathrm{Bu}), 0.17$ (s, $\left.9 \mathrm{H}, \mathrm{SiMe}_{3}\right), 0.10$ (s, $6 \mathrm{H}, \mathrm{SiMe}_{2}$ ).

\section{1-[(tertButyldimethylsilyl)ethynyl]-1,2-benziodoxol-3(1H)-one (5c)}<smiles>O=C1OI(O)c2ccccc21</smiles>

4

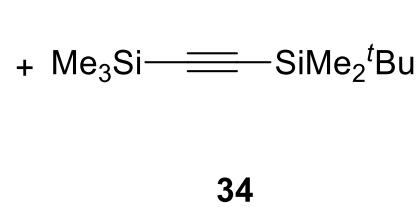

34<smiles>CC(C)(C)[SiH2]C#CI1OC(=O)c2ccccc21</smiles>

5c

Trimethylsilyltriflate $\left(775 \mu \mathrm{L}, 4.15 \mathrm{mmol}, 1.1\right.$ equiv, freshly distilled over $\left.\mathrm{CaH}_{2}\right)$ was added dropwise to a stirred solution of 2-iodosylbenzoic acid (4) (1.00 g, $3.77 \mathrm{mmol}, 1.0$ equiv) in acetonitrile (20 mL). After $15 \mathrm{~min}, 34$ ( $881 \mathrm{mg}, 4.15 \mathrm{mmol}, 1.1$ equiv) was then added dropwise. After 20 min pyridine ( $340 \mu \mathrm{L}, 4.15$ mmol, 1.1 equiv) was added and the mixture was stirred for an additional $15 \mathrm{~min}$. The solvent was then removed under reduced pressure and the yellow crude oil was dissolved in dichloromethane $(20 \mathrm{~mL})$. The organic layer was washed with $1 \mathrm{M} \mathrm{HCl}(20 \mathrm{~mL})$, and the aqueous layer was extracted with $\mathrm{CH}_{2} \mathrm{Cl}_{2}(20 \mathrm{~mL})$. The organic layers were washed with saturated $\mathrm{NaHCO}_{3}(30 \mathrm{~mL})$, dried over $\mathrm{MgSO}_{4}$, filtered and the solvent was evaporated under reduced pressure. The resulting solid was recrystalized twice in $\mathrm{CH}_{3} \mathrm{CN}$ (ca $10 \mathrm{~mL}$ ), washed with hexanes and dried under high vacuum to afford $\mathbf{5 c}$ (795 $\mathrm{mg}, 2.06 \mathrm{mmol}$, 55\% yield) as a slightly brown solid. Mp (Dec.) $164-166^{\circ} \mathrm{C} .{ }^{1} \mathrm{H} \mathrm{NMR}\left(400 \mathrm{MHz}, \mathrm{CDCl}_{3}\right)(\mathrm{ca} 0.06 \mathrm{mmol} / \mathrm{ml}) \delta 8.40(\mathrm{~m}, 1$ $\mathrm{H}, \mathrm{ArH}), 8.21(\mathrm{~m}, 1 \mathrm{H}, \mathrm{ArH}), 7.76(\mathrm{~m}, 2 \mathrm{H}, \mathrm{ArH}), 1.01(\mathrm{~s}, 9 \mathrm{H}, t \mathrm{Bu}), 0.24(\mathrm{~s}, 6 \mathrm{H}, \mathrm{Me}) .{ }^{13} \mathrm{C} \mathrm{NMR}(101 \mathrm{MHz}$, $\left.\mathrm{CDCl}_{3}\right) \delta 166.3,134.8,132.5,131.6,131.4,126.0,115.8,115.5,64.5,26.0,16.7,-4.8 . \mathrm{IR} 2953(\mathrm{w}), 2930$ (w), $2858(w), 2244(\mathrm{w}), 1652$ (s), 1626 (s), 1608 (s), 1588 (w), 1560 (w), 1469 (w), 1462 (w), 1442 (w), 1335 (m), 1295 (w), 1250 (w), 1167 (w), 1009 (w), 910 (m), 843 (s), 824 (s), 813 (s), 783 (m), 741 (s), 734 (s), $703(\mathrm{~s}), 667$ (m). ). HRMS(ESI) calcd for $\mathrm{C}_{15} \mathrm{H}_{20} \mathrm{O}_{2} \mathrm{ISi}^{+}(\mathrm{M}+\mathrm{H}) 387.0277$, found 387.0283. The crystal structure of 5d has been deposited at the Cambridge Crystallographic Data Centre and allocated the deposition number: CCDC 863345.

tertButyldiphenylsilyl trimethylsilylacetylene (35)

\begin{tabular}{|c|c|c|}
\hline SiMe & nBuLi, ${ }^{t} \mathrm{BuPh}_{2} \mathrm{SiCl}$ & \\
\hline Silvie & THF & 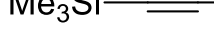 \\
\hline 32 & $\begin{array}{c}-78^{\circ} \mathrm{C}->0{ }^{\circ} \mathrm{C} \\
\text { overnight }\end{array}$ & 35 \\
\hline
\end{tabular}


Following a reported procedure, ${ }^{7} n$-butyllithium $(2.5 \mathrm{M}$ in hexanes, $8.0 \mathrm{~mL}, 20.0 \mathrm{mmol}, 0.98$ equiv) was added dropwise to a stirred solution of ethynyltrimethylsilane (32) $(2.90 \mathrm{~mL}, 20.4 \mathrm{mmol}, 1.0$ equiv) in THF $\left(30 \mathrm{~mL}\right.$ ) at $-78{ }^{\circ} \mathrm{C}$. The mixture was then warmed to $0{ }^{\circ} \mathrm{C}$ and stirred for $5 \mathrm{~min}$. The mixture was then cooled back to $-78{ }^{\circ} \mathrm{C}$ and tert-butylchlorodiphenylsilane $(6.4 \mathrm{~mL}, 30 \mathrm{mmol}, 1.0$ equiv) was added dropwise. The mixture was then allowed to warm to room temperature and stirred overnight. A saturated solution of ammonium chloride $(30 \mathrm{~mL})$ was added, and the reaction mixture was extracted with diethyl ether $(2 \times 50 \mathrm{~mL})$. The organic layer was washed with water and brine, then dried over $\mathrm{MgSO}_{4}$, filtered and concentrated under reduced pressure to obtain a colorless liquid which was further purified by Kugelrohr distillation $\left(\mathrm{bp}=150^{\circ} \mathrm{C}, \mathrm{p}=0.25 \mathrm{mmHg}\right)$ to yield $35(2.95 \mathrm{~g}, 8.76 \mathrm{mmol}, 44 \%$ yield $)$ as a colorless liquid. ${ }^{1} \mathrm{H}$ NMR (400 MHz, $\left.\mathrm{CDCl}_{3}\right) \delta 7.80$ (dd, $\left.4 \mathrm{H}, \mathrm{m}, \mathrm{ArH}\right), 7.38(\mathrm{~m}, 6 \mathrm{H}, \mathrm{ArH}), 1.08$ (s, $\left.9 \mathrm{H}, \mathrm{tBu}\right), 0.27$ (s, $9 \mathrm{H}$, TMS). ${ }^{13} \mathrm{C}$ NMR $\left(101 \mathrm{MHz}, \mathrm{CDCl}_{3}\right) \delta 135.6,133.2,129.5,127.7,119.0,108.7,27.0,18.5$, -0.0. The characterization data for compound $\mathbf{x}$ corresponded to the reported values. ${ }^{7}$

1-[(tertButyldiphenylsilyl)ethynyl]-1,2-benziodoxol-3(1H)-one (5d)<smiles>O=C1OI(O)c2ccccc21</smiles>

4

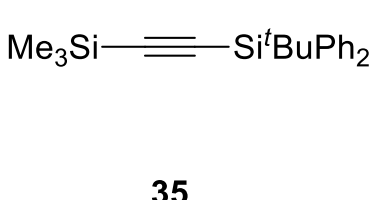

35

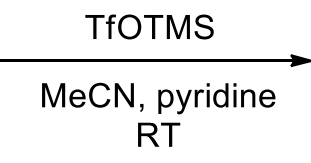
RT

Trimethylsilyltriflate $(1.58 \mathrm{~mL}, 8.70 \mathrm{mmol}, 1.1$ equiv, freshly distilled) was added dropwise to a stirred solution of 2-iodosylbenzoic acid (4) $(2.07 \mathrm{~g}, 7.90 \mathrm{mmol}, 1.0$ equiv) in acetonitrile (30 mL). tertbutyldiphenyl((trimethylsilyl)ethynyl)silane (35) (2.95 g, $3.70 \mathrm{mmol}, 1.1$ equiv) was then added dropwise, followed, after $15 \mathrm{~min}$, by the addition of pyridine $(713 \mu \mathrm{L}, 3.70 \mathrm{mmol}, 1.1$ equiv). The mixture was stirred $10 \mathrm{~min}$. The solvent was then removed under reduced pressure and the yellow crude oil was dissolved in dichloromethane. The organic layer was washed with $1 \mathrm{M} \mathrm{HCl}$ and the aqueous layer was extracted with $\mathrm{CH}_{2} \mathrm{Cl}_{2}$. The organic layers were combined, washed with a saturated solution of $\mathrm{NaHCO}_{3}$, dried over $\mathrm{MgSO}_{4}$, filtered and the solvent was evaporated under reduced pressure. The resulting oil was stirred in hexane and ether and then reduced under vacuum to afford a colorless solid. Recrystallization from acetonitrile ( $\mathrm{ca} 20 \mathrm{~mL}$ ) afforded $\mathbf{5 d}(2.77 \mathrm{~g}, 5.42 \mathrm{mmol}, 69 \%)$ as a colorless solid. Mp (Dec.) $157-159^{\circ} \mathrm{C}$. ${ }^{1} \mathrm{H} \mathrm{NMR}\left(400 \mathrm{MHz}, \mathrm{CDCl}_{3}\right)(\mathrm{ca} 0.12 \mathrm{mmol} / \mathrm{ml}) \delta 8.43(\mathrm{~d}, 1 \mathrm{H}, J=6.5 \mathrm{~Hz}, \mathrm{ArH}), 8.29(\mathrm{~d}, 1 \mathrm{H}, J=8.2 \mathrm{~Hz}$, ArH), $7.82(\mathrm{~d}, 4 \mathrm{H}, J=6.6 \mathrm{~Hz}, \mathrm{ArH}), 7.75(\mathrm{t}, 1 \mathrm{H}, J=7.2 \mathrm{~Hz}, \mathrm{ArH}), 7.66$ (m, $1 \mathrm{H}, \mathrm{ArH}), 7.53-7.41(\mathrm{~m}, 6 \mathrm{H}$, $\mathrm{ArH}), 1.21(\mathrm{~s}, 9 \mathrm{H}, t \mathrm{Bu}) .{ }^{13} \mathrm{C} \mathrm{NMR}\left(101 \mathrm{MHz}, \mathrm{CDCl}_{3}\right) \delta 166.6,135.5,134.8,132.4,131.5,131.3,130.2$, 128.1, 126.3, 116.0, 112.2, 68.5, 27.0, 18.7. One carbon was not resolved. IR 3072 (w), $2958(w), 2932$ (w), 2865 (w), 2860 (w), 2248 (w), 1649 (w), 1622 (m), $1561(w), 1471(w), 1430(w), 1336(w), 1297(w), 1253$ (w), 1113 (w), 1008 (w), 906 (s), 821 (w), 727 (s), 647 (m). HRMS(ESI) calcd for $\mathrm{C}_{25} \mathrm{H}_{24} \mathrm{O}_{2} \mathrm{ISi}^{+}(\mathrm{M}+\mathrm{H})$ 511.0590, found 511.0569. The crystal structure of $\mathbf{5 d}$ has been deposited at the Cambridge Crystallographic Data Centre and allocated the deposition number: CCDC 863346

\section{Reaction with silyl alkynyl benziodoxolones}

\section{3-((Trimethylsilyl)ethynyl)-1H-indole (7a)}

\footnotetext{
${ }^{7}$ Cuadrado, P.; Gonzalez-Nogal, A. M.; Valero, R. Tetrahedron 2002, 58, 4975.
} 


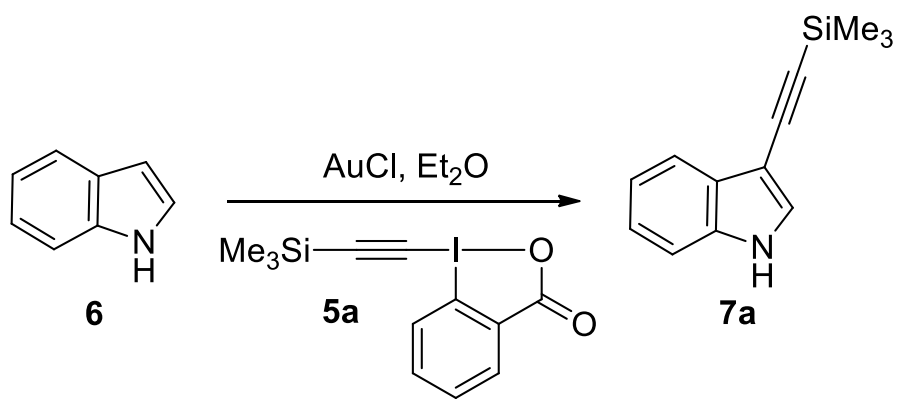

Problems of reproducibility of this reaction was observed yield from 0 to $23 \%$ ). The best result obtained is reported here.

TMS-EBX (5a) ( $83 \mathrm{mg}, 0.24 \mathrm{mmol}, 1.2$ equiv) was added to a stirring solution of $\mathrm{AuCl}$ (2.3 mg, 0.010 mmol, 0.05 equiv) and indole (6) (23.4 mg, 0.200 mmol, 1.0 equiv) in $\mathrm{Et}_{2} \mathrm{O} 1$ (4 mL) under air. The reaction was sealed and stirred at room temperature for $16 \mathrm{~h}$. $\mathrm{Et}_{2} \mathrm{O}(10 \mathrm{~mL})$ was added, the organic layer was washed twice with $0.1 \mathrm{M} \mathrm{NaOH}(15 \mathrm{~mL})$. The aqueous layers were combined and extracted with $\mathrm{Et}_{2} \mathrm{O}(20 \mathrm{~mL})$. The organic layers were combined, washed with saturated $\mathrm{NaHCO}_{3}(20 \mathrm{~mL})$, brine $(20 \mathrm{~mL})$, dried with $\mathrm{MgSO}_{4}$ and concentrated under reduced pressure. Purification by flash chromatography (PET/EtOAc 95/5) afforded 7a (10 mg, $0.047 \mathrm{mmol}, 23 \%)$ as amorphous colorless solid. $\mathrm{R}_{f} 0.15$ (PET/EtOAc 95/5, UV). ${ }^{1} \mathrm{H}$ NMR (400 $\left.\mathrm{MHz} \mathrm{CDCl}_{3}\right) \delta 8.16$ (br s, $\left.1 \mathrm{H}, \mathrm{NH}\right), 7.75(\mathrm{dm}, J=7.0 \mathrm{~Hz}, 1 \mathrm{H}, \mathrm{ArH}), 7.43(\mathrm{~m}, 1 \mathrm{H}, \mathrm{ArH}), 7.37(\mathrm{dm}, J=8.2$ $\mathrm{Hz}, 1 \mathrm{H}, \mathrm{ArH}), 7.22$ (m, $2 \mathrm{H}, \mathrm{ArH}), 0.29$ (s, $9 \mathrm{H}, \mathrm{TMS}) .{ }^{13} \mathrm{C} \mathrm{NMR}\left(101 \mathrm{MHz}, \mathrm{CDCl}_{3}\right) \delta$ 135.0, 128.4, 128.2, 123.1, 120.7, 120.1, 111.2, 98.9, 98.5, 95.8, 0.3. IR 3412 (m), $3119(\mathrm{w}), 3060(\mathrm{w}), 2954(\mathrm{w}), 2898(\mathrm{w}), 2516$ (w), $2155(\mathrm{~m}), 1718(\mathrm{w}), 1629(\mathrm{w}), 1529(\mathrm{w}), 1489(\mathrm{w}), 1457$ (m), $1417(\mathrm{w}), 1325(\mathrm{w}), 1238(\mathrm{~s}), 1128(\mathrm{w})$, $1103(\mathrm{w}), 1070(\mathrm{~m}), 1007(\mathrm{w}), 839(\mathrm{~s}), 817(\mathrm{~m}), 760(\mathrm{~m}), 741(\mathrm{~s}), 703(\mathrm{~m})$. HRMS (ESI) calcd for $\mathrm{C}_{13} \mathrm{H}_{16} \mathrm{NSi}^{+}[\mathrm{M}+\mathrm{H}]^{+}$214.1047; found 214.1051.

\section{3-((Triethylsilyl)ethynyl)-1H-indole (7b)}
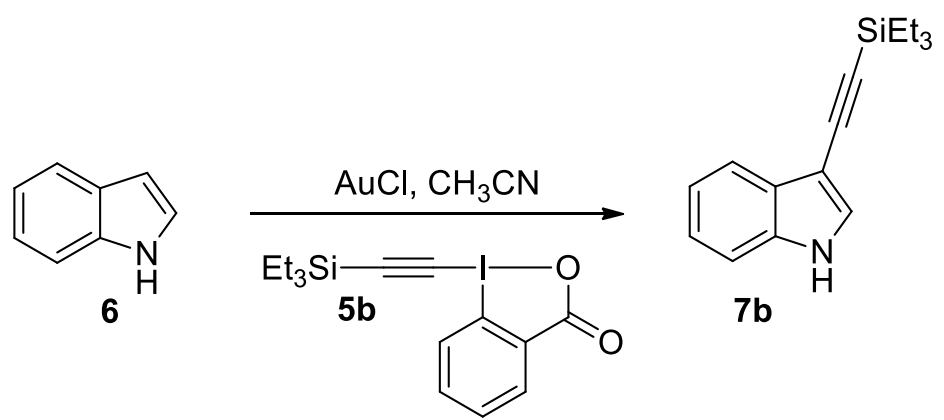

TES-EBX (5b) (186 mg, $0.240 \mathrm{mmol}, 1.2$ equiv) was added to a stirring solution of $\mathrm{AuCl}$ (4.6 mg, 0.020 mmol, 0.05 equiv) and indole (6) $\left(47 \mathrm{mg}, 0.40 \mathrm{mmol}, 1.0\right.$ equiv) in $\mathrm{CH}_{3} \mathrm{CN} 1(8 \mathrm{~mL})$ under air. The reaction was sealed and stirred at room temperature for $15 \mathrm{~h}$. $\mathrm{Et}_{2} \mathrm{O}(10 \mathrm{~mL})$ was added, the organic layer was washed twice with $0.1 \mathrm{M} \mathrm{NaOH}(15 \mathrm{~mL})$. The aqueous layers were combined and extracted with $\mathrm{Et}_{2} \mathrm{O}(20 \mathrm{~mL})$. The organic layers were combined, washed with saturated $\mathrm{NaHCO}_{3}(20 \mathrm{~mL})$, brine $(20 \mathrm{~mL})$, dried with $\mathrm{MgSO}_{4}$ and concentrated under reduced pressure. Purification by column chromatography (pentane/Et ${ }_{2} \mathrm{O} 8 / 2$ ) afforded $7 \mathbf{b}\left(58 \mathrm{mg}, 0.23 \mathrm{mmol}, 57 \%\right.$ ) as a red oil. $\mathrm{R}_{\mathrm{f}}$ (pentane/Et $\left.\mathrm{E}_{2} \mathrm{O} 95 / 5\right): 0.15 .{ }^{1} \mathrm{H} \mathrm{NMR}(400 \mathrm{MHz}$, $\left.\mathrm{CDCl}_{3}\right) \delta 8.14$ (br s, $\left.1 \mathrm{H}, \mathrm{NH}\right), 7.80(\mathrm{~m}, 1 \mathrm{H}, \mathrm{ArH}), 7.40$ (d, $\left.1 \mathrm{H}, J=2.6 \mathrm{~Hz}, \mathrm{ArH}\right), 7.36(\mathrm{~m}, 1 \mathrm{H}, \mathrm{ArH}), 7.27$

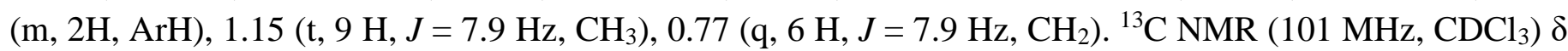
134.9, 128.7, 128.3, 123.0, 120.7, 119.9, 111.3, 99.7, 98.9, 93.1, 7.6, 4.6. IR 3406 (m), 2955 (m), $2910(\mathrm{w})$, $2875(\mathrm{~m}), 2151$ (m), 1620 (w), 1538 (w), 1457 (m), 1414 (m), 1341 (w), 1325 (w), 1239 (m), 1129 (w), 
1072 (w), 1008 (m), 976 (w), 909 (m), 816 (w), 778 (s), 734 (s), 695 (m). HRMS (ESI) calcd for $\mathrm{C}_{16} \mathrm{H}_{22} \mathrm{NSi}^{+}$ $[\mathrm{M}+\mathrm{H}]^{+} 256.1516$; found 256.1512 .

\section{3-((tert-Butyldimethylsilyl)ethynyl)-1H-indole (7c)}

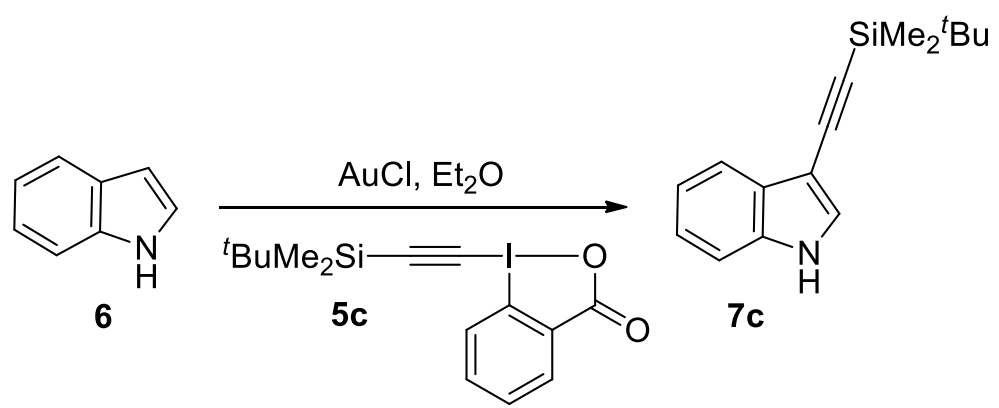

5c (93 mg, $0.24 \mathrm{mmol}, 1.2$ equiv) was added to a stirring solution of $\mathrm{AuCl}$ ( $2.3 \mathrm{mg}, 0.010 \mathrm{mmol}, 0.05$ equiv) and indole (6) (23.4 mg, $0.200 \mathrm{mmol}, 1.0$ equiv) in $\mathrm{Et}_{2} \mathrm{O} 1(4 \mathrm{~mL})$ under air. The reaction was sealed and stirred at room temperature for $16 \mathrm{~h}$. $\mathrm{Et}_{2} \mathrm{O}(10 \mathrm{~mL})$ was added, the organic layer was washed twice with $0.1 \mathrm{~m} \mathrm{NaOH}(15 \mathrm{~mL})$. The aqueous layers were combined and extracted with $\mathrm{Et}_{2} \mathrm{O}(20 \mathrm{~mL})$. The organic layers were combined, washed with saturated $\mathrm{NaHCO}_{3}(20 \mathrm{~mL})$, brine $(20 \mathrm{~mL})$, dried with $\mathrm{MgSO}_{4}$ and concentrated under reduced pressure. Purification by flash chromatography (PET/EtOAc 95/5) afforded 7c (40 mg, $0.16 \mathrm{mmol}, 78 \%$ ) as amorphous colorless solid. Rf 0.15 (PET/EtOAc 95/5, UV). ${ }^{1} \mathrm{H}$ NMR (400 $\left.\mathrm{MHz}, \mathrm{CDCl}_{3}\right) \delta 8.14$ (br s, $\left.1 \mathrm{H}, J=0.2 \mathrm{~Hz}, \mathrm{NH}\right), 7.76(\mathrm{~m}, 1 \mathrm{H}, \mathrm{ArH}), 7.41(\mathrm{~d}, 1 \mathrm{H}, J=2.6 \mathrm{~Hz}, \mathrm{ArH}), 7.36$ $(\mathrm{m}, 1 \mathrm{H}, \mathrm{ArH}), 7.24(\mathrm{~m}, 2 \mathrm{H}, \mathrm{ArH}), 1.07(\mathrm{~m}, 9 \mathrm{H}, t \mathrm{Bu}), 0.24(\mathrm{~s}, 6 \mathrm{H}, \mathrm{Me}) .{ }^{13} \mathrm{C} \mathrm{NMR}\left(101 \mathrm{MHz}, \mathrm{CDCl}_{3}\right) \delta$ 135.0, 128.7, 128.3, 123.1, 120.7, 120.0, 111.3, 99.2, 99.0, 94.0, 26.2, 16.7, -4.3. IR $3407(\mathrm{w}), 3062(\mathrm{w})$, $2953(\mathrm{~m}), 2929(\mathrm{~m}), 2884(\mathrm{w}), 2860(\mathrm{w}), 2151(\mathrm{~m}), 1723(\mathrm{w}), 1532(\mathrm{w}), 1416(\mathrm{w}), 1361(\mathrm{w}), 1325(\mathrm{w}), 1240$ (m), 1129 (w), 1128 (w), 1072 (w), 1008 (w), 914 (w), 827 (s), 775 (s), 742 (s), 677 (m). HRMS (ESI) calcd for $\mathrm{C}_{16} \mathrm{H}_{22} \mathrm{NSi}^{+}[\mathrm{M}+\mathrm{H}]^{+}$256.1516; found 256.1523.

\section{3-((tert-butyldiphenylsilyl)ethynyl)-1H-indole (7d)}

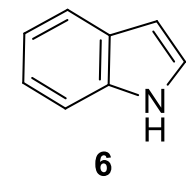

6

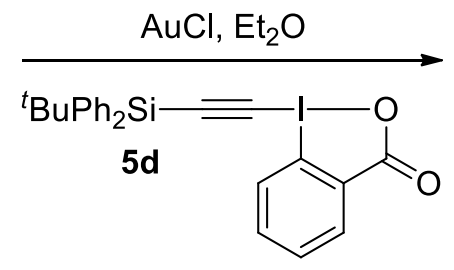

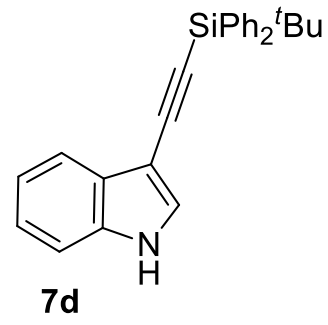

7d

5d (123 mg, $0.240 \mathrm{mmol}, 1.2$ equiv) was added to a stirring solution of $\mathrm{AuCl}$ ( $2.3 \mathrm{mg}, 0.010 \mathrm{mmol}, 0.05$ equiv) and indole (6) (23.4 mg, $0.200 \mathrm{mmol}, 1.0$ equiv) in $\mathrm{Et}_{2} \mathrm{O} 1(4 \mathrm{~mL})$ under air. The reaction was sealed and stirred at room temperature for $16 \mathrm{~h}$. $\mathrm{Et}_{2} \mathrm{O}(10 \mathrm{~mL})$ was added, the organic layer was washed twice with $0.1 \mathrm{M} \mathrm{NaOH}(15 \mathrm{~mL})$. The aqueous layers were combined and extracted with $\mathrm{Et}_{2} \mathrm{O}(20 \mathrm{~mL})$. The organic layers were combined, washed with saturated $\mathrm{NaHCO}_{3}(20 \mathrm{~mL})$, brine $(20 \mathrm{~mL})$, dried with $\mathrm{MgSO}_{4}$ and concentrated under reduced pressure. Purification by flash chromatography (PET/EtOAc 95/5) afforded 7d (69 mg, $0.18 \mathrm{mmol}, 91 \%$ ) as amorphous colorless solid. $\mathrm{R}_{f} 0.15$ (PET/EtOAc 95/5, UV). ${ }^{1} \mathrm{H}$ NMR (400 $\left.\mathrm{MHz} \mathrm{CDCl}_{3}\right) \delta 8.14($ br s, $1 \mathrm{H}, \mathrm{NH}), 7.96(\mathrm{~m}, 4 \mathrm{H}, \mathrm{ArH}), 7.85(\mathrm{~m}, 1 \mathrm{H}, \mathrm{ArH}), 7.50(\mathrm{~d}, 1 \mathrm{H}, J=2.7 \mathrm{~Hz}$, ArH), 7.46-7.34 (m, $7 \mathrm{H}, \mathrm{ArH}), 7.27(\mathrm{~m}, 3 \mathrm{H}, \mathrm{ArH}), 1.22(\mathrm{~m}, 9 \mathrm{H}, \mathrm{tBu}) .{ }^{13} \mathrm{C} \mathrm{NMR}\left(101 \mathrm{MHz}, \mathrm{CDCl}_{3}\right) \delta$ 
135.7, 135.0, 133.9, 129.4, 128.9, 127.7, 123.2, 120.9, 120.1, 111.4, 102.9, 98.9, 90.8, 27.2, 18.7. One C not resolved. IR 3419 (w), 3052 (w), 2959 (w), 2931 (w), 2924 (w), 2857 (w), 2148 (m), 1532 (w), 1459 (w), 1429 (w), 1361 (w), 1326 (w), 1265 (m), 1239 (w), 1190 (w), 1129 (w), 1107 (m), 1073 (w), 1009 (w), 821 (w), 780 (m), 737 (s), 702 (s). HRMS (ESI) calcd for $\mathrm{C}_{26} \mathrm{H}_{26} \mathrm{NSi}^{+}[\mathrm{M}+\mathrm{H}]^{+}$380.1829; found 380.1820 .

\section{3-((Triisopropylsilyl)ethynyl)-1H-indole (7e)}
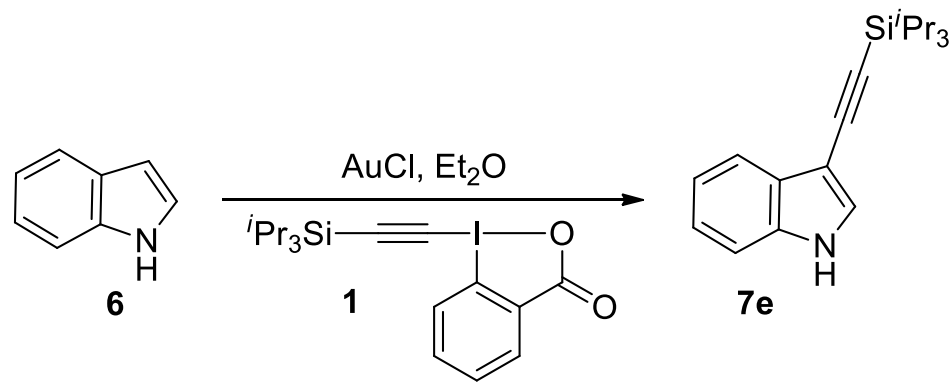

1 (206 mg, $0.480 \mathrm{mmol}, 1.2$ equiv) was added to a stirring solution of $\mathrm{AuCl}$ ( $4.6 \mathrm{mg}, 0.020 \mathrm{mmol}, 0.05$ equiv) and indole (6) (47 mg, $0.400 \mathrm{mmol}, 1.0$ equiv) in $\mathrm{Et}_{2} \mathrm{O}(8 \mathrm{~mL})$ under air. The reaction was sealed and stirred at room temperature for $15 \mathrm{~h}$. $\mathrm{Et}_{2} \mathrm{O}(10 \mathrm{~mL})$ was added, the organic layer was washed twice with $\mathrm{NaOH} 0.1 \mathrm{M}(15 \mathrm{~mL})$. The aqueous layers were combined and extracted with $\mathrm{Et}_{2} \mathrm{O}(20 \mathrm{~mL})$. The organic layers were combined, washed with saturated $\mathrm{NaHCO}_{3}(20 \mathrm{~mL})$, brine $(20 \mathrm{~mL})$, dried with $\mathrm{MgSO}_{4}$ and concentrated under reduced pressure. Purification by flash chromatography ( $\mathrm{PET}_{\mathrm{Et}} \mathrm{O}$ 8/2) afforded 7e (102 $\mathrm{mg}, 0.342 \mathrm{mmol}, 86 \%$ ) as brown solid. $\mathrm{R} f 0.4$ (PET/Et $2 \mathrm{O}$ 7:3, UV/Anisaldehyde). Mp 55-58 ${ }^{\circ} \mathrm{C} .{ }^{1} \mathrm{H}$ NMR $\left(\mathrm{CDCl}_{3}, 400 \mathrm{MHz}\right) \delta 8.11(\mathrm{br} \mathrm{s}, 1 \mathrm{H}, \mathrm{NH}), 7.79(\mathrm{~m}, 1 \mathrm{H}, \mathrm{ArH}), 7.40(\mathrm{~d}, J=2.7 \mathrm{~Hz}, 1 \mathrm{H}, \mathrm{ArH}), 7.36(\mathrm{~m}, 1 \mathrm{H}$, $\mathrm{ArH}), 7.26$ (m, $2 \mathrm{H}, \mathrm{ArH}), 1.22$ (m, $21 \mathrm{H}, \mathrm{TIPS}) .{ }^{13} \mathrm{C} \mathrm{NMR}\left(\mathrm{CDCl}_{3}, 100 \mathrm{MHz}\right) \delta 135.1,128.9,128.3,123.1$, 120.8, 120.1, 111.4, 100.4, 99.3, 92.19, 18.8, 11.5. IR v 3407 (m), 3062 (w), 2942 (s), 2891 (m), 2864 (s), 2152 (s), 1620 (w), 1532 (w), 1457 (s), 1416 (m), 1383 (w), 1341 (w), 1325 (m), 1239 (s), 1128 (m), 1071 (m), 996 (m), 910 (m), 883 (s), 774 (s), 742 (s), 676 (s), 658 (s), 628 (s). HRMS(ESI) calcd for $\mathrm{C}_{19} \mathrm{H}_{28} \mathrm{NSi}^{+}$ $(\mathrm{M}+\mathrm{H})$ 298.1991, found 298.2001.

\section{Triethyl((5-hexylthiophen-2-yl)ethynyl)silane (9b)}

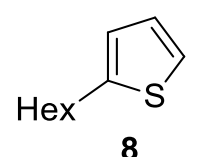

8

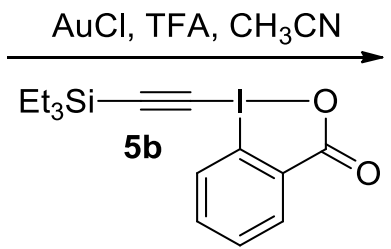

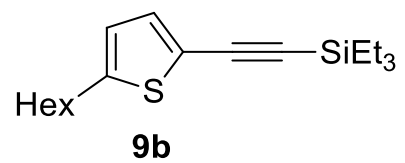

To a stirring solution of $\mathrm{AuCl}\left(4.6 \mathrm{mg}, 0.020 \mathrm{mmol}, 0.05\right.$ equiv) in $\mathrm{CH}_{3} \mathrm{CN} 1(2 \mathrm{~mL})$ was added 2hexylthiophene (8) $(72 \mu \mathrm{L}, 0.40 \mathrm{mmol}, 1$ equiv) under air. After $2 \mathrm{~min}$, TFA ( $36 \mu \mathrm{L}, 0.48 \mathrm{mmol}, 1.2$ equiv) and TES-EBX (5b) (186 mg, $0.480 \mathrm{mmol}, 1.2$ equiv) were added. The reaction was sealed and stirred at RT for $16 \mathrm{~h}$. $\mathrm{Et}_{2} \mathrm{O}(10 \mathrm{~mL})$ was added, the organic layer was washed twice with $0.1 \mathrm{M} \mathrm{NaOH}(15 \mathrm{~mL})$. The aqueous layers were combined and extracted with $\mathrm{Et}_{2} \mathrm{O}(20 \mathrm{~mL})$. The organic layers were combined, washed with saturated $\mathrm{NaHCO}_{3}(20 \mathrm{~mL})$, brine $(20 \mathrm{~mL})$, dried with $\mathrm{MgSO}_{4}$ and concentrated under reduced pressure. The resulting oil was purified by flash chromatography (pentane) to afford $9 \mathbf{b}$ (31 $\mathrm{mg}, 0.10 \mathrm{mmol}$, $25 \%)$ as a mixture with 2-hexylthiophene $(6 \mathrm{mg}, 0.04 \mathrm{mmol}, 9 \%$ recovered) as colorless oil. Analytical chromatography afforded pure compound. $\mathrm{R}_{\mathrm{f}}$ (pentane): 0.6. ${ }^{1} \mathrm{H} \mathrm{NMR}\left(400 \mathrm{MHz}, \mathrm{CDCl}_{3}\right) \delta 7.07(\mathrm{~d}, 1 \mathrm{H}, J=$ $3.6 \mathrm{~Hz}, \mathrm{ArH}), 6.63(\mathrm{dd}, 1 \mathrm{H}, J=3.6,0.8 \mathrm{~Hz}, \mathrm{ArH}), 2.78\left(\mathrm{t}, 2 \mathrm{H}, J=7.5 \mathrm{~Hz}, \mathrm{CH}_{2}\right), 1.66\left(\mathrm{~m}, 2 \mathrm{H}, \mathrm{CH}_{2}\right), 1.33$ $\left(\mathrm{m}, 6 \mathrm{H}, \mathrm{CH}_{2}\right), 1.05$ (t, $\left.9 \mathrm{H}, J=8.0 \mathrm{~Hz}, \mathrm{SiCH}_{2} \mathrm{CH}_{3}\right), 0.90\left(\mathrm{t}, 3 \mathrm{H}, J=6.8 \mathrm{~Hz}, \mathrm{CH}_{3}\right), 0.68(\mathrm{q}, 6 \mathrm{H}, J=7.9 \mathrm{~Hz}$, 
$\left.\mathrm{SiCH}_{2} \mathrm{CH}_{3}\right) .{ }^{13} \mathrm{C} \mathrm{NMR}\left(101 \mathrm{MHz}, \mathrm{CDCl}_{3}\right) \delta 148.2,132.6,123.9,120.6,99.2,95.3,31.5,30.2,29.7,28.7$, 22.5, 14.1, 7.5, 4.4. IR 2955 (s), 2931 (s), 2874 (m), 2856 (m), 2143 (s), 1727 (w), 1598 (w), $1535(\mathrm{w}), 1460$ (m), 1416 (w), 1374 (w), 1238 (w), 1167 (m), 1017 (m), 976 (w), 904 (w), 826 (w), 802 (m), 765 (s), 736 (s). HRMS (ESI) calcd for $\mathrm{C}_{18} \mathrm{H}_{30} \mathrm{AgSSi}^{+}[\mathrm{M}+\mathrm{Ag}]^{+} 413.0883$; found 413.0878 .

\section{tert-Butyl((5-hexylthiophen-2-yl)ethynyl)dimethylsilane (9c)}

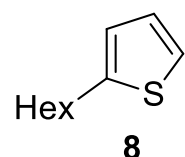

8

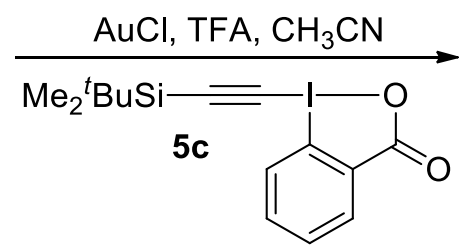

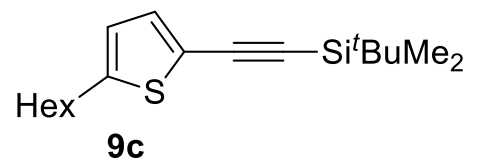

To a stirring solution of $\mathrm{AuCl}\left(4.6 \mathrm{mg}, 0.020 \mathrm{mmol}, 0.05\right.$ equiv) in $\mathrm{CH}_{3} \mathrm{CN} 1(2 \mathrm{~mL})$ was added 2hexylthiophene (8) $(72 \mu \mathrm{L}, 0.40 \mathrm{mmol}, 1$ equiv) under air. After $2 \mathrm{~min}$, TFA ( $36 \mu \mathrm{L}, 0.48 \mathrm{mmol}, 1.2$ equiv) and TBS-EBX (5c) (186 mg, $0.480 \mathrm{mmol}, 1.2$ equiv) were added. The reaction was sealed and stirred at RT for $14 \mathrm{~h}$. $\mathrm{Et}_{2} \mathrm{O}(10 \mathrm{~mL})$ was added, the organic layer was washed twice with $0.1 \mathrm{M} \mathrm{NaOH}(15 \mathrm{~mL})$. The aqueous layers were combined and extracted with $\mathrm{Et}_{2} \mathrm{O}(20 \mathrm{~mL})$. The organic layers were combined, washed with saturated $\mathrm{NaHCO}_{3}(20 \mathrm{~mL})$, brine $(20 \mathrm{~mL})$, dried with $\mathrm{MgSO}_{4}$ and concentrated under reduced pressure. The resulting oil was purified by flash chromatography (pentane) to afford 9c $(58 \mathrm{mg}, 0.19 \mathrm{mmol}$, $47 \%$ ) as colorless oil. $\mathrm{R}_{\mathrm{f}}$ (pentane): 0.6. ${ }^{1} \mathrm{H} \mathrm{NMR}\left(400 \mathrm{MHz}, \mathrm{CDCl}_{3}\right) \delta 7.04(\mathrm{~d}, 1 \mathrm{H}, J=3.5 \mathrm{~Hz}, \mathrm{ArH}), 6.61$ (d, $1 \mathrm{H}, J=3.2 \mathrm{~Hz}, \mathrm{ArH}), 2.76\left(\mathrm{t}, 2 \mathrm{H}, J=7.5 \mathrm{~Hz}, \mathrm{CH}_{2}\right), 1.63\left(\mathrm{~m}, 2 \mathrm{H}, \mathrm{CH}_{2}\right), 1.32\left(\mathrm{~m}, 6 \mathrm{H}, \mathrm{CH}_{2}\right), 0.98(\mathrm{~s}, 9$ $\mathrm{H}, t \mathrm{Bu}), 0.89\left(\mathrm{~m}, 3 \mathrm{H}, \mathrm{CH}_{3}\right), 0.17(\mathrm{~s}, 6 \mathrm{H}, \mathrm{Me}) .{ }^{13} \mathrm{C} \mathrm{NMR}\left(101 \mathrm{MHz}, \mathrm{CDCl}_{3}\right) \delta 148.3,132.6,123.9,120.5$, 98.7, 96.1, 31.5, 31.5, 30.2, 28.7, 26.1, 22.6, 16.8, 14.1, -4.6. IR 2955 (m), 2929 (m), 2856 (m), $2144(\mathrm{~m})$, 1535 (w), 1470 (w), 1362 (w), 1254 (w), 1168 (w), 1033 (w), 1008 (w), $941(w), 910(w), 827(\mathrm{~s}), 813$ (s), $776(\mathrm{~s}), 735$ (m), 677 (m). HRMS (ESI) calcd for $\mathrm{C}_{18} \mathrm{H}_{31} \mathrm{SSi}^{+}[\mathrm{M}+\mathrm{H}]^{+}$307.1910; found 307.1919.

\section{tert-Butyl((5-hexylthiophen-2-yl)ethynyl)diphenylsilane (9d)}
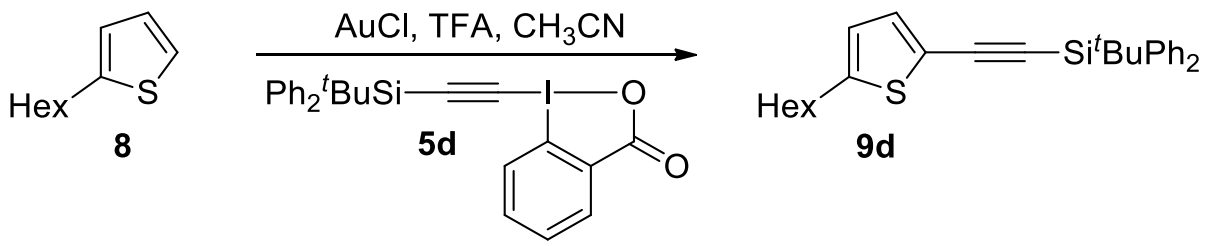

To a stirring solution of $\mathrm{AuCl}\left(4.6 \mathrm{mg}, 0.020 \mathrm{mmol}, 0.05\right.$ equiv) in $\mathrm{CH}_{3} \mathrm{CN} 1$ (2 mL) was added 2hexylthiophene (8) $(72 \mu \mathrm{L}, 0.40 \mathrm{mmol}, 1$ equiv) under air. After $2 \mathrm{~min}$, TFA (36 $\mu \mathrm{L}, 0.48 \mathrm{mmol}, 1.2$ equiv) and $5 \mathbf{d}$ (250 mg, $0.480 \mathrm{mmol}, 1.2$ equiv) were added. The reaction was sealed and stirred at RT for $14 \mathrm{~h}$. $\mathrm{Et}_{2} \mathrm{O}(10 \mathrm{~mL})$ was added, the organic layer was washed twice with $0.1 \mathrm{M} \mathrm{NaOH}(15 \mathrm{~mL})$. The aqueous layers were combined and extracted with $\mathrm{Et}_{2} \mathrm{O}(20 \mathrm{~mL})$. The organic layers were combined, washed with saturated $\mathrm{NaHCO}_{3}(20 \mathrm{~mL})$, brine $(20 \mathrm{~mL})$, dried with $\mathrm{MgSO}_{4}$ and concentrated under reduced pressure. The resulting oil was purified by flash chromatography (pentane) to afford 9d (122 $\mathrm{mg}, 0.280 \mathrm{mmol}, 71 \%$ ) as colorless oil. $\mathrm{R}_{\mathrm{f}}$ (pentane): 0.3. ${ }^{1} \mathrm{H}$ NMR $\left(400 \mathrm{MHz}, \mathrm{CDCl}_{3}\right) \delta$ 7.90-7.85 (m, $\left.4 \mathrm{H}, \mathrm{ArH}\right), 7.46-7.39(\mathrm{~m}, 6 \mathrm{H}$, ArH), $7.22(\mathrm{~d}, 1 \mathrm{H}, J=3.6 \mathrm{~Hz}, \mathrm{ArH}), 6.70(\mathrm{~d}, 1 \mathrm{H}, J=3.6 \mathrm{~Hz}, \mathrm{ArH}), 2.83\left(\mathrm{t}, 2 \mathrm{H}, J=7.6 \mathrm{~Hz}, \mathrm{CH}_{2}\right), 1.71(\mathrm{~m}$, $\left.2 \mathrm{H}, \mathrm{CH}_{2}\right), 1.37\left(\mathrm{~m}, 6 \mathrm{H}, \mathrm{CH}_{2}\right), 1.18(\mathrm{~s}, 9 \mathrm{H}, t \mathrm{Bu}), 0.94\left(\mathrm{~m}, 3 \mathrm{H}, \mathrm{CH}_{3}\right) .{ }^{13} \mathrm{C} \mathrm{NMR}\left(101 \mathrm{MHz}, \mathrm{CDCl}_{3}\right) \delta 148.9$, 135.6, 133.2, 133.2, 129.5, 127.7, 124.1, 120.3, 102.2, 93.0, 31.5, 31.5, 30.2, 28.7, 27.1, 22.5, 18.8, 14.1. IR $3047(\mathrm{w}), 2958(\mathrm{~m}), 2930(\mathrm{~m}), 2857(\mathrm{~m}), 2144(\mathrm{~m}), 1471(\mathrm{w}), 1429(\mathrm{~m}), 1362(\mathrm{w}), 1263(\mathrm{w}), 1168(\mathrm{w})$, $1110(\mathrm{~m}), 1031(\mathrm{w}), 1009$ (w), $905(\mathrm{~m}), 821(\mathrm{~m}), 803(\mathrm{~m}), 743(\mathrm{~m}), 736$ (s), 700 (s). HRMS (ESI) calcd for $\mathrm{C}_{28} \mathrm{H}_{35} \mathrm{SSi}^{+}[\mathrm{M}+\mathrm{H}]^{+}$431.2223; found 431.2231. 

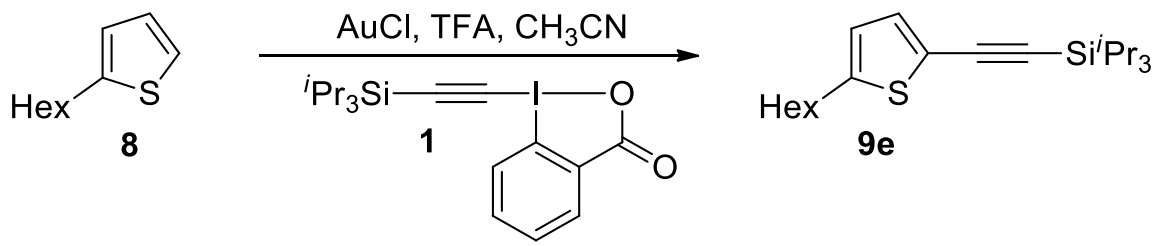

To a stirring solution of $\mathrm{AuCl}\left(4.6 \mathrm{mg}, 0.020 \mathrm{mmol}, 0.05\right.$ equiv) in $\mathrm{CH}_{3} \mathrm{CN} 1$ (2 mL) was added 2hexylthiophene (8) $(72 \mu \mathrm{L}, 0.40 \mathrm{mmol}, 1$ equiv) under air. After $2 \mathrm{~min}$, TFA (36 $\mu \mathrm{L}, 0.48 \mathrm{mmol}, 1.2$ equiv) and 1 (206 mg, $0.480 \mathrm{mmol}, 1.2$ equiv) were added. The reaction was sealed and stirred at RT for $14 \mathrm{~h}$. $\mathrm{Et}_{2} \mathrm{O}$ $(10 \mathrm{~mL})$ was added, the organic layer was washed twice with $0.1 \mathrm{M} \mathrm{NaOH}(15 \mathrm{~mL})$. The aqueous layers were combined and extracted with $\mathrm{Et}_{2} \mathrm{O}(20 \mathrm{~mL})$. The organic layers were combined, washed with saturated $\mathrm{NaHCO}_{3}(20 \mathrm{~mL})$, brine $(20 \mathrm{~mL})$, dried with $\mathrm{MgSO}_{4}$ and concentrated under reduced pressure. The resulting oil was purified by flash chromatography (pentane) to afford 9e (116 mg, $0.333 \mathrm{mmol}, 83 \%)$ as slightly yellow oil. Rf 0.6 (pentane, UV). ${ }^{1} \mathrm{H}$ NMR $\delta 7.08(\mathrm{~d}, J=3.5 \mathrm{~Hz}, 1 \mathrm{H}, \mathrm{ArH}), 6.65(\mathrm{~d}, J=3.5 \mathrm{~Hz}, 1 \mathrm{H}, \operatorname{ArH})$, $2.80\left(\mathrm{t}, J=7.5 \mathrm{~Hz}, 2 \mathrm{H}, \mathrm{CH}_{2}\right), 1.68\left(\mathrm{~m}, \mathrm{CH}_{2}\right), 1.44-1.30\left(\mathrm{~m}, 6 \mathrm{H}, \mathrm{CH}_{2}\right), 1.15(\mathrm{~m}, 21 \mathrm{H}$, TIPS), $0.93(\mathrm{t}, J=6.1$ $\left.\mathrm{Hz}, 3 \mathrm{H}, \mathrm{CH}_{3}\right) .{ }^{13} \mathrm{C} \mathrm{NMR} \delta 148.1,132.4,123.9,121.0,99.8,94.3,31.7,31.6,30.2,28.7,22.6,18.7,14.1$, 11.4. IR ( $\left.\mathrm{cm}^{-1}\right)$ : 2958 (s), 2928 (s), 2865 (s), 2143 (s), 1535 (w), 1463 (s), 1382 (w), 1367 (w), 1243 (w), 1167 (m), 1074 (w), 1018 (m), 997 (m), 920 (w), 883 (s), 800 (s), 757 (s), 736 (s), 678 (s), 658 (s), 633 (s). HRMS(ESI) calcd for $\mathrm{C}_{21} \mathrm{H}_{37} \mathrm{SSi}^{+}(\mathrm{M}+\mathrm{H}) 349.2385$, found 349.2381.

\section{Aromatic and alkylic alkynyl benziodoxolones}

\section{1-[Phenylethynyl]-1,2-benziodoxol-3(1H)-one (Ph-EBX, 5e)}
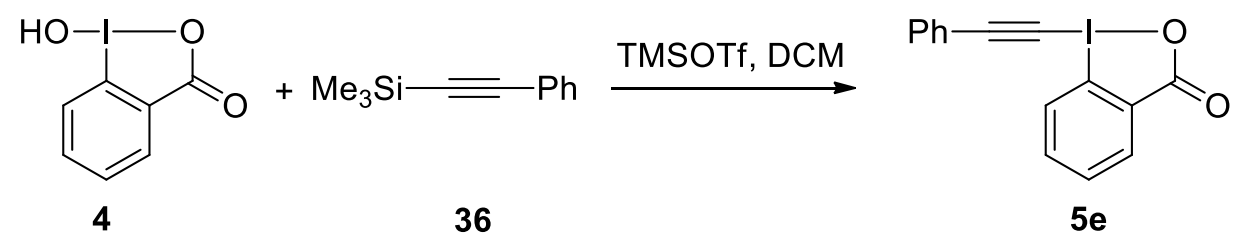

Trimethylsilyl triflate $(7.50 \mathrm{~mL}, 41.5 \mathrm{mmol}, 1.1$ equiv) was added to a suspension of 2-iodosylbenzoic acid (10) (10.0 g, $37.7 \mathrm{mmol}, 1$ equiv) in $\mathrm{CH}_{2} \mathrm{Cl}_{2}(100 \mathrm{~mL})$ at $\mathrm{RT}$. The resulting yellow mixture was stirred for $1 \mathrm{~h}$, followed by the dropwise addition of trimethyl(phenylethynyl)silane (36) $(8.10 \mathrm{~mL}, 41.5 \mathrm{mmol}, 1.1$ equiv) (slightly exothermic). The resulting suspension was stirred for $6 \mathrm{~h}$ at RT, during this time a white solid was formed. A saturated solution of $\mathrm{NaHCO}_{3}(100 \mathrm{~mL})$ was then added and the mixture was stirred vigorously. The resulting suspension was filtered on a glass filter of porosity 4 . The two layers of the mother liquors were separated and the organic layer was washed with sat. $\mathrm{NaHCO}_{3}(100 \mathrm{~mL})$, dried over $\mathrm{MgSO}_{4}$, filtered and evaporated under reduced pressure. The resulting mixture was combined with the solid obtained by filtration and boiled in $\mathrm{CH}_{3} \mathrm{CN}(300 \mathrm{~mL})$. The mixture was cooled down, filtered and dried under high vacuum to afford 5e $(6.08 \mathrm{~g}, 17.4 \mathrm{mmol}, 46 \%)$ as a colorless solid. $\mathrm{Mp}$ (Dec.) $155-160^{\circ} \mathrm{C}$ (lit $153-155^{\circ} \mathrm{C}$ ). ${ }^{1} \mathrm{H} \mathrm{NMR}\left(400 \mathrm{MHz}, \mathrm{CDCl}_{3}\right)(\mathrm{ca} 0.03 \mathrm{mmol} / \mathrm{ml}) \delta 8.46(\mathrm{~m}, 1 \mathrm{H}, \mathrm{ArH}), 8.28(\mathrm{~m}, 1 \mathrm{H}, \mathrm{ArH}), 7.80(\mathrm{~m}, 2 \mathrm{H}$, $\mathrm{ArH}), 7.63(\mathrm{~m}, 2 \mathrm{H}, \mathrm{ArH}), 7.48(\mathrm{~m}, 3 \mathrm{H}, \mathrm{ArH}) .{ }^{13} \mathrm{C} \mathrm{NMR}\left(101 \mathrm{MHz}, \mathrm{CDCl}_{3}\right) \delta 163.9,134.9,132.9,132.5$, 131.6, 131.3. 130.8, 128.8, 126.2, 120.5, 116.2, 106.6, 50.2. Consistent with reported data. ${ }^{5}$ The crystal structure of 5d has been deposited at the Cambridge Crystallographic Data Centre and allocated the deposition number: CCDC 863351. 


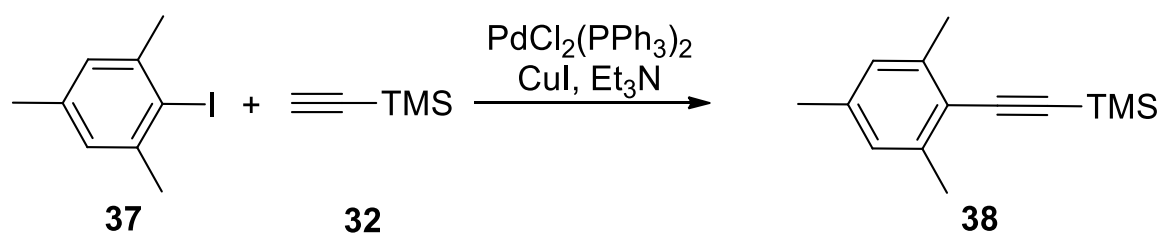

4-Iodoanisole (37) (1.05 g, $4.27 \mathrm{mmol}, 1$ equiv) was dissolved in $\mathrm{Et}_{3} \mathrm{~N}$ (10 mL) (without prior drying). After three freeze-thraw-pump cycle, $\mathrm{PdCl}_{2}\left(\mathrm{PPh}_{3}\right)_{2}(30 \mathrm{mg}, 0.42 \mathrm{mmol}, 0.1$ equiv) and $\mathrm{CuI}(16 \mathrm{mg}, 0.84 \mathrm{mmol}$, 0.2 equiv) were added under $\mathrm{N}_{2}$. After the addition of trimethylsilylacetylene (32) $(1.2 \mathrm{~mL}, 8.5 \mathrm{mmol}, 2$ equiv), the green suspension was stirred at RT for $1 \mathrm{~h}$. The reaction mixture was reduced under vacuum, dissolved in $\mathrm{CH}_{2} \mathrm{Cl}_{2}(30 \mathrm{~mL})$, washed with $5 \%$ EDTA solution $(30 \mathrm{~mL})$ and water $(30 \mathrm{~mL})$. The organic layers were them dried over $\mathrm{MgSO}_{4}$, filtered and reduced under vacuum. The resulting oil was purified by column chromatography (PET) to afford $38(526 \mathrm{mg}, 2.43 \mathrm{mmol}, 66 \%)$ along with $15 \%$ of starting material. $\mathrm{R}_{\mathrm{f}} 0.5$ (PET). ${ }^{1} \mathrm{H}$ NMR (400 MHz, $\left.\mathrm{CDCl}_{3}\right) \delta 6.87$ (s, $\left.2 \mathrm{H}, \mathrm{ArH}\right), 2.41\left(\mathrm{~s}, 6 \mathrm{H}, \mathrm{CH}_{3}\right), 2.29\left(\mathrm{~s}, 3 \mathrm{H}, \mathrm{CH}_{3}\right), 0.28$ (s, $9 \mathrm{H}, \mathrm{TMS}$ ). Used without further purification.

\section{1-[2,4,6-Trimethylphenylethynyl]-1,2-benziodoxol-3(1H)-one (Mes-EBX, 5f)}

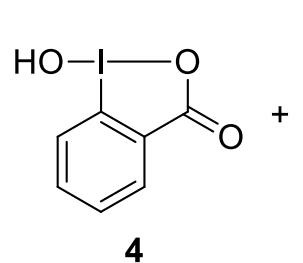

4

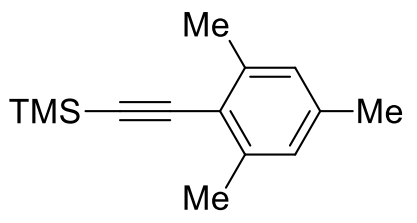

38

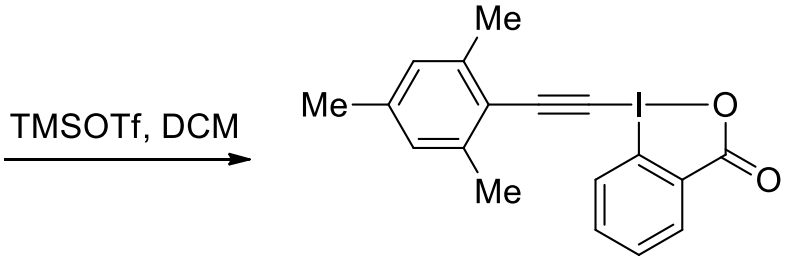

$5 f$

Trimethylsilyl triflate $(212 \mu \mathrm{L}, 1.15 \mathrm{mmol}, 1.1$ equiv) was added to a suspension of 2-iodosylbenzoic acid (4) (1.00 g, $1.05 \mathrm{mmol}, 1$ equiv) in $\mathrm{CH}_{2} \mathrm{Cl}_{2}(4 \mathrm{~mL})$ at $\mathrm{RT}$. The resulting yellow mixture was stirred for 1 $\mathrm{h}$, followed by the dropwise addition of (mesitylethynyl)trimethylsilane (38) (250 mg, $1.15 \mathrm{mmol}, 1.1$ equiv) dissolved in $\mathrm{CH}_{2} \mathrm{Cl}_{2}(1 \mathrm{~mL})$. The resulting suspension was stirred for $6 \mathrm{~h}$ at $\mathrm{RT}$. A saturated solution of $\mathrm{NaHCO}_{3}(5 \mathrm{~mL})$ was then added and the mixture was stirred vigorously. The layers were separated and the organic layer was washed with sat. $\mathrm{NaHCO}_{3}(10 \mathrm{~mL})$, dried over $\mathrm{MgSO}_{4}$, filtered and evaporated under reduced pressure. The resulting solid was recrystallized in $\mathrm{CH}_{3} \mathrm{CN}$ (ca $20 \mathrm{ml}$ ). The mother liquors were concentrated and and the obtained solid recrystallized in $\mathrm{CH}_{3} \mathrm{CN}(4 \mathrm{~mL})$. Both solids were combined, washed with pentane and dried under high vacuum to afford $\mathbf{5 f}(120 \mathrm{mg}, 0.307 \mathrm{mmol}, 30 \%)$ as a tan solid. $\mathrm{Mp}$ (Dec.) $171-175^{\circ} \mathrm{C} .{ }^{1} \mathrm{H} \mathrm{NMR}\left(400 \mathrm{MHz}, \mathrm{CDCl}_{3}\right)(\mathrm{ca} 0.01 \mathrm{mmol} / \mathrm{ml}) \delta 8.38(\mathrm{~m}, 1 \mathrm{H}, \mathrm{ArH}), 8.28(\mathrm{~m}, 1 \mathrm{H}$, ArH), 7.72 (m, $2 \mathrm{H}, \mathrm{ArH}), 6.92$ (s, $2 \mathrm{H}, \mathrm{MesH}), 2.45\left(\mathrm{~s}, 6 \mathrm{H}, \mathrm{CH}_{3}\right), 2.31\left(\mathrm{~s}, 3 \mathrm{H}, \mathrm{CH}_{3}\right) .{ }^{13} \mathrm{C} \mathrm{NMR}(101 \mathrm{MHz}$, $\left.\mathrm{CDCl}_{3}\right) \delta 166.7,142.1,140.5,134.5,132.2,131.5,131.3,128.0,126.2,117.5,116.5,105.1,55.6,21.4,21.0$. IR 2979 (w), $2916(w), 2247(w), 2131(w), 1650(\mathrm{~m}), 1623$ (m), $1562(w), 1439(w), 1333(w), 1292(w)$, $1212(\mathrm{w}), 1146$ (w), 1008 (w), 906 (s), 855 (w), 833 (w), 729 (s), 647 (m). HRMS(ESI) calcd for $\mathrm{C}_{18} \mathrm{H}_{16} \mathrm{O}_{2} \mathrm{I}^{+}$ $(\mathrm{M}+\mathrm{H})$ 391.0195, found 391.0191.

\section{((4-Nitrophenyl)ethynyl)trimethylsilane (40)}

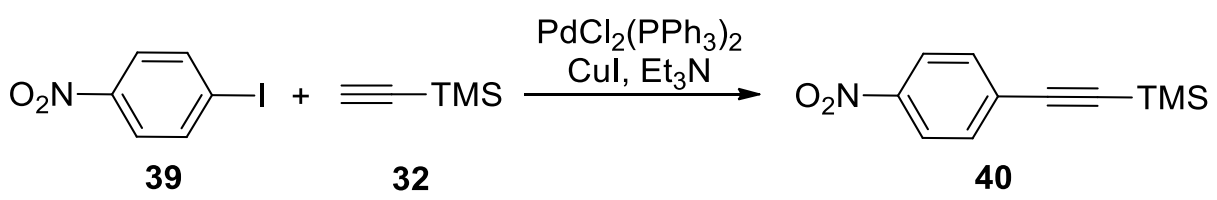


Following a slight modification of the reported procedure, ${ }^{8}$ 1-iodo-4-nitrobenzene (39) (1.06 g, 4.27 mmol, 1 equiv) was dissolved in $\mathrm{Et}_{3} \mathrm{~N}(10 \mathrm{~mL})$ (without prior drying). After three freeze-thaw-pump cycles, $\mathrm{PdCl}_{2}\left(\mathrm{PPh}_{3}\right)_{2}$ (30 mg, $0.42 \mathrm{mmol}, 0.1$ equiv) and $\mathrm{CuI}$ (16 mg, $0.84 \mathrm{mmol}, 0.2$ equiv) were added under $\mathrm{N}_{2}$. After the addition of trimethylsilylacetylene (32) $(1.2 \mathrm{~mL}, 8.5 \mathrm{mmol}, 2$ equiv), the green suspension was stirred at RT for $1 \mathrm{~h}$. The reaction mixture was reduced under vacuum, dissolved in $\mathrm{CH}_{2} \mathrm{Cl}_{2}(30 \mathrm{~mL})$, washed with $5 \%$ EDTA solution $(30 \mathrm{~mL})$ and water $(30 \mathrm{~mL})$. The organic layers were them dried over $\mathrm{MgSO}_{4}$, filtered and reduced under vacuum. The resulting oil was purified by column chromatography (PET/EtOAc 99/1) to afford 40 (890 mg, $4.06 \mathrm{mmol}, 95 \%) . \mathrm{R}_{\mathrm{f}} 0.3$ (PET/EtOAc 99/1). ${ }^{1} \mathrm{H}$ NMR (400 MHz, $\left.\mathrm{CDCl}_{3}\right) \delta 8.17(\mathrm{~d}, 2 \mathrm{H}, J=8.6,2 \mathrm{~Hz}, \mathrm{ArH}), 7.59(\mathrm{~d}, 2 \mathrm{H}, J=8.6 \mathrm{~Hz}, \mathrm{ArH}), 0.27$ (s, $\left.9 \mathrm{H}, \mathrm{TMS}\right) .{ }^{13} \mathrm{C}$ NMR $\left(101 \mathrm{MHz}, \mathrm{CDCl}_{3}\right) \delta 147.1,132.7,130.0,123.5,102.7,100.6,-0.3$.The characterization data for compound 40 corresponded to the reported values. ${ }^{8}$

\section{1-[4-Nitrophenylethynyl]-1,2-benziodoxol-3(1H)-one (4-NO2Ph-EBX, 5g)}<smiles>O=C1OI(O)c2ccccc21</smiles>

4

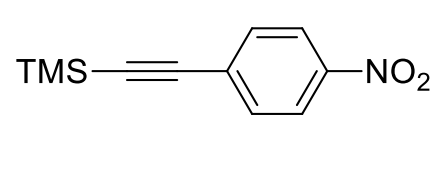

40

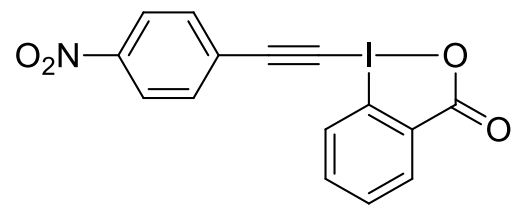

$5 \mathrm{~g}$

Trimethylsilyl triflate ( $763 \mu \mathrm{L}, 4.15 \mathrm{mmol}, 1.1$ equiv) was added to a suspension of 2-iodosylbenzoic acid (4) (1.00 g, $3.77 \mathrm{mmol}, 1$ equiv) in $\mathrm{CH}_{2} \mathrm{Cl}_{2}(10 \mathrm{~mL})$ at $\mathrm{RT}$. The resulting yellow mixture was stirred for $1 \mathrm{~h}$, followed by the dropwise addition of ((4-nitrophenyl)ethynyl)trimethylsilane (40) (1.01 g, $4.15 \mathrm{mmol}$, 1.1 equiv). The resulting suspension was stirred for $6 \mathrm{~h}$ at RT. A saturated solution of $\mathrm{NaHCO}_{3}(15 \mathrm{~mL})$ was then added and the mixture was stirred vigorously. The resulting suspension was filtered, washed with acetone and dried under vacuum to afford $\mathbf{5 g}(864 \mathrm{mg}, 2.19 \mathrm{mmol}, 59 \%)$ as a pale brown solid. Mp (Dec.) $142-151^{\circ} \mathrm{C} .{ }^{1} \mathrm{H}$ NMR $\left(400 \mathrm{MHz}, \mathrm{CDCl}_{3}\right)(c a 0.01 \mathrm{mmol} / \mathrm{ml}) \delta 8.44(\mathrm{~m}, 1 \mathrm{H}, \mathrm{ArH}), 8.31(\mathrm{~m}, 2 \mathrm{H}, \mathrm{ArH})$, $8.23(\mathrm{~m}, 1 \mathrm{H}, \mathrm{ArH}), 7.86-7.74(\mathrm{~m}, 4 \mathrm{H}, \mathrm{ArH}) .{ }^{13} \mathrm{C} \mathrm{NMR}\left(101 \mathrm{MHz}, \mathrm{CDCl}_{3}\right) \delta 166.3,148.5,135.2,133.6$, 132.7, 131.9, 131.1, 127.1, 126.2, 123.9, 116.0, 103.2, 57.1. IR 3104 (w), $2432(w), 2164(w), 1938(w)$, 1614 (s), 1586 (m), 1515 (s), 1403 (m), 1341 (s), 1305 (s), 1221 (w), 1105 (w), 1008 (w), 858 (s), 828 (m), $740(\mathrm{~s}), 689(\mathrm{~m}), 636(\mathrm{~m})$. HRMS(ESI) calcd for $\mathrm{C}_{15} \mathrm{H}_{9} \mathrm{NO}_{4} \mathrm{I}^{+}(\mathrm{M}+\mathrm{H})$ 393.9576, found 393.9577 .

\section{1-[Butyl]-1,2-benziodoxol-3(1H)-one (5h)}
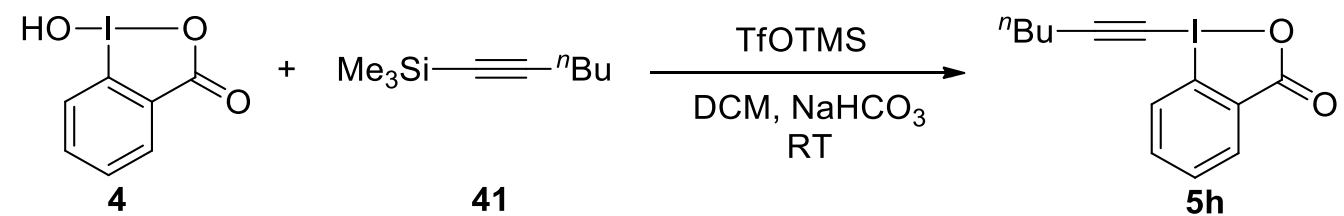

Trimethylsilyltriflate $(0.595 \mathrm{~mL}, 3.23 \mathrm{mmol}, 1.0$ equiv, freshly distilled $)$ was added dropwise to a stirred solution of 2-iodosylbenzoic acid (4) $\left(0.78 \mathrm{~g}, 2.9 \mathrm{mmol}, 1\right.$ equiv) in $\mathrm{CH}_{2} \mathrm{Cl}_{2}(10 \mathrm{~mL})$ at $0^{\circ} \mathrm{C}$. After $1 \mathrm{~h}$ at $\mathrm{RT}$, (trimethylsilyl) $\left({ }^{n}\right.$ butyl)acetylene (41) $(0.50 \mathrm{~g}, 3.2 \mathrm{mmol}, 1.1 \mathrm{equiv})$ was added dropwise. After $5 \mathrm{~h}$, a saturated solution of $\mathrm{NaHCO}_{3}(10 \mathrm{~mL})$ was added. The layers were separated and the aqueous layer was extracted with $\mathrm{CH}_{2} \mathrm{Cl}_{2}(10 \mathrm{~mL})$. The organic layers were combined, washed with $\mathrm{NaHCO}_{3}(20 \mathrm{~mL})$, dried over $\mathrm{MgSO}_{4}$, filtered and concentrated. Purification by column chromatography (EtOAc) afforded $\mathbf{5 h}$ (261 $\mathrm{mg}, 0.795 \mathrm{mmol}, 28 \%)$ as yellow oil. $\mathrm{R}_{\mathrm{f}}(\mathrm{EtOAc}): 0.2 .{ }^{1} \mathrm{H} \mathrm{NMR}\left(400 \mathrm{MHz}, \mathrm{CDCl}_{3}\right) \delta 8.40(\mathrm{~m}, 1 \mathrm{H}), 8.17$

\footnotetext{
${ }^{8}$ Sakai, N.; Annaka, K.; Konakahara, T. Org. Lett. 2004, 6, 1527
} 
(m, $1 \mathrm{H}), 7.75(\mathrm{~m}, 2 \mathrm{H}), 2.60(\mathrm{t}, 2 \mathrm{H}, J=7.1 \mathrm{~Hz}), 1.64(\mathrm{~m}, 2 \mathrm{H}), 1.49(\mathrm{~m}, 2 \mathrm{H}), 0.97(\mathrm{t}, 3 \mathrm{H}, J=7.3 \mathrm{~Hz}) .{ }^{13} \mathrm{C}$ NMR $\left(101 \mathrm{MHz}, \mathrm{CDCl}_{3}\right) \delta 166.5,134.6,132.4,131.5,131.5,126.1,115.5,109.7,39.2,30.2,22.0,20.1$, 13.5. IR 3445 (w), 3081 (w), 2959 (w), 2929 (w), 2863 (w), 1610 (s), 1586 (m), 1560 (m), 1459 (w), 1439 (m), 1350 (m), 1249 (s), 1224 (m), 1154 (s), 1030 (s), 1006 (w), 946 (w), 836 (m), 745 (s), 690 (m), 637 (s). HRMS (ESI) calcd for $\mathrm{C}_{13} \mathrm{H}_{14} \mathrm{IO}_{2}{ }^{+}[\mathrm{M}+\mathrm{H}]^{+} 329.0033$; found 329.0034 .

\section{(3,3-dimethylbut-1-yn-1-yl)trimethylsilane (43)}
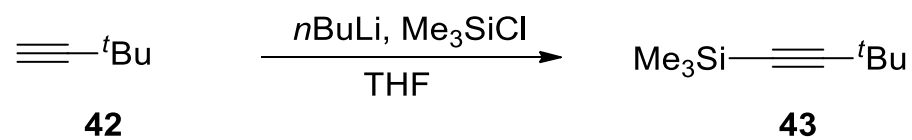

43

Following a reported procedure, ${ }^{9} n$-butyllithium $(2.5 \mathrm{M}$ in hexanes, $8.3 \mathrm{~mL}, 21 \mathrm{mmol}, 1.04$ equiv) was added dropwise to a stirred solution of 3,3-dimethylbut-1-yne (42) $(2.54 \mathrm{~mL}, 20.4 \mathrm{mmol}, 1.02$ equiv) in THF (70 $\mathrm{mL})$ at $-78{ }^{\circ} \mathrm{C}$. The mixture was stirred for $2 \mathrm{~h}$ at $-78^{\circ} \mathrm{C}$. Trimethylsilylchloride $(2.54 \mathrm{~mL}, 20.0 \mathrm{mmol}, 1.0$ equiv) dissolved in THF $(10 \mathrm{~mL})$ was added dropwise. The mixture was then allowed to warm to room temperature and stirred overnight. A saturated solution of ammonium chloride $(100 \mathrm{~mL})$ was added, and the reaction mixture was extracted with $\mathrm{CH}_{2} \mathrm{Cl}_{2}(2 \times 100 \mathrm{~mL})$. The organic layer was washed with water and brine, then dried over $\mathrm{MgSO}_{4}$, filtered and concentrated under reduced pressure to obtain a colorless liquid which was further purified by Kugelrohr distillation $\left(\mathrm{bp}=50^{\circ} \mathrm{C}, \mathrm{p}=0.5 \mathrm{mbar}\right.$ ) to yield 43 (1.35 g, 8.75 mmol, $44 \%$ yield) as a colorless liquid. ${ }^{1} \mathrm{H}$ NMR $\left(400 \mathrm{MHz}, \mathrm{CDCl}_{3}\right) \delta 1.21\left(\mathrm{~s}, 9 \mathrm{H},{ }^{t} \mathrm{Bu}\right), 0.13(\mathrm{~s}, 9 \mathrm{H}, \mathrm{TMS})$. The characterization data for compound $\mathbf{4 3}$ corresponded to the reported values. ${ }^{9}$

\section{1-[3,3-Dimethylbutynyl]-1,2-benziodoxol-3(1H)-one (5i)}<smiles>O=C1OI(O)c2ccccc21</smiles>

4

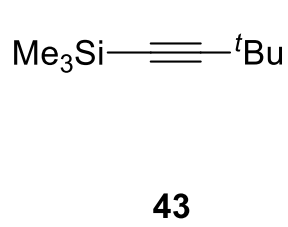

43

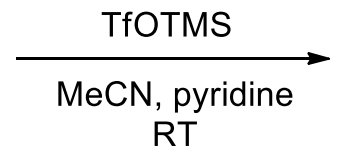
RT

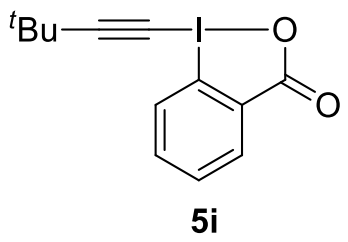

$5 \mathbf{i}$

Following a reported procedure, ${ }^{5}$ trimethylsilyltriflate $(1.52 \mathrm{~mL}, 8.42 \mathrm{mmol}, 1.0$ equiv, freshly distilled) was added dropwise to a stirred solution of 2-iodosylbenzoic acid (4) (2.69 g, $10.1 \mathrm{mmol}, 1.2$ equiv) in acetonitrile (30 mL). 3,3-dimethylbut-1-yn-1-yl)trimethylsilane (43) (1.30 g, 8.42 mmol, 1.0 equiv) was then added dropwise, followed, after $15 \mathrm{~min}$, by the addition of pyridine ( $680 \mu \mathrm{L}, 8.42 \mathrm{mmol}, 1.0$ equiv). The mixture was stirred $10 \mathrm{~min}$. The solvent was then removed under reduced pressure. $\mathrm{CH}_{2} \mathrm{Cl}_{2}$ and $1 \mathrm{M} \mathrm{NaOH}$ were added. The resulting suspension was filtered. The layers were separated and the aqueous layer was extracted with $\mathrm{CH}_{2} \mathrm{Cl}_{2}$. The organic layers were combined, dried over $\mathrm{MgSO}_{4}$, filtered and the solvent was evaporated under reduced pressure. Two recrystallization (with hot filtration) from acetonitrile were necessary to afforded $\mathbf{5 i}(1.43 \mathrm{~g}, 4.36 \mathrm{mmol}, 57 \%)$ as a colorless solid. ${ }^{10} \mathrm{Mp}$ (Dec.) $189-192{ }^{\circ} \mathrm{C} .{ }^{1} \mathrm{H}$ NMR $\left(400 \mathrm{MHz}, \mathrm{CDCl}_{3}\right)(\mathrm{ca} 0.06 \mathrm{mmol} / \mathrm{mL}) \delta 8.39(\mathrm{~m}, 1 \mathrm{H}, \mathrm{ArH}), 8.12(\mathrm{~m}, 1 \mathrm{H}, \mathrm{ArH}), 7.75(\mathrm{~m}, 2 \mathrm{H}, \mathrm{ArH}), 1.37$ $\left(\mathrm{s}, 9 \mathrm{H},{ }^{t} \mathrm{Bu}\right) .{ }^{13} \mathrm{C} \mathrm{NMR}\left(101 \mathrm{MHz}, \mathrm{CDCl}_{3}\right) \delta 166.5,134.6,132.4,131.5,131.4,125.8,117.5,115.5,38.2$, 30.6, 29.6. IR 3073 (w), 2971 (w), 2929 (w), 2868 (w), 2171 (w), 2140 (w), 1623 (s), $1561(w), 1440$ (w), $1330(\mathrm{~m}), 1297(\mathrm{~m}), 1248(\mathrm{~m}), 1006(\mathrm{w}), 911(\mathrm{w}), 832(\mathrm{~m}), 745$ (s), 690 (m), 652 (w). HRMS(ESI) calcd for $\mathrm{C}_{13} \mathrm{H}_{14} \mathrm{O}_{2} \mathrm{I}^{+}(\mathrm{M}+\mathrm{H}) 329.0038$, found 329.0026 .

\footnotetext{
${ }^{9}$ Earl, R. A.; Vollhardt, K. P. C. J. Org. Chem. 1984, 49, 4786.

${ }^{10}$ The difficult purification is certainly due to the excess of 2-iodosylbenzoic acid.
} 


\section{Reactions with aromatic and alkyl alkynyl benziodoxolones}

\section{3-(3,3-Dimethylbut-1-yn-1-yl)-1H-indole (10c)}

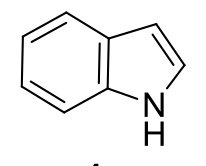

4
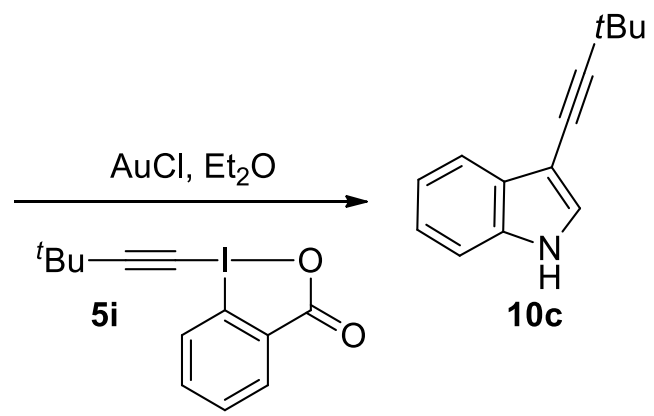

5i (79 mg, $0.24 \mathrm{mmol}, 1.2$ equiv) was added to a stirring solution of $\mathrm{AuCl}$ ( $2.3 \mathrm{mg}, 0.010 \mathrm{mmol}, 0.05$ equiv) and indole (4) (23.4 mg, $0.200 \mathrm{mmol}, 1.0$ equiv) in $\mathrm{Et}_{2} \mathrm{O} 1(4 \mathrm{~mL})$ under air. The reaction was sealed and stirred at room temperature for $16 \mathrm{~h}$. $\mathrm{Et}_{2} \mathrm{O}(10 \mathrm{~mL})$ was added, the organic layer was washed twice with $0.1 \mathrm{M} \mathrm{NaOH}(15 \mathrm{~mL})$. The aqueous layers were combined and extracted with $\mathrm{Et}_{2} \mathrm{O}(20 \mathrm{~mL})$. The organic layers were combined, washed with saturated $\mathrm{NaHCO}_{3}(20 \mathrm{~mL})$, brine $(20 \mathrm{~mL})$, dried with $\mathrm{MgSO}_{4}$ and concentrated under reduced pressure. Purification by flash chromatography (PET/EtOAc 95/5) afforded 10c (10 mg, $0.051 \mathrm{mmol}, 25 \%)$ as amorphous colorless solid. $\mathrm{R}_{f} 0.1$ (PET/EtOAc 95/5, UV). ${ }^{1} \mathrm{H}$ NMR (400 $\mathrm{MHz}_{\mathrm{CDCl}}$ ) $\delta 8.05$ (br s, $\left.1 \mathrm{H}, \mathrm{NH}\right), 7.72(\mathrm{~m}, 1 \mathrm{H}, \mathrm{ArH}), 7.34(\mathrm{~m}, 1 \mathrm{H}, \mathrm{ArH}), 7.31(\mathrm{~d}, 1 \mathrm{H}, J=2.6 \mathrm{~Hz}$, $\mathrm{ArH}), 7.21(\mathrm{~m}, 2 \mathrm{H}, \mathrm{ArH}), 1.39$ (s, $9 \mathrm{H}) .{ }^{13} \mathrm{C} \mathrm{NMR}\left(101 \mathrm{MHz}, \mathrm{CDCl}_{3}\right) \delta$ 135.1, 128.7, 126.6, 122.8, 120.3, 119.9, 111.1, 100.2, 99.4, 71.4, 31.4, 18.8. IR 3406 (m), 3050 (w), 2967 (m), $2925(\mathrm{w}), 2871(\mathrm{w}), 2133(\mathrm{w})$, $1535(\mathrm{w}), 1456(\mathrm{~m}), 1416(\mathrm{~m}), 1361(\mathrm{w}), 1328(\mathrm{~m}), 1259(\mathrm{~m}), 1238(\mathrm{w}), 1204(\mathrm{w}), 1128(\mathrm{w}), 1098(\mathrm{~m})$, $1076(w), 1008$ (w), 897 (w), 816 (w), 743 (s). HRMS (ESI) calcd for $\mathrm{C}_{14} \mathrm{H}_{16} \mathrm{~N}^{+}[\mathrm{M}+\mathrm{H}]^{+}$198.1277; found 198.1274.

\section{2-Hexyl-5-(phenylethynyl)thiophene (11a)}
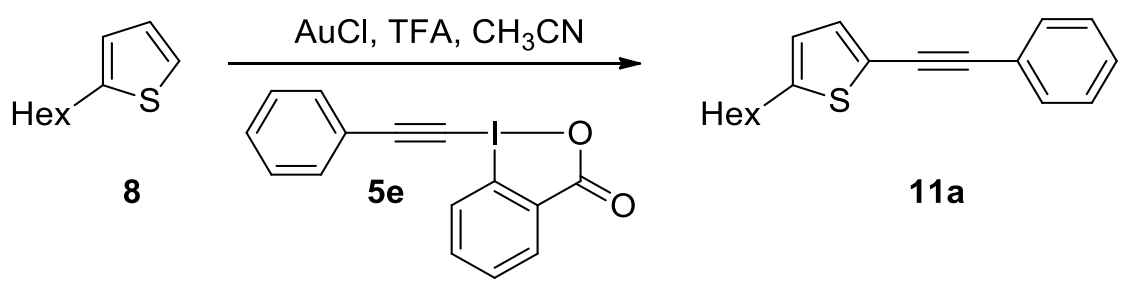

To a stirring solution of $\mathrm{AuCl}\left(4.6 \mathrm{mg}, 0.020 \mathrm{mmol}, 0.05\right.$ equiv) in $\mathrm{CH}_{3} \mathrm{CN}^{1}(2 \mathrm{~mL})$ was added 2hexylthiophene (8) $(72 \mu \mathrm{L}, 0.40 \mathrm{mmol}, 1$ equiv) under air. After $2 \mathrm{~min}$, TFA (36 $\mu \mathrm{L}, 0.48 \mathrm{mmol}, 1.2$ equiv) and $\mathrm{Ph}-\mathrm{EBX}$ (5e) (167 mg, $0.480 \mathrm{mmol}, 1.2$ equiv) were added. The reaction was sealed and stirred at RT for $14 \mathrm{~h}$. $\mathrm{Et}_{2} \mathrm{O}(10 \mathrm{~mL})$ was added, the organic layer was washed twice with $0.1 \mathrm{M} \mathrm{NaOH}(15 \mathrm{~mL})$. The aqueous layers were combined and extracted with $\mathrm{Et}_{2} \mathrm{O}(20 \mathrm{~mL})$. The organic layers were combined, washed with saturated $\mathrm{NaHCO}_{3}(20 \mathrm{~mL})$, brine $(20 \mathrm{~mL})$, dried with $\mathrm{MgSO}_{4}$ and concentrated under reduced pressure. The resulting oil was purified by flash chromatography (pentane) to afford 11a (25 mg, 0.093 mmol, $23 \%$ ) as colorless oil. $\mathrm{R}_{\mathrm{f}}$ (pentane): 0.4. ${ }^{1} \mathrm{H}$ NMR $\left(400 \mathrm{MHz}, \mathrm{CDCl}_{3}\right) \delta 7.49(\mathrm{~m}, 2 \mathrm{H}, \mathrm{ArH}), 7.33(\mathrm{~m}$, $3 \mathrm{H}$, , ArH), $7.10(\mathrm{~d}, 1 \mathrm{H}, J=3.6 \mathrm{~Hz}, \mathrm{ArH}), 6.67$ (d, $1 \mathrm{H}, J=3.6 \mathrm{~Hz}, \mathrm{ArH}), 2.80\left(\mathrm{t}, 2 \mathrm{H}, J=7.5 \mathrm{~Hz}, \mathrm{CH}_{2}\right.$ ), $1.67\left(\mathrm{~m}, 2 \mathrm{H}, \mathrm{CH}_{2}\right), 1.42-1.19\left(\mathrm{~m}, 6 \mathrm{H}, \mathrm{CH}_{2}\right), 0.89\left(\mathrm{~m}, 3 \mathrm{H}, \mathrm{CH}_{3}\right) .{ }^{13} \mathrm{C} \mathrm{NMR}\left(101 \mathrm{MHz}, \mathrm{CDCl}_{3}\right) \delta 148.4$, $131.9,131.3,128.3,128.1,124.2,123.2,120.4,92.2,83.1,31.5,31.5,30.2,28.7,22.6,14.1$. IR $3058(w)$, 3025 (w), 2955 (m), 2928 (s), 2855 (m), 2366 (w), 2205 (w), 1749 (w), 1598 (w), 1542 (w), 1493 (w), 1462 
(m), 1443 (w), $1378(\mathrm{w}), 1278(\mathrm{w}), 1212(\mathrm{w}), 1181(\mathrm{w}), 1114(\mathrm{w}), 1070(\mathrm{w}), 1025(\mathrm{w}), 914(\mathrm{w}), 805(\mathrm{~m})$, 755 (s), 690 (s), $673(\mathrm{~m})$. HRMS (ESI) calcd for $\mathrm{C}_{18} \mathrm{H}_{21} \mathrm{~S}^{+}[\mathrm{M}+\mathrm{H}]^{+}$269.1358; found 269.1349.

\section{2-Hexyl-5-(mesitylethynyl)thiophene (11b)}
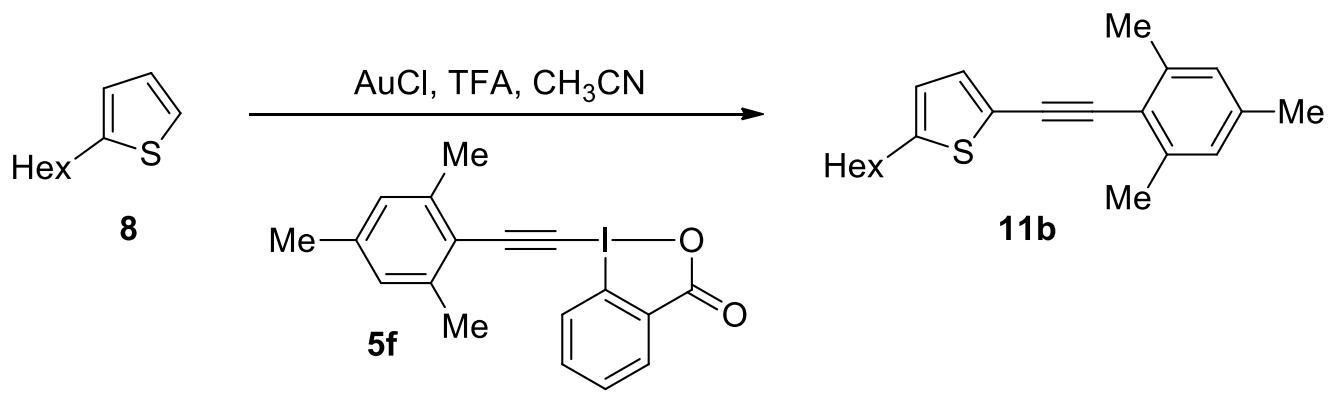

To a stirring solution of $\mathrm{AuCl}\left(4.6 \mathrm{mg}, 0.020 \mathrm{mmol}, 0.05\right.$ equiv) in $\mathrm{CH}_{3} \mathrm{CN}^{1}(2 \mathrm{~mL})$ was added 2hexylthiophene (8) $(72 \mu \mathrm{L}, 0.40 \mathrm{mmol}, 1$ equiv) under air. After $2 \mathrm{~min}$, TFA ( $36 \mu \mathrm{L}, 0.48 \mathrm{mmol}, 1.2$ equiv) and Mes-EBX (5f) (187 mg, $0.480 \mathrm{mmol}, 1.2$ equiv) were added. The reaction was sealed and stirred at RT for $14 \mathrm{~h}$. $\mathrm{Et}_{2} \mathrm{O}(10 \mathrm{~mL})$ was added, the organic layer was washed twice with $0.1 \mathrm{M} \mathrm{NaOH}(15 \mathrm{~mL})$. The aqueous layers were combined and extracted with $\mathrm{Et}_{2} \mathrm{O}(20 \mathrm{~mL})$. The organic layers were combined, washed with saturated $\mathrm{NaHCO}_{3}(20 \mathrm{~mL})$, brine $(20 \mathrm{~mL})$, dried with $\mathrm{MgSO}_{4}$ and concentrated under reduced pressure. The resulting oil was purified by flash chromatography (pentane) to afford $\mathbf{1 1 b}(33 \mathrm{mg}, 0.11$ mmol, $27 \%)$ as colorless oil. $\mathrm{R}_{\mathrm{f}}$ (pentane): 0.6. ${ }^{1} \mathrm{H} \mathrm{NMR}\left(400 \mathrm{MHz}, \mathrm{CDCl}_{3}\right) \delta 7.07(\mathrm{~d}, 1 \mathrm{H}, J=3.6 \mathrm{~Hz}$, ArH), 6.88 (s, $2 \mathrm{H}, \mathrm{ArH}), 6.68(\mathrm{~d}, 1 \mathrm{H}, J=3.6 \mathrm{~Hz}, \mathrm{ArH}), 2.80\left(\mathrm{t}, 2 \mathrm{H}, J=7.5 \mathrm{~Hz}, \mathrm{CH}_{2}\right), 2.44\left(\mathrm{~s}, 6 \mathrm{H}, \mathrm{CH}_{3}\right)$, 2.29 (s, $\left.3 \mathrm{H}, \mathrm{CH}_{3}\right), 1.68\left(\mathrm{~m}, 2 \mathrm{H}, \mathrm{CH}_{2}\right), 1.43-1.28\left(\mathrm{~m}, 6 \mathrm{H}, \mathrm{CH}_{2}\right), 0.90\left(\mathrm{~m}, 3 \mathrm{H}, \mathrm{CH}_{3}\right) .{ }^{13} \mathrm{C} \mathrm{NMR}(101 \mathrm{MHz}$, $\left.\mathrm{CDCl}_{3}\right) \delta 147.8,139.9,137.7,131.0,127.6,124.1,121.2,119.9,90.4,90.3,31.6,31.5,30.2,28.7,22.6$, 21.3, 21.0, 14.1. IR 2945 (s), 2907 (s), 2840 (m), $2175(\mathrm{w}), 1730$ (m), 1710 (m), 1603 (w), $1530(\mathrm{w}), 1481$ (m), 1454 (s), 1383 (m), 1244 (m), 1204 (m), 1113 (w), 1021 (m), 902 (m), 842 (w), 788 (m), 755 (m), 718 (s). HRMS (ESI) calcd for $\mathrm{C}_{21} \mathrm{H}_{27} \mathrm{~S}^{+}[\mathrm{M}+\mathrm{H}]^{+} 311.1828$; found 311.1844 .

\section{2-Hexyl-5-((4-nitrophenyl)ethynyl)thiophene (11c)}

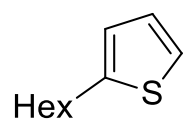

8

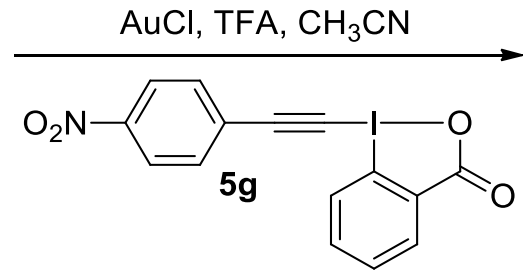

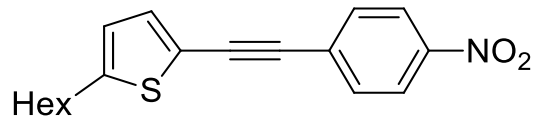

11c

To a stirring solution of $\mathrm{AuCl}\left(4.6 \mathrm{mg}, 0.020 \mathrm{mmol}, 0.05\right.$ equiv) in $\mathrm{CH}_{3} \mathrm{CN}^{1}(2 \mathrm{~mL})$ was added 2hexylthiophene (8) $(72 \mu \mathrm{L}, 0.40 \mathrm{mmol}, 1$ equiv) under air. After $2 \mathrm{~min}$, TFA (36 $\mu \mathrm{L}, 0.48 \mathrm{mmol}, 1.2$ equiv) and 4-NO ${ }_{2} \mathrm{Ph}-\mathrm{EBX}$ (5g) (167 $\mathrm{mg}, 0.480 \mathrm{mmol}, 1.2$ equiv) were added. The reaction was sealed and stirred at RT for $36 \mathrm{~h}$. $\mathrm{Et}_{2} \mathrm{O}(10 \mathrm{~mL})$ was added, the organic layer was washed twice with $0.1 \mathrm{M} \mathrm{NaOH}(15 \mathrm{~mL})$. The aqueous layers were combined and extracted with $\mathrm{Et}_{2} \mathrm{O}(20 \mathrm{~mL})$. The organic layers were combined, washed with saturated $\mathrm{NaHCO}_{3}(20 \mathrm{~mL})$, brine $(20 \mathrm{~mL})$, dried with $\mathrm{MgSO}_{4}$ and concentrated under reduced pressure. The resulting oil was purified by flash chromatography (pentane/EtOAc 99/1) to afford 11c (44 $\mathrm{mg}, 0.14 \mathrm{mmol}, 35 \%$ ) as colorless oil. $\mathrm{R}_{\mathrm{f}}$ (pentane/EtOAc 99/1): 0.3. ${ }^{1} \mathrm{H}$ NMR $\left(400 \mathrm{MHz}, \mathrm{CDCl}_{3}\right) \delta 8.20$ $(\mathrm{dm}, 2 \mathrm{H}, J=9.0 \mathrm{~Hz}, \mathrm{ArH}), 7.61(\mathrm{dm}, 2 \mathrm{H}, J=9.0 \mathrm{~Hz}, \mathrm{ArH}), 7.18(\mathrm{~d}, 1 \mathrm{H}, J=3.6 \mathrm{~Hz}, \mathrm{ArH}), 6.72(\mathrm{~d}, 1 \mathrm{H}, J$ $=3.6 \mathrm{~Hz}, \mathrm{ArH}), 2.81\left(\mathrm{t}, 2 \mathrm{H}, J=7.6 \mathrm{~Hz}, \mathrm{CH}_{2}\right), 1.67\left(\mathrm{~m}, 2 \mathrm{H}, \mathrm{CH}_{2}\right), 1.44-1.24\left(\mathrm{~m}, 6 \mathrm{H}, \mathrm{CH}_{2}\right), 0.89(\mathrm{~m}, 3 \mathrm{H}$, $\left.\mathrm{CH}_{3}\right) .{ }^{13} \mathrm{C} \mathrm{NMR}\left(101 \mathrm{MHz}, \mathrm{CDCl}_{3}\right) \delta 150.3,146.7,133.4,131.7,130.2,124.6,123.6,119.1,90.8,88.9$, 
31.5, 31.5, 30.3, 28.7, 22.5, 14.0. IR 3107 (w), 3078 (w), 2957 (w), 2930 (m), 2856 (w), 2196 (m), 1926 (w), 1594 (s), 1516 (s), 1459 (m), 1379 (w), 1340 (s), 1285 (w), 1212 (w), 1175 (w), 1107 (m), 1030 (w), 1030 (w), 911 (w), 854 (s), 804 (m), 749 (m), 687 (m). Not detectable by ESI. GC/MS: m/z: 313 $\left(\mathrm{MW}\left(\mathrm{C}_{18} \mathrm{H}_{19} \mathrm{NO}_{2} \mathrm{~S}\right): 313.1\right)$.

\section{2-(3,3-Dimethylbut-1-yn-1-yl)-5-hexylthiophene (11e)}
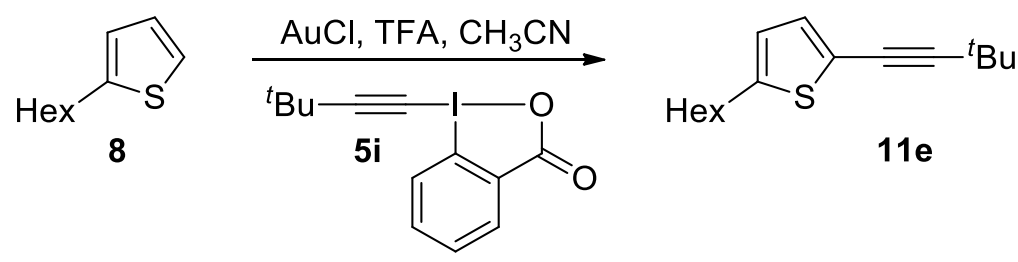

To a stirring solution of $\mathrm{AuCl}\left(4.6 \mathrm{mg}, 0.020 \mathrm{mmol}, 0.05\right.$ equiv) in $\mathrm{CH}_{3} \mathrm{CN} 1(2 \mathrm{~mL})$ was added 2hexylthiophene (8) $(72 \mu \mathrm{L}, 0.40 \mathrm{mmol}, 1$ equiv) under air. After $2 \mathrm{~min}$, TFA (36 $\mu \mathrm{L}, 0.48 \mathrm{mmol}, 1.2$ equiv) and ${ }^{t} \mathrm{Bu}-\mathrm{EBX}$ (5i) $(157 \mathrm{mg}, 0.480 \mathrm{mmol}, 1.2$ equiv) were added. The reaction was sealed and stirred at RT for $14 \mathrm{~h}$. $\mathrm{Et}_{2} \mathrm{O}(10 \mathrm{~mL})$ was added, the organic layer was washed twice with $0.1 \mathrm{M} \mathrm{NaOH}(15 \mathrm{~mL})$. The aqueous layers were combined and extracted with $\mathrm{Et}_{2} \mathrm{O}(20 \mathrm{~mL})$. The organic layers were combined, washed with saturated $\mathrm{NaHCO}_{3}(20 \mathrm{~mL})$, brine $(20 \mathrm{~mL})$, dried with $\mathrm{MgSO}_{4}$ and concentrated under reduced pressure. The resulting oil was purified by flash chromatography (pentane) to afford 11e (36 mg, $0.14 \mathrm{mmol}$, $36 \%$ ) as colorless oil. $\mathrm{R}_{\mathrm{f}}$ (pentane): 0.6. ${ }^{1} \mathrm{H} \mathrm{NMR}\left(400 \mathrm{MHz}, \mathrm{CDCl}_{3}\right) \delta 6.93(\mathrm{~d}, 1 \mathrm{H}, J=3.5 \mathrm{~Hz}, \mathrm{ArH}), 6.60$ $(\mathrm{d}, 1 \mathrm{H}, J=3.5 \mathrm{~Hz}, \mathrm{ArH}), 2.77\left(\mathrm{t}, 2 \mathrm{H}, J=7.6 \mathrm{~Hz}, \mathrm{CH}_{2}\right), 1.65\left(\mathrm{~m}, 2 \mathrm{H}, \mathrm{CH}_{2}\right), 1.40-1.24\left(\mathrm{~m}, 15 \mathrm{H}, \mathrm{CH}_{2}+\right.$ $\left.{ }^{t} \mathrm{Bu}\right), 0.91\left(\mathrm{~m}, 3 \mathrm{H}, \mathrm{CH}_{3}\right) .{ }^{13} \mathrm{C} \mathrm{NMR}\left(101 \mathrm{MHz}, \mathrm{CDCl}_{3}\right) \delta 146.7,130.8,123.7,121.4,101.4,72.5,31.6,31.5$, 30.9, 30.9, 30.1, 28.6, 22.6, 14.1. IR 2967 (s), 2921 (s), 2857 (s), 1736 (w), 1539 (w), 1458 (m), 1362 (m), $1274(\mathrm{~m}), 1224(\mathrm{~m}), 1204(\mathrm{~m}), 1156$ (w), 1033 (w), 910 (w), 884 (w), 799 (s), 735 (m). HRMS (ESI) calcd for $\mathrm{C}_{16} \mathrm{H}_{25} \mathrm{~S}^{+}[\mathrm{M}+\mathrm{H}]^{+} 249.1671$; found 249.1684.

\section{One pot deprotection-Sonogashira coupling}

\section{3-((4-Methoxyphenyl)ethynyl)-2-phenyl-1H-indole (46)}

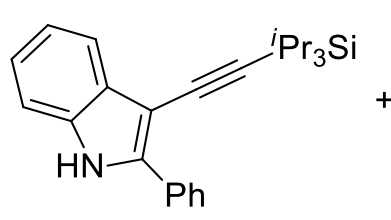

44<smiles>COc1ccc(I)cc1</smiles>

45

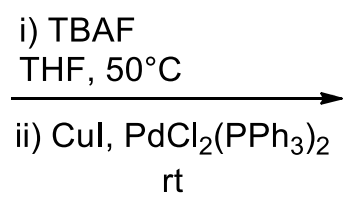

$\mathrm{rt}$

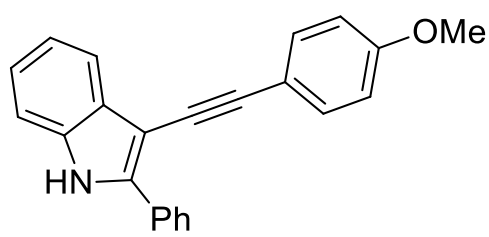

46

Following a reported procedure, ${ }^{11}$ TBAF $(1.0 \mathrm{M}$ in THF, $0.354 \mathrm{~mL}, 0.354 \mathrm{mmol}, 2.0$ equiv) was added dropwise to a solution of 2-phenyl-3-((triisopropylsilyl)ethynyl)- $1 H$-indole (44) ${ }^{12}$ (66 mg, $0.18 \mathrm{mmol}, 1.0$ equiv) in THF ( $1 \mathrm{~mL})$ under nitrogen and the mixture was stirred at $50{ }^{\circ} \mathrm{C}$ for $1 \mathrm{~h}$. It was then allowed to cool down to RT and $p$-iodoanisole (45) (51 $\mathrm{mg}, 0.21 \mathrm{mmol}, 1.2$ equiv) was added, followed by $\mathrm{PdCl}_{2}\left(\mathrm{PPh}_{3}\right)_{2}$ (10 mg, $0.014 \mathrm{mmol}, 0.08$ equiv) and $\mathrm{CuI}$ (41 mg, $0.21 \mathrm{mmol}, 1.2$ equiv). After stirring at $\mathrm{RT}$ overnight, $\mathrm{SiO}_{2}$ was added and the solvent was removed in vacuo. Column chromatography purification of the crude product pre-adsorbed on silica gel (Pentane/Et ${ }_{2} \mathrm{O}$ 9/1) afforded $\mathbf{4 6}$ (46 mg, $0.14 \mathrm{mmol}, 81 \%$ ) as a

\footnotetext{
${ }^{11}$ Sun, J. W.; Conley, M. P.; Zhang, L. M.; Kozmin, S. A. J. Am. Chem. Soc. 2006, 128, 9705.

${ }^{12}$ Brand, J. P.; Chevalley, C.; Waser, J. Beilstein J. Org. Chem. 2011, 7, 565.
} 
yellow solid. $\mathrm{R}_{\mathrm{f}}$ (Pentane/Et $2 \mathrm{O}$ 9/1): 0.15. ${ }^{1} \mathrm{H}$ NMR (400 MHz, $\mathrm{CDCl}_{3}$ ) $\delta 8.44$ (br s, $1 \mathrm{H}, \mathrm{NH}$ ), 8.04 (dd, $2 \mathrm{H}$, $J=8.5,1.3 \mathrm{~Hz}, \operatorname{ArH}), 7.83(\mathrm{~d}, 1 \mathrm{H}, J=6.7 \mathrm{~Hz}, \operatorname{ArH}), 7.49(\mathrm{~m}, 4 \mathrm{H}, \mathrm{ArH}), 7.37$ (m, $2 \mathrm{H}, \operatorname{ArH}), 7.23(\mathrm{~m}, 2 \mathrm{H}$, $\mathrm{ArH}), 6.90(\mathrm{~m}, 2 \mathrm{H}, \mathrm{ArH}), 3.83$ (s, $3 \mathrm{H}, \mathrm{Me}) .{ }^{13} \mathrm{C} \mathrm{NMR}\left(101 \mathrm{MHz}, \mathrm{CDCl}_{3}\right) \delta 159.1,139.0,135.3,132.7$, 131.6, 130.3, 128.9, 128.2, 126.5, 123.4, 120.8, 120.1, 116.5, 114.0, 111.0, 96.2, 93.3, 82.5, 55.3. IR 3412 (w), $3057(\mathrm{w}), 2960(\mathrm{w}), 2934(\mathrm{w}), 2836(\mathrm{w}), 2205(\mathrm{w}), 1889(\mathrm{w}), 1604(\mathrm{~m}), 1569(\mathrm{w}), 1511(\mathrm{~m}), 1491(\mathrm{~m})$, $1451(\mathrm{~m}), 1432(\mathrm{~m}), 1374(\mathrm{w}), 1326(\mathrm{w}), 1288(\mathrm{~m}), 1246$ (s), $1174(\mathrm{~m}), 1143(\mathrm{w}), 1107$ (w), $1029(\mathrm{~m}), 908$ $(\mathrm{w}), 823(\mathrm{~m}), 743(\mathrm{~s}), 693(\mathrm{~s}), 623(\mathrm{w})$. HRMS (ESI) calcd for $\mathrm{C}_{23} \mathrm{H}_{18} \mathrm{NO}^{+}[\mathrm{M}+\mathrm{H}]^{+} 324.1383$; found 324.1390 .

\section{2-Hexyl-5-((4-methoxyphenyl)ethynyl)thiophene (47)}
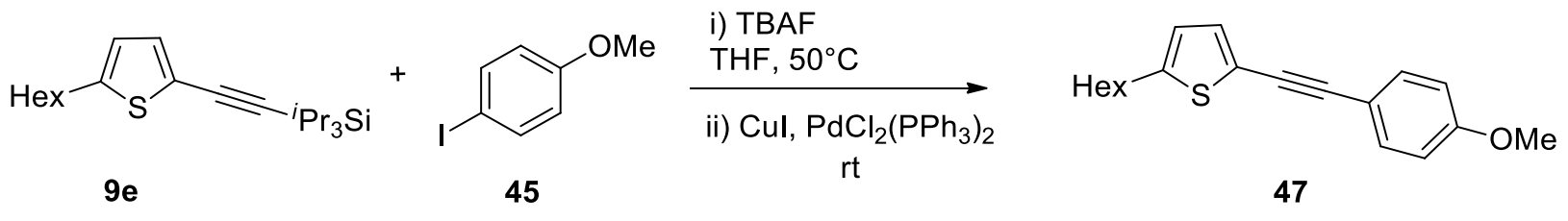

Following a reported procedure, TBAF $(1.0 \mathrm{M}$ in THF, $0.856 \mathrm{~mL}, 0.856 \mathrm{mmol} 2.0$ equiv) was added dropwise to a solution of ((5-hexylthiophen-2-yl)ethynyl)triisopropylsilane (9e) (149 mg, $0.428 \mathrm{mmol}, 1.0$ equiv) in THF $(2.2 \mathrm{~mL})$ under nitrogen and the mixture was stirred at $50^{\circ} \mathrm{C}$ for $1 \mathrm{~h}$. It was then allowed to cool down to RT and $p$-iodoanisole (45) (124 mg, $0.514 \mathrm{mmol}, 1.2$ equiv) was added followed by $\mathrm{PdCl}_{2}\left(\mathrm{PPh}_{3}\right)_{2}$ (24 mg, $0.034 \mathrm{mmol}, 0.08$ equiv) and $\mathrm{CuI}$ (98 mg, $0.51 \mathrm{mmol}, 1.2$ equiv). After stirring at $\mathrm{RT}$ for $3 \mathrm{~h}, \mathrm{SiO}_{2}$ was added and the solvent was removed in vacuo. Column chromatography purification of the crude product pre-adsorbed on silica gel (Pentane/ $\mathrm{CH}_{2} \mathrm{Cl}_{2}$ 99/1) afforded 47 (81 $\mathrm{mg}, 0.27 \mathrm{mmol}, 63 \%$ ) as a yellow oil. $\mathrm{R}_{\mathrm{f}}$ (Pentane/ $\mathrm{CH}_{2} \mathrm{Cl}_{2}$ 99/1): 0.15. ${ }^{1} \mathrm{H} \mathrm{NMR}\left(400 \mathrm{MHz}, \mathrm{CDCl}_{3}\right) \delta 7.45(\mathrm{~d}, 2 \mathrm{H}, J=8.9 \mathrm{~Hz}, \mathrm{ArH})$, $7.08(\mathrm{~d}, 1 \mathrm{H}, J=3.6 \mathrm{~Hz}, \operatorname{ArH}), 6.88(\mathrm{~d}, 2 \mathrm{H}, J=8.9 \mathrm{~Hz}, \mathrm{ArH}), 6.67(\mathrm{~d}, 1 \mathrm{H}, J=3.6 \mathrm{~Hz}, \mathrm{ArH}), 3.82(\mathrm{~s}, 3 \mathrm{H}$, $\mathrm{Me}), 2.80\left(\mathrm{t}, 2 \mathrm{H}, J=7.4 \mathrm{~Hz}, \mathrm{CH}_{2}\right), 1.68\left(\mathrm{~m}, 2 \mathrm{H}, \mathrm{CH}_{2}\right), 1.41-1.30\left(\mathrm{~m}, 6 \mathrm{H}, \mathrm{CH}_{2}\right), 0.91\left(\mathrm{~m}, 3 \mathrm{H}, \mathrm{CH}_{3}\right) .{ }^{13} \mathrm{C}$ NMR (101 MHz, $\left.\mathrm{CDCl}_{3}\right) \delta 159.5,147.8,132.8,131.4,124.1,120.8,115.2,113.9,92.2,81.7,55.2,31.5$, 31.5, 30.2, 28.7, 22.5, 14.0. IR $3072(\mathrm{w}), 3002(\mathrm{w}), 2955(\mathrm{~m}), 2850(\mathrm{~m}), 2541(\mathrm{w}), 2203(\mathrm{w}), 2058(\mathrm{w}), 1887$ (w), 1757 (w), 1606 (s), 1569 (w), 1539 (m), 1515 (m), 1510 (s), 1464 (s), 1440 (m), 1378 (w), 1291 (s), 1173 (s), 1109 (m), 1034 (s), 831 (s), 801 (s), 732 (w). HRMS (ESI) calcd for $\mathrm{C}_{19} \mathrm{H}_{23} \mathrm{OS}^{+}[\mathrm{M}+\mathrm{H}]^{+}$299.1464; found 299.1463.

\section{Triisopropylsilyl benziodoxol(on)e analogues}

\section{5-Methyl-2-iodosylbenzoic acid (13a)}<smiles>Cc1ccc(I)c(C(=O)O)c1</smiles>
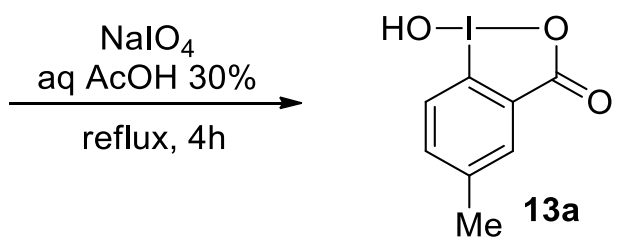

Following the reported procedure, ${ }^{13} \mathrm{NaIO}_{4}(1.25 \mathrm{~g}, 5.84 \mathrm{mmol}, 1.05$ equiv) and 2-iodo-5-methylbenzoic acid (48) (1.46 g, $5.56 \mathrm{mmol}, 1.00$ equiv) were suspended in $30 \%$ (v:v) aq. AcOH (15 mL). The mixture was vigorously stirred and refluxed for $4 \mathrm{~h}$. The reaction mixture was then diluted with cold water $(40 \mathrm{~mL})$ and allowed to cool to RT, protecting it from light. The crude product was collected by filtration, washed on the filter with ice water $(3 \times 4 \mathrm{~mL})$ and acetone $(3 \times 4 \mathrm{~mL})$, and air-dried in the dark to give the pure product

${ }^{13}$ L. Kraszkiewicz, L. Skulski, Arkivoc. 2003, 6, 120. 
13a (1.39 g, $5.00 \mathrm{mmol}, 90 \%)$ as a colorless solid. ${ }^{1} \mathrm{H}$ NMR (400 MHz, DMSO) $\delta 7.84$ (s, $\left.1 \mathrm{H}, \mathrm{ArH}\right), 7.78$ (m, $1 \mathrm{H}, \mathrm{ArH}), 7.69$ (m, $1 \mathrm{H}, \mathrm{ArH}), 2.47$ (s, $\left.3 \mathrm{H}, \mathrm{CH}_{3}\right)$.

5-Methyl-1-[(triisopropylsilyl)ethynyl]-1,2-benziodoxol-3(1H)-one (15a)

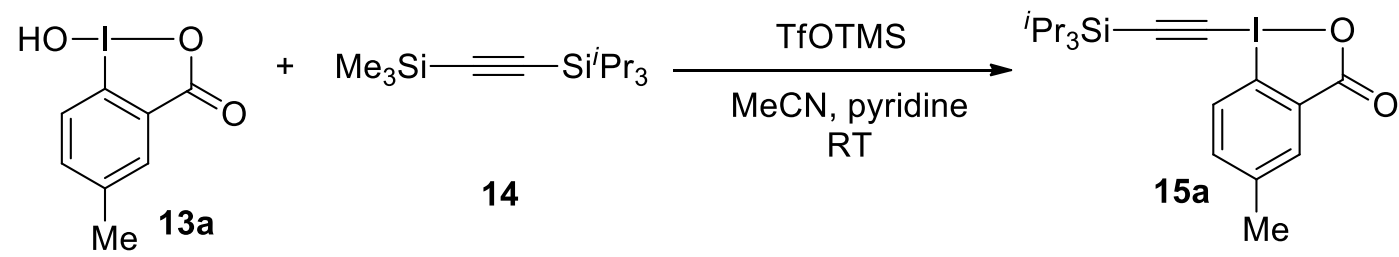

Trimethylsilyltriflate $(400 \mu \mathrm{L}, 2.20 \mathrm{mmol}, 1.1$ equiv) was added dropwise to a stirred solution of 13a (556 mg, $2.00 \mathrm{mmol}, 1.0$ equiv) in acetonitrile $(10 \mathrm{~mL})$. After $20 \mathrm{~min}$, (trimethylsilyl)(triisopropylsilyl)acetylene (14) $(560 \mathrm{mg}, 2.20 \mathrm{mmol}, 1.1$ equiv) was then added dropwise, followed, after $20 \mathrm{~min}$, by the addition of pyridine ( $180 \mu \mathrm{L}, 2.20 \mathrm{mmol}, 1.1$ equiv). The mixture was stirred $20 \mathrm{~min}$. The solvent was then removed under reduced pressure and the yellow crude oil was dissolved in dichloromethane $(20 \mathrm{~mL})$. The organic layer was washed with $1 \mathrm{M} \mathrm{HCl}(20 \mathrm{~mL})$ and the aqueous layer was extracted with $\mathrm{CH}_{2} \mathrm{Cl}_{2}(20$ $\mathrm{mL})$. The organic layers were combined, washed with a saturated solution of $\mathrm{NaHCO}_{3}(40 \mathrm{~mL})$, dried over $\mathrm{MgSO}_{4}$, filtered and the solvent was evaporated under reduced pressure. Recrystallization from acetonitrile (ca $25 \mathrm{~mL}$ ) and wash with hexanes afforded 15a (559 mg, $1.26 \mathrm{mmol}, 63 \%$ ) as colorless cristals. Mp (Dec.) $192-197^{\circ} \mathrm{C} .{ }^{1} \mathrm{H}$ NMR $\left(400 \mathrm{MHz}, \mathrm{CDCl}_{3}\right)(c a 0.11 \mathrm{mmol} / \mathrm{mL}) \delta 8.23(\mathrm{~d}, 1 \mathrm{H}, J=1.5 \mathrm{~Hz}, \mathrm{ArH}), 8.12(\mathrm{~d}, 1$ $\mathrm{H}, J=8.5 \mathrm{~Hz}, \mathrm{ArH}), 7.57(\mathrm{dd}, 1 \mathrm{H}, J=8.5,1.8 \mathrm{~Hz}, \mathrm{ArH}), 2.51(\mathrm{~s}, 3 \mathrm{H}, \mathrm{Me}), 1.16$ (m, $21 \mathrm{H}, \mathrm{TIPS}) .{ }^{13} \mathrm{C}$ NMR $\left(101 \mathrm{MHz} \mathrm{CDCl}_{3}\right) \delta 166.6,142.5,135.6,133.0,131.2,125.8,113.8,111.8,64.6,20.7,18.5,11.2$. The characterization data corresponded to the reported values. ${ }^{14}$ The crystal structure of $\mathbf{5 d}$ has been deposited at the Cambridge Crystallographic Data Centre and allocated the deposition number: CCDC 863347.

\section{5-Fluoro-2-iodosylbenzoic acid (13b)}

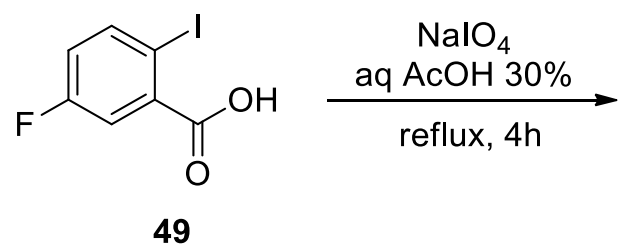<smiles>O=C1OI(O)c2ccc(F)cc21</smiles>

Following the reported procedure, $\mathrm{NaIO}_{4}(656 \mathrm{mg}, 3.07 \mathrm{mmol}, 1.05$ equiv) and 2-iodo-4-fluorobenzoic acid (49) (778 mg, $2.92 \mathrm{mmol}, 1.00$ equiv) were suspended in $30 \%$ (v:v) aq. AcOH (7 mL). The mixture was vigorously stirred and refluxed for $4 \mathrm{~h}$. The reaction mixture was then diluted with cold water $(20 \mathrm{~mL})$ and allowed to cool to RT, protecting it from light. The crude product was collected by filtration, washed on the filter with ice water $(3 \times 4 \mathrm{~mL})$ and acetone $(3 \times 4 \mathrm{~mL})$, and air-dried in the dark to give the pure product 13b (738 mg, $2.62 \mathrm{mmol}, 90 \%$ ) as a colorless solid. ${ }^{1} \mathrm{H}$ NMR (400 MHz, DMSO) $\delta$ 7.88-7.79 (m, $3 \mathrm{H}, \mathrm{ArH}$ $+\mathrm{OH}), 7.75(\mathrm{~m}, 1 \mathrm{H}, \mathrm{ArH})$.

\section{5-Fluoro-1-[(Triisopropylsilyl)ethynyl]-1,2-benziodoxol-3(1H)-one (15b)}

\footnotetext{
${ }^{14}$ Ohta, Y.; Tokimizu, Y.; Oishi, S.; Fujii, N.; Ohno, H. Org. Lett. 2010, 12, 3963.
} 

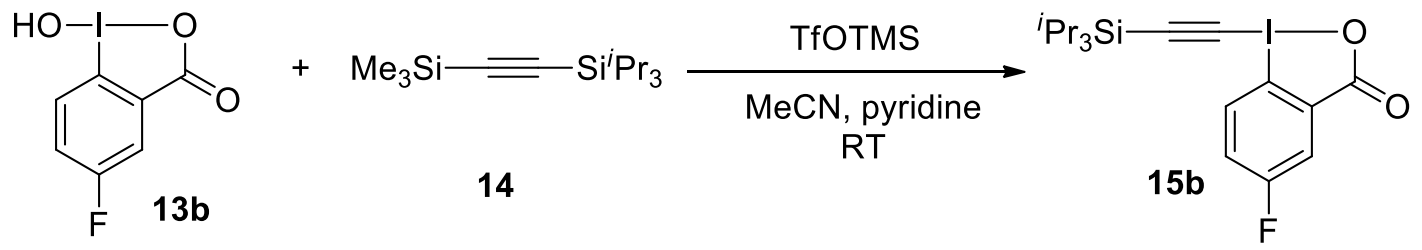

Trimethylsilyltriflate ( $247 \mu \mathrm{L}, 1.36 \mathrm{mmol}, 1.1$ equiv, freshly distilled) was added dropwise to a stirred solution of $\mathbf{1 3 b}$ ( $350 \mathrm{mg}, 1.24 \mathrm{mmol}, 1.0$ equiv) in acetonitrile (5 mL). (Trimethylsilyl)(triisopropylsilyl)acetylene (14) (347 mg, $1.36 \mathrm{mmol}, 1.1$ equiv) was then added dropwise, followed, after $15 \mathrm{~min}$, by the addition of pyridine ( $110 \mu \mathrm{L}, 1.36 \mathrm{mmol}, 1.1$ equiv). The mixture was stirred $10 \mathrm{~min}$. The solvent was then removed under reduced pressure and the yellow crude oil was dissolved in dichloromethane $(50 \mathrm{~mL})$. The organic layer was washed with $1 \mathrm{M} \mathrm{HCl}(50 \mathrm{~mL})$ and the aqueous layer was extracted with $\mathrm{CH}_{2} \mathrm{Cl}_{2}(50$ $\mathrm{mL})$. The organic layers were combined, washed with a saturated solution of $\mathrm{NaHCO}_{3}(2 \times 50 \mathrm{~mL})$, dried over $\mathrm{MgSO}_{4}$, filtered and the solvent was evaporated under reduced pressure. Recrystallization from acetonitrile ( ca $5 \mathrm{~mL}$ ) afforded $15 \mathrm{~b}(381 \mathrm{mg}, 0.854 \mathrm{mmol}, 69 \%)$ as a colorless solid. Mp (Dec.) 185 $189^{\circ} \mathrm{C} .{ }^{1} \mathrm{H}$ NMR $\left(400 \mathrm{MHz}, \mathrm{CDCl}_{3}\right)(c a 0.04 \mathrm{mmol} / \mathrm{mL}) \delta 8.22(\mathrm{dd}, 1 \mathrm{H}, J=9.0,4.2 \mathrm{~Hz}, \mathrm{ArH}), 8.10(\mathrm{dd}, 1$ $\mathrm{H}, J=7.9,2.9 \mathrm{~Hz}, \mathrm{ArH}), 7.48(\mathrm{~m}, 1 \mathrm{H}, \mathrm{ArH}), 1.16$ (m, $21 \mathrm{H}, \mathrm{TIPS}) .{ }^{13} \mathrm{C} \mathrm{NMR}\left(101 \mathrm{MHz}, \mathrm{CDCl}_{3}\right) \delta 165.6$ $(\mathrm{d}, J=254 \mathrm{~Hz}), 165.2(\mathrm{~d}, J=7 \mathrm{~Hz}), 134.2(\mathrm{~d}, J=7 \mathrm{~Hz}), 127.8(\mathrm{~d}, J=8 \mathrm{~Hz}), 122.2(\mathrm{~d}, J=24 \mathrm{~Hz}), 119.4(\mathrm{~d}$, $J=24 \mathrm{~Hz}), 115.0(\mathrm{~s}), 108.0(\mathrm{~d}, J=1 \mathrm{~Hz}), 64.0(\mathrm{~s}), 18.5(\mathrm{~s}), 11.2(\mathrm{~s})$. The characterization data corresponded to the reported values. ${ }^{14}$

2-Iodosyl-5-nitrobenzoic acid (13c) and 2-iodosyl-3-nitrobenzoic acid (50)

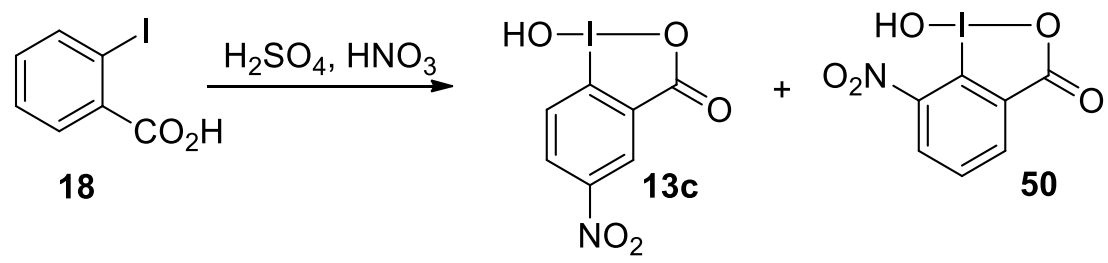

Following a reported procedure, ${ }^{15}$ fuming nitric acid $(3.3 \mathrm{~mL})$ was acid to 2-iodobenzoic acid (18) (5.0 g, 20 mmol, 1 equiv) in concentrated $\mathrm{H}_{2} \mathrm{SO}_{4}(6.7 \mathrm{~mL})$. The reaction was equipped with a cooler and a nitrous vapor trap and was heated at $100^{\circ} \mathrm{C}$ for $1 \mathrm{~h}$. The reaction mixture was then poured in ice-water and filtered. The resulting solid was refluxed in water $(50 \mathrm{~mL})$ and filtered. A second crop of precipitate was filtered from the mother liquors. Both solids were combined, washed with acetone $(10 \mathrm{~mL})$ and dried under vacuum to afford $13 \mathrm{c}(2.19 \mathrm{~g}, 7.10 \mathrm{mmol}, 36 \%)$. The mother liquors were reduced to one third and then kept at $4{ }^{\circ} \mathrm{C}$, the resulting precipitate was filtered, washed with acetone $(10 \mathrm{~mL})$ and dried under vacuum to afford $\mathbf{5 0}$ (630 mg, 2.04 mmol, $10 \%$ ). 13c: ${ }^{1} \mathrm{H}$ NMR (400 MHz, DMSO) $\delta 8.73$ (dd, $1 \mathrm{H}, J=8.8,2.6 \mathrm{~Hz}, \operatorname{ArH}$ ), 8.58 $(\mathrm{d}, 1 \mathrm{H}, \quad J=2.4 \mathrm{~Hz}, \mathrm{ArH}), 8.54($ br s, $1 \mathrm{H}, \mathrm{OH}), 8.11(\mathrm{~d}, 1 \mathrm{H}, J=8.8 \mathrm{~Hz}, \mathrm{ArH}) .50:{ }^{1} \mathrm{H} \mathrm{NMR}(400 \mathrm{MHz}$, DMSO) $\delta 7.92(\mathrm{dd}, 1 \mathrm{H}, J=7.9,1.5 \mathrm{~Hz}), 7.79(\mathrm{~m}, 1 \mathrm{H}), 7.67(\mathrm{~m}, 1 \mathrm{H})$.

\section{5-Nitro-1-[(Triisopropylsilyl)ethynyl]-1,2-benziodoxol-3(1H)-one (15c)}

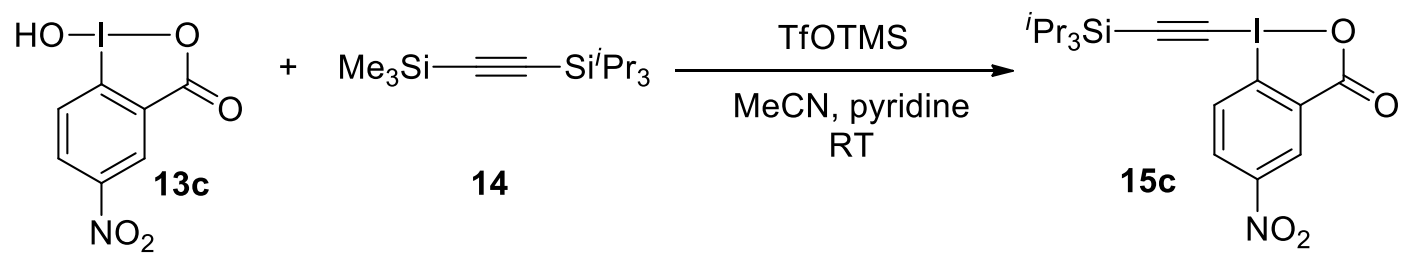

\footnotetext{
${ }^{15}$ Morrison, G. F.; Hooz, J. J. Org. Chem. 1970, 35, 1196
} 
Trimethylsilyltriflate $\left(646 \mu \mathrm{L}, 3.56 \mathrm{mmol}, 1.1\right.$ equiv, freshly distilled over $\left.\mathrm{CaH}_{2}\right)$ was added dropwise to a stirred solution of 2-iodosylbenzoic acid $13 \mathrm{c}\left(1.00 \mathrm{~g}, 3.23 \mathrm{mmol}, 1.0\right.$ equiv) in acetonitrile $(15 \mathrm{~mL})$ at $0^{\circ} \mathrm{C}$. After $15 \mathrm{~min}$ at RT, (trimethylsilyl)(triiso-propylsilyl)acetylene (14) (906 mg, $3.56 \mathrm{mmol}, 1.1$ equiv) was then added dropwise, followed, after $30 \mathrm{~min}$, by the addition of pyridine ( $290 \mu \mathrm{L}, 3.56 \mathrm{mmol}, 1.1$ equiv). The mixture was stirred $20 \mathrm{~min}$. The solvent was then removed under reduced pressure and the yellow crude oil was dissolved in dichloromethane $(25 \mathrm{~mL})$. The organic layer was washed with $1 \mathrm{M} \mathrm{HCl}(25 \mathrm{~mL})$ and the aqueous layer was extracted with $\mathrm{CH}_{2} \mathrm{Cl}_{2}(25 \mathrm{~mL})$. The organic layers were combined, washed with a saturated solution of $\mathrm{NaHCO}_{3}(20 \mathrm{~mL})$, dried over $\mathrm{MgSO}_{4}$, filtered and the solvent was evaporated under reduced pressure. Recrystallization from acetonitrile ( $\mathrm{ca} 20 \mathrm{~mL})$ afforded $15 \mathrm{c}(960 \mathrm{mg}, 2.02 \mathrm{mmol}, 63 \%)$ as a colorless solid. $\mathrm{Mp}$ (Dec.) $198-206^{\circ} \mathrm{C} .{ }^{1} \mathrm{H} \mathrm{NMR}\left(400 \mathrm{MHz}, \mathrm{CDCl}_{3}\right)(\mathrm{ca} 0.13 \mathrm{mmol} / \mathrm{mL}) \delta 9.20(\mathrm{~d}, 1 \mathrm{H}, J$ $=2.6 \mathrm{~Hz}, \mathrm{ArH}), 8.60(\mathrm{ddd}, 1 \mathrm{H}, J=9.0,2.5,0.4 \mathrm{~Hz}, \mathrm{ArH}), 8.53$ (d, $1 \mathrm{H}, J=8.9 \mathrm{~Hz}, \mathrm{ArH}), 1.30-1.14$ (m, 21 $\mathrm{H}$, TIPS). ${ }^{13} \mathrm{C}$ NMR $\left(101 \mathrm{MHz}, \mathrm{CDCl}_{3}\right) \delta 166.4,150.7,134.4,129.0,128.2,126.5,122.7,115.2,63.1,18.5$, 11.3. The characterization data for compound $21 \mathrm{c}$ corresponded to the reported values. ${ }^{14}$

\section{4,5-Dimethoxy-2-iodosylbenzoic acid (13d)}
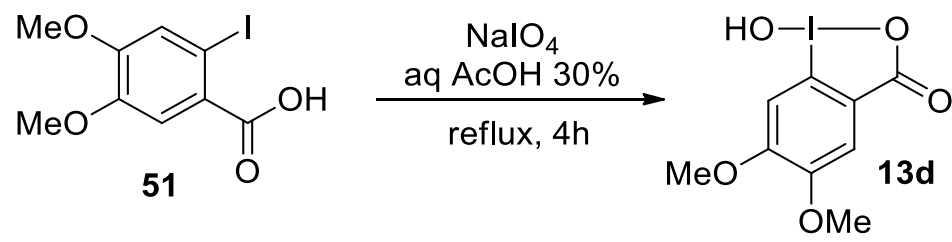

Following the reported procedure, ${ }^{13} \mathrm{NaIO}_{4}(1.25 \mathrm{~g}, 5.84 \mathrm{mmol}, 1.05$ equiv) and 2-iodo-4,5dimethoxybenzoic acid (51) (1.71 g, $5.56 \mathrm{mmol}, 1.00$ equiv) were suspended in $30 \%$ (v:v) aq. AcOH (15 $\mathrm{mL})$. The mixture was vigorously stirred and refluxed for $4 \mathrm{~h}$. The reaction mixture was then diluted with cold water $(40 \mathrm{~mL})$ and allowed to cool to RT, protecting it from light. The crude product was collected by filtration, washed on the filter with ice water $(3 \times 4 \mathrm{~mL})$ and acetone $(3 \times 4 \mathrm{~mL})$, and air-dried in the dark to give the pure product $\mathbf{1 3 d}(1.64 \mathrm{~g}, 5.06 \mathrm{mmol}, 91 \%)$ as a colorless solid. ${ }^{1} \mathrm{H}$ NMR (400 MHz, DMSO) $\delta 7.45$ (s, $1 \mathrm{H}, \mathrm{ArH}), 7.23$ (s, $1 \mathrm{H}, \mathrm{ArH}), 3.88(\mathrm{~d}, 6 \mathrm{H}, J=0.9 \mathrm{~Hz}, \mathrm{Me})$.

\section{4,5-Dimethoxy-1-[(triisopropylsilyl)ethynyl]-1,2-benziodoxol-3(1H)-one (15d)}

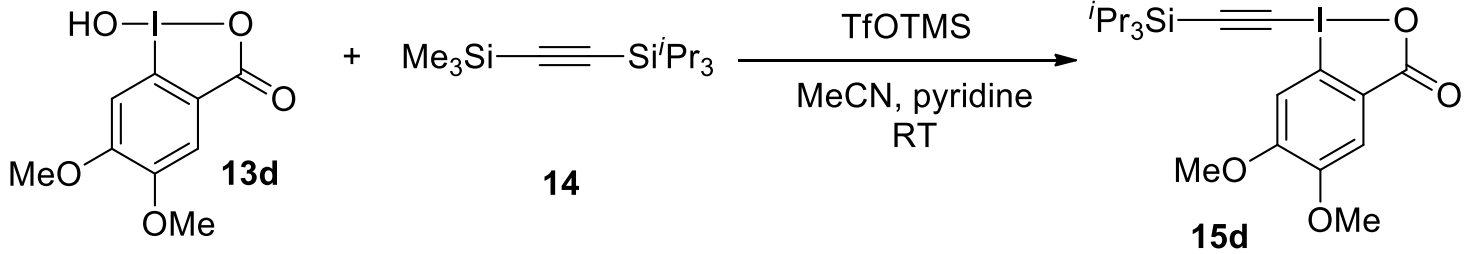

Trimethylsilyltriflate ( $400 \mu \mathrm{L}, 2.20 \mathrm{mmol}, 1.1$ equiv, freshly distilled) was added dropwise to a stirred solution of 13d (648 mg, $2.00 \mathrm{mmol}, 1.0$ equiv) in acetonitrile $(10 \mathrm{~mL})$. After $2 \mathrm{~min}$, (trimethylsilyl)(triisopropylsilyl)acetylene (14) (560 mg, $2.20 \mathrm{mmol}, 1.1$ equiv) was added dropwise, followed, after $20 \mathrm{~min}$, by the addition of pyridine ( $180 \mu \mathrm{L}, 2.20 \mathrm{mmol}, 1.1$ equiv). The mixture was stirred $20 \mathrm{~min}$. The solvent was then removed under reduced pressure and the yellow crude oil was dissolved in dichloromethane $(20 \mathrm{~mL})$. The organic layer was washed with $1 \mathrm{M} \mathrm{HCl}(20 \mathrm{~mL})$ and the aqueous layer was extracted with $\mathrm{CH}_{2} \mathrm{Cl}_{2}(20$ $\mathrm{mL})$. The organic layers were combined, washed with a saturated solution of $\mathrm{NaHCO}_{3}(40 \mathrm{~mL})$, dried over $\mathrm{MgSO}_{4}$, filtered and the solvent was evaporated under reduced pressure. Recrystallization from acetonitrile (ca $8 \mathrm{~mL}$ ) and wash with hexanes afforded 15d (575 mg, $1.18 \mathrm{mmol}, 59 \%)$ as colorless cristals. Mp (Dec.) $180-183^{\circ} \mathrm{C} .{ }^{1} \mathrm{H}$ NMR $\left(400 \mathrm{MHz}, \mathrm{CDCl}_{3}\right)(c a 0.09 \mathrm{mmol} / \mathrm{mL}) \delta 7.83(\mathrm{~s}, 1 \mathrm{H}, \mathrm{ArH}), 7.61(\mathrm{~s}, 1 \mathrm{H}, \mathrm{ArH}), 3.99$ (s, $3 \mathrm{H}, \mathrm{OMe}$ ), 3.97 (s, $3 \mathrm{H}, \mathrm{OMe}), 1.14$ (m, $21 \mathrm{H}, \mathrm{TIPS}) .{ }^{13} \mathrm{C} \mathrm{NMR}\left(101 \mathrm{MHz}, \mathrm{CDCl}_{3}\right) \delta 166.7,154.9$, 
152.2, 124.5, 113.8, 113.2, 107.8, 104.7, 66.0, 56.7, 56.5, 18.5, 11.2. IR $2945(\mathrm{w}), 1616(\mathrm{~m}), 1569(\mathrm{w}), 1497$ (m), $1464(\mathrm{w}), 1396(\mathrm{~m}), 1317(\mathrm{w}), 1269(\mathrm{~m}), 1215(\mathrm{~m}), 1181(\mathrm{w}), 1129(\mathrm{w}), 1026(\mathrm{w}), 921(\mathrm{w}), 884(\mathrm{w})$, 778 (w), 734 (m), 708 (m), 639 (s). HRMS(ESI) calcd for $\mathrm{C}_{20} \mathrm{H}_{30} \mathrm{O}_{4} \mathrm{ISi}^{+}(\mathrm{M}+\mathrm{H})$ 489.0958, found 489.0950. The crystal structure of $\mathbf{5 d}$ has been deposited at the Cambridge Crystallographic Data Centre and allocated the deposition number: CCDC 863348.

\section{3-Methyl-2-iodosylbenzoic acid (13e)}

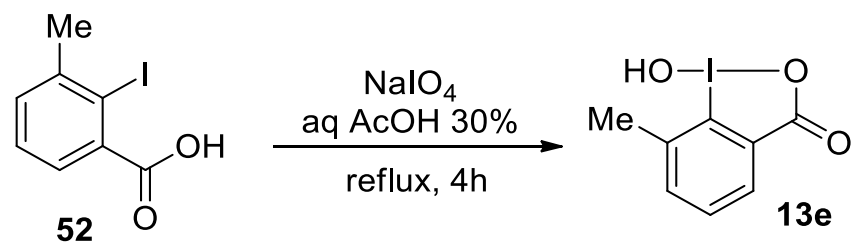

Following the reported procedure, ${ }^{13} \mathrm{NaIO}_{4}(1.25 \mathrm{~g}, 5.84 \mathrm{mmol}, 1.05$ equiv) and 2-iodo-3-methylbenzoic acid (52) (1.46 g, $5.56 \mathrm{mmol}, 1.00$ equiv) were suspended in 30\% (v:v) aq. AcOH (15 mL). The mixture was vigorously stirred and refluxed for $4 \mathrm{~h}$. The reaction mixture was then diluted with cold water $(40 \mathrm{~mL})$ and allowed to cool to RT, protecting it from light. The crude product was collected by filtration, washed on the filter with ice water $(3 \times 4 \mathrm{~mL})$ and acetone $(3 \times 4 \mathrm{~mL})$, and air-dried in the dark to give the pure product 13e (1.24 g, $4.46 \mathrm{mmol}, 80 \%)$ as a colorless solid. ${ }^{1} \mathrm{H}$ NMR (400 MHz, DMSO) $\delta 8.30$ (br s, $\left.1 \mathrm{H}, \mathrm{OH}\right), 7.85$ (m, 1 H, ArH), 7.57 (m, 2 H, ArH), 2.64 (s, 3 H, ArH).

\section{3-Methyl-1-[(triisopropylsilyl)ethynyl]-1,2-benziodoxol-3(1H)-one (15e)}

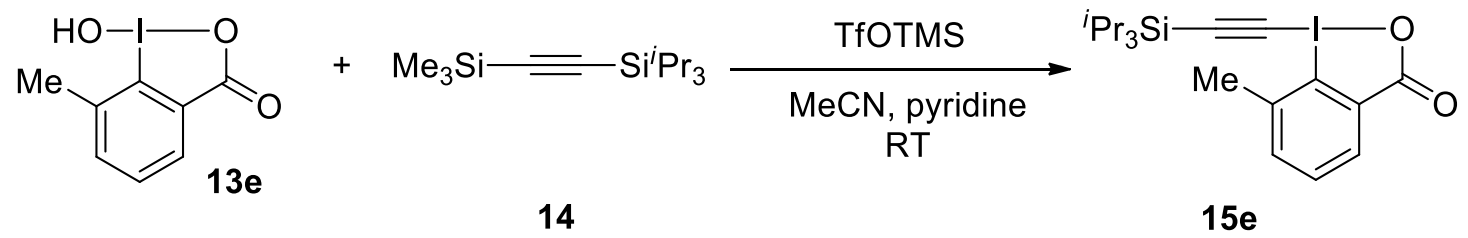

Trimethylsilyltriflate $(2.10 \mathrm{~mL}, 11.6 \mathrm{mmol}, 1.1$ equiv) was added dropwise to a stirred solution of $\mathbf{1 3 e}$ $(2.93 \mathrm{~g}, 10.5 \mathrm{mmol}, 1.0$ equiv) in acetonitrile $(45 \mathrm{~mL})$. After $20 \mathrm{~min}$, (trimethylsilyl)(triisopropylsilyl)acetylene (14) $(2.94 \mathrm{~g}, 11.6 \mathrm{mmol}, 1.1 \mathrm{equiv})$ was then added dropwise, followed, after $30 \mathrm{~min}$, by the addition of pyridine ( $934 \mu \mathrm{L}, 11.6 \mathrm{mmol}, 1.1$ equiv). The mixture was stirred $20 \mathrm{~min}$. The solvent was then removed under reduced pressure and the yellow crude oil was dissolved in dichloromethane $(30 \mathrm{~mL})$. The organic layer was washed with $1 \mathrm{M} \mathrm{HCl}(20 \mathrm{~mL})$ and the aqueous layer was extracted with $\mathrm{CH}_{2} \mathrm{Cl}_{2}(30$ $\mathrm{mL})$. The organic layers were combined, washed with a saturated solution of $\mathrm{NaHCO}_{3}(40 \mathrm{~mL})$, dried over $\mathrm{MgSO}_{4}$, filtered and the solvent was evaporated under reduced pressure. Recrystallization from acetonitrile ( ca $10 \mathrm{~mL}$ ) and wash with pentane afforded $15 \mathrm{e}(2.79 \mathrm{~g}, 6.31 \mathrm{mmol}, 60 \%)$ as colorless cristals. Mp (Dec.) $138-145^{\circ} \mathrm{C} .{ }^{1} \mathrm{H}$ NMR $\left(400 \mathrm{MHz}, \mathrm{CDCl}_{3}\right)(\mathrm{ca} 0.04 \mathrm{mmol} / \mathrm{mL}) \delta 8.21(\mathrm{dd}, 1 \mathrm{H}, J=6.8,2.5 \mathrm{~Hz}, \mathrm{ArH}), 7.50$ (m, $2 \mathrm{H}, \mathrm{ArH}), 2.87\left(\mathrm{~s}, 3 \mathrm{H}, \mathrm{CH}_{3}\right), 1.10(\mathrm{~m}, 21 \mathrm{H}, \mathrm{TIPS}) .{ }^{13} \mathrm{C} \mathrm{NMR}\left(101 \mathrm{MHz}, \mathrm{CDCl}_{3}\right) \delta 166.8,140.3$, 138.0, 133.3, 131.7, 130.8, 119.1, 112.5, 66.9, 24.0, 18.5, 11.2. IR 2946 (w), 2867 (w), $2244(\mathrm{w}), 1649$ (m), $1562(\mathrm{w}), 1464(\mathrm{w}), 1326(\mathrm{w}), 1281(\mathrm{w}), 998(\mathrm{w}), 907(\mathrm{~s}), 884(\mathrm{w}), 763(\mathrm{w}), 728$ (s), $687(\mathrm{~s}), 647(\mathrm{~m})$. HRMS(ESI) calcd for $\mathrm{C}_{19} \mathrm{H}_{28} \mathrm{O}_{2} \mathrm{ISi}^{+}(\mathrm{M}+\mathrm{H})$ 443.0903, found 443.0893. The crystal structure of $\mathbf{5 d}$ has been deposited at the Cambridge Crystallographic Data Centre and allocated the deposition number: CCDC 863350. 


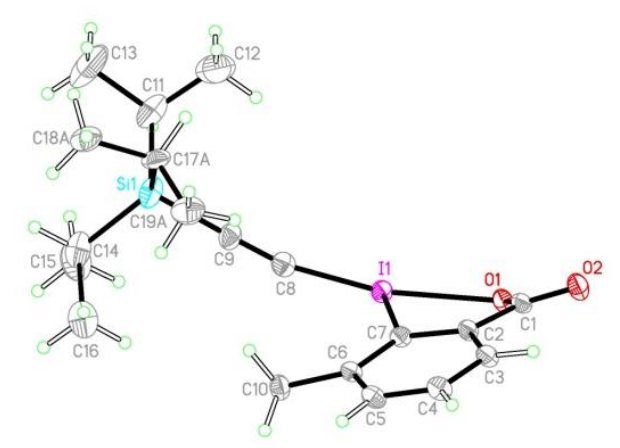

6-Methyl-2-iodosylbenzoic acid (13f)
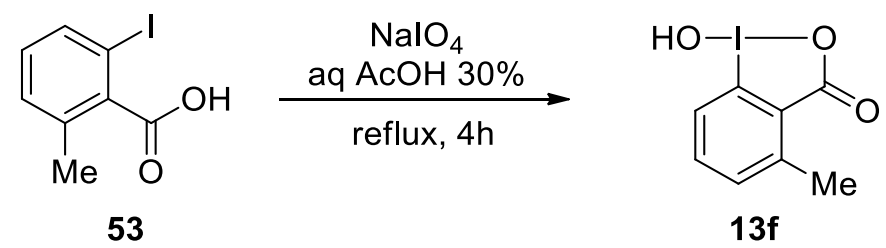

Following the reported procedure, ${ }^{13} \mathrm{NaIO}_{4}(2.57 \mathrm{~g}, 12.0 \mathrm{mmol}, 1.05$ equiv) and 2-iodo-6-methylbenzoic acid (53) (3.00 g, $11.5 \mathrm{mmol}, 1.00$ equiv) were suspended in $30 \%$ (v:v) aq. AcOH (105 mL). The mixture was vigorously stirred and refluxed for $4 \mathrm{~h}$. The reaction mixture was then diluted with cold water $(75 \mathrm{~mL})$ and allowed to cool to RT, protecting it from light. The crude product was collected by filtration, washed on the filter with ice water $(3 \times 5 \mathrm{~mL})$ and acetone $(3 \times 5 \mathrm{~mL})$, and air-dried in the dark to give the pure product 13f $(1.24 \mathrm{~g}, 4.46 \mathrm{mmol}, 80 \%)$ as a colorless solid. ${ }^{1} \mathrm{H}$ NMR (400 MHz, MeOD) $\delta 7.84$ (m, $\left.1 \mathrm{H}, \mathrm{ArH}\right), 7.76$ (m, $1 \mathrm{H}, \mathrm{ArH}), 7.54$ (m, $1 \mathrm{H}, \mathrm{ArH}), 2.78$ (s, $3 \mathrm{H}, \mathrm{Me})$.

6-Methyl-1-[(triisopropylsilyl)ethynyl]-1,2-benziodoxol-3(1H)-one (15f)

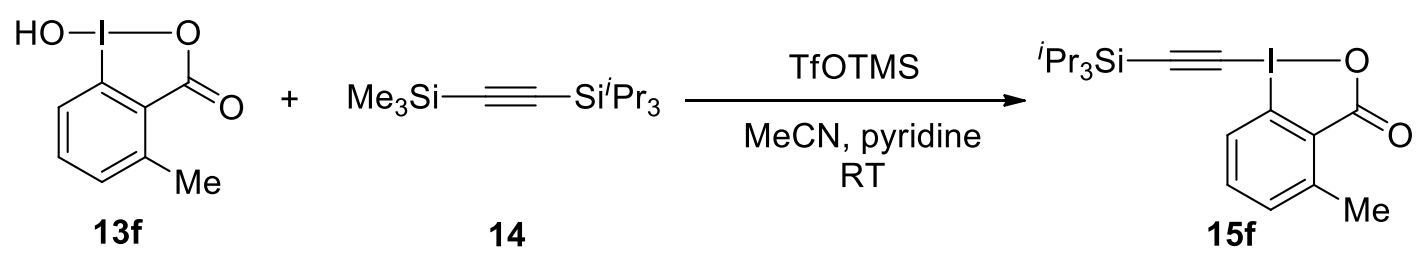

Trimethylsilyltriflate $(1.50 \mathrm{~mL}, 8.27 \mathrm{mmol}, 1.1$ equiv, freshly distilled) was added dropwise to a stirred solution of $\mathbf{1 3 f}$ ( $2.09 \mathrm{~g}, 7.52 \mathrm{mmol}, 1.0$ equiv) in acetonitrile (30 mL). After $20 \mathrm{~min}$, (trimethylsilyl)(triisopropylsilyl)acetylene (14) $(2.10 \mathrm{~g}, 8.27 \mathrm{mmol}, 1.1$ equiv) was then added dropwise, followed, after $20 \mathrm{~min}$, by the addition of pyridine $(667 \mu \mathrm{L}, 8.27 \mathrm{mmol}, 1.1$ equiv). The mixture was stirred $20 \mathrm{~min}$. The solvent was then removed under reduced pressure and the yellow crude oil was dissolved in dichloromethane $(150 \mathrm{~mL})$. The organic layer was washed with $1 \mathrm{M} \mathrm{HCl}(150 \mathrm{~mL})$ and the aqueous layer was extracted with $\mathrm{CH}_{2} \mathrm{Cl}_{2}$ $(150 \mathrm{~mL})$. The organic layers were combined, washed with a saturated solution of $\mathrm{NaHCO}_{3}(150 \mathrm{~mL})$, dried over $\mathrm{MgSO}_{4}$, filtered and the solvent was evaporated under reduced pressure. Recrystallization from acetonitrile and wash with cold acetonitrile afforded $\mathbf{1 5 f}(2.84 \mathrm{~g}, 6.60 \mathrm{mmol}, 88 \%)$ as colorless cristals. Mp: $123-125^{\circ} \mathrm{C} .{ }^{1} \mathrm{H}$ NMR (400 MHz, $\left.\mathrm{CDCl}_{3}\right) \delta 8.25$ (m, $\left.1 \mathrm{H}, \mathrm{ArH}\right), 7.53$ (d, $\left.2 \mathrm{H}, J=5.2 \mathrm{~Hz}, \mathrm{ArH}\right), 2.90$ (s, 3 $\left.\mathrm{H}, \mathrm{CH}_{3}\right), 1.15$ (m, $\left.21 \mathrm{H}, \mathrm{TIPS}\right) .{ }^{13} \mathrm{C}$ NMR $\left(101 \mathrm{MHz}, \mathrm{CDCl}_{3}\right) \delta 166.8,146.7,135.0,133.3,128.7,124.2$, 118.3, 113.3, 68.7, 22.4, 18.5, 11.2. IR 3055 (w), 2938 (m), $2873(\mathrm{~m}), 2865(\mathrm{~m}), 2244(\mathrm{w}), 2089(\mathrm{w}), 1626$ (s), $1612(\mathrm{~s}), 1586(\mathrm{~m}), 1550(\mathrm{~m}), 1450(\mathrm{~m}), 1382(\mathrm{w}), 1329(\mathrm{~m}), 1276(\mathrm{w}), 1253(\mathrm{w}), 1157(\mathrm{w}), 1076(\mathrm{w})$, 1018 (w), 998 (w), 911 (w), 884 (m), 846 (m), 817 (m), 770 (m), 706 (s), 679 (s), 649 (m). HRMS (ESI) calcd for $\mathrm{C}_{19} \mathrm{H}_{28} \mathrm{IO}_{2} \mathrm{Si}^{+}[\mathrm{M}+\mathrm{H}]^{+}$443.0898; found 443.0896. The crystal structure of $\mathbf{5 d}$ has been deposited at the Cambridge Crystallographic Data Centre and allocated the deposition number: CCDC 863349. 


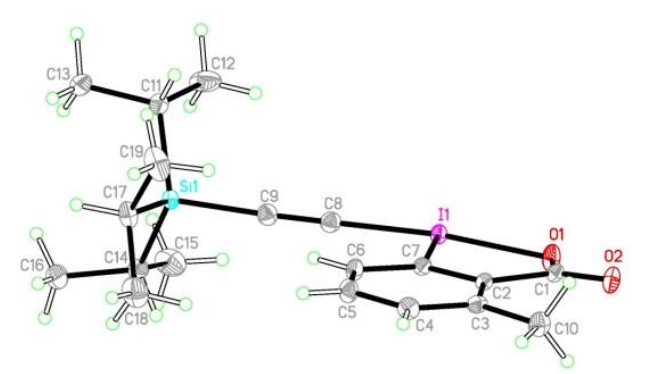

1-Chloro-1,3-dihydro-3,3-bis(trifluoromethyl)-1,2-benziodoxole (56)

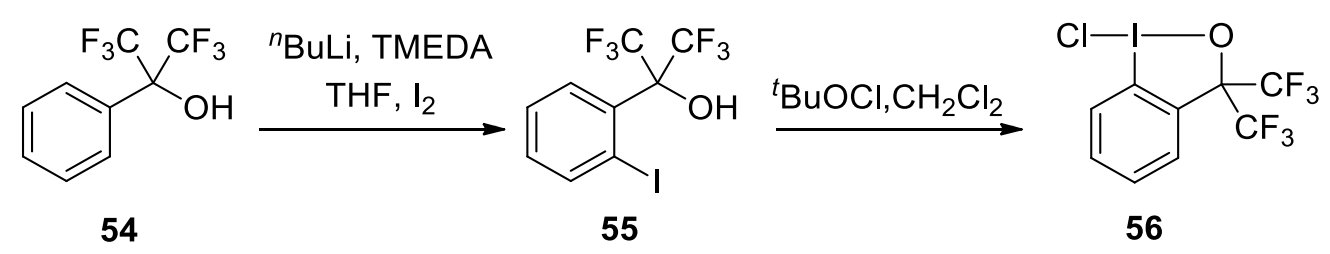

Following a reported procedure, ${ }^{16}$ TMEDA (distilled over KOH) $(0.63 \mathrm{~mL}, 4.1 \mathrm{mmol}, 0.2$ equiv) was added to a solution of ${ }^{n} \mathrm{BuLi}(2.5 \mathrm{M}$ in hexanes, $18.3 \mathrm{~mL}, 45.8 \mathrm{mmol}, 2.2$ equiv). After $15 \mathrm{~min}$, the cloudy solution was cooled to $0{ }^{\circ} \mathrm{C}$ and $54(3.5 \mathrm{~mL}, 21 \mathrm{mmol}, 1$ equiv) in THF $(3 \mathrm{~mL})$ was added dropwise. The reaction was stirred $30 \mathrm{~min}$ at $0{ }^{\circ} \mathrm{C}$ and then $18 \mathrm{~h}$ at RT. $\mathrm{I}_{2}(5.6 \mathrm{~g}, 22 \mathrm{mmol}, 1.06$ equiv) was then added portion wise at $0^{\circ} \mathrm{C}$ and the mixture stirred at $0^{\circ} \mathrm{C}$ for $30 \mathrm{~min}$ and $4 \mathrm{~h}$ at RT. The reaction was quenched with saturated $\mathrm{NH}_{4} \mathrm{Cl}$. $\mathrm{Et}_{2} \mathrm{O}(50 \mathrm{~mL})$ was added and the layers were separated. The aqueous layer was then extracted twice with $\mathrm{Et}_{2} \mathrm{O}(2 \times 50 \mathrm{~mL})$. The organic layers were combined, washed twice with saturated $\mathrm{NaS}_{2} \mathrm{O}_{3}(2 \times 50$ $\mathrm{mL}$ ), dried over $\mathrm{MgSO}_{4}$, filtered and reduced to afford $7.83 \mathrm{~g}$ of $\mathbf{5 5}$ as an orange oil which was used without further purification.

The crude oil was dissolved in wet $\mathrm{CH}_{2} \mathrm{Cl}_{2}(20 \mathrm{~mL})$ in the dark under air. ${ }^{t} \mathrm{BuOCl}(2.6 \mathrm{~mL}, 22 \mathrm{mmol}, 1.05$ equiv) was then added dropwise at $0{ }^{\circ} \mathrm{C}$. After $30 \mathrm{~min}$, the resulting suspension was filtered to afford $\mathbf{5 6}$ (3.52 g, $8.70 \mathrm{mmol}, 42 \%)$ as a yellow oil. The mother liquors were carefully reduced to one third and filtered to afford $56(2.33 \mathrm{~g}, 5.76 \mathrm{mmol}, 28 \%)$ as a yellow solid. Combined yield: $70 \%$. Mp $167-169^{\circ} \mathrm{C} .{ }^{1} \mathrm{H}$ NMR (400 MHz, $\left.\mathrm{CDCl}_{3}\right) \delta 8.09(\mathrm{~d}, 1 \mathrm{H}, J=8.4 \mathrm{~Hz}, \mathrm{ArH}), 7.85(\mathrm{~m}, 1 \mathrm{H}, \mathrm{ArH}), 7.73(\mathrm{~m}, 2 \mathrm{H}, \mathrm{ArH}) .{ }^{13} \mathrm{C}$ NMR $\left(101 \mathrm{MHz}, \mathrm{CDCl}_{3}\right) \delta 133.8,132.1,131.6,129.7,128.5,122.8$ (q, $\left.289 \mathrm{~Hz}\right), 113.4,84.8$. Consistent with reported values. ${ }^{17}$

\section{1-Hydroxy-3,3-bis(trifluoromethyl)-3-(1H)-1,2-benziodoxole 13g}

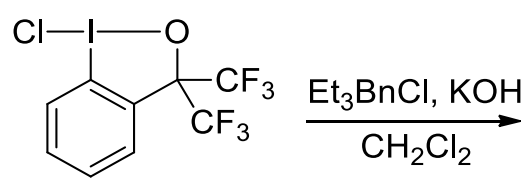

57<smiles>OI1OC(C(F)(F)F)(C(F)(F)F)c2ccccc21</smiles>

$13 \mathrm{~g}$

Following a preported procedure, ${ }^{18} \mathrm{Et}_{3} \mathrm{BnNCl}(83 \mathrm{mg}, 0.36 \mathrm{mmol}, 0.05$ equiv) was added to a stirring solution of $\mathbf{5 6}$ (3.00 g, $7.41 \mathrm{mmol}, 1$ equiv) in $\mathrm{CH}_{2} \mathrm{Cl}_{2}(50 \mathrm{~mL})$ and $\mathrm{KOH}(415 \mathrm{mg}, 7.41 \mathrm{mmol}, 1$ equiv) in water $(8 \mathrm{~mL})$. The reaction was stirred for $3 \mathrm{~h} 30$ under air. The organic layer was separated and dried over

\footnotetext{
${ }^{16}$ Perozzi, E. F.; Michalak, R. S.; Figuly, G. D.; Stevenson, W. H.; Dess, D. B.; Ross, M. R.; Martin, J. C. J. Org. Chem. 1981, 46, 1049.

${ }^{17}$ Cvengros, J.; Stolz, D.; Togni, A. Synthesis 2009, 2818.

${ }^{18}$ Blake, A. J.; Novak, A.; Davies, M.; Robinson, R. I.; Woodward, S. Synth. Commun. 2009, 39, 1065
} 
$\mathrm{MgSO}_{4}$. The resulting solid was purified over a silica plug with EtOAc,then recristallized in EtOAC and washed with pentane to afford $13 \mathrm{~g}(1.24 \mathrm{~g}, 3.21 \mathrm{mmol}, 43 \%)$ as a colorless solid. The mother liquors were reduced and recristallized in EtOAc to afford a second batch of $\mathbf{1 3 g}(279 \mathrm{mg}, 0.723 \mathrm{mmol}, 10 \%)$ as a colorless solid. Combined yield: 53\%. ${ }^{1} \mathrm{H}$ NMR (400 MHz, DMSO) $\delta 7.96$ (m, $\left.2 \mathrm{H}, \mathrm{ArH}\right), 7.73(\mathrm{~m}, 2 \mathrm{H}$, ArH). ${ }^{13} \mathrm{C}$ NMR (101 MHz, DMSO) $\delta 133.3,131.0,130.8,128.9,127.9,123.4(\mathrm{q}, J=290 \mathrm{~Hz}), 117.2,83.7$ (m). IR $1464(\mathrm{w}), 1435(\mathrm{w}), 1290$ (w), 1263 (m), 1185 (s), 1139 (s), 1103 (m), 1041 (w), 1021 (w), 952 (s), $760(\mathrm{~m}), 730(\mathrm{~m}), 692(\mathrm{~m})$.

\section{1-[(Triisopropylsilyl)ethynyl]-3,3-bis(trifluoromethyl)-3(1H)-1,2-benziodoxole $15 \mathrm{~g}$}

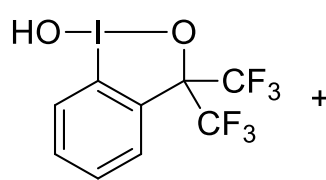

$13 \mathrm{~g}$

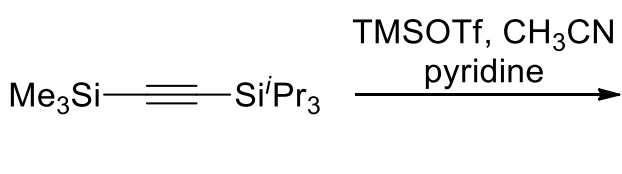

14

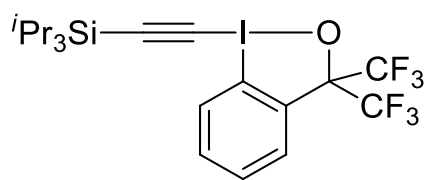

$15 \mathrm{~g}$

TMSOTf ( $310 \mu \mathrm{L}, 1.71 \mathrm{mmol}, 1.1$ equiv) was added to $\mathbf{1 3 g}$ (600 mg, $1.55 \mathrm{mmol}, 1.0$ equiv) in $\mathrm{CH}_{2} \mathrm{Cl}_{2}(20$ $\mathrm{mL})$ at RT. After $20 \mathrm{~min}$, the solution was reduced and the resulting oil was dissolved in $\mathrm{CH}_{3} \mathrm{CN}(30 \mathrm{~mL})$. (trimethylsilyl)(triiso-propylsilyl)acetylene (14) $(514 \mathrm{mg}, 1.71 \mathrm{mmol}, 1.1$ equiv) was added and after $20 \mathrm{~min}$ pyridine ( $76 \mu \mathrm{L}, 0.94 \mathrm{mmol}, 0.6$ equiv) was added. The reaction was then reduced under vacuum, dissolved in $\mathrm{Et}_{2} \mathrm{O}$ and filtered over a silica plug (eluant $\mathrm{Et}_{2} \mathrm{O}$ ). The resulting solid was purified by column chromatography (PET/Et $2 \mathrm{O} 95 / 5)$ to afford $15 \mathrm{~g}(816 \mathrm{mg}, 1.48 \mathrm{mmol}, 95 \%)$ as a colorless solid. Rf (PET/Et $2 \mathrm{O}$ 95/5): 0.4. $\mathrm{Mp} 131-132^{\circ} \mathrm{C} .{ }^{1} \mathrm{H}$ NMR $\left(400 \mathrm{MHz}, \mathrm{CDCl}_{3}\right)(c a 0.10 \mathrm{mmol} / \mathrm{mL}) \delta 8.36(\mathrm{dd}, 1 \mathrm{H}, J$ = 7.9, $1.7 \mathrm{~Hz}, \operatorname{ArH}), 7.84(\mathrm{~d}, 1 \mathrm{H}, J=6.7 \mathrm{~Hz}, \operatorname{ArH}), 7.68(\mathrm{~m}, 2 \mathrm{H}, \mathrm{ArH}), 1.15(\mathrm{~m}, 21 \mathrm{H}, \mathrm{TIPS}) .{ }^{13} \mathrm{C}$ NMR $\left(101 \mathrm{MHz}, \mathrm{CDCl}_{3}\right) \delta 132.7,131.1,129.9,129.9(\mathrm{~m}), 128.2,123.6$ (q, $\left.288 \mathrm{~Hz}\right), 112.1,110.8,81.4(\mathrm{~m}), 69.7$, 18.5, 11.2. IR $2947(\mathrm{~m}), 2868(\mathrm{~m}), 2249(\mathrm{w}), 1566(\mathrm{w}), 1465(\mathrm{~m}), 1438(\mathrm{w}), 1387(\mathrm{w}), 1264(\mathrm{~s}), 1218(\mathrm{~m})$, $1184(\mathrm{~s}), 1149$ (s), $1071(\mathrm{w}), 994(\mathrm{w}), 951$ (s), 910 (m), 873 (w), 732 (s), 696 (s), 655 (s), 655 (s). HRMS(ESI) calcd for $\mathrm{C}_{20} \mathrm{H}_{26} \mathrm{OF}_{6} \mathrm{ISi}^{+}(\mathrm{M}+\mathrm{H})$ 551.0702, found 551.0723.

\section{2-[(Triisopropylsilylethynyl)(trifluoromethanesulfonyloxy)iodo]benzoic acid (15h)}

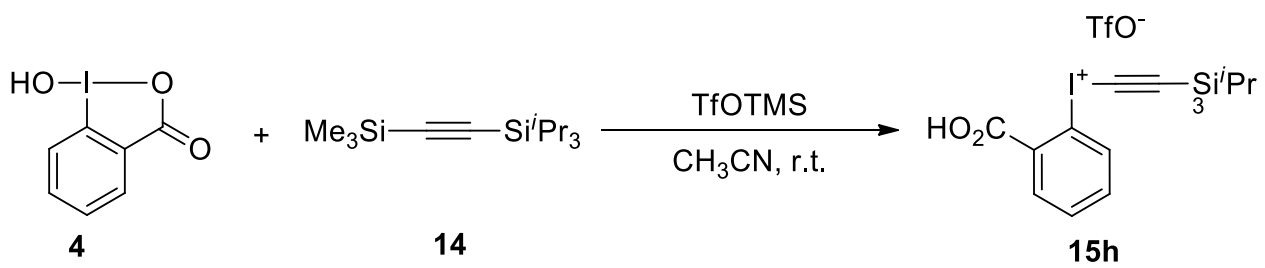

Following a reported procedure, ${ }^{7}$ trimethylsilyltriflate $(301 \mu \mathrm{L}, 1.65 \mathrm{mmol}, 1.1$ equiv, freshly distilled) was added dropwise to a stirred solution of 2-iodosylbenzoic acid (4) (400 mg, $1.65 \mathrm{mmol}, 1.0$ equiv) in DCM (5 $\mathrm{mL}$ ). After $1 \mathrm{~h}$, (trimethylsilyl)(triisopropylsilyl)acetylene (14) (422 mg, $1.65 \mathrm{mmol}, 1.1$ equiv,) was added dropwise. The mixture was stirred $5 \mathrm{~h}$. The solvent was then removed under reduced pressure and the yellow crude oil was crystallized in $\mathrm{Et}_{2} \mathrm{O} / \mathrm{h}$ exanes $1 / 1$ to give $\mathbf{1 5 h}(822 \mathrm{mg}, 1.42 \mathrm{mmol}, 95 \%)$. An analytical pure sample was obtained by recristallization of a saturated solution in DCM by addition of $\mathrm{Et}_{2} \mathrm{O} / \mathrm{hexanes}^{1 / 1}$. Mp (Dec.) $156-158^{\circ} \mathrm{C} .{ }^{1} \mathrm{H}$ NMR $\left(400 \mathrm{MHz}, \mathrm{CDCl}_{3}\right) \delta 8.41$ (dd, $\left.1 \mathrm{H}, J=7.5,1.7 \mathrm{~Hz}, \mathrm{ArH}\right), 8.35(\mathrm{dd}, 1 \mathrm{H}, J$ $=8.3,1.0 \mathrm{~Hz}, \mathrm{ArH}), 7.92(\mathrm{ddd}, J=8.1,7.4,1.7 \mathrm{~Hz}, \mathrm{ArH}), 7.87$ (td, $1 \mathrm{H}, J=7.4,1.1 \mathrm{~Hz}, \mathrm{ArH}), 1.20(\mathrm{~m}, 3 \mathrm{H}$, $\mathrm{CH}), 1.13\left(\mathrm{~m}, 18 \mathrm{H}, \mathrm{CH}_{3}\right) .{ }^{13} \mathrm{C} \mathrm{NMR}\left(101 \mathrm{MHz}, \mathrm{CDCl}_{3}\right) \delta 170.9,137.7,133.6,132.3,128.8,125.9,121.9$, $120.0(\mathrm{q}, J=319 \mathrm{~Hz}), 114.5,48.2,18.4,11.1$. IR $3476(\mathrm{w}), 3086(\mathrm{w}), 2947(\mathrm{~m}), 2868(\mathrm{~m}), 2509(\mathrm{w}), 2255$ (w), $1645(\mathrm{~m}), 1590(\mathrm{w}), 1464(\mathrm{w}), 1440$ (w), 1285 (s), 1226 (s), 1169 (s), 1072 (w), 1026 (s), 999 (m), 912 
(m), 883 (m), 804 (w), 713 (s), 680 (s), 641 (s). HRMS(ESI) calcd for $\mathrm{C}_{18} \mathrm{H}_{26} \mathrm{O}_{2} \mathrm{ISi}$ (M-OTf) 429.0747, found 429.0736 .

\section{1-Hydroxy-3,3-dimethyl-3-(1H)-1,2-benziodoxole (16)}

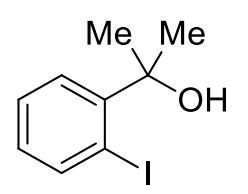

58

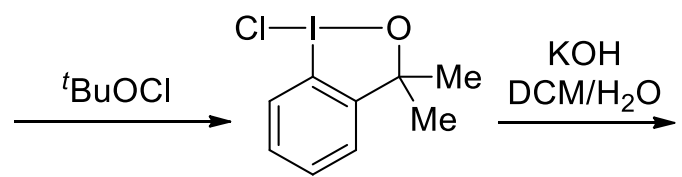

59

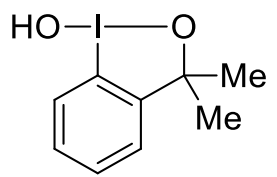

16

Following a reported procedure, ${ }^{19}{ }^{t} \mathrm{BuOCl}(1.0 \mathrm{~mL}, 8.4 \mathrm{mmol}, 1.1$ equiv) was added to 58 (1.95 g, 7.44 mmol, 1 equiv) at RT under air in the dark. The reaction was stirred for $30 \mathrm{~min}$ at RT. Solvent was removed under vacuum and the resulting solid dried overnight under high vacuum to afford $\mathbf{5 9}(2.02 \mathrm{~g}, 6.82 \mathrm{mmol}$, 92\%) as a solid. ${ }^{1} \mathrm{H} \mathrm{NMR}\left(400 \mathrm{MHz}, \mathrm{CDCl}_{3}\right) \delta 8.03(\mathrm{dd}, 1 \mathrm{H}, J=8.1,1.3 \mathrm{~Hz}, \mathrm{ArH}), 7.55(\mathrm{~m}, 2 \mathrm{H}, \mathrm{ArH})$, 7.17 (dd, $1 \mathrm{H}, J=7.3,1.7 \mathrm{~Hz}, \mathrm{ArH}), 1.55$ (s, $6 \mathrm{H}, \mathrm{Me}$ ).

The solid 58 (2.02 g, $6.82 \mathrm{mmol}, 1$ equiv) was then dissolved in $\mathrm{CH}_{2} \mathrm{Cl}_{2}(30 \mathrm{~mL})$ and $\mathrm{KOH}(382 \mathrm{mg}, 6.82$ mmol, 1 equiv) in water $(3 \mathrm{~mL})$ was added. After $3 \mathrm{~h}$, the organic layer was separated, dried over $\mathrm{MgSO}_{4}$, filtered over $\mathrm{MgSO}_{4}$ and concentrated. The resulting solid was recristalized in EtOAc, washed with hexanes and dried under vacuum to afford $16(1.11 \mathrm{~g}, 3.99 \mathrm{mmol}, 59 \%)$ as a colorless solid. Mp $241-243^{\circ} \mathrm{C} .{ }^{1} \mathrm{H}$ NMR (400 MHz, DMSO) $\delta 7.79$ (d, $1 \mathrm{H}, J=7.9 \mathrm{~Hz}, \operatorname{ArH}), 7.52(\mathrm{~m}, 2 \mathrm{H}$, ArH), 7.36 (d, $1 \mathrm{H}, J=7.4 \mathrm{~Hz}$, $\mathrm{ArH}), 1.36$ (s, $6 \mathrm{H}, \mathrm{Me}) .{ }^{13} \mathrm{C}$ NMR $\left(101 \mathrm{MHz}, \mathrm{CDCl}_{3}\right) \delta 150.0,129.7,129.2,127.0,126.2,115.3,79.4,30.2$. IR 1569 (w), 1464 (w), 1443 (w), 1263 (s), 1185 (s), 1140 (s), 1110 (s), 1020 (w), 952 (s), 761 (m), 730 (m), $690(\mathrm{~m}), 643(\mathrm{~s})$.

\section{1-[(Triisopropylsilyl)ethynyl]-3,3-dimethyl-3(1H)-1,2-benziodoxole (17)}

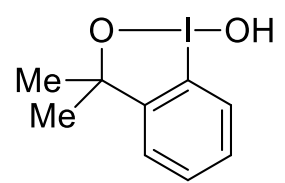

16

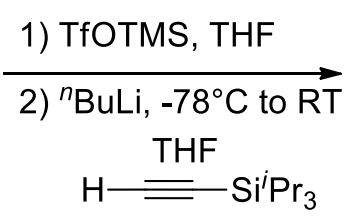

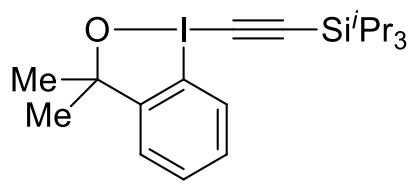

17

Trimethylsilyltriflate ( $250 \mu \mathrm{L}, 1.38 \mathrm{mmol}, 1$ equiv) was added to a stirring solution of $\mathbf{1 6}$ (408 $\mathrm{mg}, 1.38$ mmol, 1 equiv) in THF ( $40 \mathrm{~mL})$ at RT. The solution was stirred at RT for $20 \mathrm{~min}$ and then cooled to $-78^{\circ} \mathrm{C}$. In the meantime, ${ }^{n} \mathrm{BuLi}(2.5 \mathrm{M}$ in hexanes, $550 \mu \mathrm{L}, 1.38 \mathrm{mmol}, 1$ equiv) was added to a stirring solution of triisopropylacetylene $\left(310 \mu \mathrm{L}, 1.38 \mathrm{mmol}, 1\right.$ equiv) in THF $(10 \mathrm{~mL})$ at $-78^{\circ} \mathrm{C}$. The solution was stirred for $30 \mathrm{~min}$ at $-78^{\circ} \mathrm{C}$ and then added via cannula to the first solution. The reaction was stirred for $1 \mathrm{~h}$ at $-78^{\circ} \mathrm{C}$, warmed to RT and stirred $4 \mathrm{~h}$. The reaction was quenched with saturated $\mathrm{NH}_{4} \mathrm{Cl}(20 \mathrm{~mL})$. The layers were separated and the aqueous layers were extracted with $\mathrm{CH}_{2} \mathrm{Cl}_{2}(20 \mathrm{~mL})$. The organic layers were combined, washed with brine, dried over $\mathrm{MgSO}_{4}$, filtered and reduced under vacuum. The resulting oil was then purified by column chromatography (PET/Et ${ }_{2} \mathrm{O}$ 6/4) to afford 17 (524 mg, $\left.1.18 \mathrm{mmol}, 86 \%\right)$ as a yellow oil which cristalize at $-18^{\circ} \mathrm{C}$. $\mathrm{R}_{\mathrm{f}} \mathrm{PET}_{2} \mathrm{Et}_{2} \mathrm{O}$ 6/4: 0.15. Mp $59-61{ }^{\circ} \mathrm{C} .{ }^{1} \mathrm{H}$ NMR $\left(400 \mathrm{MHz}, \mathrm{CDCl}_{3}\right)($ ca 0.16 $\mathrm{mmol} / \mathrm{mL}) \delta 8.23(\mathrm{dd}, 1 \mathrm{H}, J=8.2,0.9 \mathrm{~Hz}, \mathrm{ArH}), 7.52(\mathrm{td}, 1 \mathrm{H}, J=7.3,1.0 \mathrm{~Hz}, \mathrm{ArH}), 7.41(\mathrm{ddd}, 1 \mathrm{H}, J=$ 8.6, 7.2, $1.5 \mathrm{~Hz}, \mathrm{ArH}), 7.35$ (dd, $1 \mathrm{H}, J=7.5,1.5 \mathrm{~Hz}, \mathrm{ArH}), 1.44$ (s, $6 \mathrm{H}, \mathrm{Me}), 1.12$ (s, $21 \mathrm{H}, \mathrm{TIPS}) .{ }^{13} \mathrm{C}$ NMR $\left(101 \mathrm{MHz}, \mathrm{CDCl}_{3}\right) \delta 148.0,130.4,129.2,127.4,126.5,111.0,105.8,80.8,75.7,31.5,18.6,11.4$. IR 2945 (m), $2864(\mathrm{~m}), 2064$ (w), $1690(\mathrm{w}), 1562$ (w), $1462(\mathrm{~m}), 1436(\mathrm{~m}), 1355(\mathrm{w}), 1244(\mathrm{w}), 1162(\mathrm{w}), 1116$

\footnotetext{
${ }^{19}$ Moss, R. A.; Wilk, B.; Kroghjespersen, K.; Blair, J. T.; Westbrook, J. D. J. Am. Chem. Soc. 1989, 111, 250
} 
(w), 1073 (w), $999(\mathrm{~m}), 968(\mathrm{~m}), 883(\mathrm{~m}), 756$ (m), 691 (s). HRMS(ESI) calcd for $\mathrm{C}_{20} \mathrm{H}_{32} \mathrm{OISi}^{+}(\mathrm{M}+\mathrm{H})$ 443.1267, found 443.1276 .

\section{Evaluation of substituted benziodoxolones}

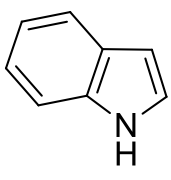

6

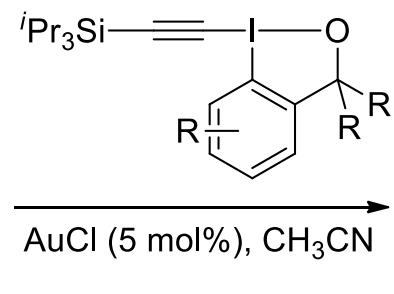

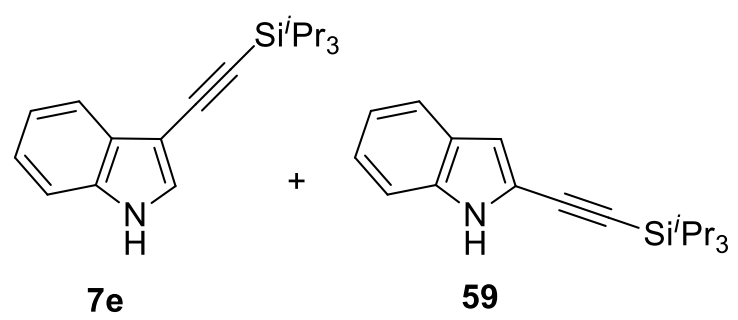

59

$\mathrm{AuCl}$ (2.3 mg, $0.010 \mathrm{mmol}, 0.1$ equiv) was added to a stirring solution of the corresponding benziodoxolone (0.120 mmol, 1.2 equiv) and indole $(11.7 \mathrm{mg}, 0.100 \mathrm{mmol}, 1.0$ equiv) in $0.025 \mathrm{M}$ solution of undecylcyanide ${ }^{20}$ in $\mathrm{CH}_{3} \mathrm{CN}^{1}(2 \mathrm{~mL})$ under air. After $14 \mathrm{~h}, \mathrm{GC} / \mathrm{MS}$ analysis afforded the yield.

For entry 8 in Table 3: $\mathrm{Et}_{2} \mathrm{O}(10 \mathrm{~mL})$ was added, the organic layer was washed twice with $0.1 \mathrm{M} \mathrm{NaOH}(15$ $\mathrm{mL})$. The aqueous layers were combined and extracted with $\mathrm{Et}_{2} \mathrm{O}(20 \mathrm{~mL})$. The organic layers were combined, washed with saturated $\mathrm{NaHCO}_{3}(20 \mathrm{~mL})$, brine $(20 \mathrm{~mL})$, dried with $\mathrm{MgSO}_{4}$ and concentrated under reduced pressure. Purification by column chromatography (pentane/Et $2 \mathrm{O}$ 95/5) afforded 7e (12 mg, $0.040 \mathrm{mmol}, 40 \%)$ as a colorless oil and $59(4 \mathrm{mg}, 0.01 \mathrm{mmol}, 14 \%)$ as a colorless oil. Values of 7e consistent with reported data.2 59: ${ }^{1} \mathrm{H}$ NMR $\left(400 \mathrm{MHz}, \mathrm{CDCl}_{3}\right) \delta 8.17$ (br s, $\left.1 \mathrm{H}, \mathrm{NH}\right), 7.57$ (dd, $1 \mathrm{H}, J=$ 8.0, $0.8 \mathrm{~Hz}, \mathrm{ArH}), 7.31$ (dd, $1 \mathrm{H}, J=8.2,0.9 \mathrm{~Hz}, \mathrm{ArH}), 7.22(\mathrm{~m}, 1 \mathrm{H}, \mathrm{ArH}), 7.11$ (m, $1 \mathrm{H}, \mathrm{ArH}), 6.78$ (dd, 1 $\mathrm{H}, J=2.1,0.9 \mathrm{~Hz}, \mathrm{ArH}), 1.14$ (m, $21 \mathrm{H}, \mathrm{TIPS}) .{ }^{13} \mathrm{C} \mathrm{NMR}\left(101 \mathrm{MHz}, \mathrm{CDCl}_{3}\right) \delta 135.8,127.6,123.6,120.9$, 120.5, 119.0, 110.7, 109.2, 98.8, 95.0, 18.7, 11.3. IR v $3422(w), 2945(w), 2866(w), 2348(w), 2154(w)$, 1457 (w), 1347 (w), 994 (w), 884 (w), 795 (w), 721 (m), 672 (s). HRMS(ESI) calcd for $\mathrm{C}_{19} \mathrm{H}_{28} \mathrm{NSi}^{+}(\mathrm{M}+\mathrm{H})^{+}$ 298.1991 , found 298.1982 .

\section{Mechanistic Investigations}

\section{Competitive experiments}<smiles>N#Cc1ccc2[nH]ccc2c1</smiles>

3 eq<smiles>c1ccc2[nH]ccc2c1</smiles>

3 eq

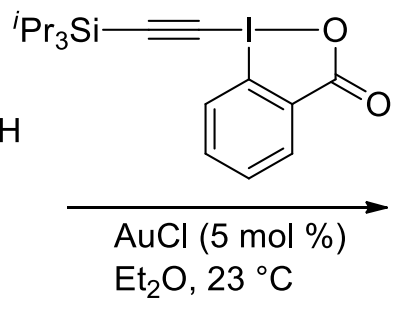

$\mathrm{Et}_{2} \mathrm{O}, 23^{\circ} \mathrm{C}$

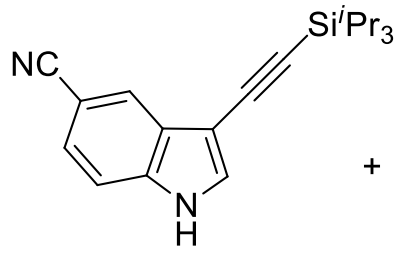

$25 \%$

22

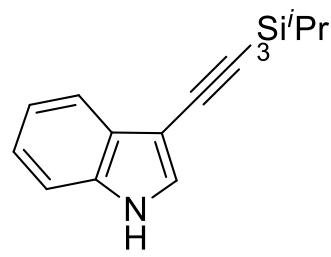

$74 \%$

$7 e$

$\mathrm{AuCl}(2.3 \mathrm{mg}, 0.010 \mathrm{mmol}, 0.05$ equiv) was added to a stirring solution of TIPS-EBX (1) (86 mg, 0.20 mmol, 1.0 equiv), indole (6) (70 mg, $0.60 \mathrm{mmol}, 3.0$ equiv) and 5-cyanoindole (21) ( $85 \mathrm{mg}, 0.60 \mathrm{mmol}, 3.0$ equiv) in $\mathrm{Et}_{2} \mathrm{O}^{1}(4 \mathrm{~mL})$ under air. The reaction was sealed and stirred at room temperature for $16 \mathrm{~h}$. $\mathrm{Et}_{2} \mathrm{O}(10$ $\mathrm{mL}$ ) was added, the organic layer was washed twice with $0.1 \mathrm{M} \mathrm{NaOH}(15 \mathrm{~mL})$. The aqueous layers were

\footnotetext{
${ }^{20}$ Undecylcyanide was used as internal reference due to its solubility in a wide range of solvent.
} 
combined and extracted with $\mathrm{Et}_{2} \mathrm{O}(20 \mathrm{~mL})$. The organic layers were combined, washed with saturated $\mathrm{NaHCO}_{3}(20 \mathrm{~mL})$, brine $(20 \mathrm{~mL})$, dried with $\mathrm{MgSO}_{4}$ and concentrated under reduced pressure. Purification by column chromatography (pentane/ $\left.\mathrm{Et}_{2} \mathrm{O} 9 / 1\right)$ afforded a mixture of 7e (44 mg, $\left.0.15 \mathrm{mmol}, 74 \%\right)$ and 6 (43 $\mathrm{mg}, 0.37 \mathrm{mmol}, 61 \%$ recovered) as a yellow oil as well as 22 (16 mg, $0.049 \mathrm{mmol}, 25 \%)$ and 21 (79 mg, $0.54 \mathrm{mmol}, 91 \%$ recovered) as an orange amorphous solid. NMR consistent with previously reported data. ${ }^{2}$

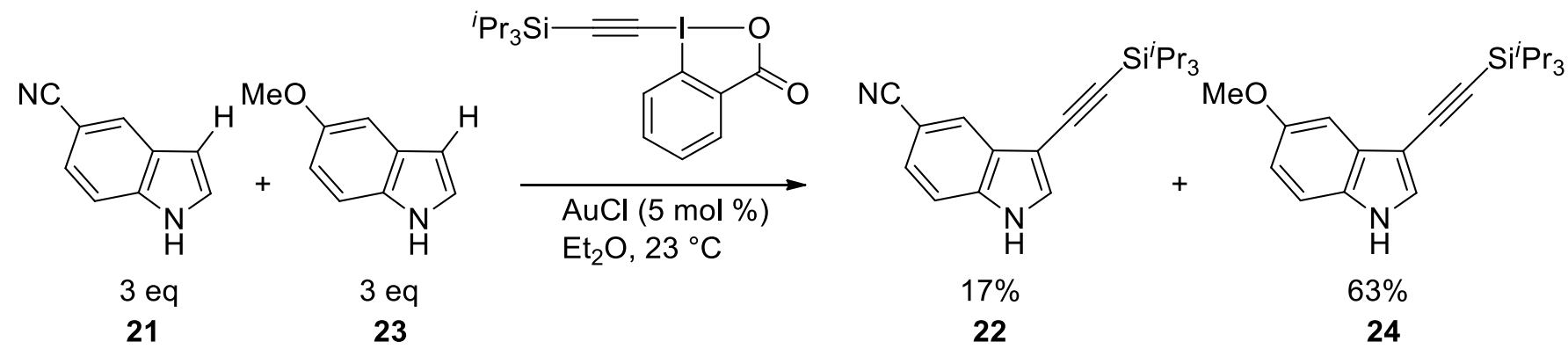

$\mathrm{AuCl}$ (2.3 mg, $0.010 \mathrm{mmol}, 0.05$ equiv) was added to a stirring solution of TIPS-EBX (1) (86 mg, 0.20 mmol, 1.0 equiv), 5-methoxyindole (23) (88 mg, $0.60 \mathrm{mmol}, 3.0$ equiv) and 5-cyanoindole (21) ( $85 \mathrm{mg}, 0.60$ mmol, 3.0 equiv) in $\mathrm{Et}_{2} \mathrm{O}^{1}(4 \mathrm{~mL})$ under air. The reaction was sealed and stirred at room temperature for 16 h. $\mathrm{Et}_{2} \mathrm{O}(10 \mathrm{~mL})$ was added, the organic layer was washed twice with $0.1 \mathrm{M} \mathrm{NaOH}(15 \mathrm{~mL})$. The aqueous layers were combined and extracted with $\mathrm{Et}_{2} \mathrm{O}(20 \mathrm{~mL})$. The organic layers were combined, washed with saturated $\mathrm{NaHCO}_{3}(20 \mathrm{~mL})$, brine $(20 \mathrm{~mL})$, dried with $\mathrm{MgSO}_{4}$ and concentrated under reduced pressure. Purification by column chromatography (pentane/ $\mathrm{Et}_{2} \mathrm{O} 9 / 1$ to 7/3) afforded a mixture of 24 (41 mg, 0.13 mmol, 63\%) and 23 (60 mg, $0.41 \mathrm{mmol}, 68 \%$ recovered) as a yellow oil as well as 22 (11 $\mathrm{mg}, 0.034 \mathrm{mmol}$, $17 \%$ ) and 21 (79 mg, $0.54 \mathrm{mmol}, 91 \%$ recovered) as an orange amorphous solid. NMR consistent with previously reported data. $^{2}$

\section{[3- $\left.{ }^{2} \mathbf{H}\right]$ Indole (25)}

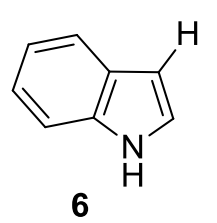

a) $0.01 \mathrm{M} \mathrm{DCl}$ in $\mathrm{D}_{2} \mathrm{O}$

b) $\mathrm{Et}_{2} \mathrm{O} / \mathrm{H}_{2} \mathrm{O}$

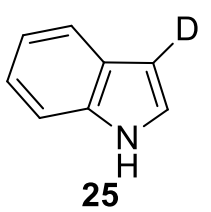

Following a reported procedure, ${ }^{21}$ indole (6) $(2.0 \mathrm{~g}, 0.017 \mathrm{mmol}, 1$ equiv) was stirred in a $0.01 \mathrm{M}$ solution of $\mathrm{DCl}$ in $\mathrm{D}_{2} \mathrm{O}(20 \mathrm{~mL})$ at $60^{\circ} \mathrm{C}$ for $4 \mathrm{~h}$ under nitrogen. The reaction was cooled and extracted three times with dry $\mathrm{Et}_{2} \mathrm{O}(20 \mathrm{~mL})$. The organic layers were combined, dried over $\mathrm{MgSO}_{4}$ and filtered and reduced under vacuum. The resulting oil was triturated in pentane to afford a colorless solid. The solid $\left(\left[1,3-{ }^{2} \mathrm{H}\right]\right.$ Indole $)$ was dissolved in a mixture of $\mathrm{Et}_{2} \mathrm{O}(20 \mathrm{~mL})$ and $\mathrm{H}_{2} \mathrm{O}(10 \mathrm{~mL})$ and stirred for $2 \mathrm{~h}$ at RT. The layers were separated and the aqueous layer extracted twice with $\mathrm{Et}_{2} \mathrm{O}(20 \mathrm{~mL})$. The organic layers were combined, dried over $\mathrm{MgSO}_{4}$ and filtered and reduced under vacuum. Crystallization in heptanes affords 25 (1.8 g, $0.015 \mathrm{mmol}, 88 \%)$. The ${ }^{1} \mathrm{H}$ NMR peak at $6.48 \mathrm{ppm}$ completely disappeared compared to indole indication more than $95 \%$ incorporation.

\section{$\left[3-{ }^{2} \mathrm{H}\right]$ Indole (25) and [3-H]Indole (6) competitive experiment}

\footnotetext{
${ }^{21}$ Ibacetalizana, J. S. L.; Jackson, A. H.; Prasitpan, N.; Shannon, P. V. R. J. Chem. Soc. Perkin Trans. 2 1987, 1221.
} 

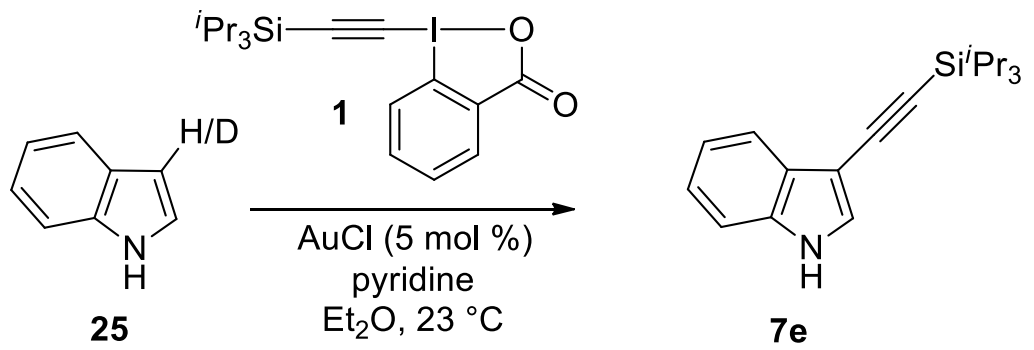

$\mathrm{AuCl}$ (2.3 mg, $0.010 \mathrm{mmol}, 0.05$ equiv) was added to a stirring solution of TIPS-EBX (1) (51 mg, 0.12 mmol, 0.6 equiv), pyridine ( $16 \mu \mathrm{L}, 0.40 \mathrm{mmol}, 2$ equiv), and indole 25 (23.4 mg, $0.200 \mathrm{mmol}, 1.0$ equiv, $36 \% \mathrm{D}$ incoporation) in $\mathrm{Et}_{2} \mathrm{O} 1(4 \mathrm{~mL})$ under air. The reaction was sealed and stirred at room temperature for $15 \mathrm{~h}$. $\mathrm{Et}_{2} \mathrm{O}(10 \mathrm{~mL})$ was added, the organic layer was washed twice with $0.1 \mathrm{M} \mathrm{NaOH}(15 \mathrm{~mL})$. The aqueous layers were combined and extracted with $\mathrm{Et}_{2} \mathrm{O}(20 \mathrm{~mL})$. The organic layers were combined, washed with saturated $\mathrm{NaHCO}_{3}(20 \mathrm{~mL})$, brine $(20 \mathrm{~mL})$, dried with $\mathrm{MgSO}_{4}$ and concentrated under reduced pressure. The crude mixture indicates that the remaining indole has an incorporation level of $47 \%$ (average of three reactions). When the reaction is carried out using the same procedure without TIPS-EBX (1) the remaining indole has an incorporation level of $46 \%$ (average of three reactions).

\section{3-((Triisopropylsilyl)ethynyl)-1H-indole (7e)}

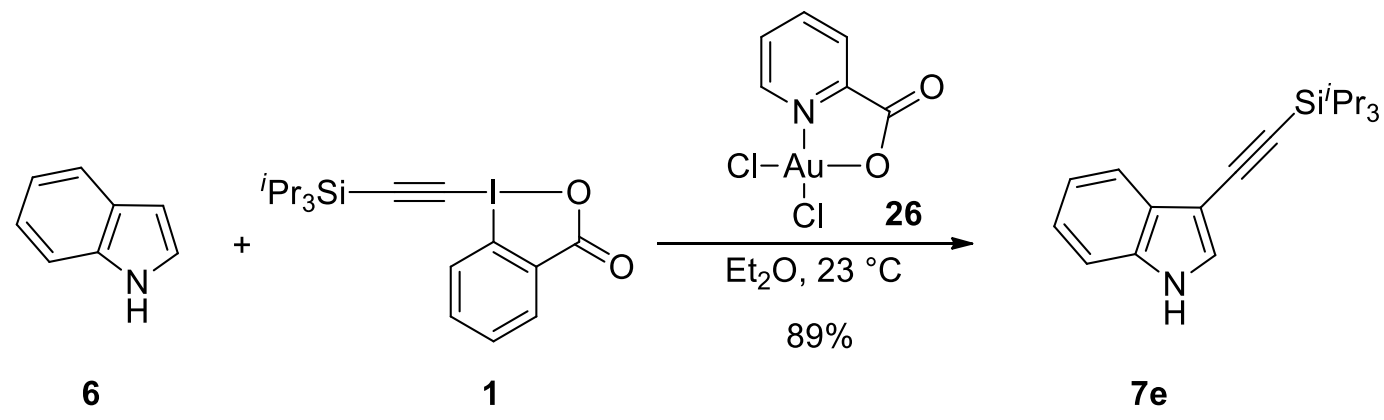

Gold 2-pyridinecarboxylate dichloride (26) $(3.9 \mathrm{mg}, 0.010 \mathrm{mmol}, 0.05$ equiv) was added to a stirring solution of TIPS-EBX (1) (103 mg, $0.240 \mathrm{mmol}, 1.2$ equiv), indole (6) (23.4 mg, $0.200 \mathrm{mmol}, 1.0$ equiv) in $\mathrm{Et}_{2} \mathrm{O} 1(4 \mathrm{~mL})$ under air. The reaction was sealed and stirred at room temperature for $15 \mathrm{~h}$. $\mathrm{Et}_{2} \mathrm{O}(10 \mathrm{~mL})$ was added, the organic layer was washed twice with $0.1 \mathrm{M} \mathrm{NaOH}(15 \mathrm{~mL})$. The aqueous layers were combined and extracted with $\mathrm{Et}_{2} \mathrm{O}(20 \mathrm{~mL})$. The organic layers were combined, washed with saturated $\mathrm{NaHCO}_{3}(20$ $\mathrm{mL})$, brine $(20 \mathrm{~mL})$, dried with $\mathrm{MgSO}_{4}$ and concentrated under reduced pressure. Purification by column chromatography (pentane/Et $\left.{ }_{2} \mathrm{O} 8 / 2\right)$ afforded 7 e $(53 \mathrm{mg}, 0.18 \mathrm{mmol}, 89 \%)$ as a yellow amorphous solid. Mp $55-58^{\circ} \mathrm{C} .{ }^{1} \mathrm{H} \mathrm{NMR}\left(\mathrm{CDCl}_{3}, 400 \mathrm{MHz}\right) \delta 8.11(\mathrm{br} \mathrm{s}, 1 \mathrm{H}, \mathrm{NH}), 7.79(\mathrm{~m}, 1 \mathrm{H}, \mathrm{ArH}), 7.40(\mathrm{~d}, J=2.7 \mathrm{~Hz}, 1 \mathrm{H}$, ArH), 7.36 (m, $1 \mathrm{H}, \mathrm{ArH}), 7.26$ (m, $2 \mathrm{H}, \mathrm{ArH}), 1.22$ (m, $21 \mathrm{H}, \mathrm{TIPS}) .{ }^{13} \mathrm{C}$ NMR $\left(\mathrm{CDCl}_{3}, 100 \mathrm{MHz}\right) \delta 135.1$, $128.9,128.3,123.1,120.8,120.1,111.4,100.4,99.3,92.19,18.8,11.5$. Consistent with reported data. ${ }^{2}$ 
Kinetic evaluation of the triisopropylsilyl benziodoxolone analogues

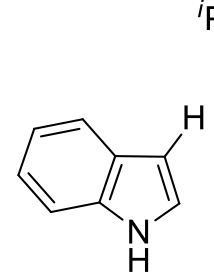

6<smiles>[R17]c1cccc2c1C(=O)OI2C#C[SiH2]C</smiles>

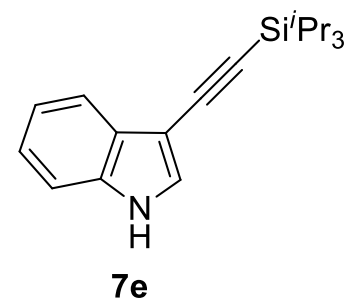

$7 e$

$\mathrm{CH}_{2} \mathrm{Cl}_{2}(3 \mathrm{~mL})$ was added to indole (6) $(11.7 \mathrm{mg}, 0.100 \mathrm{mmol}, 1.0$ equiv) in a $0.05 \mathrm{M}$ solution of undecylcyanide in $\mathrm{CH}_{2} \mathrm{Cl}_{2}$ (1 mL). Gold 2-pyridinecarboxylate dichloride (26) (2.2 mg, $0.0050 \mathrm{mmol}, 0.05$ equiv) in $\mathrm{CH}_{2} \mathrm{Cl}_{2}(1 \mathrm{~mL})$ and then the corresponding alkynyl benziodoxolone $(0.220 \mathrm{mmol}, 1.2$ equiv $)$ in $\mathrm{CH}_{2} \mathrm{Cl}_{2}{ }^{1}(1 \mathrm{~mL})$ were added under air. An aliquot of $100 \mu \mathrm{L}$ was filtered over silica gel, diluted in $\mathrm{CH}_{2} \mathrm{Cl}_{2}(1$ $\mathrm{mL}$ ) and injected in GC-FID. The yield was determined using a calibration of the product toward the internal reference undecylcyanide. Initial temperature: $50{ }^{\circ} \mathrm{C}$, Ramp: $10.0{ }^{\circ} \mathrm{C} / \mathrm{min}$ to $250{ }^{\circ} \mathrm{C}$, hold 10 min at $250{ }^{\circ} \mathrm{C}$. Retention times: indole (6): $9.39 \mathrm{~min}$, undecylcyanide: $10.60 \mathrm{~min}, 3$-((triisopropylsilyl)ethynyl)- $1 H$-indole (7e): $20.11 \mathrm{~min}$. Every reaction was carried out three times to insure reproducibility and an average of the values is used for the graphic.

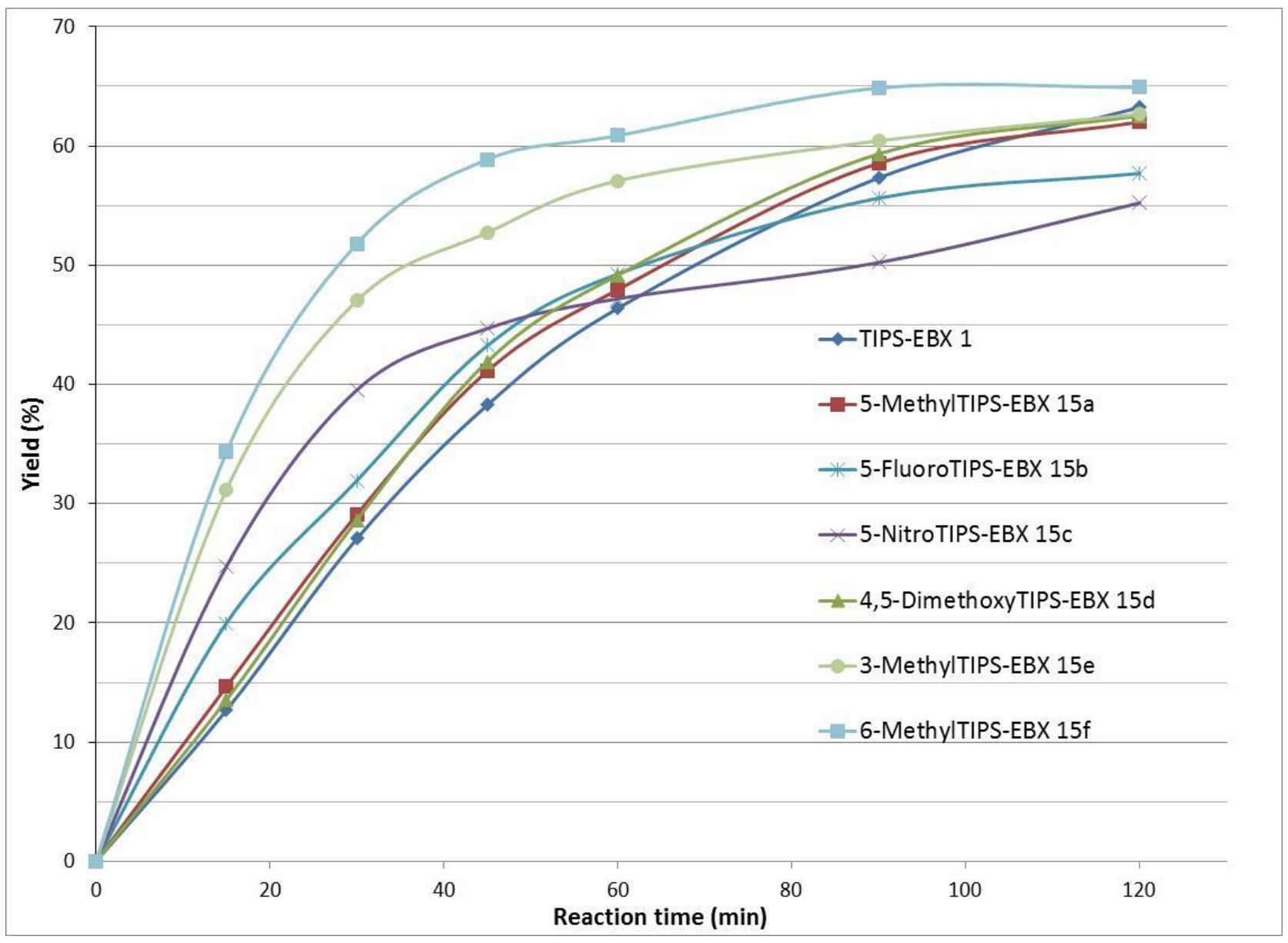




\section{Reactions with 6-methyl TIPS-EBX (15f)}

\section{3-Methyl-2-((triisopropylsilyl)ethynyl)-1H-indole (27)}

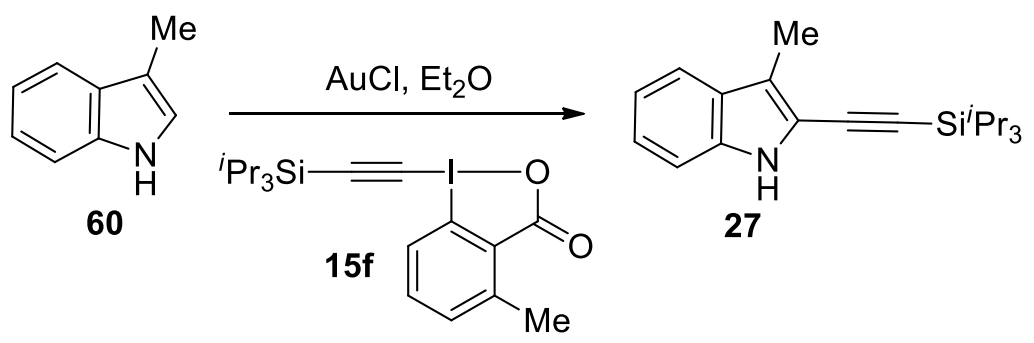

$\mathrm{AuCl}$ (2.3 mg, $0.020 \mathrm{mmol}, 0.05$ equiv) was added to a stirring solution of 6-methylTIPS-EBX (15f) (106 $\mathrm{mg}, 0.240 \mathrm{mmol}, 1.2$ equiv) and 3-methylindole (60) (26.2 mg, $0.200 \mathrm{mmol}, 1.0$ equiv) in $\mathrm{Et}_{2} \mathrm{O}(4 \mathrm{~mL})$ under air. The reaction was sealed and stirred at room temperature for $14 \mathrm{~h}$. $\mathrm{Et}_{2} \mathrm{O}(10 \mathrm{~mL})$ was added, the organic layer was washed twice with $0.1 \mathrm{M} \mathrm{NaOH}(15 \mathrm{~mL})$. The aqueous layers were combined and extracted with $\mathrm{Et}_{2} \mathrm{O}(20 \mathrm{~mL})$. The organic layers were combined, washed with saturated $\mathrm{NaHCO}_{3}(20 \mathrm{~mL})$, brine $(20 \mathrm{~mL})$, dried with $\mathrm{MgSO}_{4}$ and concentrated under reduced pressure. Purification by column chromatography (pentane/ $\mathrm{CH}_{2} \mathrm{Cl}_{2}$ 95/5) afforded 27 (52 $\mathrm{mg}, 0.17 \mathrm{mmol}, 84 \%$ ) as an amorphous orange solid. $\mathrm{R}_{f} 0.1$ (pentane/ $\mathrm{CH}_{2} \mathrm{Cl}_{2}$ 95/5). Mp 75-77 ${ }^{\circ} \mathrm{C} .{ }^{1} \mathrm{H} \mathrm{NMR}\left(\mathrm{CDCl}_{3}, 400 \mathrm{MHz}\right) \delta 7.98$ (br s, $\left.1 \mathrm{H}, \mathrm{NH}\right), 7.59$ $(\mathrm{d}, J=7.9 \mathrm{~Hz}, 1 \mathrm{H}, \operatorname{ArH}), 7.28(\mathrm{~m}, 2 \mathrm{H}, \operatorname{ArH}), 7.17(\mathrm{~m}, 1 \mathrm{H}, \mathrm{ArH}), 2.47(\mathrm{~d}, J=0.5 \mathrm{~Hz}, 3 \mathrm{H}, \mathrm{Me}), 1.23(\mathrm{~m}$, $21 \mathrm{H}$, TIPS). ${ }^{13} \mathrm{C} \mathrm{NMR}\left(\mathrm{CDCl}_{3}, 100 \mathrm{MHz}\right) \delta 135.6,127.8,123.7,119.8,119.3,118.9,117.0,110.7,98.5$, 97.4, 18.8, 11.4, 9.6. Consistent with reported data. 2

\section{1-(5-((Triisopropylsilyl)ethynyl)-1H-pyrrol-3-yl)ethanone (28)}<smiles>CC(=O)c1cc[nH]c1</smiles><smiles>COCCOI(C#C[Hg]Br)c1cccc(C)c1C(=O)OCC(C)C</smiles><smiles>CC(=O)c1c[nH]c(C#C[SiH2]C(=O)[OH2+])c1</smiles>

$\mathrm{AuCl}$ (2.3 mg, $0.020 \mathrm{mmol}, 0.05$ equiv) was added to a stirring solution of 6-methylTIPS-EBX (21f) (106 $\mathrm{mg}, 0.240 \mathrm{mmol}, 1.2$ equiv), 3-acetylpyrrole 61 (22.0 mg, $0.200 \mathrm{mmol}, 1.0$ equiv) in $\mathrm{Et}_{2} \mathrm{O}^{1}$ (4 mL) under air. The reaction was sealed and stirred at room temperature for $15 \mathrm{~h}$. $\mathrm{Et}_{2} \mathrm{O}(10 \mathrm{~mL})$ was added and the organic layer was washed twice with $0.1 \mathrm{M} \mathrm{NaOH}(15 \mathrm{~mL})$. The aqueous layers were combined and extracted with $\mathrm{Et}_{2} \mathrm{O}(20 \mathrm{~mL})$. The organic layers were combined, washed with saturated $\mathrm{NaHCO}_{3}(20 \mathrm{~mL})$, brine $(20 \mathrm{~mL})$, dried with $\mathrm{MgSO}_{4}$ and concentrated under reduced pressure. Purification by column chromatography (pentane $/ \mathrm{Et}_{2} \mathrm{O} 7 / 3$ ) afforded 28 (40 mg, $0.14 \mathrm{mmol}, 69 \%$ ) as a yellow solid. $\mathrm{R}_{f} 0.3\left(\mathrm{PET} / \mathrm{Et}_{2} \mathrm{O} 7 / 3\right.$ ). Mp 120$122{ }^{\circ} \mathrm{C} .{ }^{1} \mathrm{H}$ NMR $\left(\mathrm{CDCl}_{3}, 400 \mathrm{MHz}\right) \delta 9.52$ (br s, $\left.1 \mathrm{H}, \mathrm{NH}\right), 7.37$ (dd, $\left.1 \mathrm{H}, J=2.6,1.2 \mathrm{~Hz}, \mathrm{ArH}\right), 6.87(\mathrm{~m}$, $1 \mathrm{H}, \mathrm{ArH}), 2.44$ (s, $3 \mathrm{H}, \mathrm{Ac}), 1.10$ (m, $21 \mathrm{H}, \mathrm{TIPS}) .{ }^{13} \mathrm{C} \mathrm{NMR}\left(\mathrm{CDCl}_{3}, 100 \mathrm{MHz}\right) \delta 193.7,126.2,124.1$, 115.3, 115.1, 97.4, 93.4, 27.3, 18.6, 11.3. Consistent with reported data. 2

\section{Stoichiometric experiments}

Stoichiometric mixture of gold 2-pyridinecarboxylate dichloride and TIPS-EBX (1): 


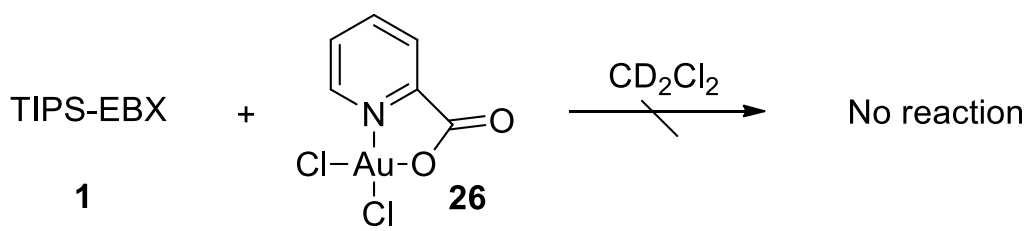

Gold 2-pyridinecarboxylate dichloride (26) $(7.8 \mathrm{mg}, 0.020 \mathrm{mmol}, 1$ equiv) and TIPS-EBX (1) (8.6 mg, 0.020 mmol, 1 equiv) were dissolved in $\mathrm{CD}_{2} \mathrm{Cl}_{2}(2 \mathrm{~mL}) .{ }^{1} \mathrm{H}$ NMR indicated no change.

Stoichiometric mixture of gold 2-pyridinecarboxylate dichloride and indole:

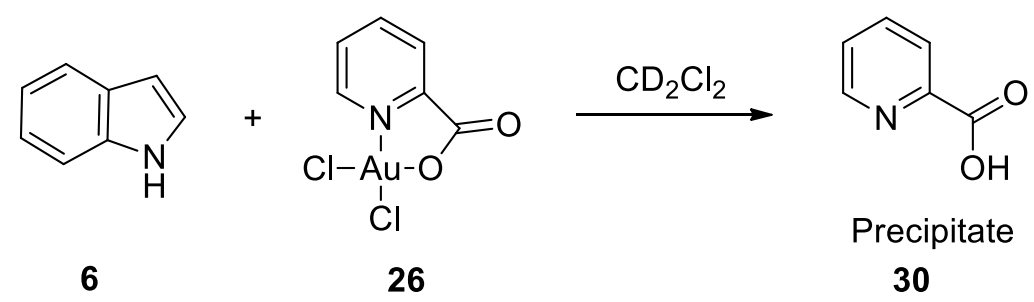

Indole (6) (4.7 $\mathrm{mg}, 0.040 \mathrm{mmol}, 2$ equiv) in $\mathrm{CD}_{2} \mathrm{Cl}_{2}(2 \mathrm{~mL})$ was added to gold 2-pyridinecarboxylate dichloride (26) (7.8 $\mathrm{mg}, 0.020 \mathrm{mmol}, 1$ equiv). After $5 \mathrm{~min}$ a brown precipitate appeared (after filtration the solid can be identified as picolinic acid (30) by comparison to the spectra of commercial picolinic acid). When TIPS-EBX (1) (17 mg, $0.040 \mathrm{mmol}, 2$ equiv) is added to the suspension, full conversion is observed by ${ }^{1} \mathrm{H}$ NMR after $1 \mathrm{~h}$.

\section{Spectra of New Compounds}


solvent: <CDCI3 >

Frequency. $400.13 \mathrm{MHz}$
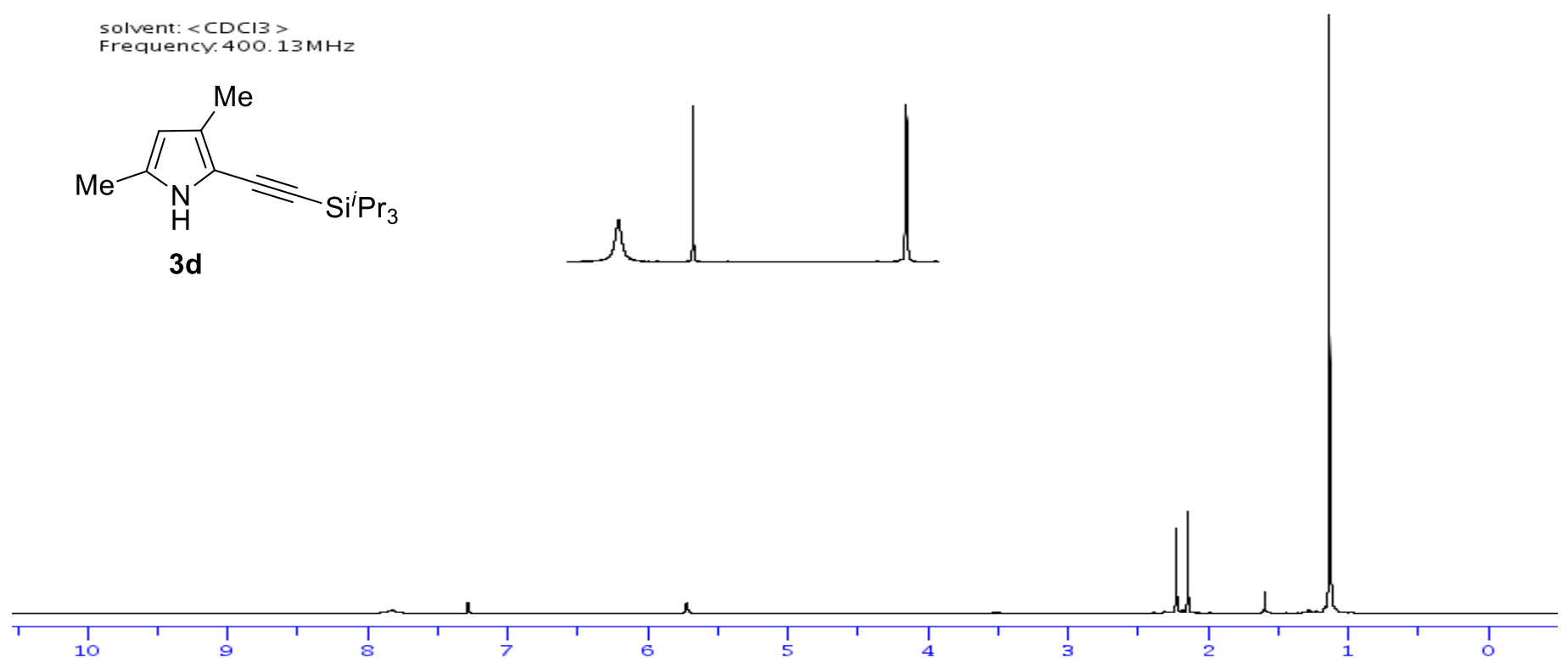

solvent: $<C D C I 3>$
Frequency. $100.612769 \mathrm{MHz}$
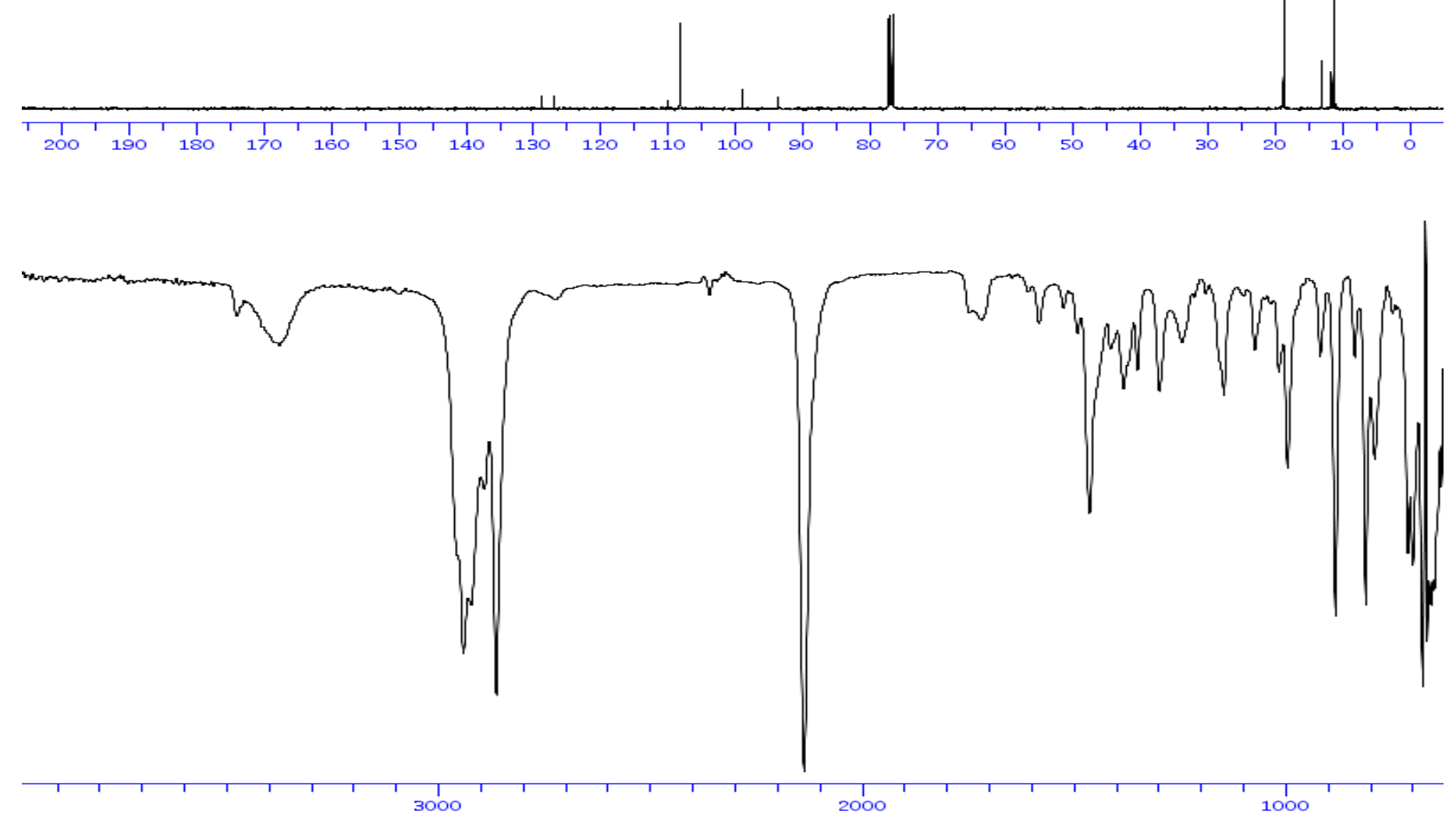
solvent: $\langle\mathrm{CDCI} 3\rangle$

(3)

${ }^{i} \mathrm{Pr}_{3} \mathrm{~S}$

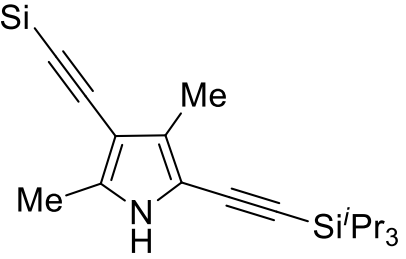

31

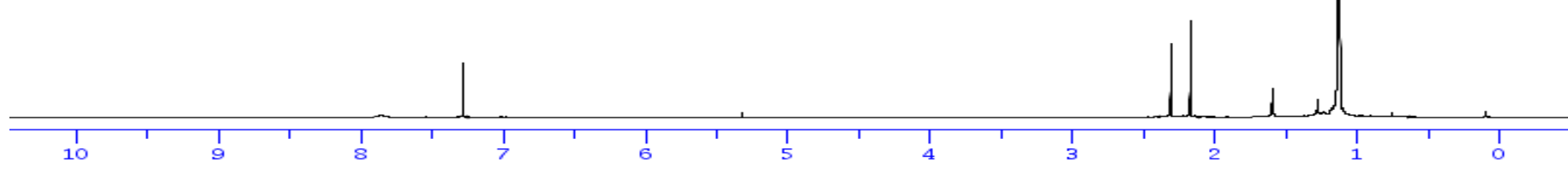

solvent: $\langle\mathrm{CDCI} 3\rangle$

Frequency. 100.612769MHz
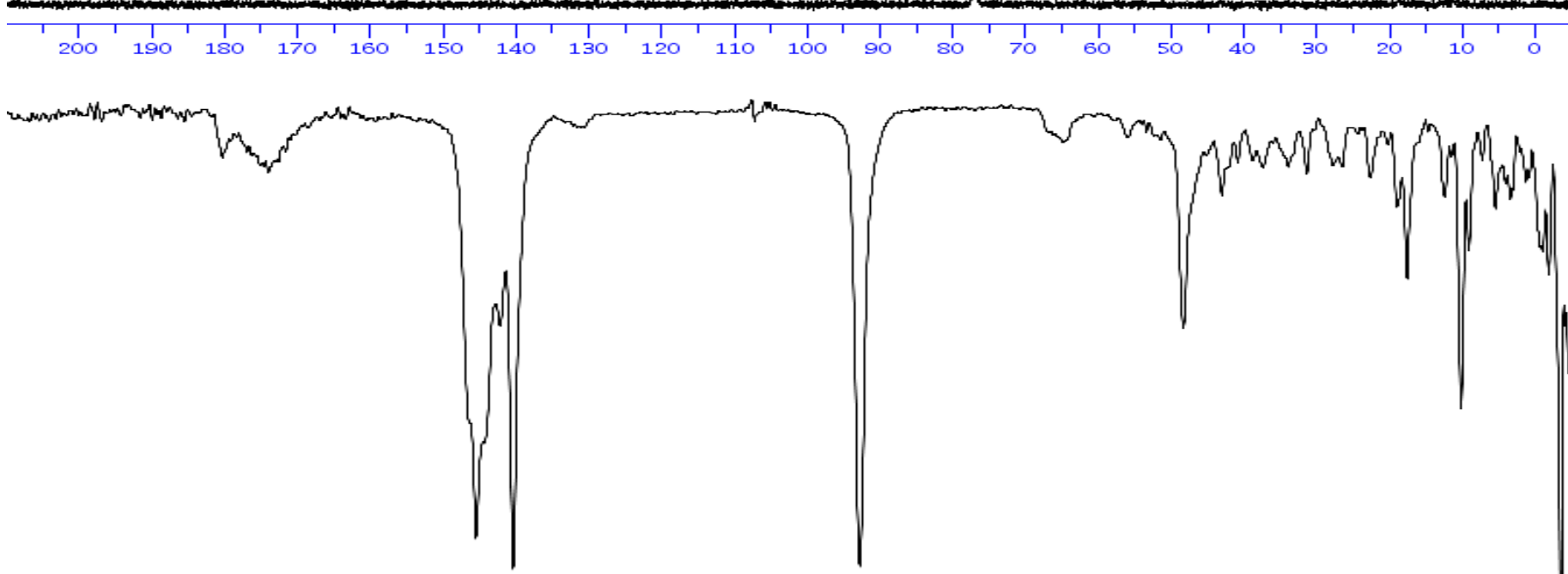
solvent: $\angle \mathrm{CDCI} 3$

Frequency. $400.13 \mathrm{MHz}$
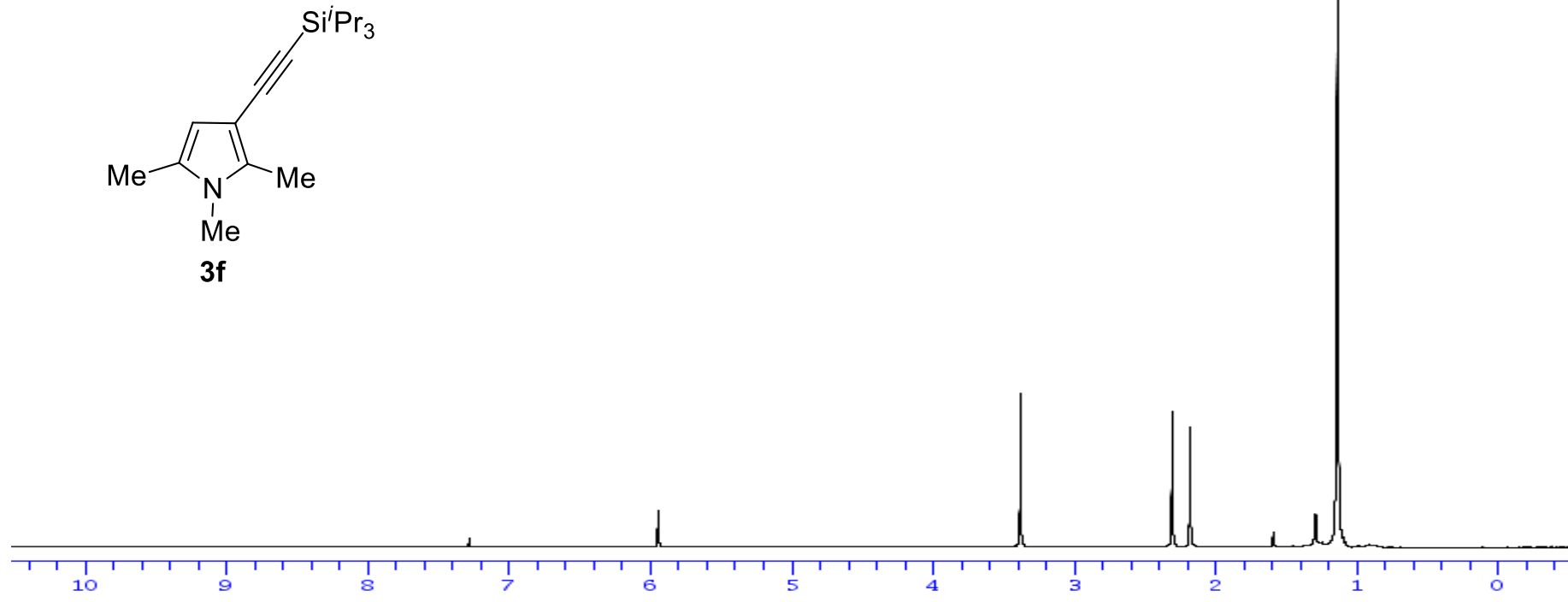

Solvent: $<C D C I 3>$
Frequency. $100.612769 \mathrm{MHz}$
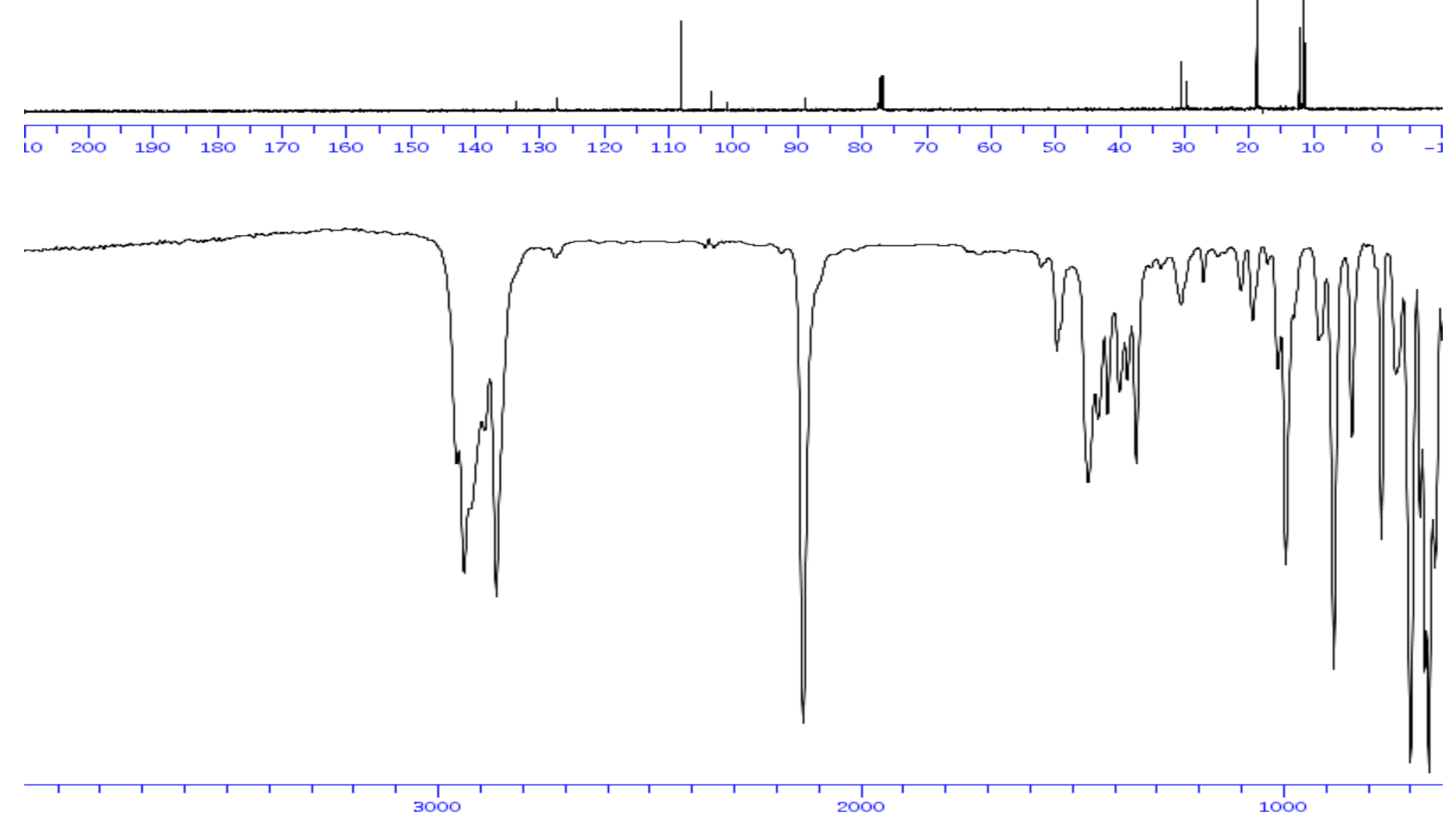
solvent: $<\mathrm{CDCI} 3>$

. $400.13 \mathrm{MHz}$

${ }^{t} \mathrm{BuMe}_{2} \mathrm{Si}=$

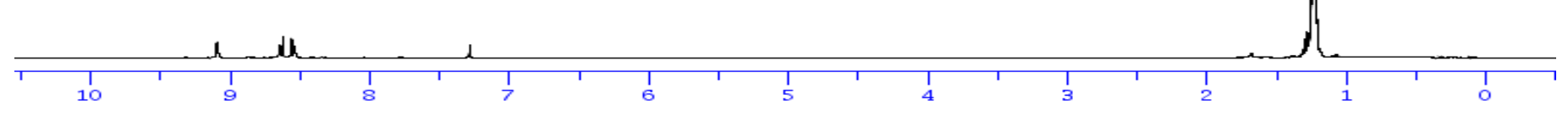

solvent: $\langle\mathrm{CDCl}\rangle$

Frequency. $100.612769 \mathrm{MHz}$
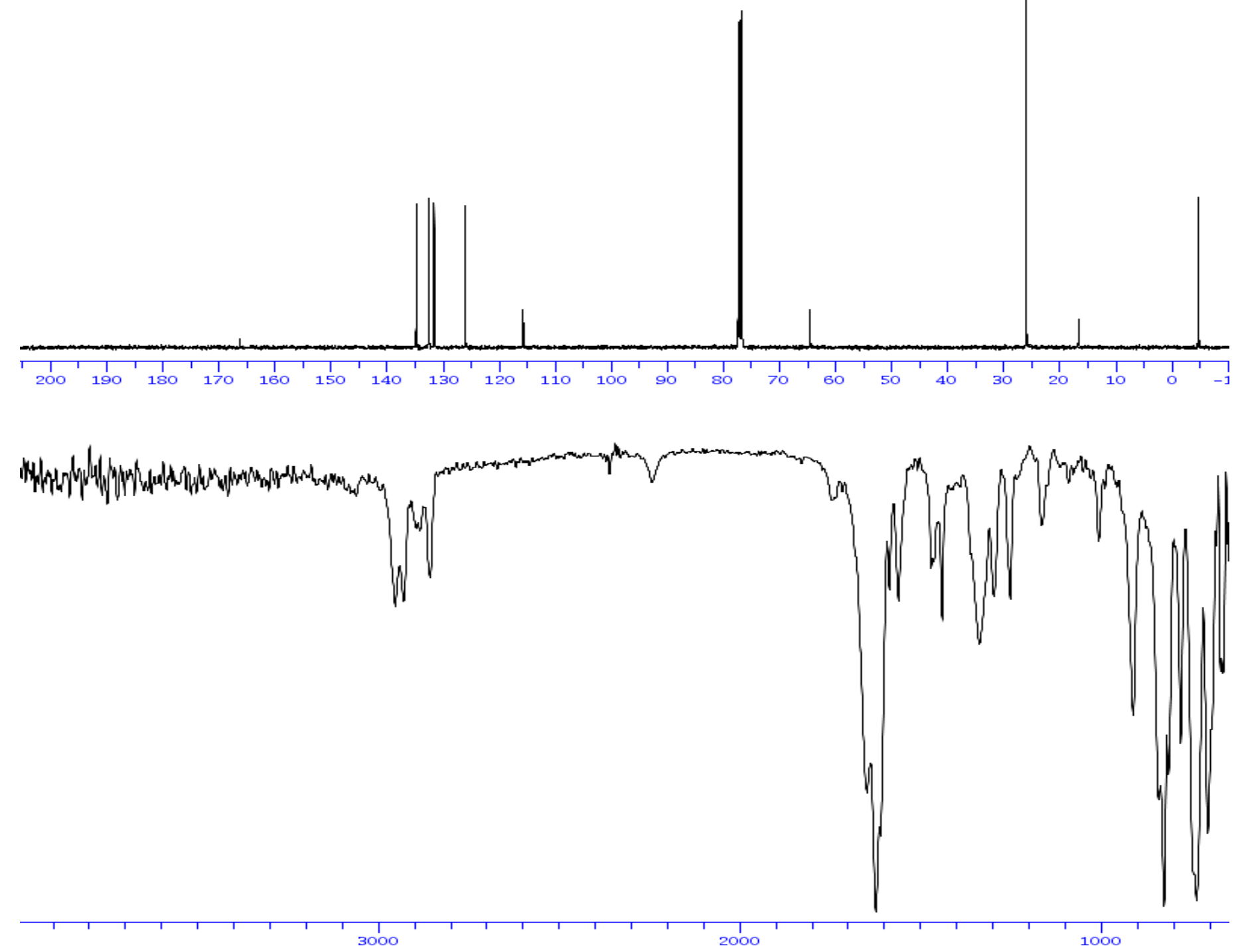
solvent: $<C D C I 3>$

Frequency.400.13MHz

${ }^{t} \mathrm{BuPh}_{2} \mathrm{Si}$

$5 d$<smiles>C#CI1OC(=O)c2ccccc21</smiles>

$M d x$

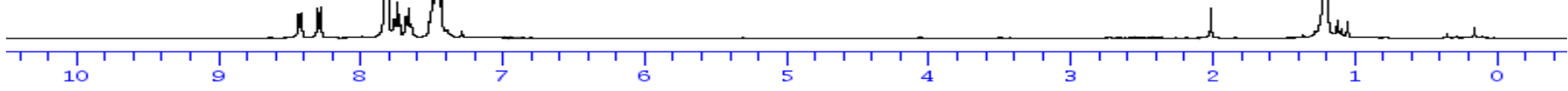

Solvent: <CDCI3 >

Frequency. $100.612769 \mathrm{MHz}$
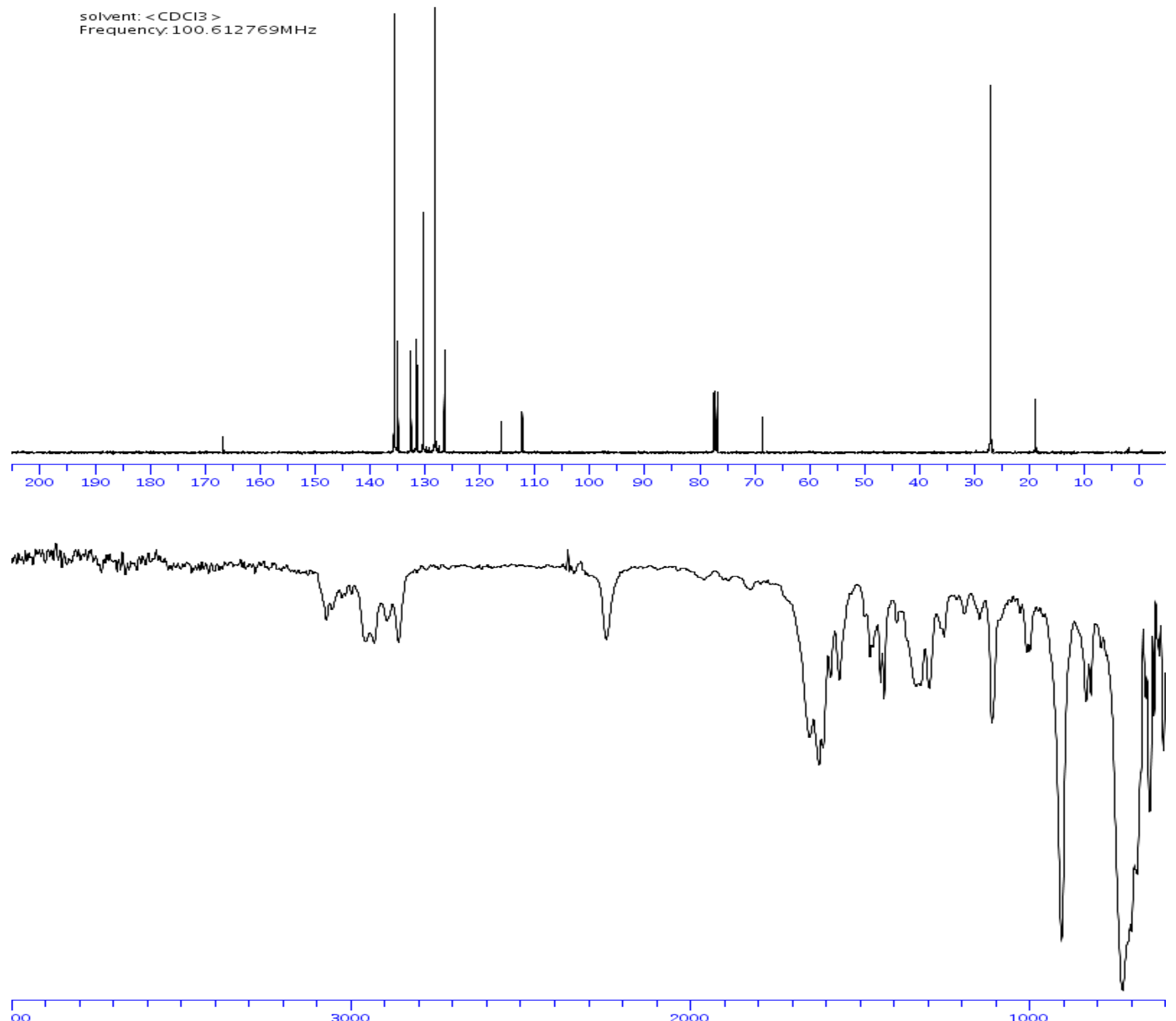
solvent: $<\mathrm{CDCI} 3$

Frequency. $400.13 \mathrm{MHz}$
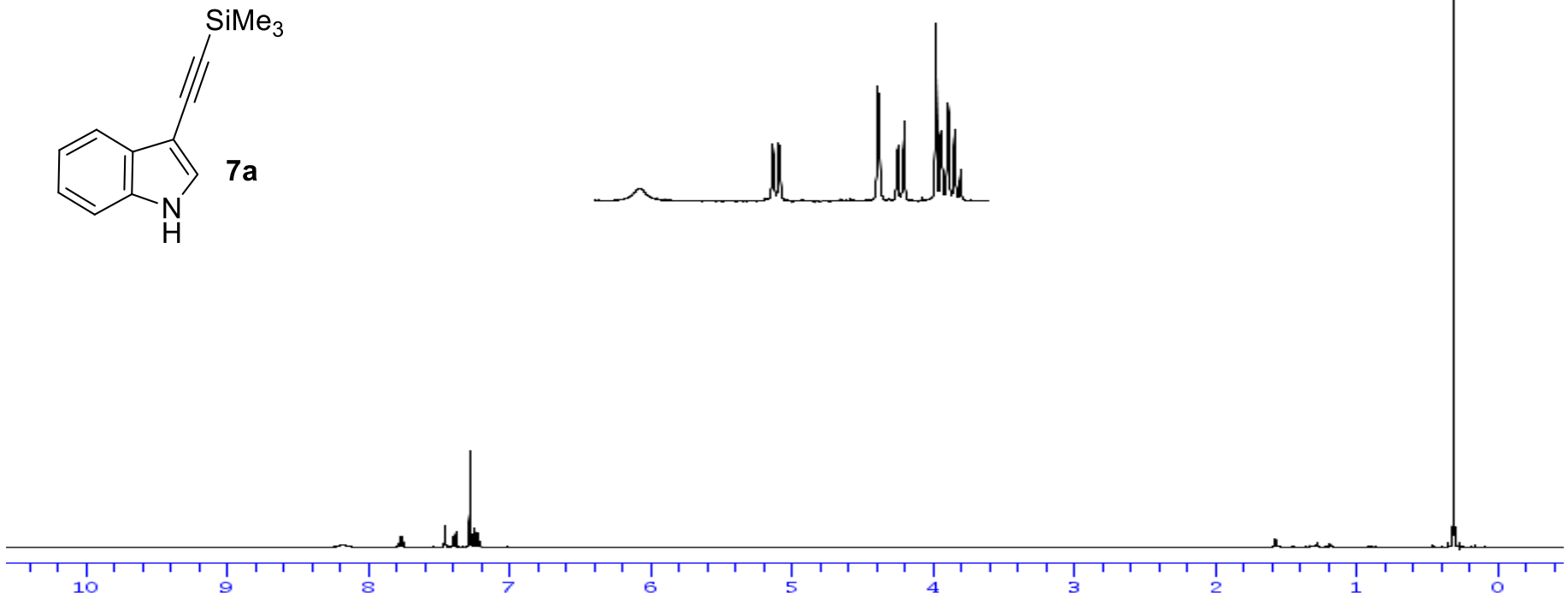

solvent: $\langle\mathrm{CDCI}\rangle\rangle$

Frequency. $100.612769 \mathrm{MHz}$
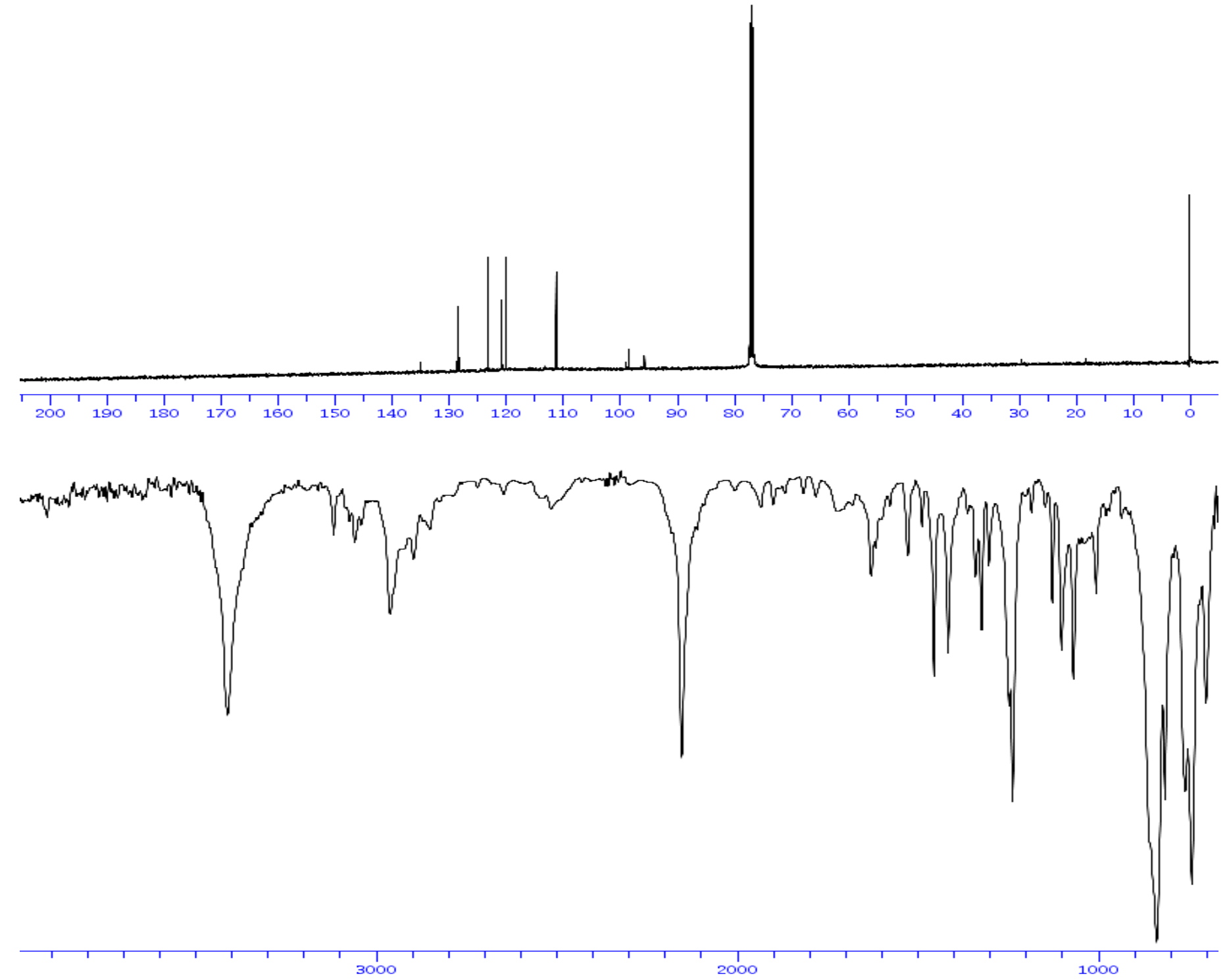
Solvent: $<\mathrm{CDCI} 3>$
Frequency. $400.13 \mathrm{MHz}$

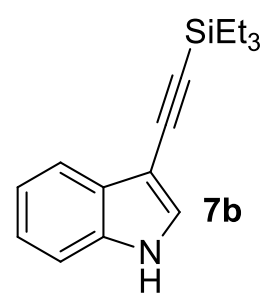

$a$ d

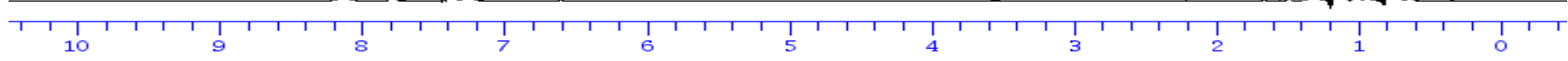

solvent: $<\mathrm{CDCI}$ >

requency. $100.612769 \mathrm{MHz}$
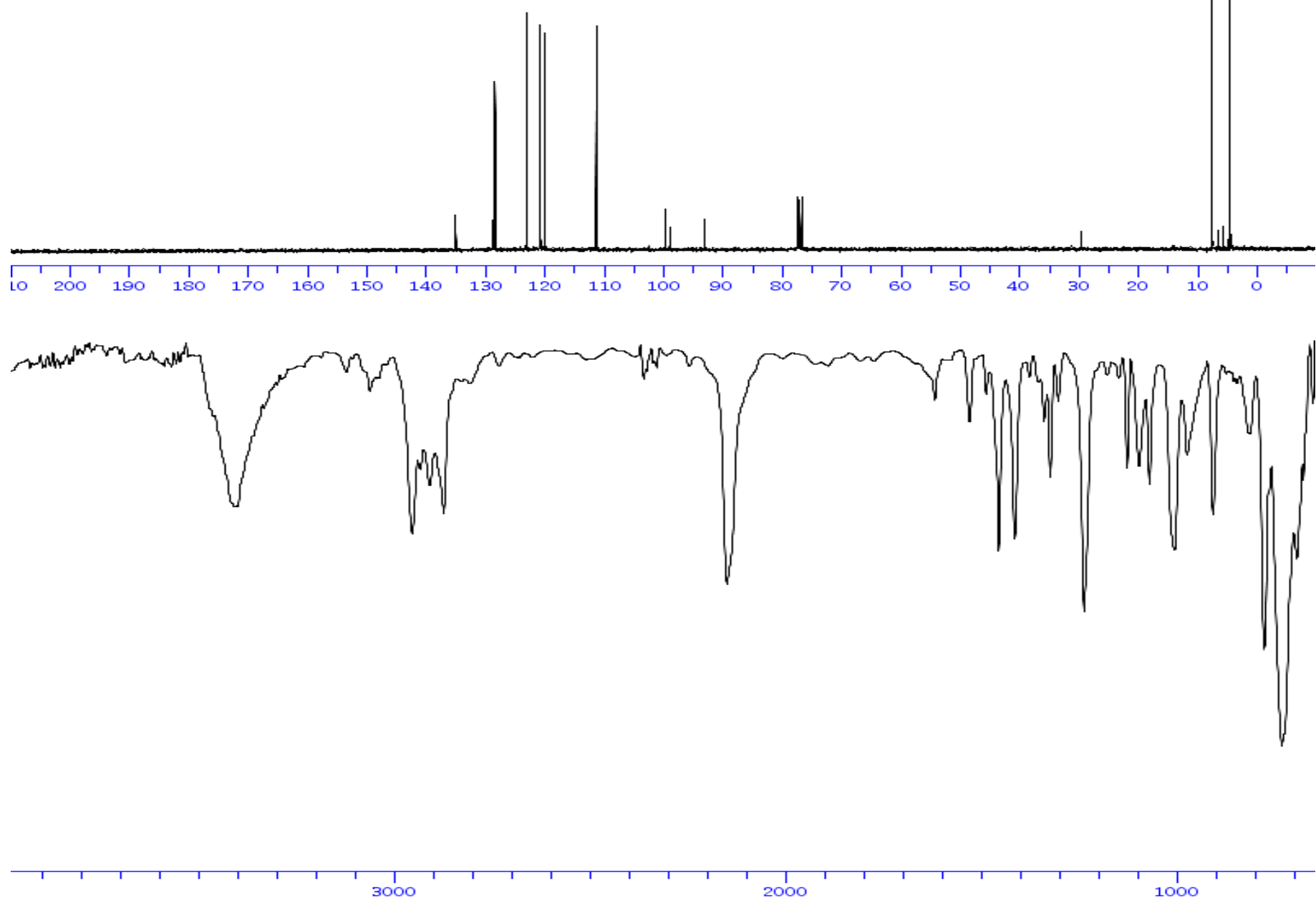
solvent: $<\mathrm{CDCI} 3$

Frequency:400.13MHz
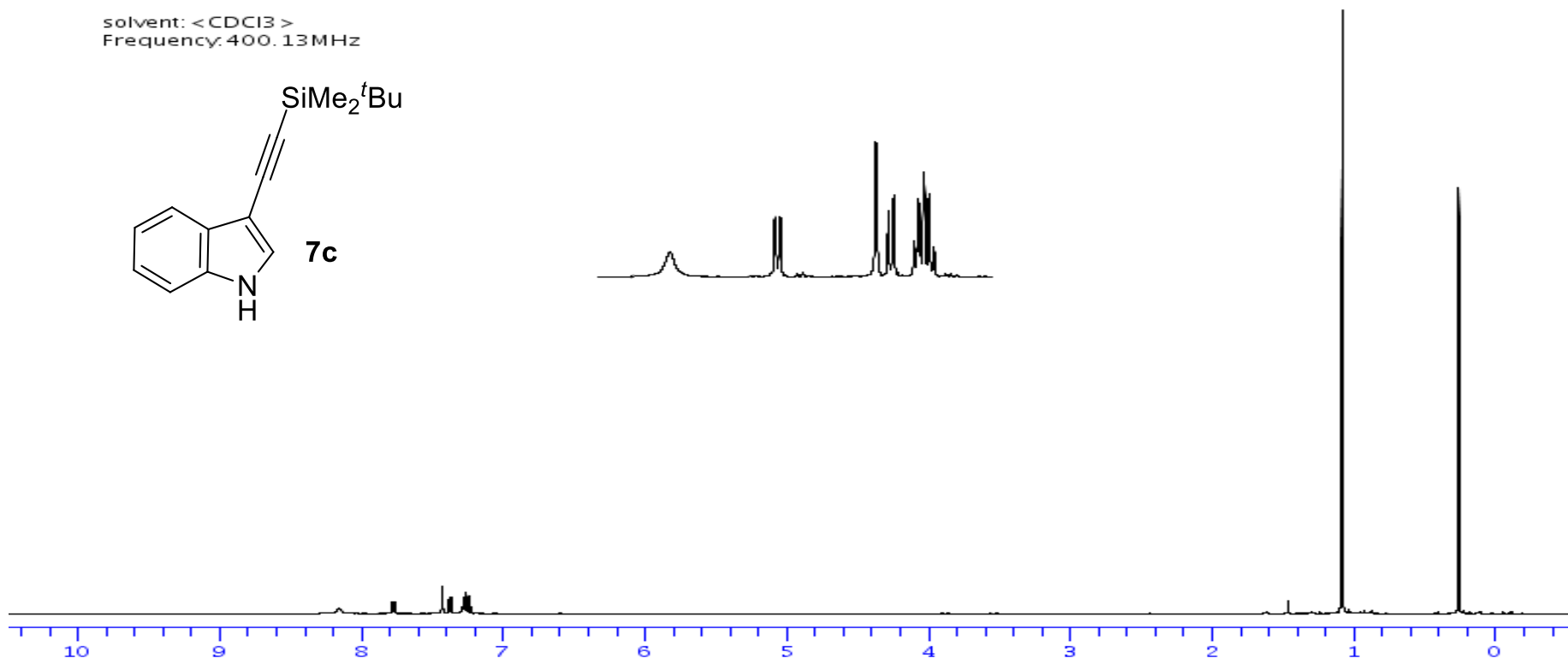

solvent: <CDCI3 $>$
Frequency. $100.612769 \mathrm{MHz}$
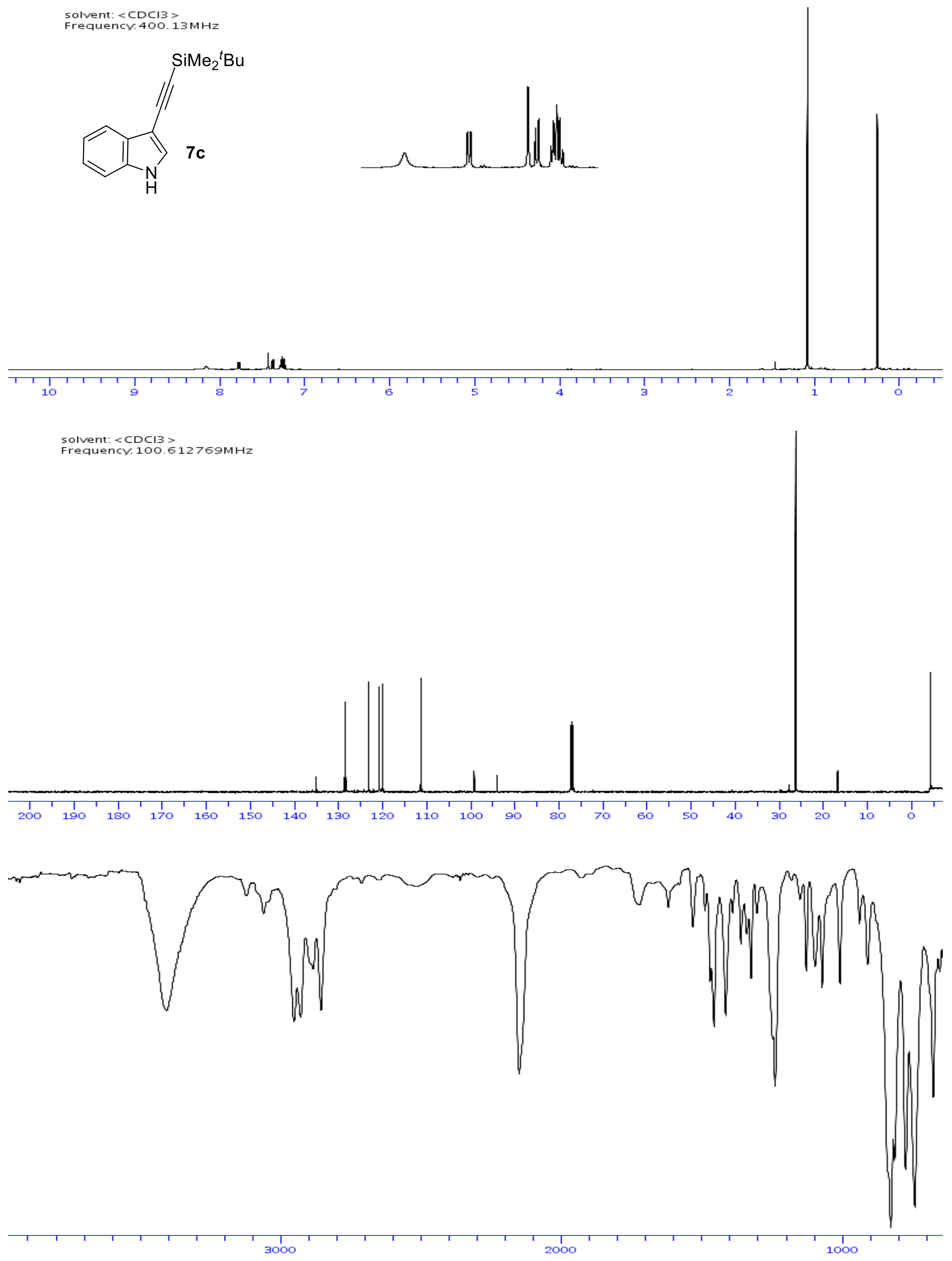
solvent: $<\mathrm{CDCI} 3>$
Frequency. $400.13 \mathrm{MHz}$
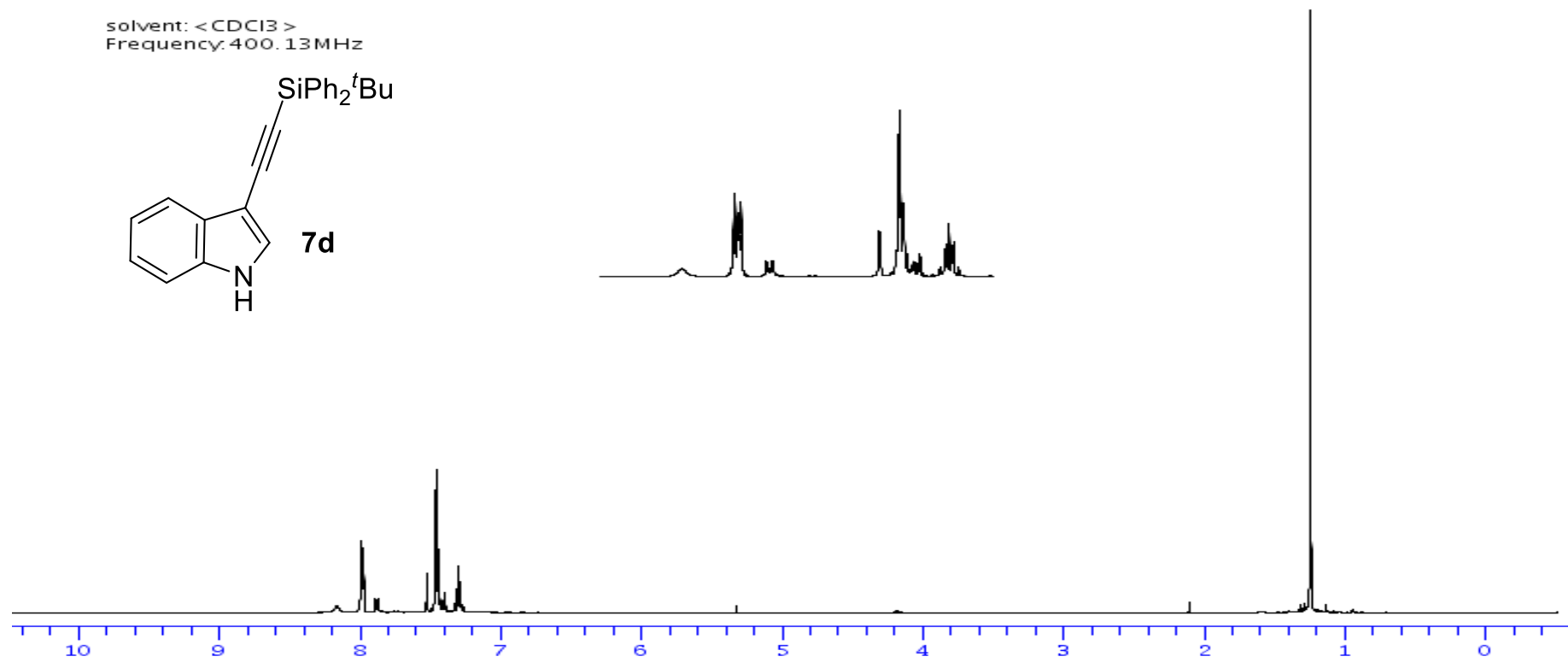

solvent: $\langle\mathrm{CDCl} 3\rangle$

Frequency. $100.612769 \mathrm{MHz}$

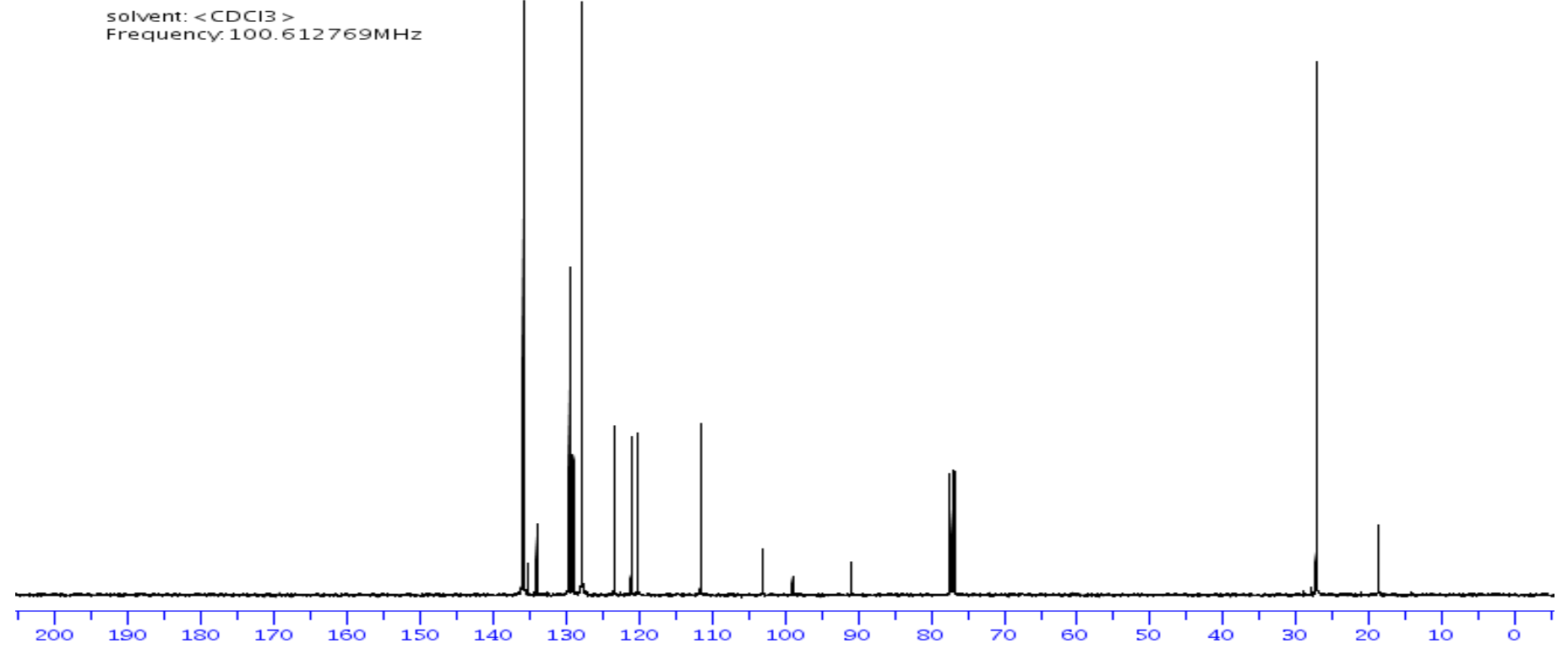

$\left\{\int_{3}\right.$

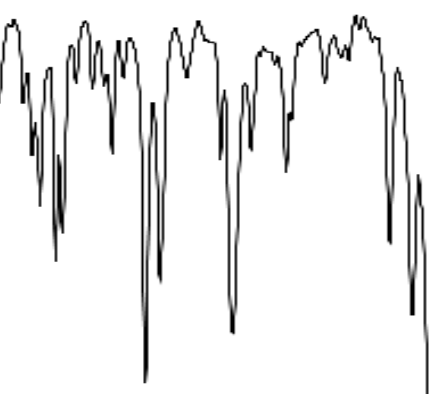


solvent: $\langle\mathrm{CDCI} 3\rangle$

Frequency $400.13 \mathrm{MHz}$

$\overbrace{\mathrm{Sex}_{\mathbf{9 b}}}=\mathrm{SiEt}_{3}$

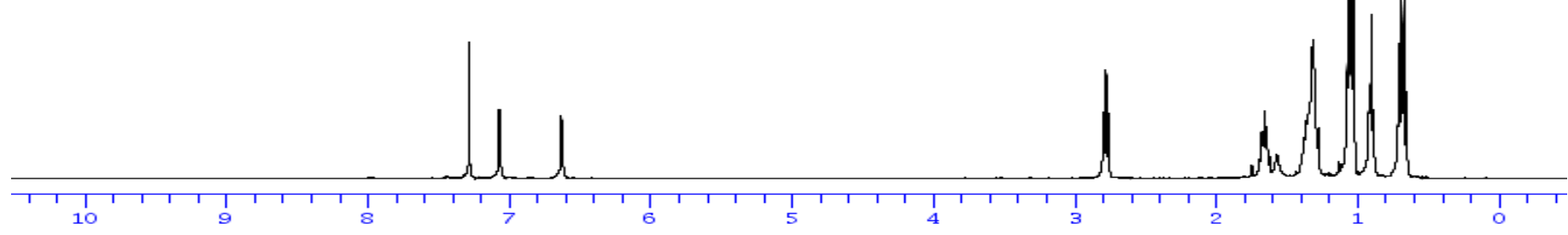

solvent: $<\mathrm{CDCI}>$

Frequency. 100.612769MHz
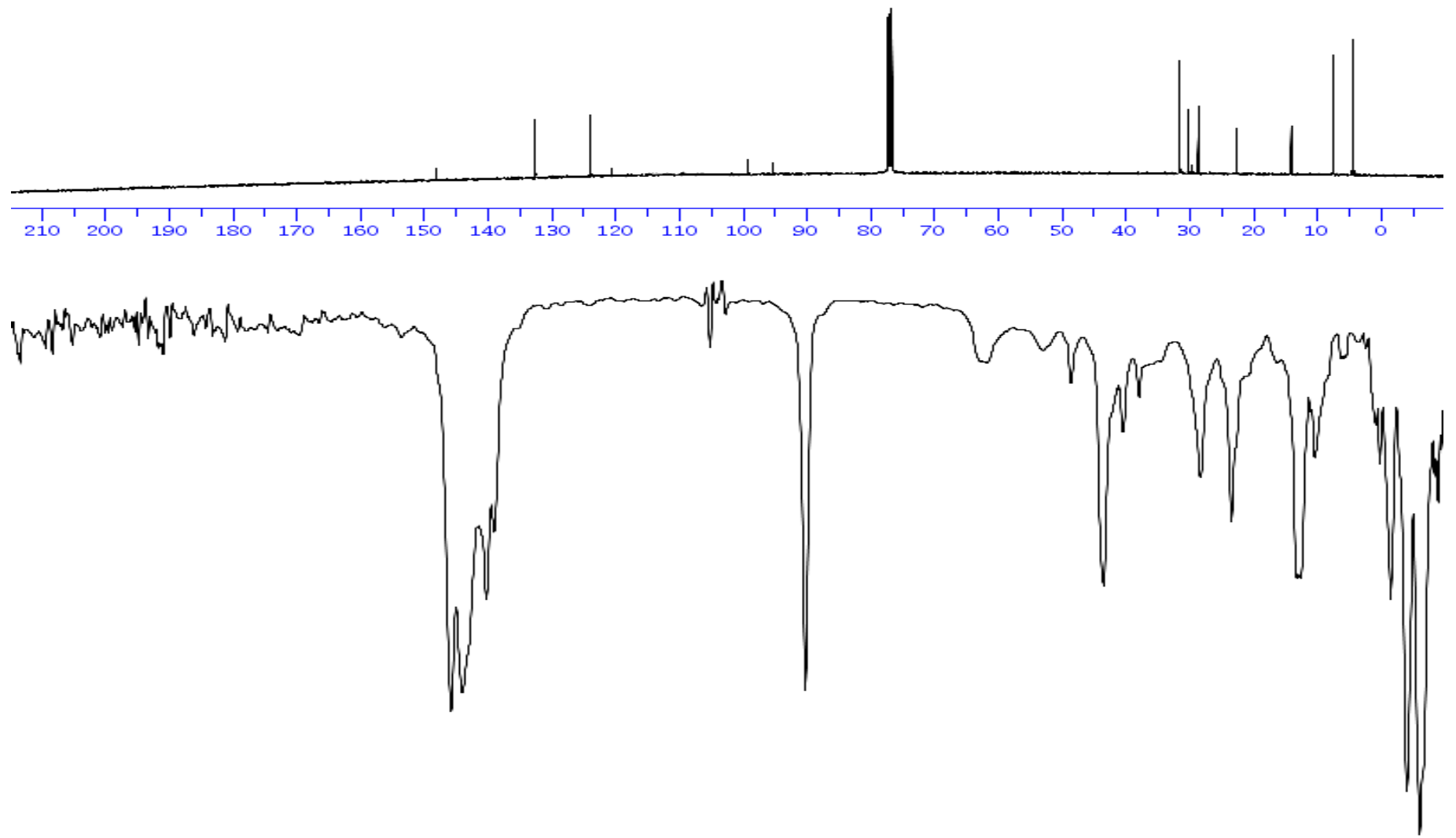
solvent: $<\mathrm{CDCI} 3$

Frequencr $400.13 \mathrm{MHz}$

$\overbrace{S \mathrm{~S}}=\mathrm{Si}_{9 \mathrm{c}}^{\mathrm{t} B u M \mathrm{M}_{2}}$$$
\text { ( }
$$
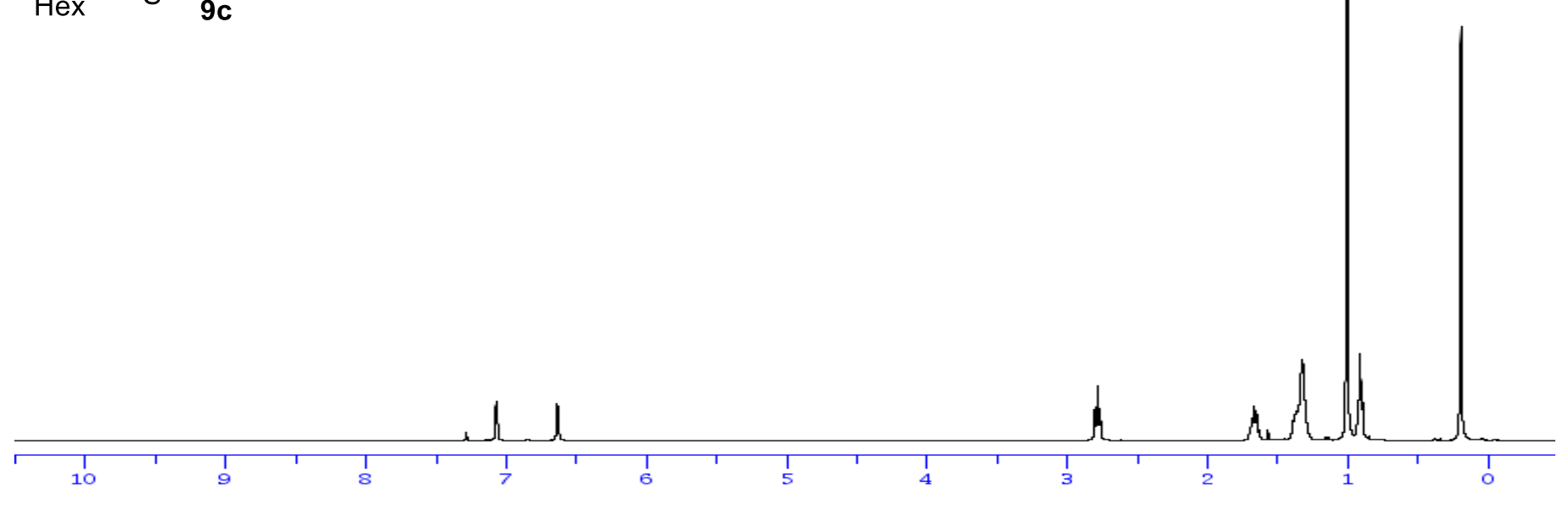

solvent: $<C D C I 3>$

Frequency. $100.612769 \mathrm{MHz}$
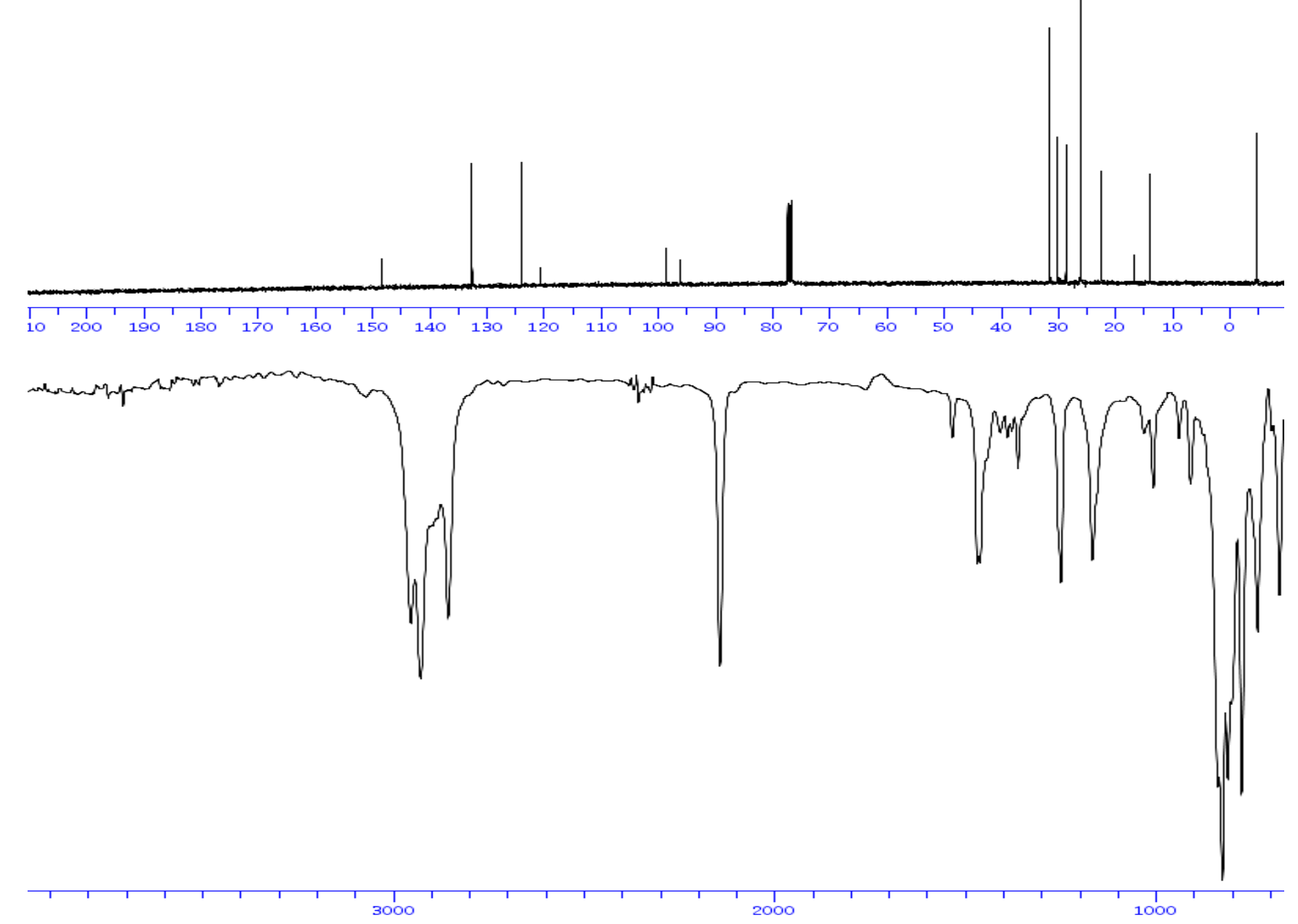


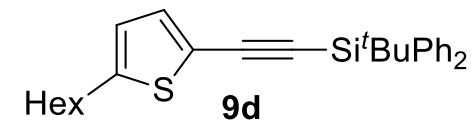

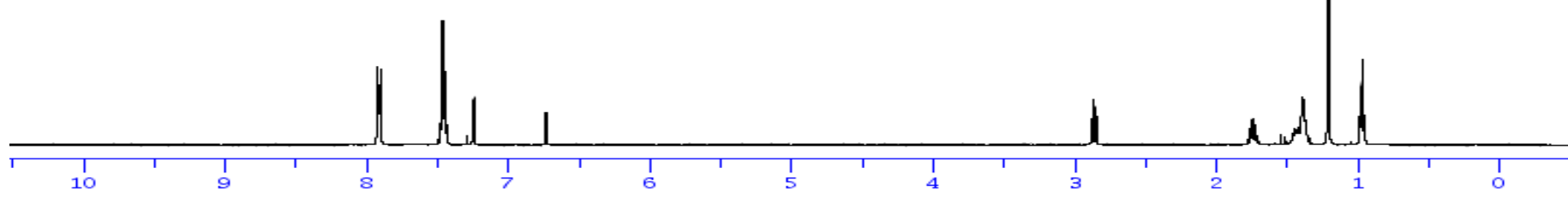

solvent: $\langle\mathrm{CDCI} 3\rangle$

Frequency. $100.612769 \mathrm{MHz}$
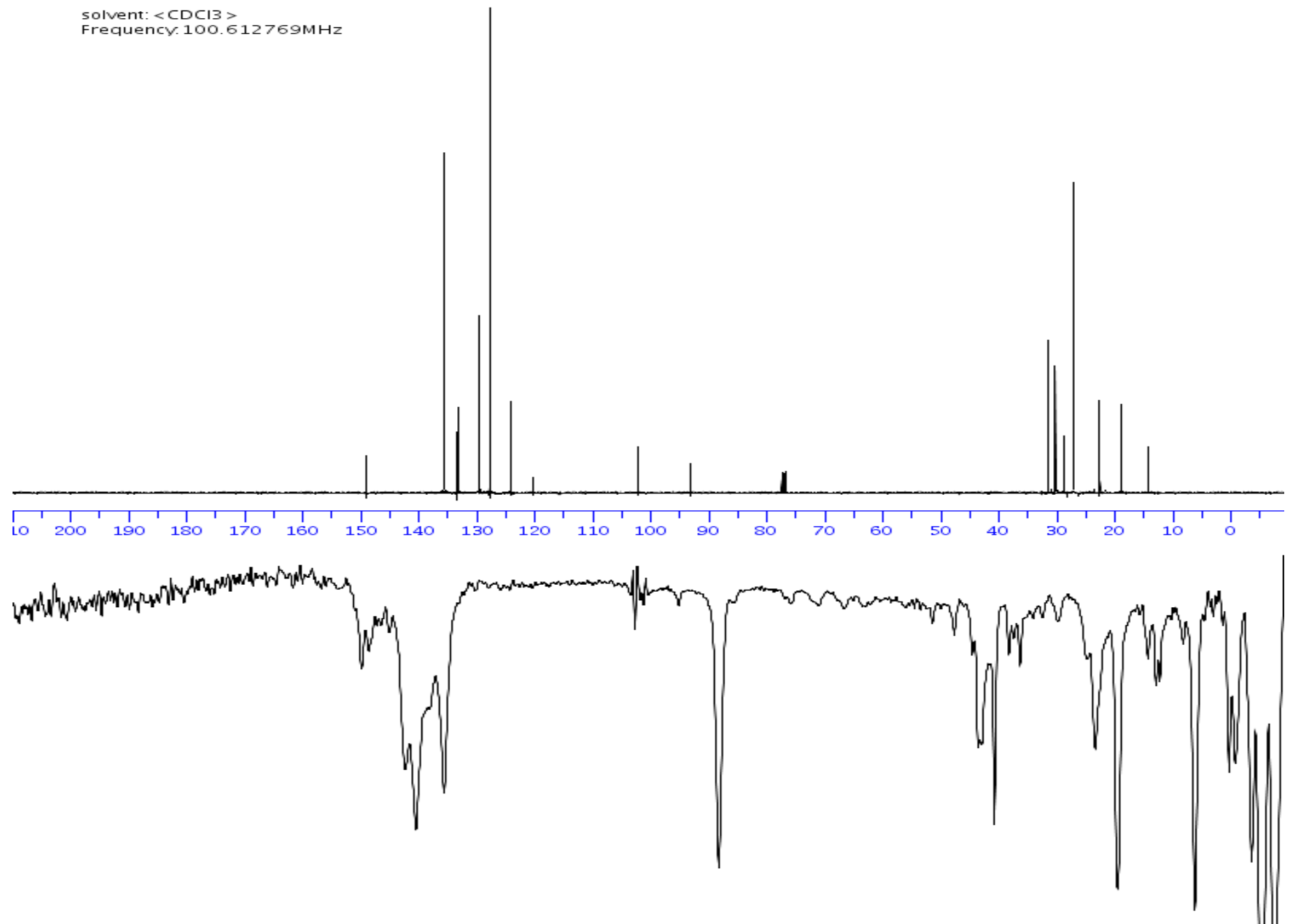

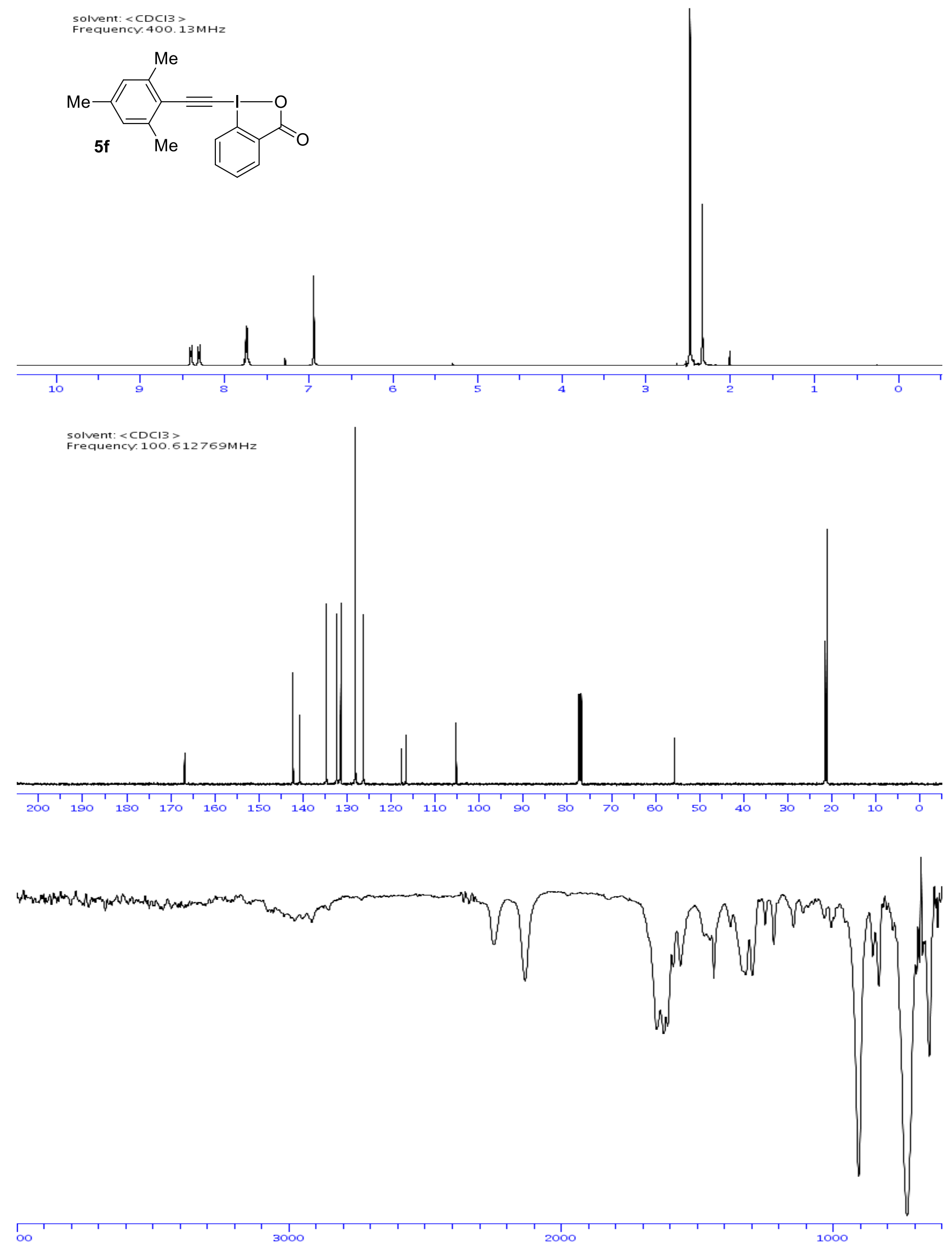
Solvent: $<C D C I 3>$
Frequency. $400.13 \mathrm{MHz}$

$\mathrm{O}_{2} \mathrm{~N}-$

$5 g$<smiles>C#CI1OC(=O)c2ccccc21</smiles>

dell

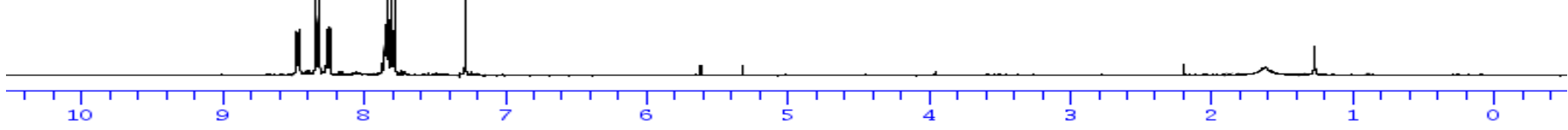

solvent: $\langle\mathrm{CDCI} 3\rangle$

Frequency. $100.612769 \mathrm{MHz}$

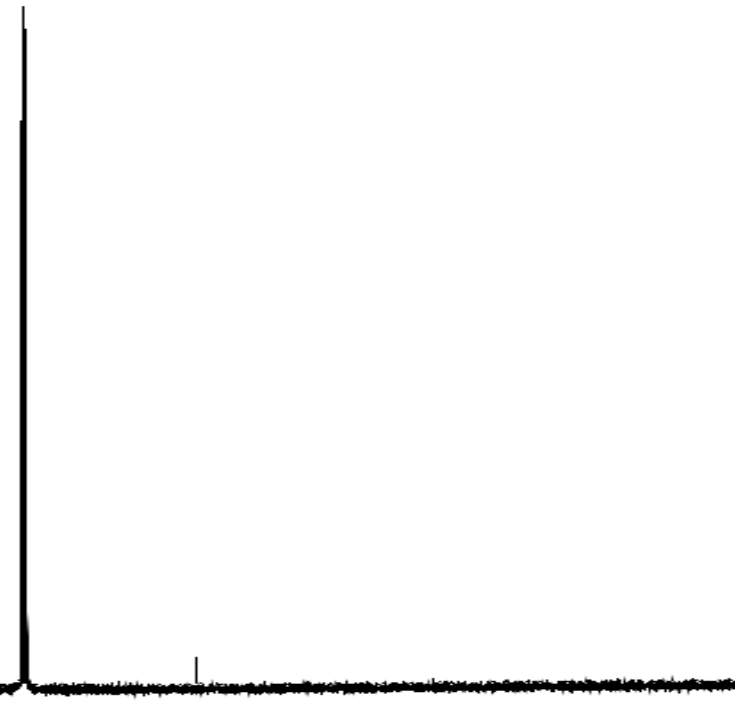

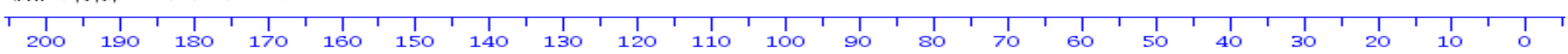
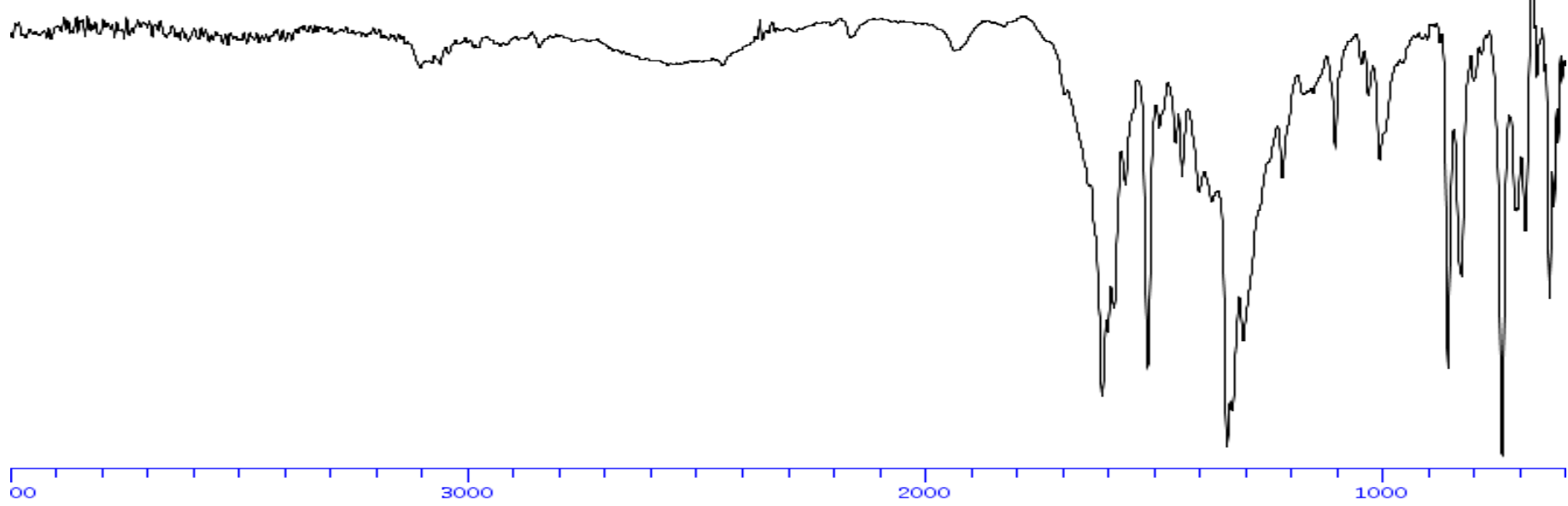


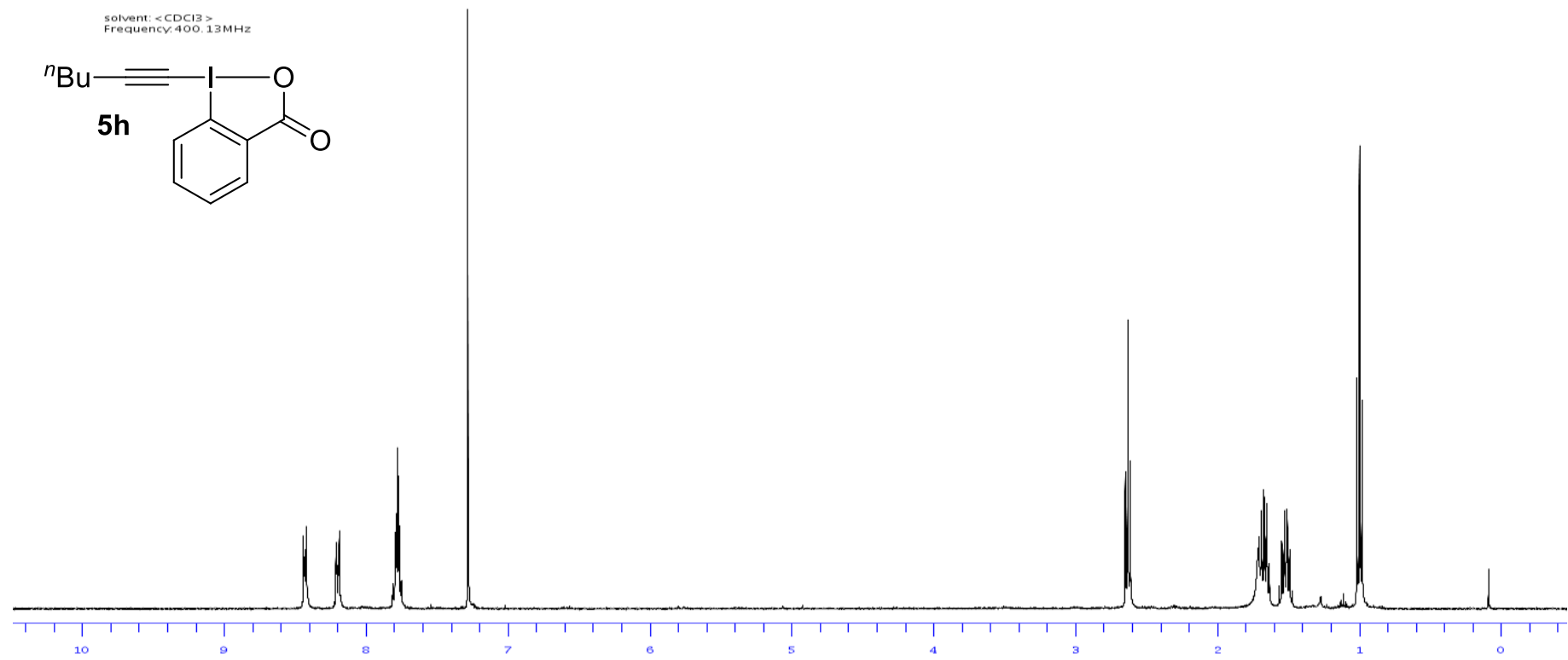

solvent: $\langle\mathrm{CDCI} 3\rangle$

Frequency. $100.612769 \mathrm{MHz}$

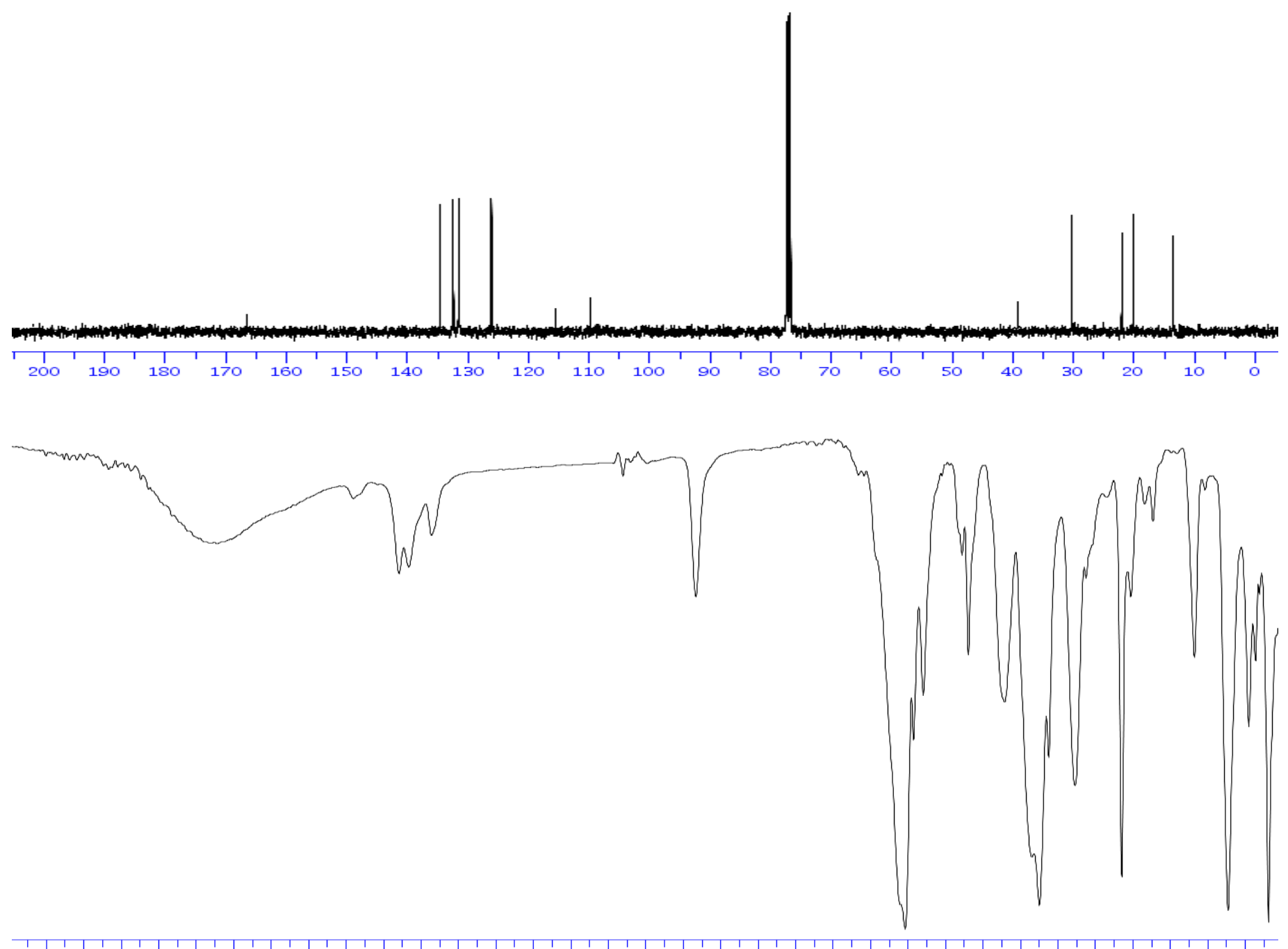


solvent: $<C D C I 3>$

Frequency $400.13 \mathrm{MHz}$
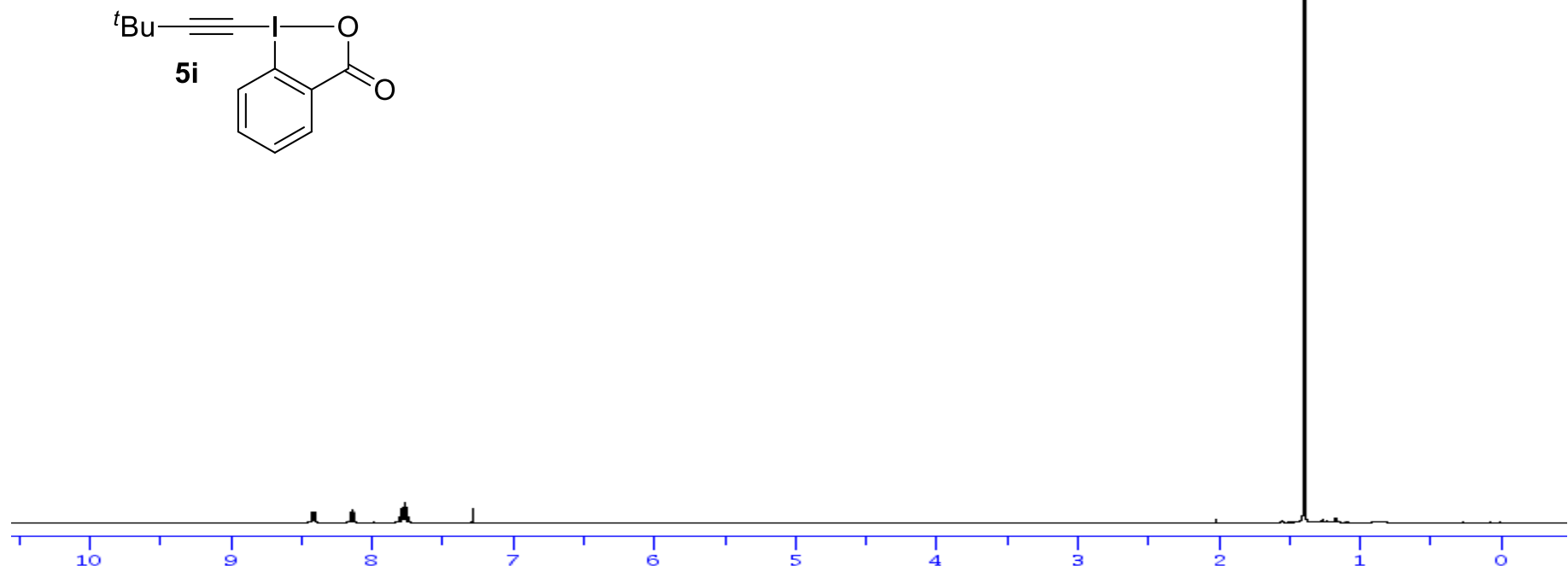

solvent: $\angle C D C I 3>$
Frequency. $100.612769 \mathrm{MHz}$
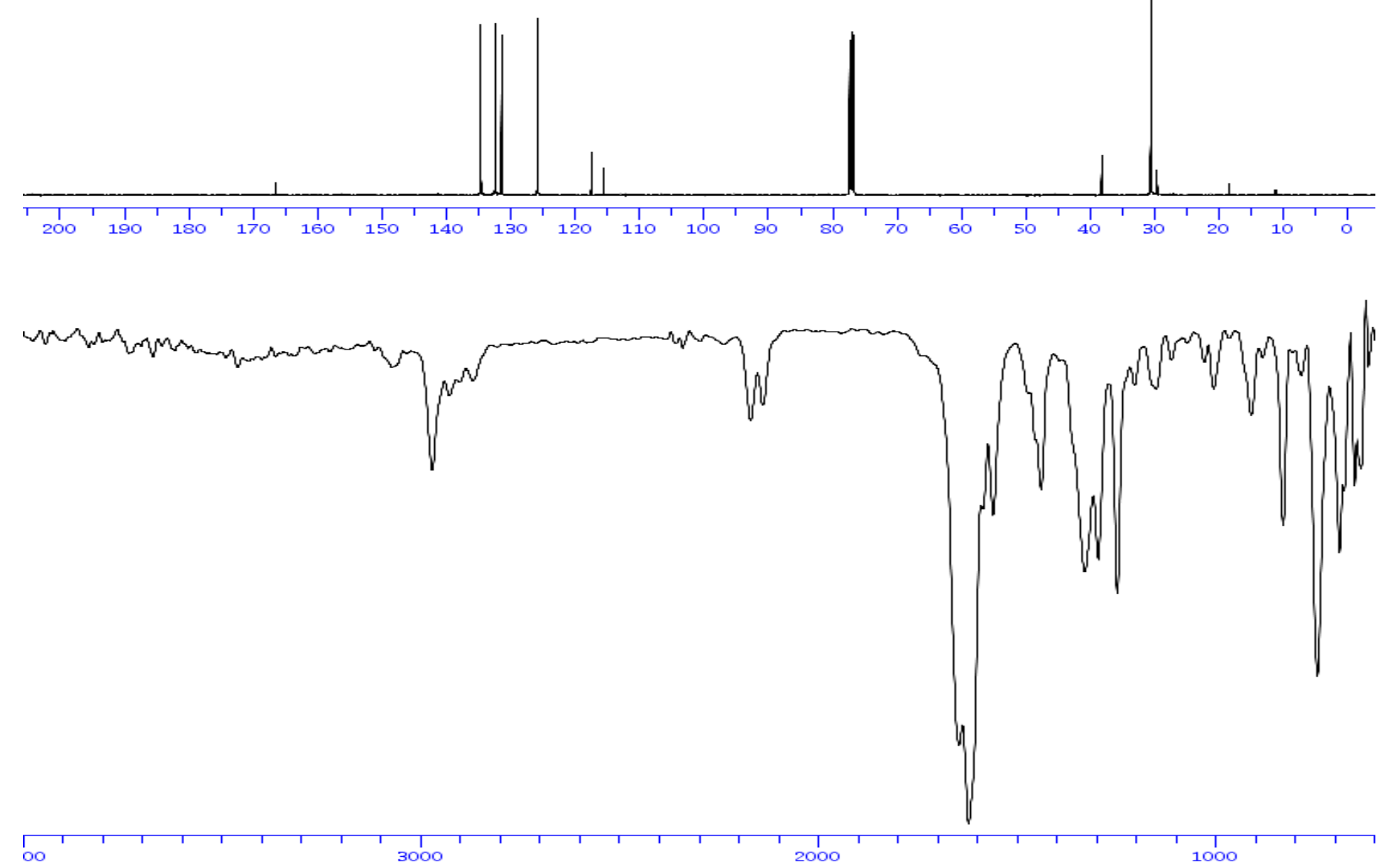

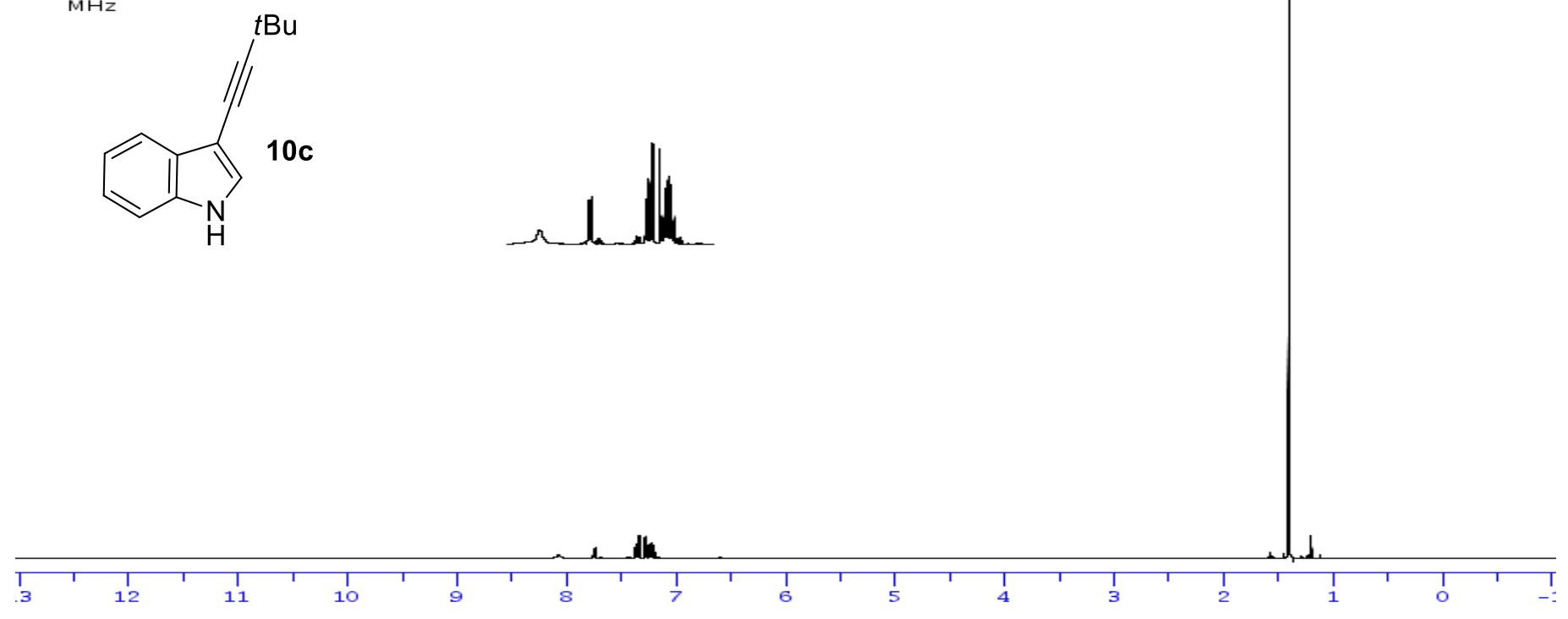

solvent: $<C D C I 3>$
Frequency 100.61

Frequency.
$2769 \mathrm{MHz}$
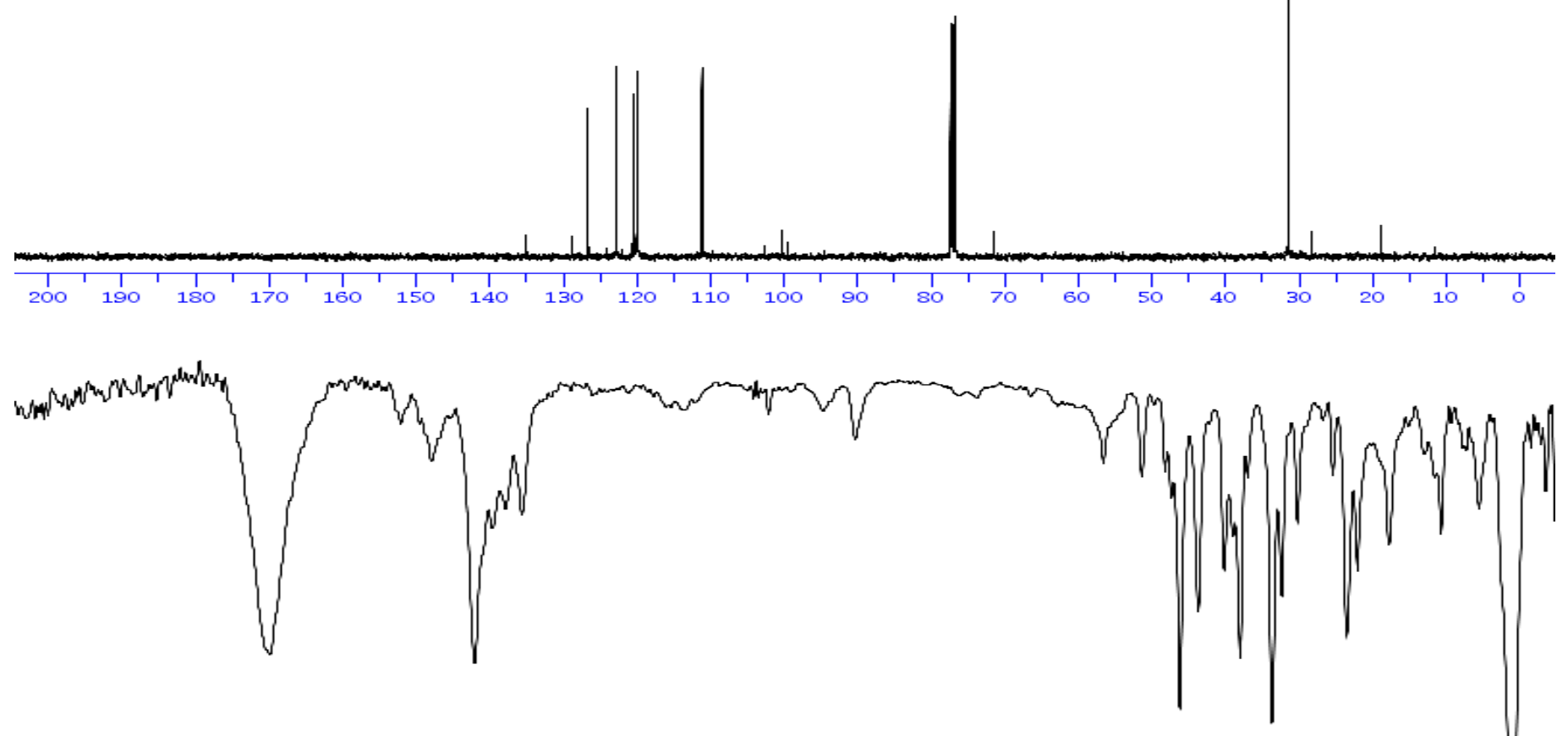
solvent: $<\mathrm{CDCl} 3>$

Frequency. 400.13MH<smiles>Oc1ccc(C#Cc2ccccc2)s1</smiles>

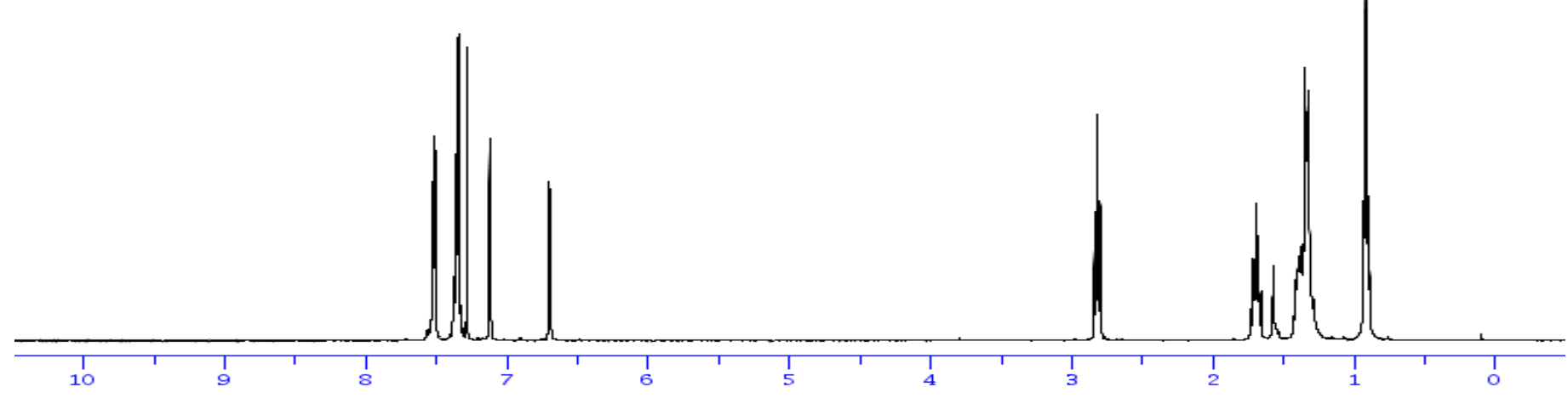

solvent: $<C D C I 3>$
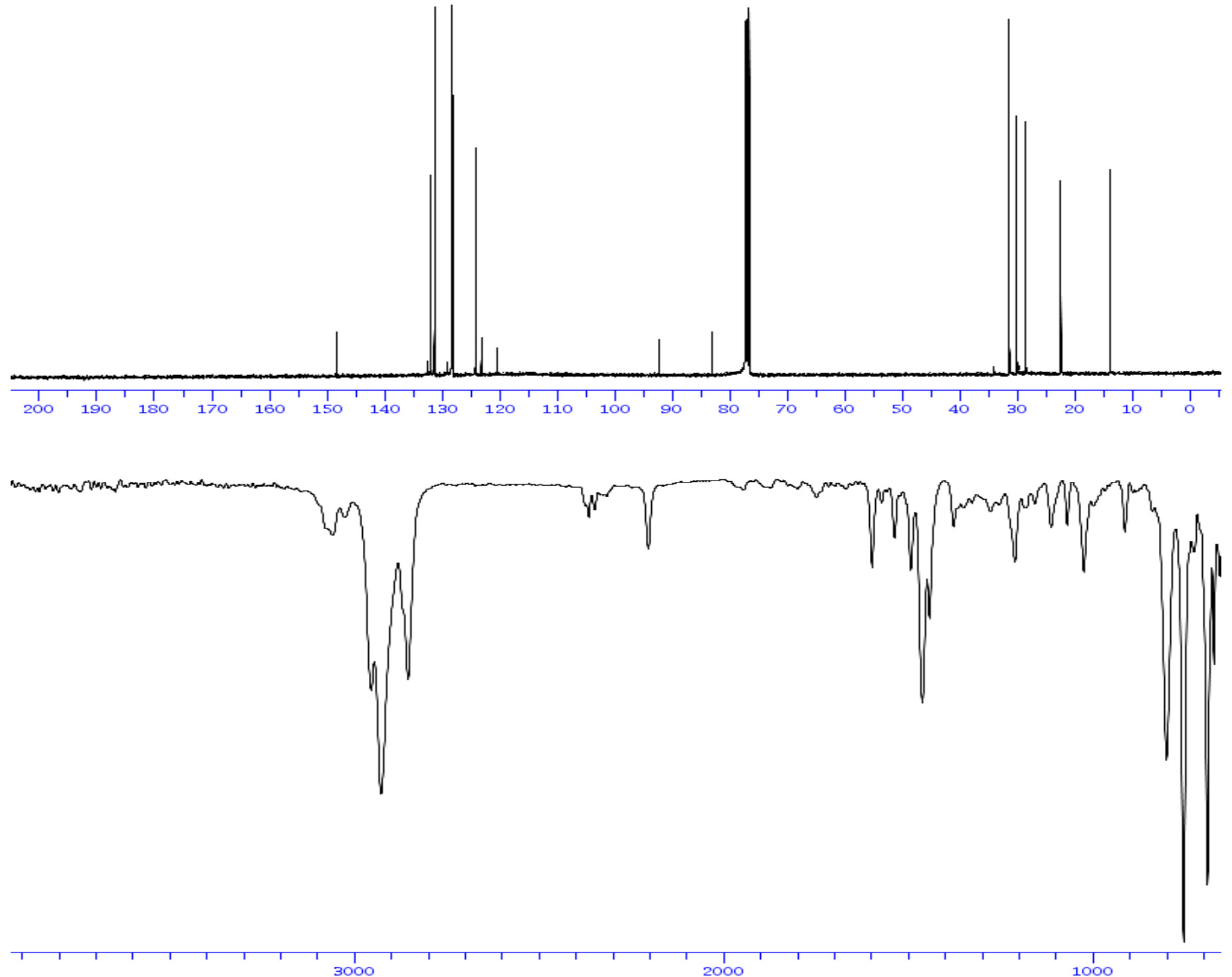
solvent: <CDCI3 >

Frequencr $400.13 \mathrm{MHz}$

Mex

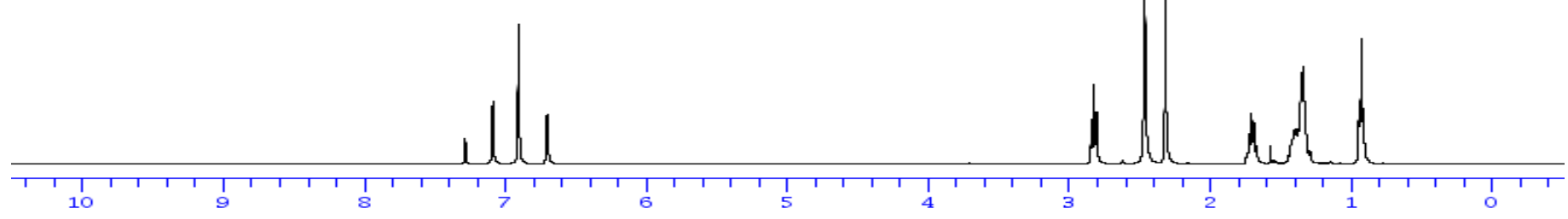

solvent: $<C D C I 3>$
Frequency. $100.612769 \mathrm{MHz}$
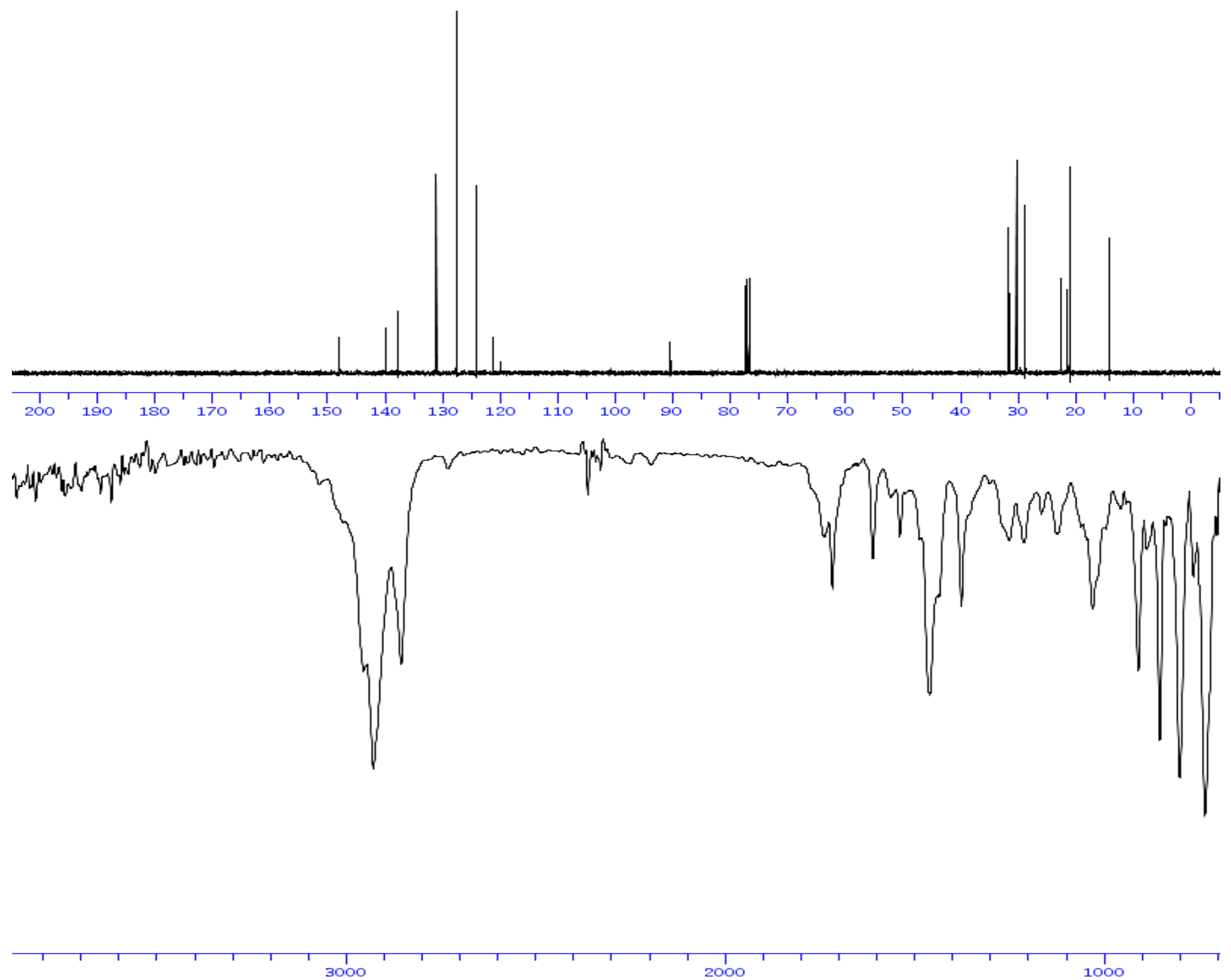


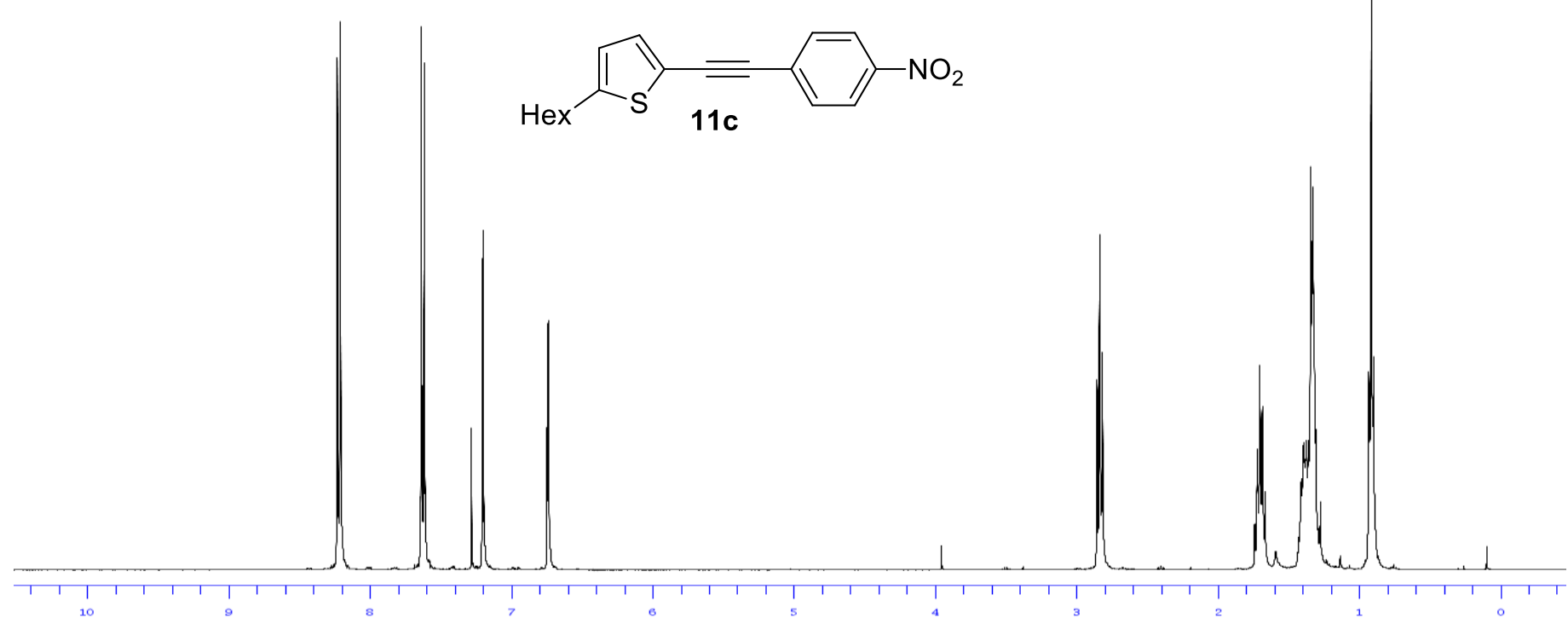

solvent: $<C D C I 3>$
Frequency. $100.612769 \mathrm{MHz}$
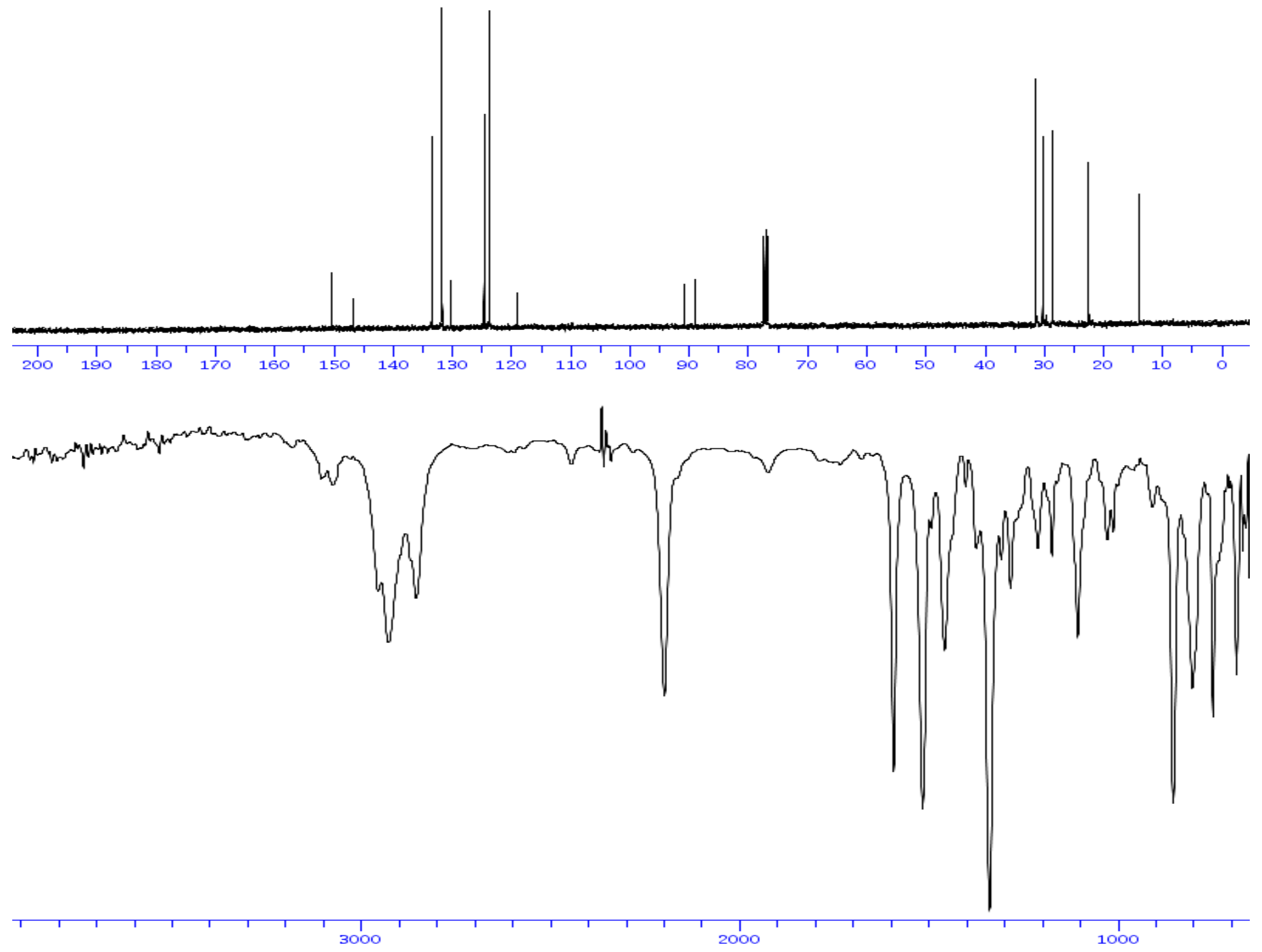
solvent: $<\mathrm{CDCI} 3>$

Frequency $400.13 \mathrm{MH}$

$\overbrace{\mathrm{Sex}}{ }^{t} \mathrm{Bu}$
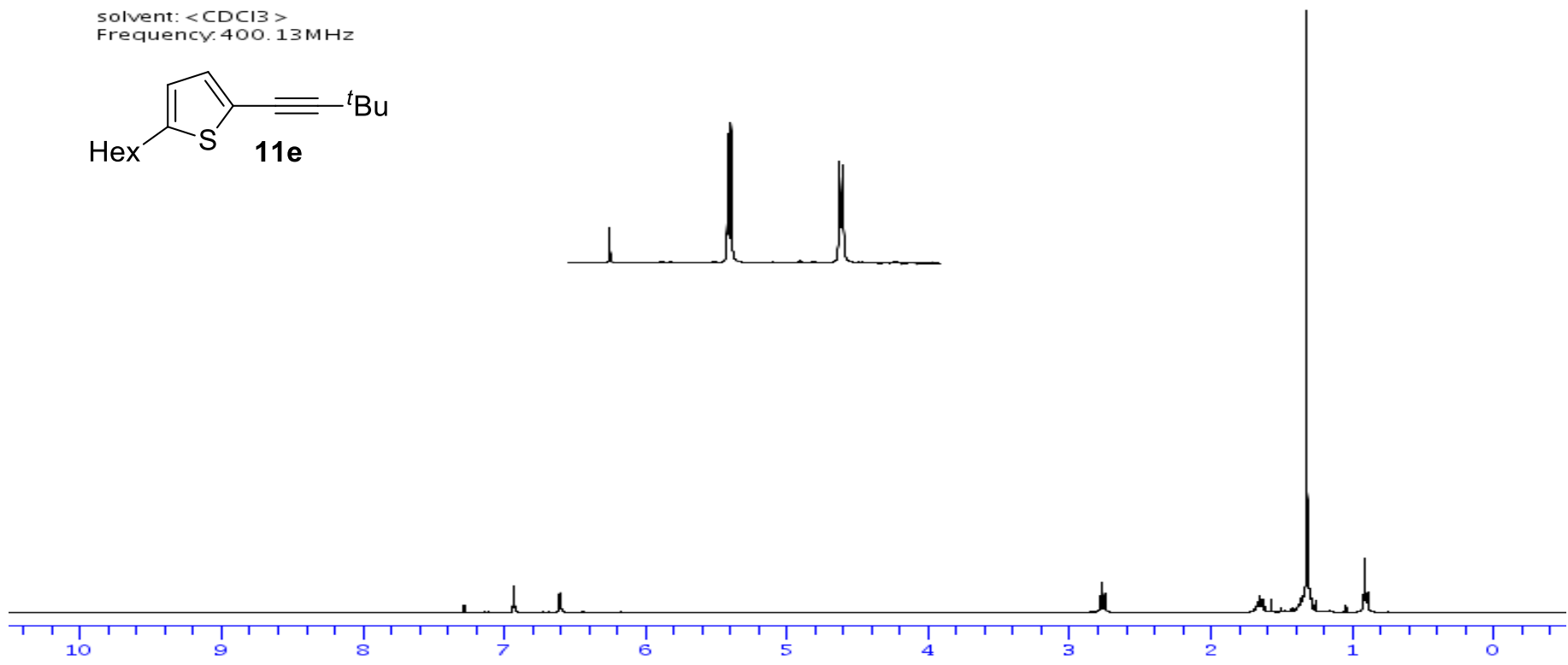

solvent: $<C D C I 3>$
Frequency. $100.612769 \mathrm{MHz}$
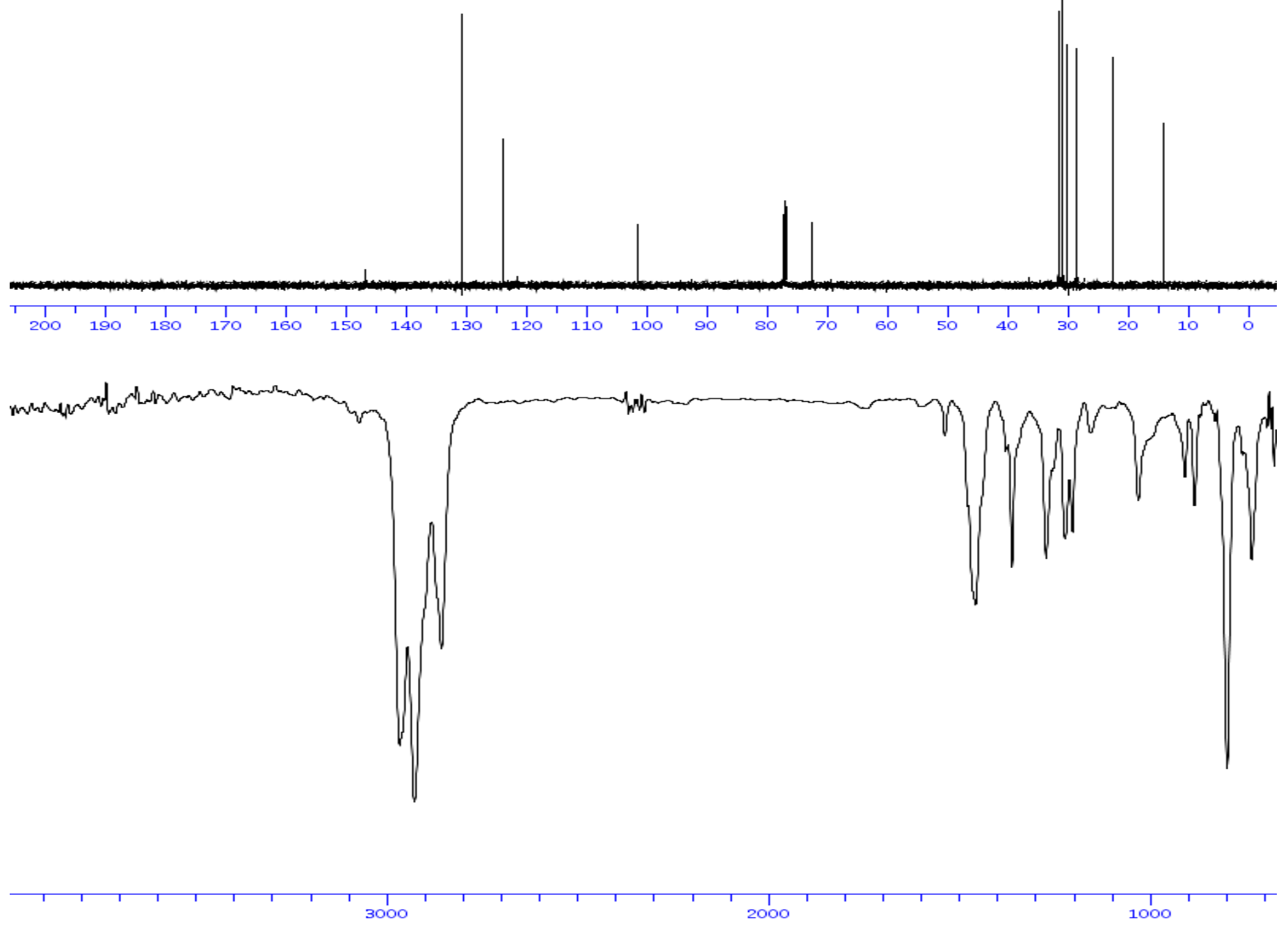
solvent: $<\mathrm{CDCI} 3>$

Frequency $400.13 \mathrm{MHz}$

$\underbrace{\mathrm{OMe}}_{\mathrm{Ph}}$

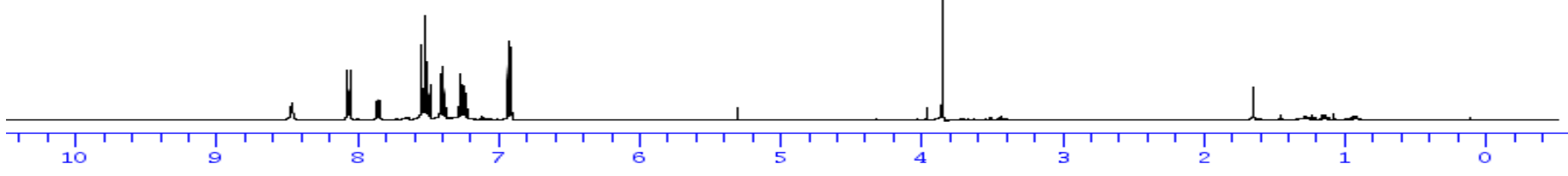

solvent: $<\mathrm{CDCl} 3>$
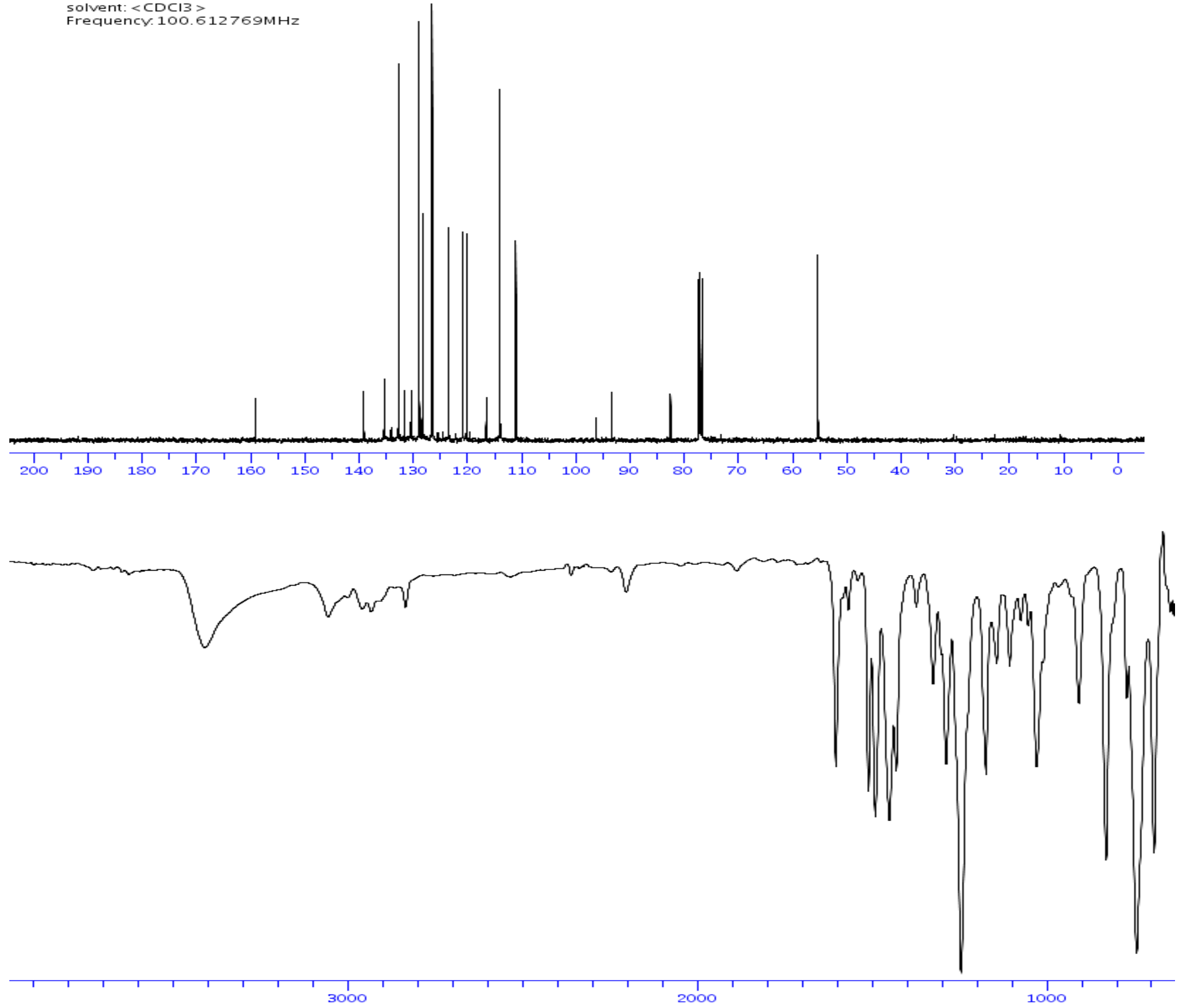
solvent: $\angle \mathrm{CDCl} 3>$

Frequency.400.13 $\mathrm{MHz}$
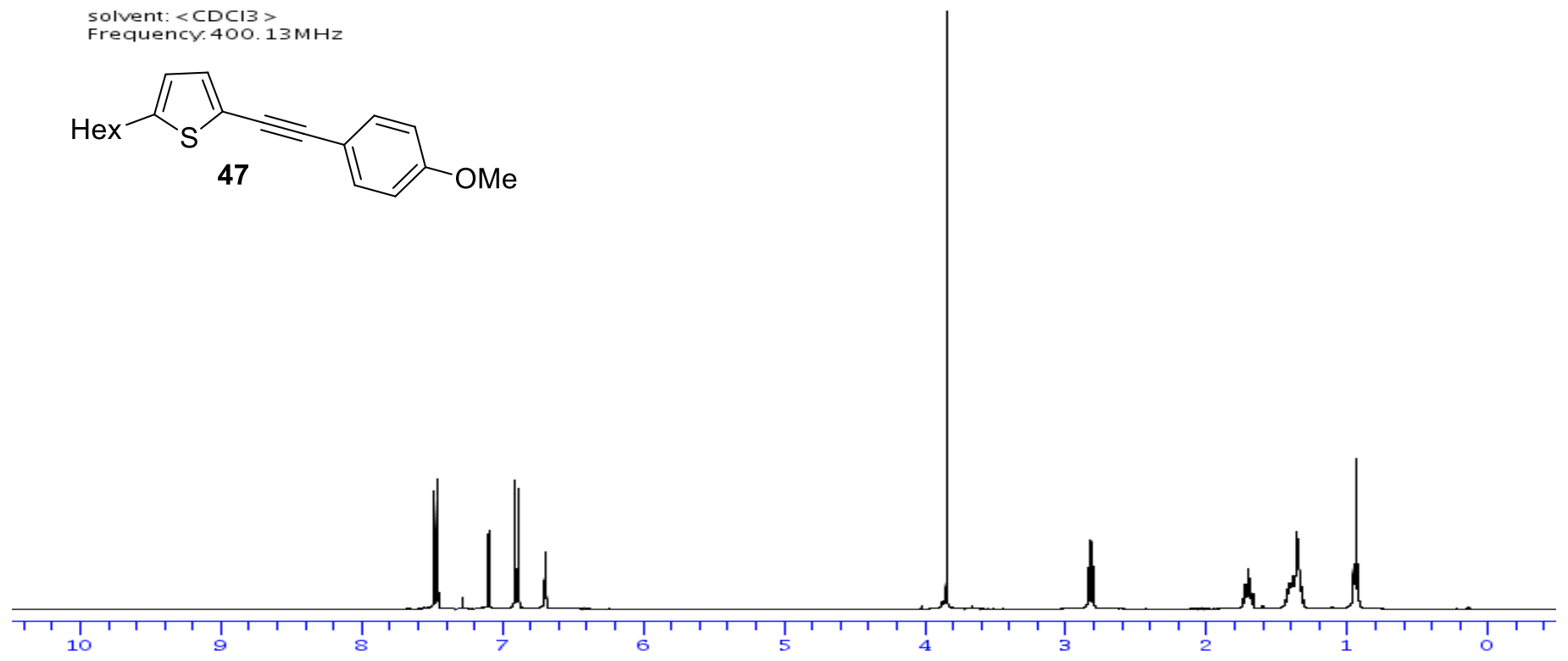

solvent: $\langle\mathrm{CDCl} 3\rangle$

Frequency.100.612769MHz
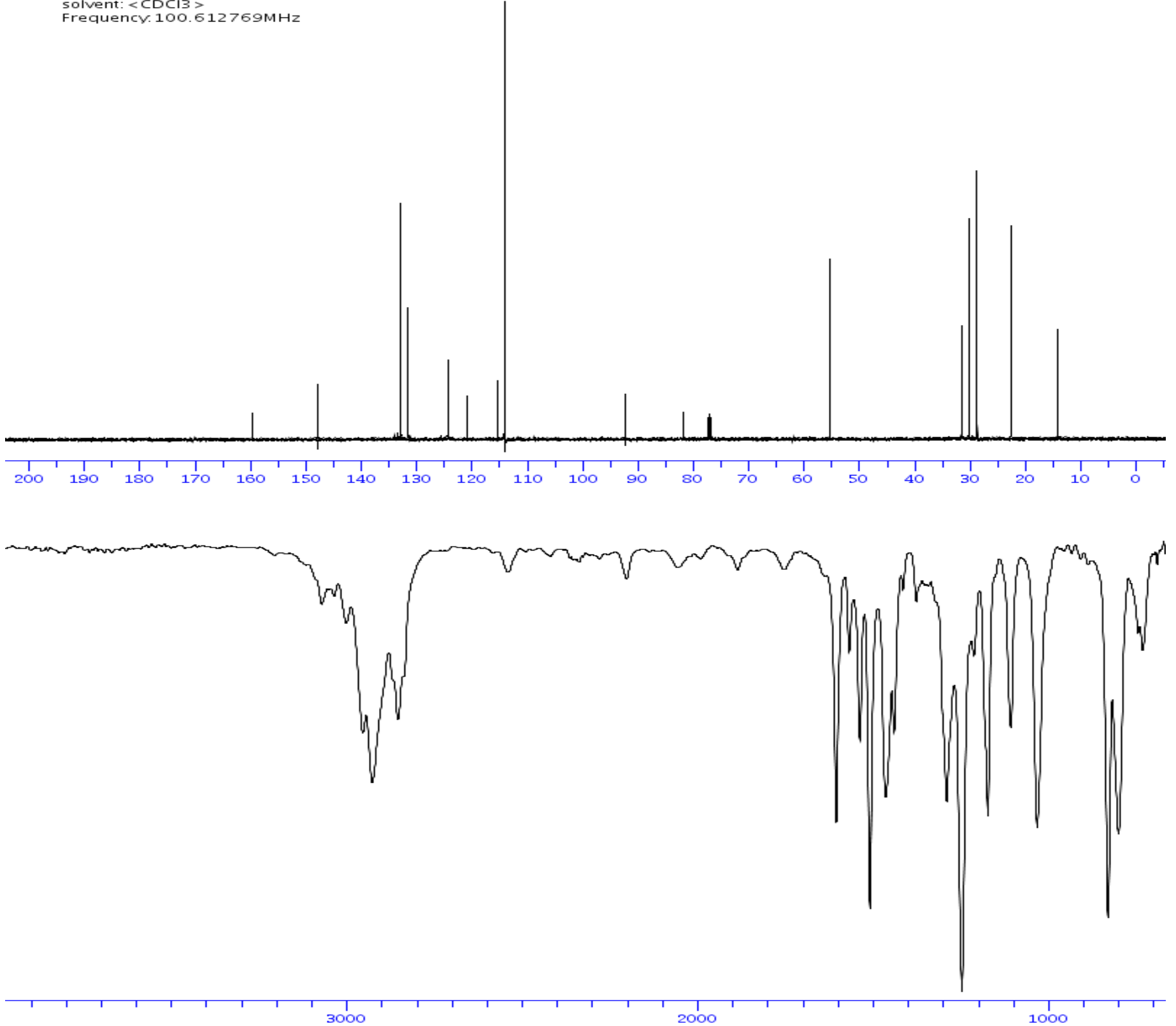
solvent: $<C D C I 3>$

Frequency $400.13 \mathrm{MHz}$<smiles>COc1cc2c(cc1OC)I(C#C[As]C(C)C)OC2=O</smiles>

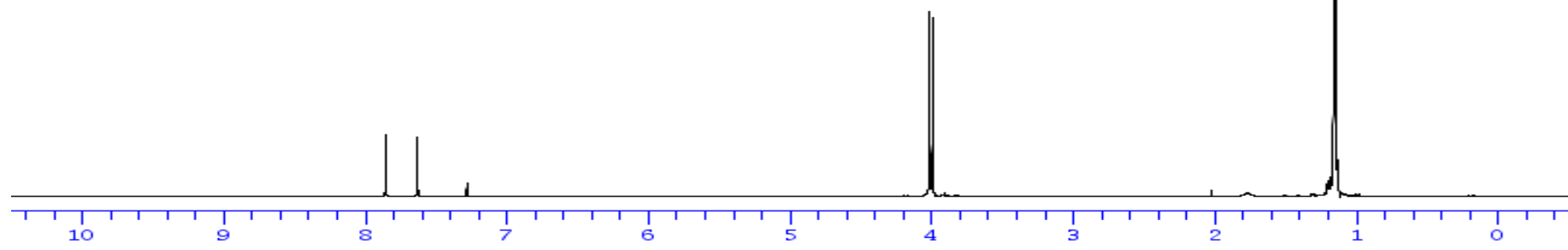

solvent: $\langle\mathrm{CDCI} 3\rangle$

Frequency. $100.612769 \mathrm{MHz}$
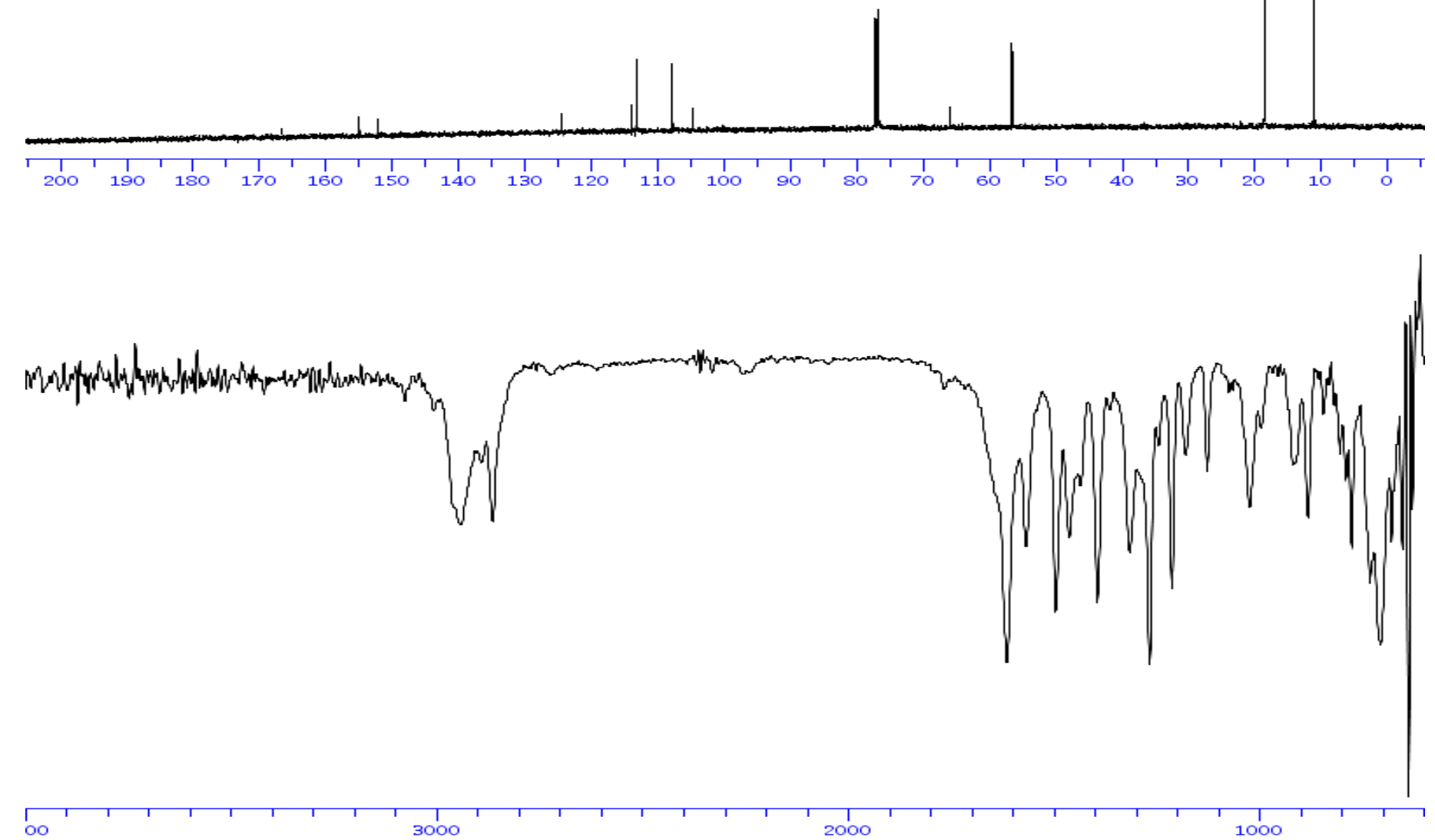
solvent: $\langle\mathrm{CDCI} 3\rangle$

Frequencis

${ }^{i} \mathrm{Pr}_{3} \mathrm{Si}=$

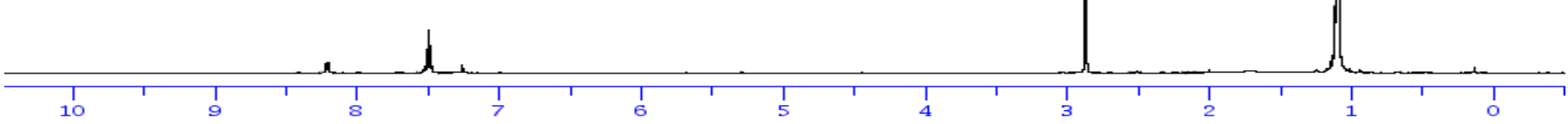

solvent: $\angle \mathrm{CDCI} 3$

Frequency. $100.612769 \mathrm{MHz}$

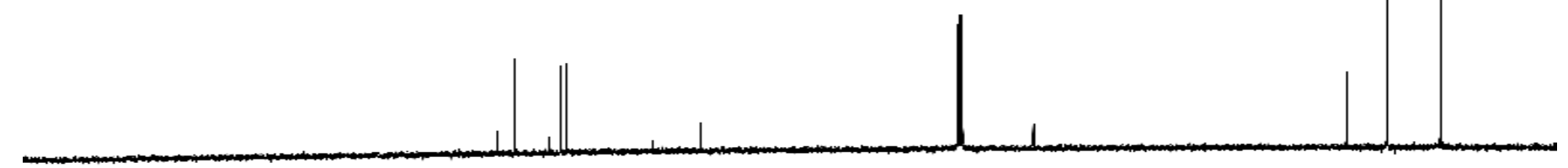

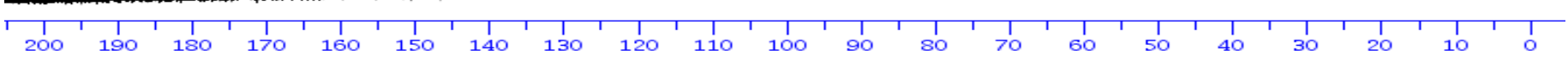


solvent: $\langle\mathrm{CDCI} 3\rangle$

Frequency. 400.13 $\mathrm{MHz}$<smiles>Cc1cccc2c1C(=O)OI2C#C[Se]C(=O)[Mg]</smiles>

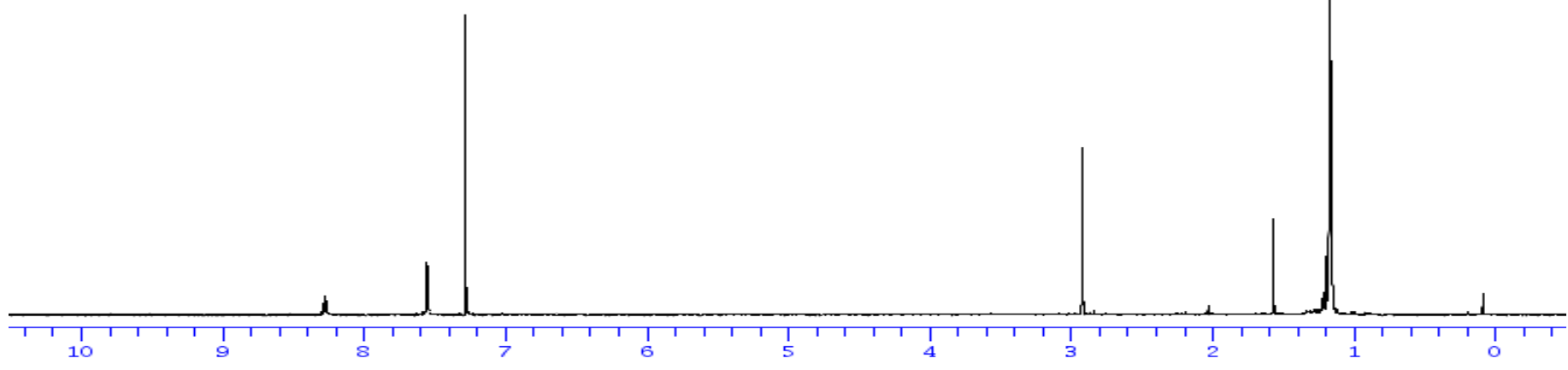

solvent: $<\mathrm{CDCI}$ >

Frequency. $100.612769 \mathrm{MHz}$
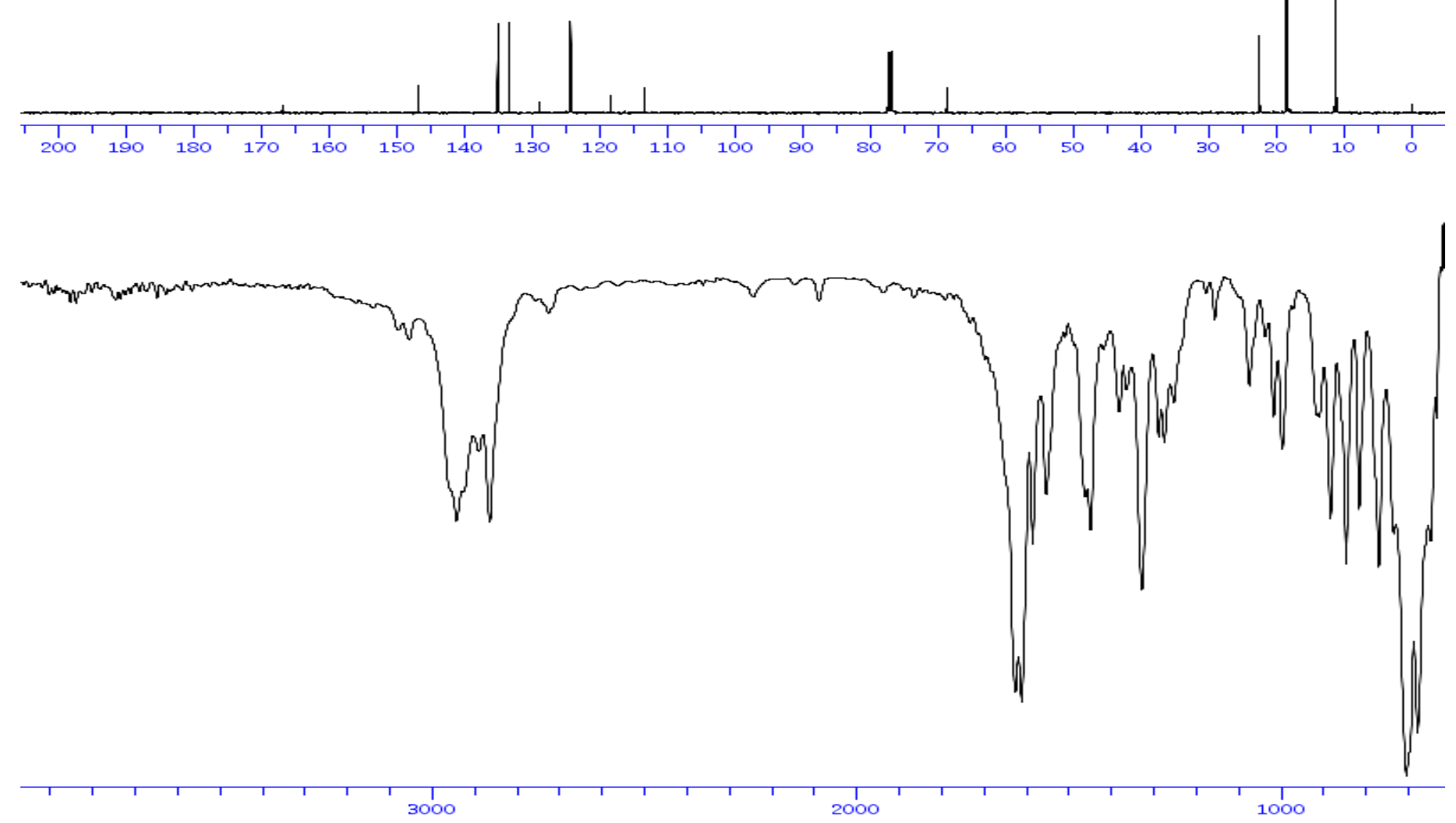
Solvent: <DMSO >

Frequency. 400.13 $\mathrm{MHz}$

$\mathrm{CF}_{\mathrm{CF}_{3}}$

$13 \mathrm{~g}$

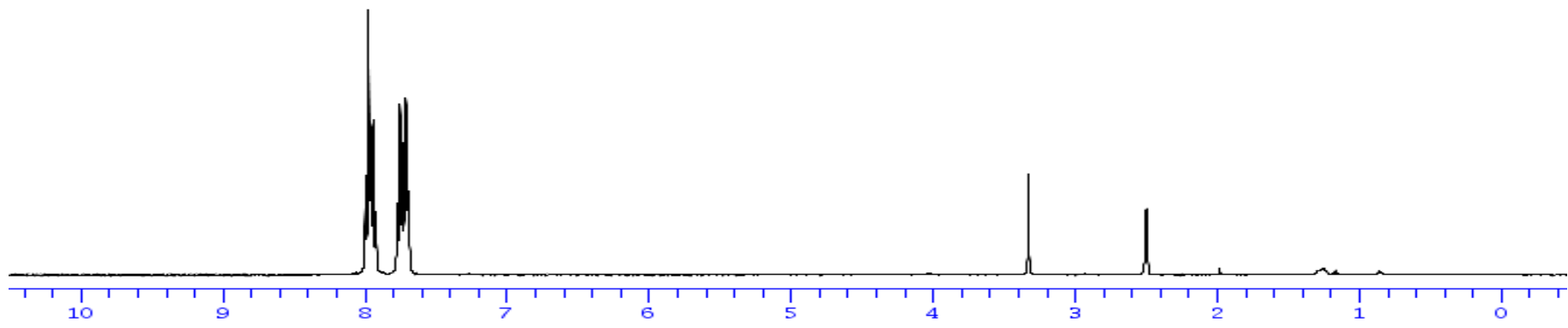

solvent: <DMSO >

quency. $100.612769 \mathrm{MHz}$

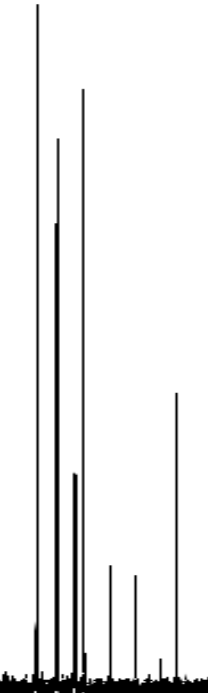

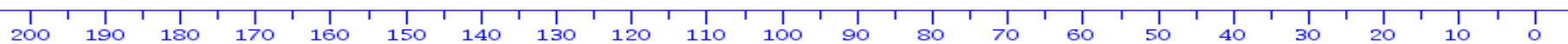

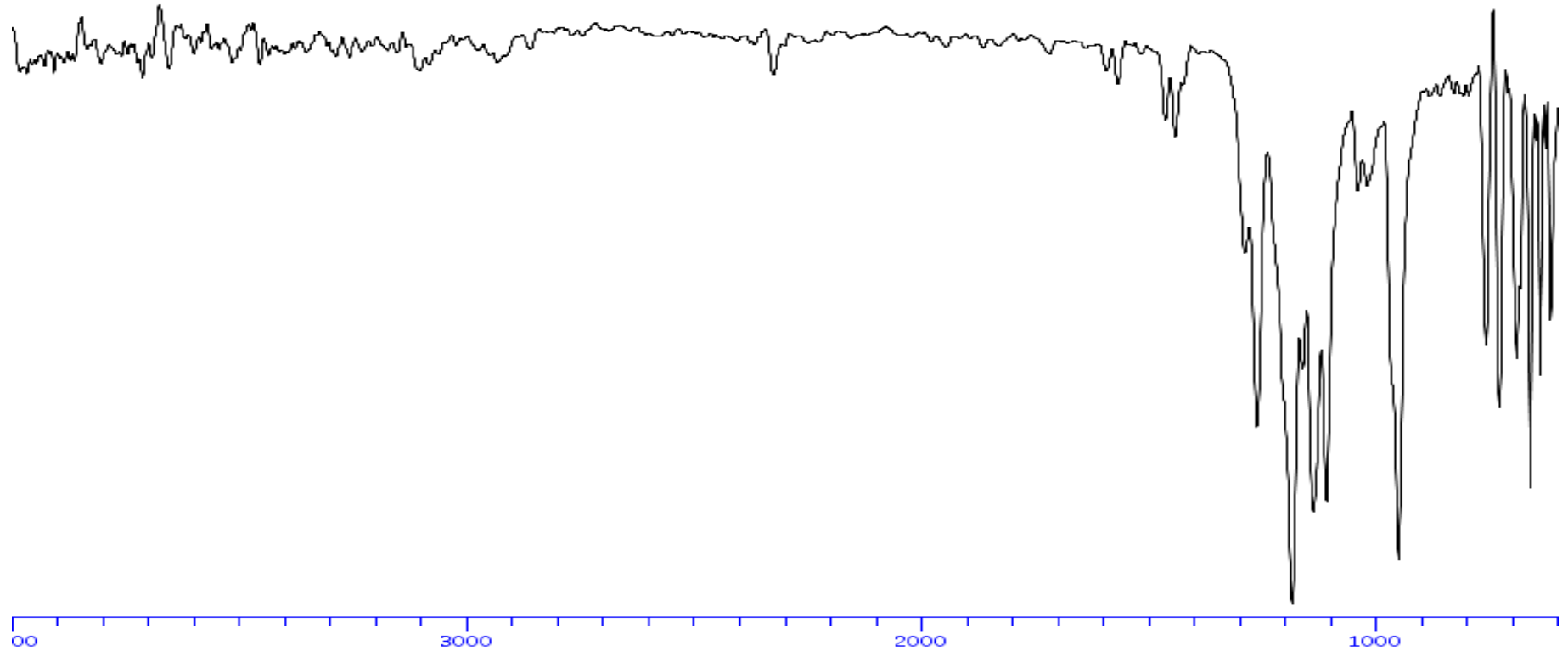


${ }^{i} \mathrm{Pr}_{3} \mathrm{Si}=\mathrm{CFF}_{15 \mathrm{~g}}^{\mathrm{O}}$

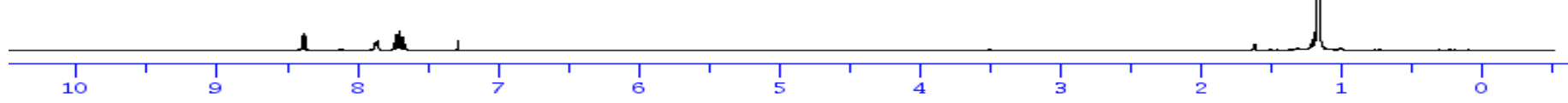

solvent: $<C D C I 3>$
Frequency. $100.612769 \mathrm{MHz}$
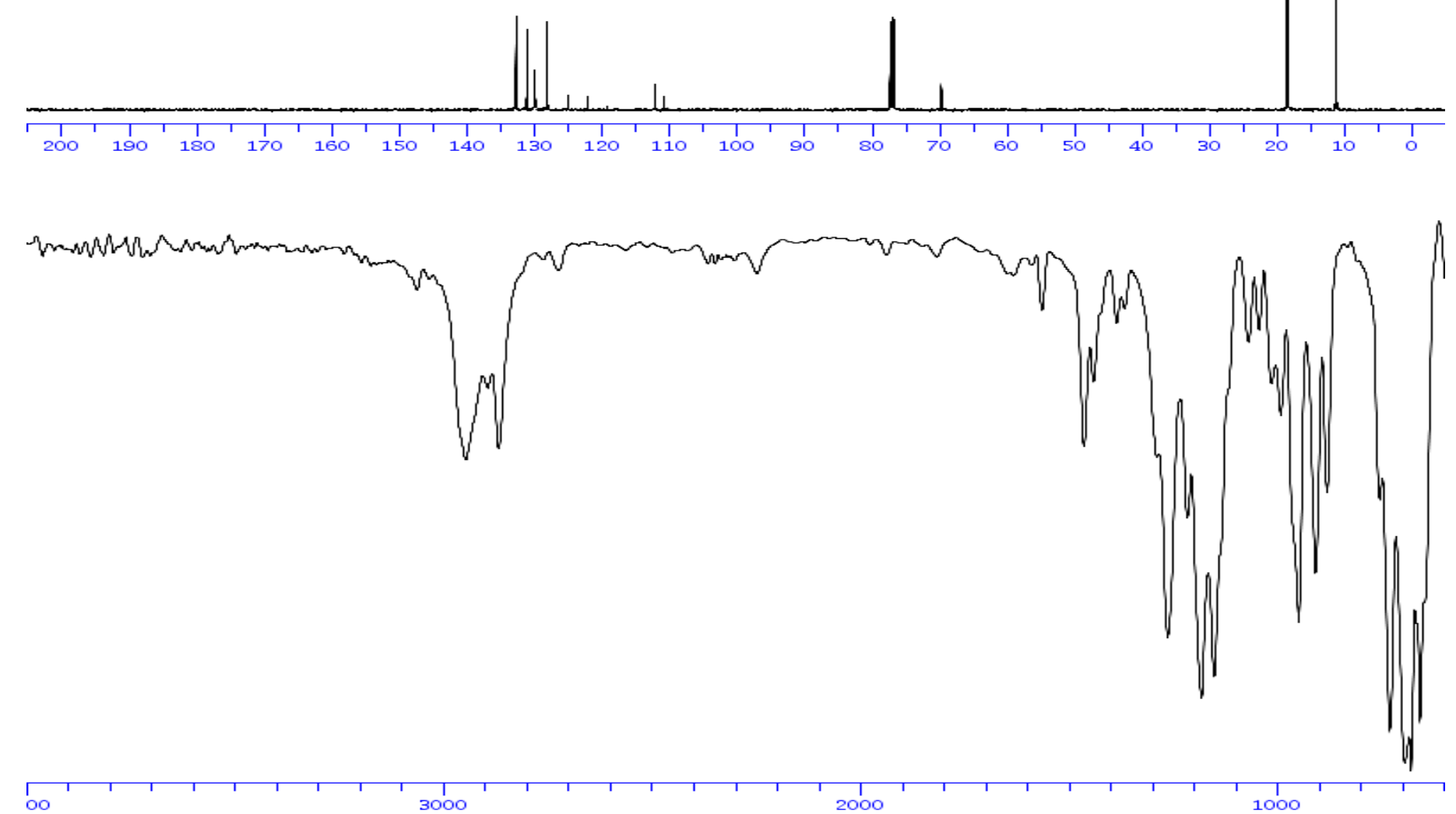
Solvent: $\angle$ DMSO $>$

Frequency. $400.13 \mathrm{MHz}$

$\overbrace{\mathrm{Me}}^{\mathrm{C}} \mathrm{Me}$

16

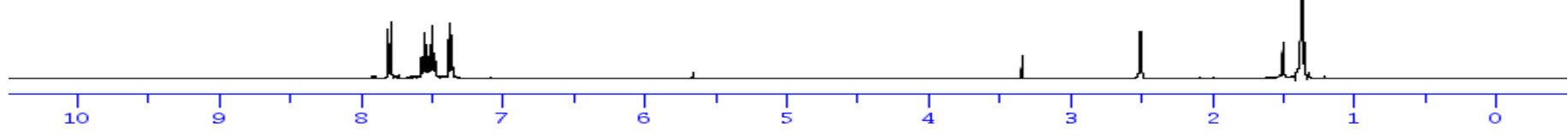

solvent: DMSO

Frequency. $100.612769 \mathrm{MHz}$

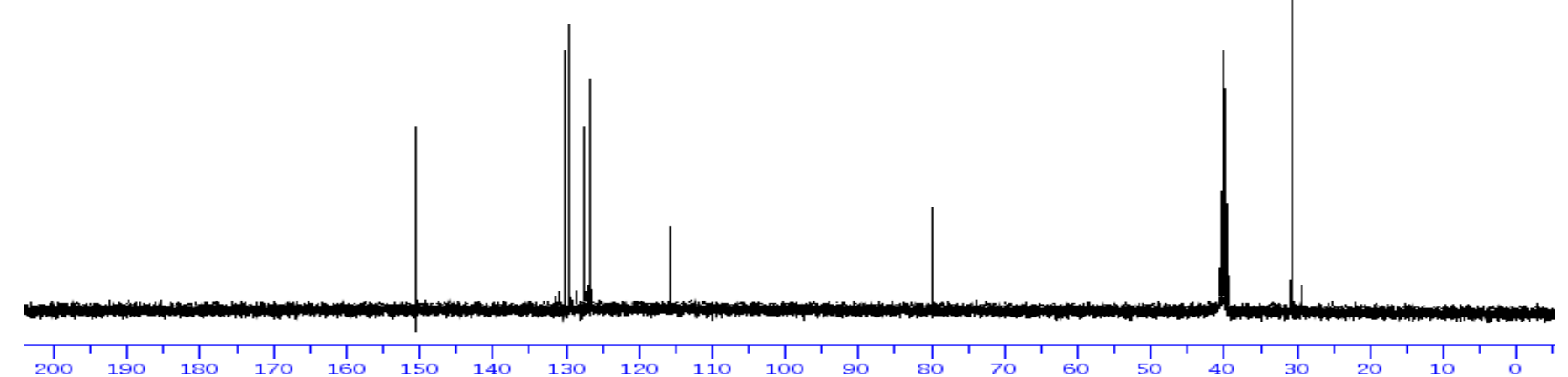

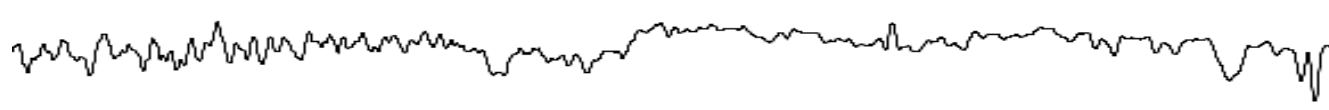

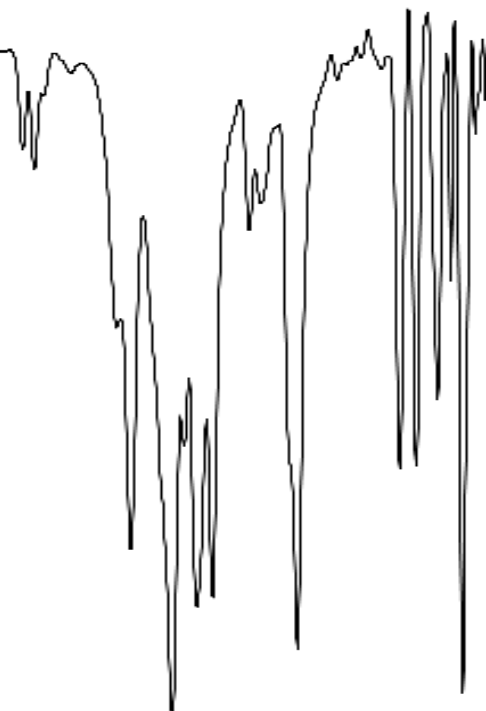

oo 1000 
solvent: $\langle\mathrm{CDCl} 3\rangle$

solvent: $\mathrm{CDCl}>13 \mathrm{MHz}$

${ }_{\mathrm{Pr}_{3} \mathrm{Si}}=\mathrm{C}_{\mathrm{Me}}^{\mathrm{O}}$
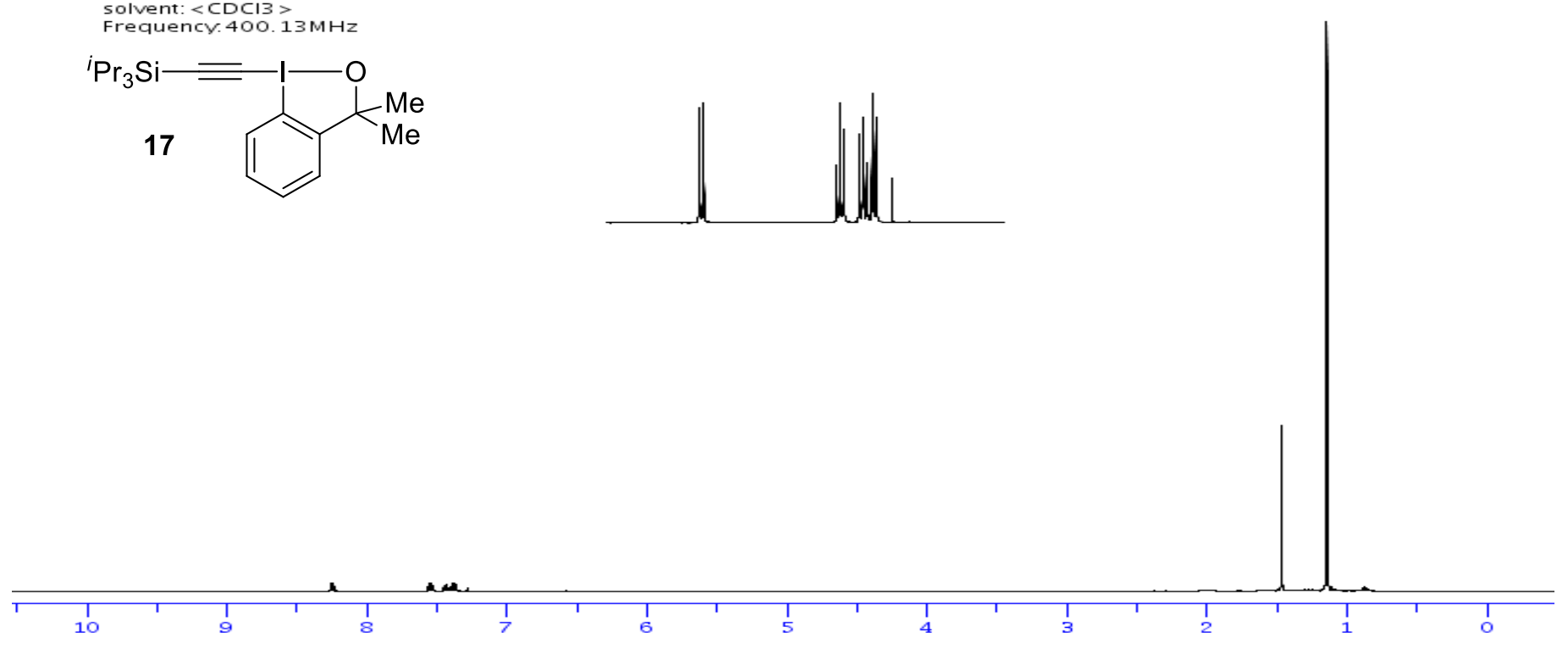

Solvent: $<\mathrm{CDCI} 3>$
Frequency. $100.612769 \mathrm{MHz}$
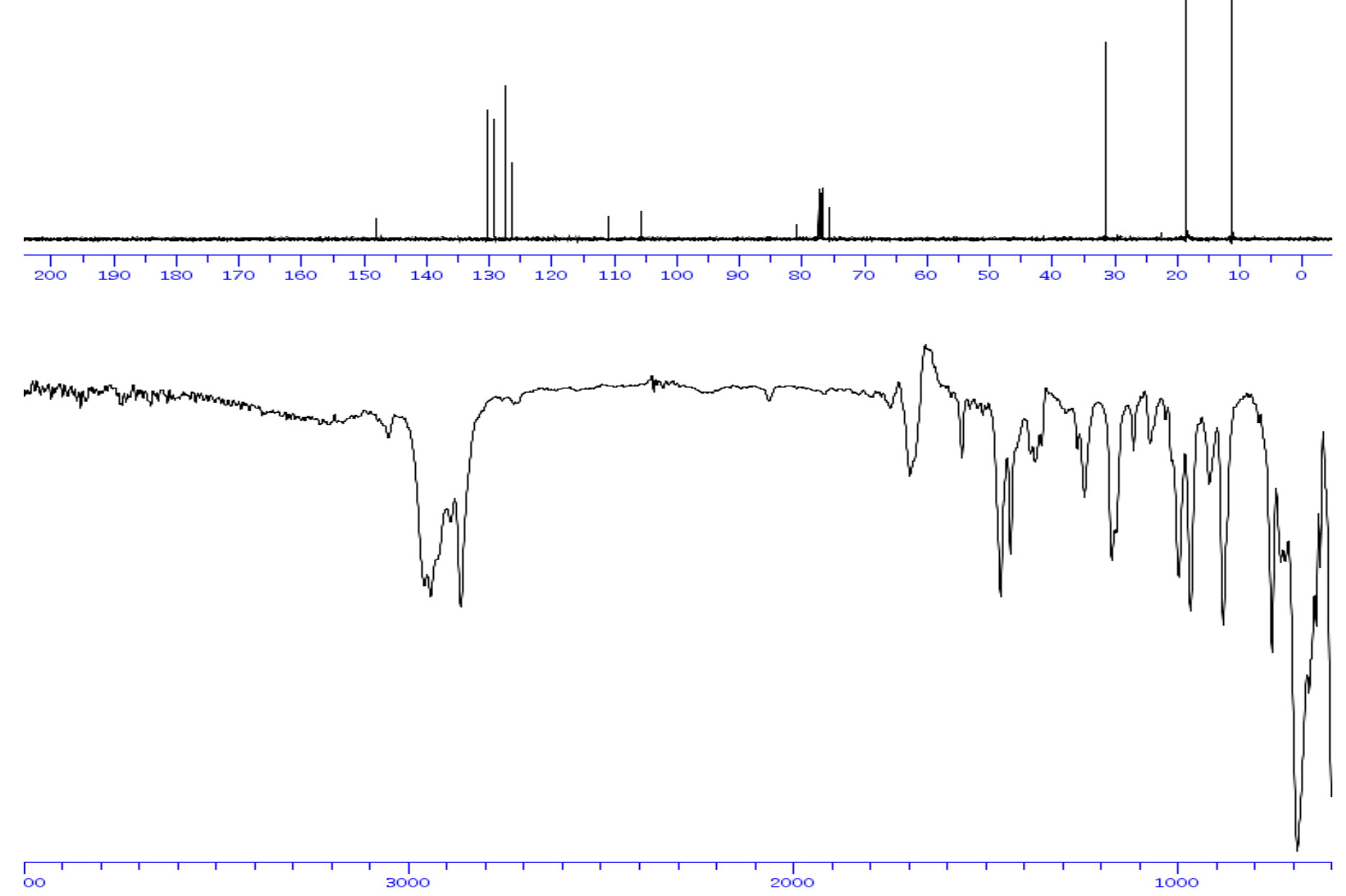
solvent: $<C D C I 3>$
Frequency. $400.13 \mathrm{MHz}$

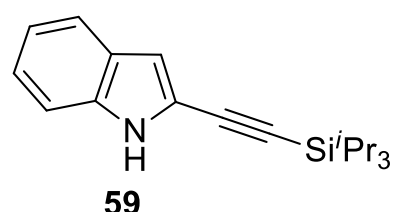

59
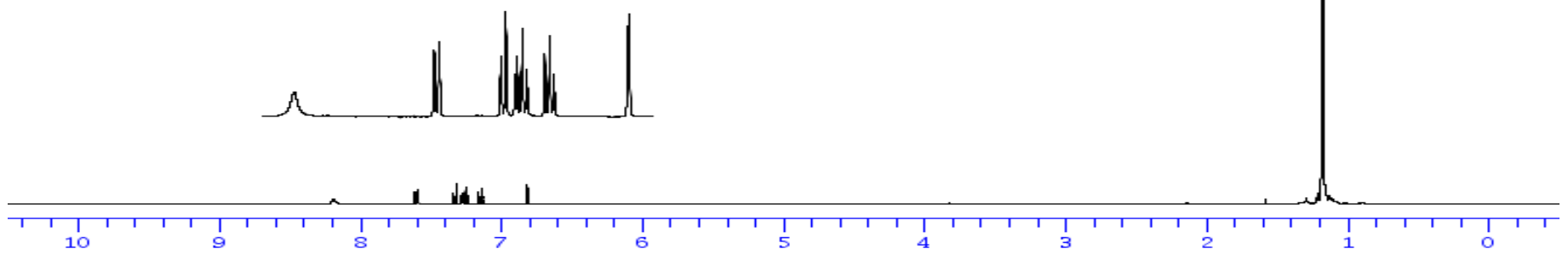

Solvent: $<C D C I 3>$
Frequency. $100.612769 \mathrm{MHz}$
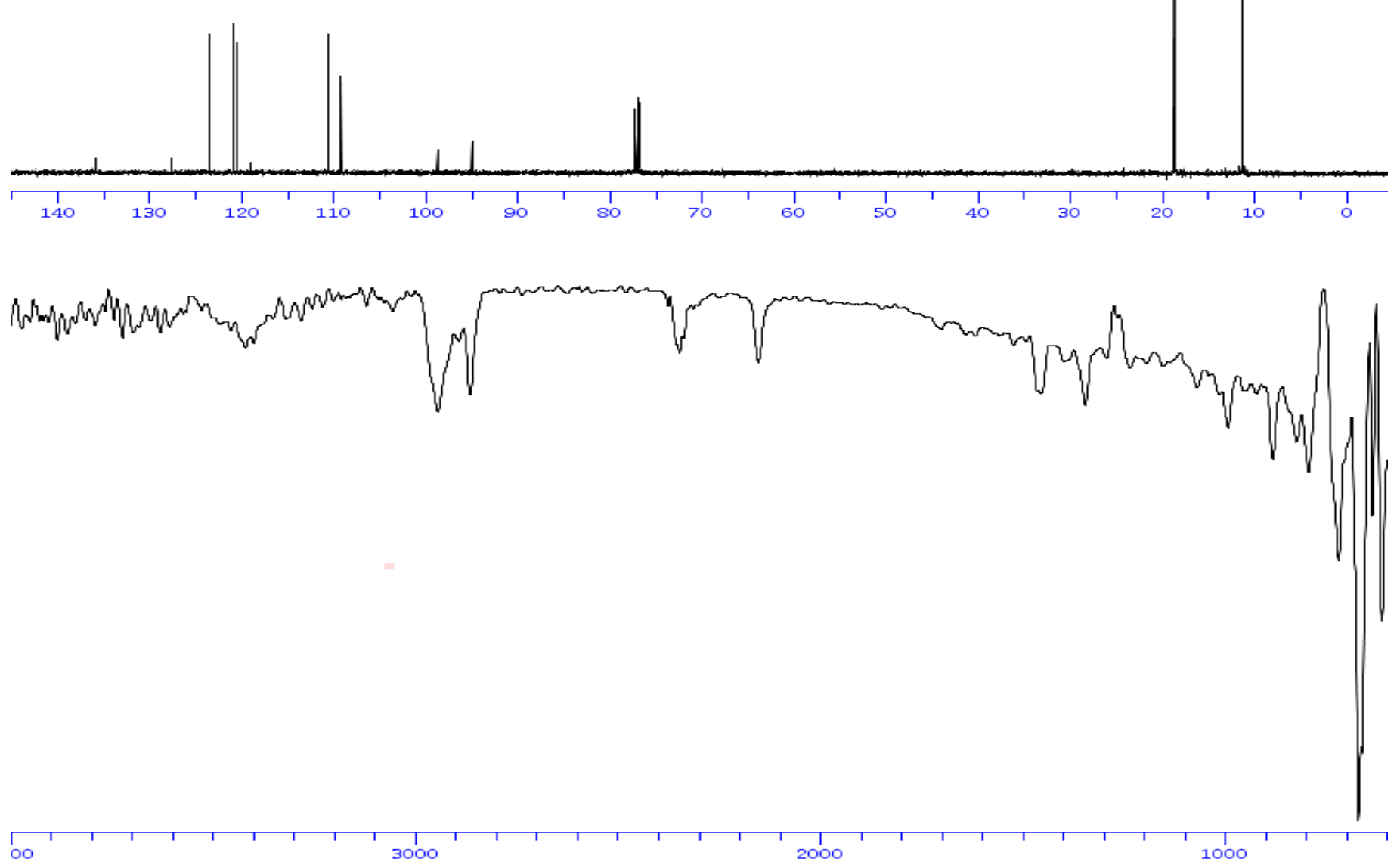

67 